\title{
ON THE GENETIC MECHANISMS OF NUTRIENT-DEPENDENT LIFESPAN AND REPRODUCTION
}

Jelle Zandveld 


\section{Thesis committee}

\section{Promotor}

Prof. B.J. Zwaan

Professor of Genetics

Wageningen University \& Research

\section{Co-promotor}

Dr A.J.M. Debets

Associate professor, Laboratory of Genetics

Wageningen University \& Research

\section{Other members}

Prof. Dr A.H. Kerstens, Wageningen University \& Research,

Prof. Dr P. Slagboom, Leiden University Medical Centre, the Netherlands

Dr M. Simons, University of Sheffield, United Kingdom

Prof. Dr B. Wertheim, Groningen University, the Netherlands

This research was conducted under the auspices of the C.T. de Wit Graduate School for Production Ecology and Resource Conservation 


\section{ON THE GENETIC MECHANISMS OF NUTRIENT-DEPENDENT LIFESPAN AND REPRODUCTION}

Jelle Zandveld

Thesis

submitted in fulfilment of the requirements for the degree of doctor at Wageningen University

by the authority of the Rector Magnificus

Prof. Dr A.P.J. Mol, in the presence of the

Thesis Committee appointed by the Academic Board to be defended in public

on Wednesday 11 October 2017 at 4 p.m. in the Aula. 
Jelle Zandveld

On the genetic mechanisms of nutrient-dependent lifespan and reproduction 212 pages.

$\mathrm{PhD}$ thesis Wageningen University, Wageningen, the Netherlands (2017) With references and summary in English

ISBN 978-94-6343-686-1

DOI http://dx.doi.org/10.18174/422511 


\section{TABLE OF CONTENTS}

\section{CHAPTER I}

General introduction (p.8)

\section{CHAPTER II}

Both overlapping and independent mechanisms determine how diet and insulin-ligand knockouts extend lifespan of Drosophila melanogaster (p.24)

\section{CHAPTER III}

Gene expression responses upon knockout of insulin-like peptides in Drosophila melanogaster (p.42)

\section{CHAPTER IV}

Natural genetic variation in the response to nutrition in the fungus Podospora anserina reveals that lifespan and reproduction can be uncoupled (p.62)

\section{CHAPTER V}

Experimental evolution of nutrient-dependent reproduction in Drosophila melanogaster (p.80)

\section{CHAPTER VI}

Pervasive gene expression responses to a fluctuating diet in Drosophila melanogaster (p.110)

\section{CHAPTER VII}

Lifespan and reproduction relationships explain consistent inconsistencies in whole-genome transcription responses to diet in Drosophila melanogaster (p.144)

\section{CHAPTER VIII}

Synthesis (p. 174)

\section{REFERENCES (p.188)}

SUMMARY (p.200)

ACKNOWLEDGEMENTS (p.202)

CURRICULUM VITAE (p. 206)

EDUCATION STATEMENT (p. 210) 


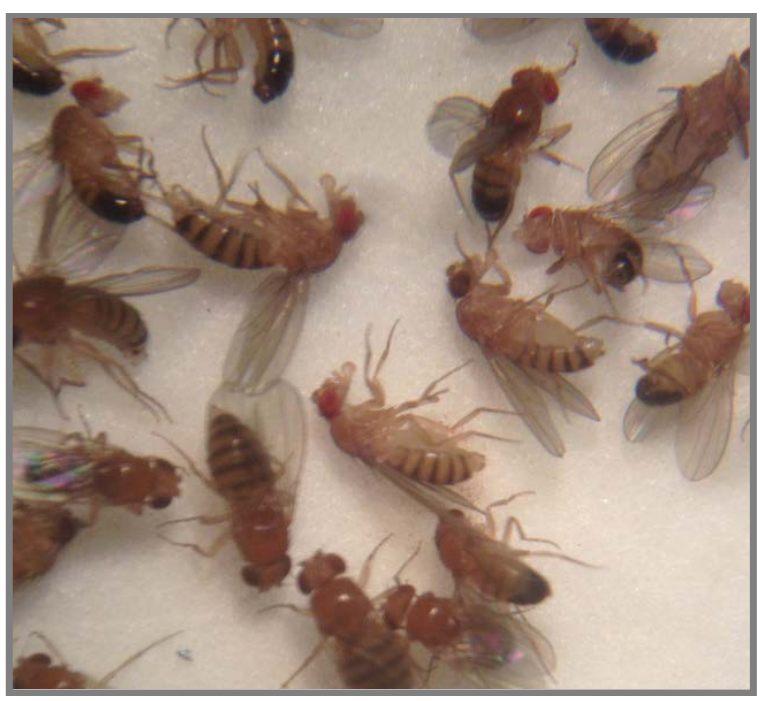




\section{De ontdekking}

Als je goed om je heen kijkt

Zie je dat alles gekleurd is

K. Schippers 


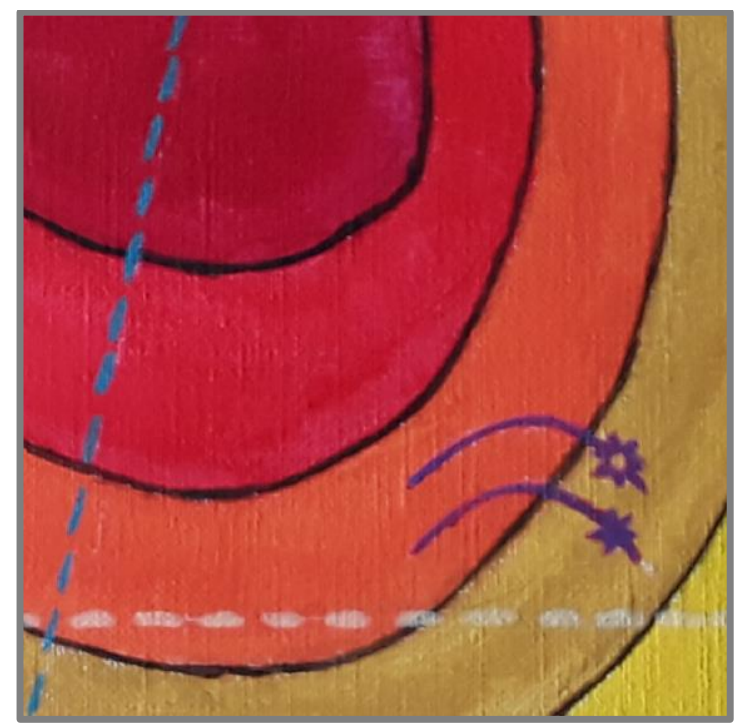




\section{CHAPTER I}

\section{GENERAL INTRODUCTION}

On the genetic mechanisms of nutrient-dependent lifespan and reproduction 


\section{CHAPTER 1: GENERAL INTRODUCTION}

On the genetic mechanisms of nutrient-dependent lifespan and reproduction

In the last 50 years, human life expectancy has risen by more than 20 years in the Netherlands (CBS Statline, 2017), and will likely further increase due to continuously improving health care, medication and hygiene. Inevitably, this life expectancy increase is accompanied with an increase of age-related diseases and raises the call for a better understanding of age-related morbidity and mortality, i.e. ageing. Similar to humans, other organisms also show signs of ageing upon removal of environmental hazards (Jones et al. 2014). Therefore, studying the ageing process of organisms in the laboratory may potentially give insights into the generalities of the ageing process between organisms. An environmental intervention that increases lifespan and that is often studied in the laboratory is dietary restriction, generally defined as a reduction of food intake without malnutrition (Weindruch and Walford, 1988). In many organisms dietary restriction extends lifespan, but the exact mechanisms how this intervention extends lifespan, are not yet completely understood. In this thesis I focused on genetic effects on the diet responses in two laboratory models, Drosophila melanogaster and Podospora anserina, in order to uncover potential mechanisms that play a role in mediating such responses.

\section{AGEING}

Ageing is defined as the progressive loss of function accompanied by decreasing fertility and increasing mortality with advancing age (Kirkwood \& Austad 2000). Evolutionary theory has provided an answer to the question, why does ageing occur? Because ageing is highly disadvantageous for the individual, one would expect that natural selection should remove alleles that increase ageing. However, in an environment of high extrinsic mortality, i.e. through accidents, infections, disease and predation, lifespan is short (Figure 1a) and selection pressure will be generally weaker on traits expressed only late in life (i.e. the selection shadow, Figure 1b). Therefore, such reduced selection pressure for any allelic effect late in life, facilitated ageing to evolve. Namely, when protected against extrinsic hazards, individuals will live longer, but eventually suffer and die from the late acting detrimental traits accumulated during evolution. This theory is formulated by Medawar (1952) in his theory of mutation accumulation (MA). Modern society is an example of a protected population, and because also in human evolution there were likely extrinsic hazards such as disease and accidents, this theory also applies to humans and can explain why humans age. Comparable to modern society humans, laboratory animals are also removed from many extrinsic hazards and therefore express age-related decline in function (Figure 1a), i.e. ageing.

George Williams published an extension of the MA hypothesis by proposing the Antagonistic Pleiotropy (AP) theory of ageing. Similar to the MA theory, also Williams believed that (late life) 
damaging mutations cause ageing, but proposed that these mutations may actually be favoured by natural selection because of an advantage early in life (Figure 1c). When the sum of the early and late-life effects will eventually positively contribute to the organism's fitness, this allele will be selected for (Williams 1957). The third, mutually inclusive theory of ageing is the disposable soma theory (DS). This theory was proposed by Thomas Kirkwood who assumed that there is a limited amount of resources to be allocated between somatic maintenance and other processes and traits related to fitness, such as reproduction. In environments with increased hazards, much investment of the scarce resources to maintenance and repair will be maladaptive when it involves costs for reproductive performance (Figure 1d). So, due to an increased risk of mortality through disease, accidents, or predation in the wild, most organisms would evolve a limited investment to maintenance and repair, including humans (Kirkwood 1977). Species differences in lifespan could therefore be explained by their different life-history strategy, i.e. the series of events related to fitness that occur from birth to death (Stearns 1992; Roff 2001).

a

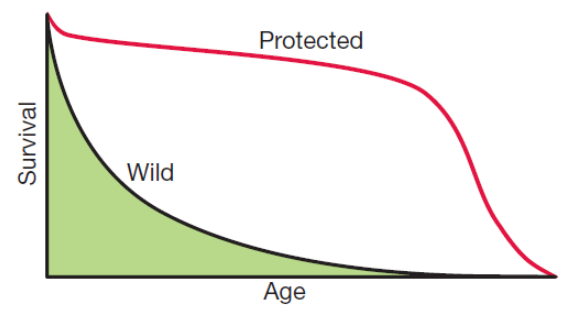

。

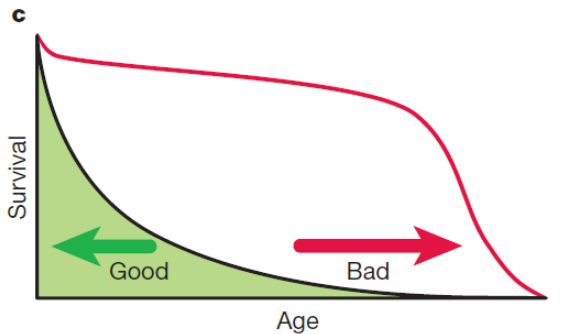

b

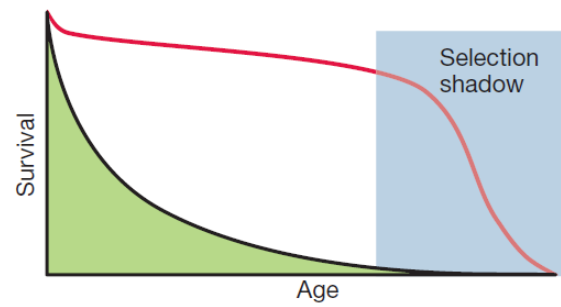

d

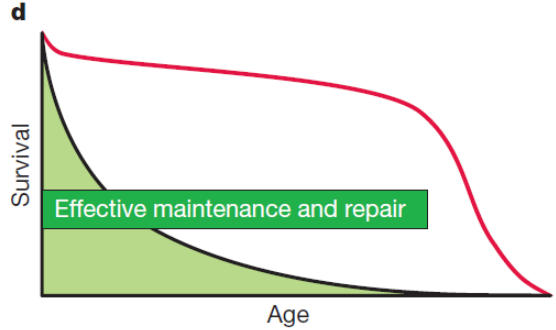

Figure 1: Evolutionary theories of ageing. (a) In the wild, extrinsic mortality occurs to an extent that senescence-associated mortality is rare, dismissing explanations that genes specifically for ageing have evolved. (b) The 'selection shadow' at older ages may permit an accumulation of late-acting deleterious mutations (mutation-accumulation theory). (c) Pleiotropic genes that benefit organisms early in life will be favoured by selection even if they have bad effects at later ages (pleiotropy theory). (d) Selection pressure to invest metabolic resources in somatic maintenance and repair is limited; all that is required is to keep the organism in sound condition for as long as it might survive in the wild (disposable-soma theory). Figure and caption from: Kirkwood and Austad (2000) 


\section{EVOLUTION OF A LIFESPAN REPRODUCTION TRADE-OFF}

According to these theories of ageing, a release of strong extrinsic mortality should result in the evolution of lifespan extension, an effect that has been tested in the laboratory. And indeed, laboratory populations with a low adult mortality (Stearns et al. 2000), or populations selected for improved late reproduction show an increase of lifespan (Rose \& Charlesworth 1980; Luckinbill et al. 1984; Partridge et al. 1999; May 2016). Also direct selection for increased lifespan results in reduced fecundity (Zwaan et al. 1995). Interestingly, the lifespan extension through selection for late reproduction was also associated with an increased resistance to environmental stressors, such as to desiccation, starvation, and ethanol exposure (Service et al. 1985; Rose et al. 1992). This is in line with improved somatic maintenance, at the expense of reproductive output. Furthermore, this relationship between reproduction and lifespan is also observed between individuals in heterogeneous populations of Drosophila melanogaster. There, individual lifespan had a negative correlation with early-life reproduction, but this relationship grows towards a positive relationship between lifespan and late-life reproduction at advanced ages (Curtsinger 2013; Van den Heuvel et al. 2017).

This interrelationship is not only observed in laboratory fly populations, also in human populations similar findings are reported. Women that were able to produce progeny at late age were more likely to become a centenarian (Perls et al. 1997), and in a cohort of British aristocrats that lived before 1700 , the lifespan variations between age 60 and 100 were negatively correlated with the average number of produced progeny. This effect was still present, but reduced for populations after 1700 (Westendorp \& Kirkwood 1998). As these data are in line with the effects observed for the aforementioned laboratory selection experiments and the heterogeneous populations in Drosophila, this suggests a genetic interrelationship between lifespan and reproduction, and points out that lifespan has a strong genetically heritable component that can be exploited by natural selection upon a change of environmental conditions. Thus, ageing evolves as a side-effect of the optimal lifespan-reproduction balance that strongly differs per environment.

\section{GENETIC INTERVENTIONS THAT EXTEND LIFESPAN}

Specific single gene mutations that affect lifespan were first established in the nematode worm Caenorhabditis elegans (Klass 1983), where knockout of the age-1 gene extended lifespan and reduced fertility (Friedman \& Johnson 1988). Since, many more studies have been performed on genetic interventions in the same insulin signalling (IIS) pathway, which showed that other genetic manipulations in IIS extended lifespan in C. elegans as well (Wolkow et al. 2000; Dillin et al. 2002; Libina et al. 2003). Soon genetic research on lifespan in other short-lived model organisms followed, revealing that the same genetic interventions in IIS that extended lifespan in nematodes, also extended lifespan in flies. For instance, similar to its homologue in C. elegans 
(daf-2), partial knockout of the gene encoding insulin receptor (InR) extended lifespan also in flies (Tatar et al. 2001). At the same time, full knockout of chico was investigated, encoding the fly's insulin receptor substrate, and resulted in a lifespan change (Clancy et al. 2001, but see Clancy et al. 2002). Not much later, also rodent lifespan extension was observed upon knockout of insulin receptor (Bluher et al. 2003; Holzenberger et al. 2003) or insulin receptor substrate (Selman et al. 2011). In humans, cohort studies to test the association with longevity have been performed and have also identified significant associations between longevity and IIS signaling, i.e. the FOXO3A locus (Willcox et al. 2008; Flachsbart et al. 2009; Pawlikowska et al. 2009; Soerensen et al. 2010), and the AKT1 locus (Pawlikowska et al. 2009).

The lifespan extension achieved through a lowered IIS activity was however often associated with effects in other life-history traits in the direction of reduced (Darwinian) fitness. For example, reduced IIS activity is associated with delayed development time, a changed body composition, and importantly, reduced reproduction (Friedman \& Johnson 1988; Clancy et al. 2001; Grandison et al. 2009; Gronke et al.2010). This indicates that rather than affecting lifespan only, the IIS pathway has a key modulating function in the organism's whole life-history strategy.

In addition to the IIS pathway, other pathways involved in ageing were also discovered which seem to have similar roles. One example is the Target Of Rapamycin (TOR) pathway in mammals, which interconnects with IIS (Zoncu et al. 2011) and that has genetic orthologues in yeast, C.elegans, and in Drosophila. First, the TOR-pathway was identified for its role in cell growth as TOR inhibition resulted in developmental arrest, reduced reproduction and cell atrophy in C. elegans and Drosophila (Oldham et al. 2000; Jia et al. 2004). But interventions in TOR-signalling also showed an effect on lifespan for different model species, i.e. in baker's yeast (Fabrizio et al. 2001; Kaeberlein et al. 2005), the fungus Podospora anserina (Knupperts et al. 2014), C. elegans (Greer \& Brunet 2009), fruit flies (Bjedov et al. 2010), and mice (Selman et al. 2009). This pathway is of high interest due to the relatively easy manipulation through supplementation of the drug rapamycin that extends lifespan and improves starvation and $\mathrm{H}_{2} \mathrm{O}_{2}$ resistance in flies, but reduces their reproduction and heat resistance (Bjedov et al. 2010; Emran et al. 2014). In line with these effects of IIS/TOR, also reduced juvenile hormone (JH) results in a switch in an insect's life-history and changes the fly's lifespan, reproduction, metabolism, development time, stress response, and behaviour (Flatt et al. 2005; Flatt \& Kawecki 2007; Yamamoto et al. 2013). It is again noteworthy that many interventions that have large effects on lifespan, also result in change of other traits. This is consistent with a key regulatory function for these pathways for multiple processes that are likely genetically connected through evolutionary adaptations, most relevant including the life history configuration. 


\section{DIETARY RESTRICTION}

Lifespan can also be extended by other means than genetic manipulations, for instance through changes in the environment, such as diet and its composition. Maybe one of the most extreme examples of this is illustrated in the honeybee Apis mellifera. The honeybee has two castes, the queen and the worker, but determination of the queen is not genetically determined - workers and queen share the same genotype - but solely by diet. Namely. through feeding a so-called 'royal jelly', larvae develop into queens, which have a larger body size, shorter developmental time, high reproductive ability, and ultimately a lifespan of more than ten times those of worker bees. So by feeding the 'royal jelly' the honey bees' life history dramatically changes (Page \& Peng 2001).

A more intensively studied dietary intervention that also affects an organism's life-history, is dietary restriction (DR), which is generally defined as a reduction of food intake without malnutrition (Weindruch and Walford 1988). DR extends lifespan (and/or increases health) in many organisms from a broad variety of taxa, e.g. in fungi (Jiang et al. 2000; Maas et al. 2004), worms (Klass 1977), spiders (Austad 1989), fruit flies (Chippendale et al. 1993), mice (Weindruch \& Walford 1988), rats (McCay 1935), dogs (Lawler et al. 2008), and even primates (Colman et al. 2009; Mattison et al. 2012). Also in humans, improved health benefits are reported upon DR (Heilbronn et al. 2006; Fontana et al. 2010). However, the magnitude of the lifespan effects of DR range broadly between species, from no effects or a reduced lifespan in some species, for example in the house fly (Cooper et al. 2004) and Ceratitis capitata (Carey et al. 2002), to a lifespan extension of 100-fold, reported for the ageing fungus Podopsora anserina (Maas et al. 2004). Furthermore, lifespan extension by DR often trades-off with an organism's reproduction (Weindruch \& Walford 1988). This combined effect of lifespan and reproduction through DR is considered to be an evolutionary response to periods of food shortage in nature. At the expense of reproduction, it is thought to be more adaptive to temporarily increase investment in somatic maintenance until food becomes more abundant (Harrison \& Archer 1989; Holliday 1989). Consistent with this, DR improves age-associated decline in DNA repair in mice (Weraarchakul et al. 1989), and increased stress resistance and reproductive lifespan in multiple species (Weindruch \& Walford 1988). Typically, DR studies in the laboratory have used a stable food supply throughout life, and may therefore only give limited insight in the actual physiological effects experienced in nature. The food fluctuations studied in the laboratory have showed immediate effects on reproductive performance and mortality in flies (Chippindale $e t$ al. 1993; Good \& Tatar 2001; Mair et al. 2003; Van den Heuvel et al. 2014) and the fungus Podospora anserina (Van Diepeningen et al. 2010a; Van Diepeningen et al. 2014), which is in line with an adaptive response to respond quickly to a changing environment. However, very little research has focussed on unravelling the mechanisms that enable organisms to quickly respond to these environmental fluctuations. Although gene expression studies have been performed on a single diet switch in Drosophila (Gershman et al. 2005; Whitaker et al. 2014), effects of a more natural setting of multiple diet fluctuations throughout life have not been assessed. However, theoretical 
evidence from dynamic state variable models that simulated adaptive strategies in a variable environment is in line with an adaptive response. Namely, in a variable environment it is adaptive to favour investment in maintenance and repair in periods with reduced dietary intake (resulting in a lifespan extension), but when food becomes abundant, investments in reproduction will be favoured (Shanley \& Kirkwood 2000; Mangel 2001; Van den Heuvel 2014).

In addition, other explanations why DR extends lifespan have been proposed. For instance, the sex-specific effects of DR observed in Drosophila (females show a stronger lifespan increase upon DR) (Magwere et al. 2004), raised the idea that a cost of reproduction causes DR to extend lifespan. However, females with a blocked vitellogenesis by the ovo ${ }^{D 1}$ mutation or germline removal by $X$-irradiation did not mitigate the effects of diet on lifespan in Drosophila (Mair et al. 2004). As males have a seemingly smaller nutritional demand to maintain reproduction than females (Wong et al. 2009), the reduced lifespan effects upon diet change in males could alternatively be explained by the differences in nutrient intake, which suggests a detrimental effect associated with food intake. In flies it was shown that mortality trajectories change within $48 \mathrm{hrs}$ upon a switch to DR, which is in line with an immediate removal of an environmental stressor, rather than postponed senescence (Mair et al. 2003). Moreover, by adding the essential amino-acid methionine to a DR-type food resulted in an increase of reproduction without an effect on lifespan (Grandison et al. 2009). This raised the idea that upon DR more amino-acids are consumed than utilized for the fly's reproduction, but these residual amino-acids can be utilized for reproduction when only methionine is add to the diet. Although an increasing food level generally enhances reproduction, upon a severely unbalanced diet this may produce harmful effects on lifespan caused by the large amount of residual amino-acids (Grandison et al. 2009).

Although each of these non-mutually exclusive theories can explain the observed effects of DR on lifespan, more detailed investigation into the exact mechanisms may provide a more profound understanding for how DR extends lifespan. However, despite many developments in genetic research, the exact molecular and genetic mechanisms that mediate the DR response are still poorly understood, as are the relationships between these mechanisms and physiology and ultimately the life history of individuals and species.

\section{GENETIC MECHANISMS MEDIATING THE LIFESPAN RESPONSE TO DR}

Different methods are available to test the genetic mechanisms that mediate the lifespan response to diet, for example, genetic knockouts, RNAi, natural genetic variation, recombinant inbred lines, and gene overexpression are among the tools used. Whether any genetic intervention affects the lifespan response, can assessed by performing a genotype by environment interaction $\left(\mathrm{G}^{\star} \mathrm{E}\right)$ assay. When a $\mathrm{G}^{\star} \mathrm{E}$ interaction is absent, different genotypes will have an identical pattern with a change of diet, in other words the norm of reaction of each genotype run parallel in response to the manipulation of the nutritional environment. For example, when a gene 
knockout extends lifespan, but there is no effect on the diet response, the knockout mutant will show a similar pattern as the wild type strain, with, for instance, a similar lifespan extension at all diet levels (Figure 2a). When the lifespan pattern with diet is shifted, a $G^{\star} E$ interaction is observed. this interaction may enrich functional candidates that are involved in the sensitivity to diet (Figure 2b). In this scenario the lifespan pattern is unchanged; a reduction of food intake still increases lifespan. Therefore, only genotypes that differ in the incline of lifespan, i.e. that cause a flattening of the diet response curve (Figure 2c), are a source for genetic variation in the lifespan response to diet. But to distinguish between these different genotypic effects, dietary responses should be measured on a broad range (Figure 2).

This approach is, however, not always applied in lifespan DR studies, despite its potential to reveal lifespan effects of diet, or to resolve inconsistent outcomes between studies when just a few diets are applied. For example, the effects of chico, a gene coding for the insulin receptor substrate in flies, was first reported to extend lifespan (Clancy et al. 2001), but soon it was discovered that this effect was strongly dependent on the diet range for which lifespan was assessed: lifespan extension changed into a lifespan reduction on the lower nutrition levels (Clancy et al. 2002; i.e. a pattern such as Figure $2 \mathrm{~b}$ was observed). Studies that report an absence of the lifespan response to DR for certain genotypes (or species), typically tested the lifespan effects on a small range of diets (Harper et al. 2006; Sutphin \& Kaeberlein 2008; Liao et al. 2010; Nagakawa et al. 2012; Schleit et al. 2013; Stastna et al. 2015). For example, in mice effects of different recombinant inbred lines (RILs) ranged from no lifespan response, to a reduced or extended lifespan upon DR (Liao et al. 2010), effects that were also observed for natural genetic variation or RILs in C. elegans (Sutphin \& Kaeberlein 2008; Stastna et al. 2015) and RILs in yeast (Schleit et al. 2013) that all were tested on only two diets. In contrast, genotypic effects on $C$. elegans lifespan in response to multiple bacterial concentrations showed clear differences between genotypes, but always a lifespan extension upon DR (Johnson et al. 1990). But also in a more recent study in Drosophila, multiple diets were used and tested the DR response of wildderived populations (Metaxakis \& Partridge 2013). They showed similar effects; natural genetic variation for the lifespan response, but there was always a diet identified on which lifespan was extended upon a reduced diet intake (Metaxakis \& Partridge 2013). These examples illustrate the importance of measuring multiple diets for the right interpretation of whether a DR response is present and of the genetic effects on lifespan. 
a. Parallel response

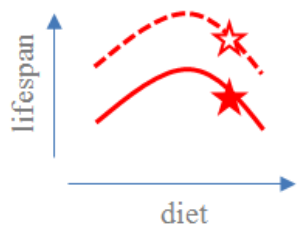

b. Shifted response

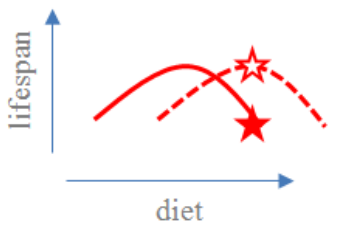

c. Changed response

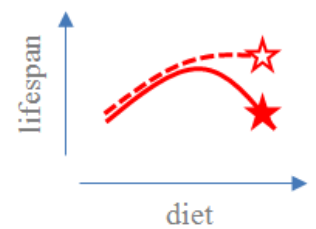

Figure 2: Genotype by diet interactions can vary, but the exact effect can only be observed when a broad range of diets is tested. Namely, when measured on one food type (white versus red star) a similar lifespan extension will be found, despite the fact that the lifespan-diet reaction norm can be very different. These reaction norms can, (a) show parallel patterns with diet change, the genotypic effect causes a lifespan extension without changing the diet response, (b) show a shifted diet response, the genotypic change does not alter the lifespan pattern, but rather the sensitivity to diet change, or (c) show a changed lifespan pattern, where the genotypic change causes a flattening of the lifespan response to diet. Distinguishing between these $\mathrm{G}^{\star} \mathrm{E}$ patterns and the consequences for the mechanisms of a lifespan response to diet requires testing a broader range of diets.

Although applying this approach has revealed a changed diet response by some genetic interventions in the IIS pathway (Figure 2c), studies showed inconsistent outcomes of IIS being involved in the lifespan response to DR. Namely, reduced insulin signalling through partial knockout of genes encoding the insulin receptor $(\operatorname{In} R)$ and/or Drosophila insulin-like peptides (dilps) reduced the effects of lifespan increase through dietary restriction (Grandison et al. 2009; Gronke et al. 2010), whereas manipulations of other IIS components did not result in a changed DR response (Clancy et al. 2001; Min et al. 2008). In C. elegans, genetic effects were shown to largely dependent on the diet regimes used (Greer \& Brunet 2009), and because different laboratories apply different methods to test DR effects (reviewed by: Piper \& Partridge 2007), the reported inconsistencies might be explained by strong interactive effects between dietary methods and IIS manipulation.

A significant aspect to these sometimes contradictory results may be that lifespan extension through diet is not only achieved by reducing the quantity of the diet consumed, but emerging evidence shows that also the combination of different dietary components can affect the lifespan response. For example, in flies it was demonstrated that restricting the calories through reduction of dietary sugar had a much lower lifespan-extending effect than a reduction through dietary yeast (i.e. the source of protein in flies) (Mair et al. 2005).

\section{Geometric framework}

The Geometric Framework (GF) considers nutrition as a multiple-dimension space; different dietary components are represented by different axes and any phenotypic response to these diets is plotted on another separate axis (Simpson and Raubenheimer 1993). Any phenotype can be plotted, such as life span, metabolic parameters, egg laying, or gene expression. This GF approach 
has to date been applied in multiple species, including field crickets (Maklakov et al. 2008), Drosophila (Lee et al. 2008; Skorupa et al. 2009; Bruce et al. 2013), Queensland fruit flies (Fanson et al. 2009), slime molds (Dussutour et al. 2010), and mice (Solon-Biet et al. 2014). The effects of increased diet complexity do not only give a better understanding of how diet affects lifespan, but may also shed light on the inconsistent outcomes between studies, for instance regarding whether IIS is involved in the lifespan extension upon DR.

In Figure 3, GF plots show how nutrient balance may affect lifespan in Drosophila (adapted and changed from: Piper et al. 2011), indicating that lifespan is optimized at low protein/high carbohydrate ratio's (Lee et al. 2008). When a hypothetical genetic intervention shifts lifespan to the right of the plot, i.e. along the protein axis, the interpretation of the effect of the intervention on the diet response will depend on the nutrient-axis under consideration. The example shows that the genetic effect can be perceived to act in a highly diet-independent manner for the one nutrient dimension (Figure 3c), whereas for other nutrient-dimensions it switches the lifespan response (Figure $3 \mathrm{~d}$ ), or even reduces the response (Figure 3e). Although this provides an explanation of the inconsistencies observed between studies, to date $G^{*} E$ effects of this kind have not been tested for a set of natural genotypes or any genetic intervention/mutant.
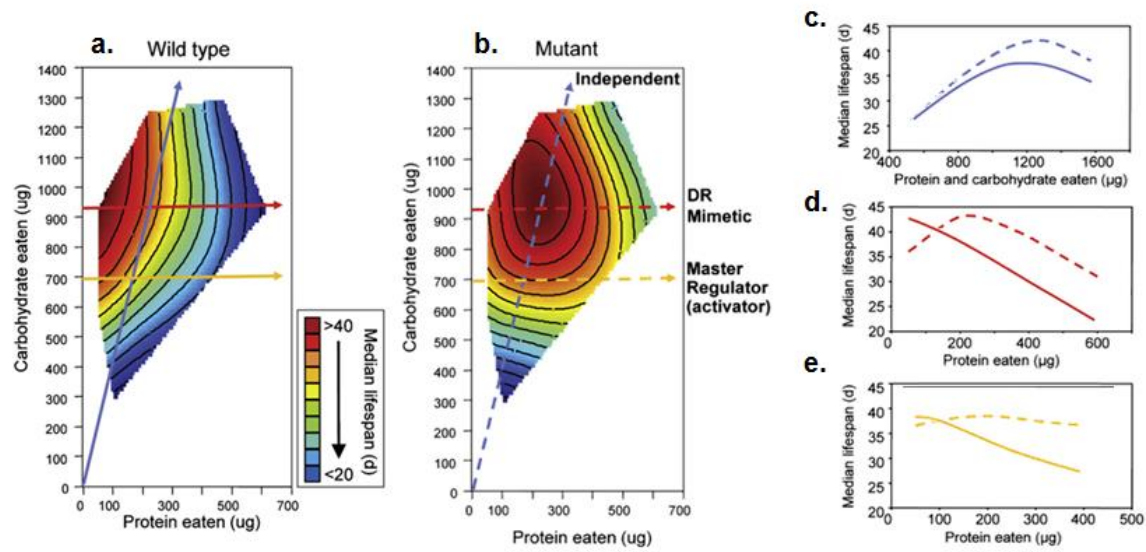

Figure 3: Illustration of how the Geometric Framework (GF) can be used to disentangle the effects and mechanisms of how diet and it components affect phenotypes such as lifespan. Lifespan response surface for wild-type Drosophila (a) and a hypothetical genotype that does not have an altered increase of lifespan but a response shifted to higher protein concentrations (b). For different slices through the response surface, varying interpretations are observed (c-e; indicated by different colours, and genotypes by solid lines for the wild-type and dashed lines for the mutant), similar to the effects demonstrated in Figure 2. Namely, the effects on the mutant range from (c) a nutrient-independent lifespan response for increasing calories (blue colour), to (d) a shifted response with increasing protein on relatively high carbohydrate levels (red colours), or a (e) reduced response with increasing protein on relatively lower carbohydrate levels (yellow colour). Figure is adopted and changed from: Piper et al. (2011). 


\section{Gene expression}

Another method to identify genes involved in the lifespan response to diet is through measuring gene expression of individuals in the different nutritional environments. Measuring wholegenome transcription is an unbiased approach to investigate the association of gene expression with any trait under consideration. By using this technique, an overview of the most important processes up- or downregulated with the intervention can be studied, for the whole organism, specific tissues, and even cells (Berrar et al. 2003). Although the link between expressed genes and protein translation is not always strong (Pradet-Balade et al. 2001), measuring wholegenome expression allows to screen large groups of genes, which makes it suitable for studying complex multigenic processes, such as DR or ageing.

Transcriptional effects of DR have been tested in different species, for example in rhesus monkeys, and genes enriched for processes involved in cytoskeletal organisation and mitochondrial bioenergetics were found (Kayo et al. 2001). In mice, whole-genome expression patterns upon DR were associated with an increased protein turnover and decreased macromolecular damage (Lee et al. 1999). Moreover, the first whole-genome transcription study in flies (Pletcher et al. 2002) identified large and dynamic changing patterns with age and DR, and found genes to be downregulated with DR with a role in cell growth, metabolism, and, interestingly, also reproduction (Pletcher et al. 2002). Although a crude interspecies comparison of transcriptional effects upon DR (i.e. between rats, mice, monkeys, yeast and flies) revealed that no genes and only very few processes (metabolism and cell growth) were shared between different species (Han \& Hickey 2005), however, a systematic analyses of gene expression responses to DR have to date not been performed across species.

Within fruit flies, many whole-genome transcription studies have been performed to investigate the effects of DR, which has uncovered several gene sets that correlated with the flies' lifespan and led subsequently to the identification of causal genes involved in lifespan determination (e.g. Bauer et al. 2010). However, a difficulty with this method is that gene expression is not only sensitive to diet, but also to strain differences (genotypes), gender, type of tissue and age, i.e. factors that often vary between studies investigating the fly's transcription response to DR (e.g. Pletcher et al. 2002; Gershman et al. 2007; Doroszuk et al. 2012; Whitaker et al. 2014; May 2016; Lin et al. 2016; Dobson et al. 2017). These strong methodological and design differences between fly studies provide - without doubt - interesting perspectives on the mechanisms of DR, but it makes it difficult to compare studies, and therefore, confirm the general effects of DR on wholegenome transcription. Additionally, the large number of gene expression changes upon DR makes it difficult to establish which genes have a causal role in the lifespan or in another physiological change that also occurs upon DR, such as locomotion, feeding behaviour, fat storage, and reproduction (Bross et al. 2005; Skorupa et al. 2009; Emran et al. 2014).

The effects of co-varying traits on whole-genome transcription can nevertheless be circumvented by clever study designs, for example by measuring expression upon DR on multiple time points (Pletcher et al. 2002; Doroszuk et al. 2012), or applying more treatments that affect lifespan 
extension through different interventions than only DR (Bauer et al. 2010; Antosh et al. 2011). Also a gene ontology (GO) analysis can be a helpful approach to identify genes or processes involved in the lifespan response. A GO analysis is a method used to reduce the complexity of the information acquired from whole-genome transcription results. Based on database information about gene functions, the large number of significant genes is reduced to a list of enriched processes. Different genes are involved in the same process, which reduces the number of candidate processes through which lifespan is extended upon DR (Huang et al. 2009). Another approach to assign the expression of genes to different co-varying traits is by performing a coexpression analysis. Patterns of co-expression suggest the existence of functional gene networks that are related to the biology of individual traits. Complex traits such as lifespan are polygenic, and it is therefore suggested that a plastic response of such a trait should be achieved through the up- or downregulation of whole gene networks rather than single genes (Mackay 2004). Moreover, a co-expression network assembly can also reveal new transcription factors; namely, each constructed network has highly connected genes that, similar to transcription factors, often have many different target genes (Alic et al. 2011).

So although different challenges arise when measuring whole-genome transcription responses to $\mathrm{DR}$, multiple solutions are available to tackle these problems. Identifying genes involved in lifespan is possible through clever treatment choice that can decouple lifespan and other DRrelated traits, for example through applying a broad range of diets. But clearly, this can also be achieved by performing gene ontology analyses or the construction of co-expression networks, which are examples of analyses that may further improve the functional association of genes with the lifespan response to diet.

\section{THESIS OUTLINE}

The aim of this thesis is to identify genetic mechanisms that are involved in the lifespan response to diet by using two short-lived organisms, the fruit fly Drosophila melanogaster and the fungus Podospora anserina. In the first four experimental chapters I aim to test different genotypic effects on the diet response, through applying the most recent insights of nutritional complexity. In the last two experimental chapters I investigated the fly's whole-genome transcription response to different diet regimes to separately associate gene expression changes with reproduction and lifespan. By using a broad variety of tools and dietary methods, different perspectives on the lifespan response to diet have been acquired.

In Chapter 2 I will test the role of Drosophila insulin like peptides (dilps) in nutrient-dependent ageing by using a Geometric Framework approach. I will assess the effect of a dilp2-3,5 knockout on lifespan and reproduction to verify whether the same knockout has different interpretations for its function in the fly's diet response for different nutrient dimensions. If this is the case, it 

explains the opposing effects observed between studies for the role of reduced insulin signalling in the diet response.

Moreover, in Chapter 3, for the same design, I will measure gene expression responses to nutrients in dilp2-3,5 knockout flies and the control background to test nutrient responsiveness both up- and downstream of the IIS pathway. This may provide more insight in the role of IIS in the response to nutrients, but also in the general dynamics on the pathway level.

Chapter 4 is an exploration of the genetic variation for the response to DR in wild-derived strains of the fungus Podospora anserina. Also here, a broad diet range will be applied to identify different response parameters per strain. Namely, by identifying a differential threshold (e.g. Figure $2 b$ ) or slope (e.g. Figure $2 c$ ) of the diet response between natural strains, I will aim to distinguish between genotypes that change diet sensitivity, or change the lifespan pattern upon diet change. The use of a completely synthetic diet, small nutritional steps along the diet axis between different diets and, importantly, a large array of wild-derived strains, will potentially reveal these and more interesting genotypic effects in the diet response not observed previously in other species and genotype-by-diet investigations.

Through performing experimental evolution (EE) in Drosophila melanogaster I will test in Chapter 5 whether improved reproductive capacity (i.e. local adaptation) to three nutritionally distinct diets affects the flies' lifespan response. This can provide an evolutionary perspective on the connection between reproduction and lifespan, two often interconnected traits in lifespan research (see above). Also in this chapter the $\mathrm{G}^{\star} \mathrm{E}$ interaction will be tested by taking the new insights of the GF into account. By using the same food conditions also applied in Chapters 2 and 3, I will test whether the changes in the genotype composition of the EE populations have affected the (lifespan) responses to DR.

Chapter 6 continues on the genetic effects of lifespan response to diet by exploring the wholegenome transcription responses to a continuously changing environment. This type of fluctuations has been poorly investigated in the laboratory, and may reflect much more the fly's ecological setting than the continuous diet regimes typically applied in DR studies. The reported large plastic response in egg production (Van den Heuvel et al. 2014), suggested a strong adaptive response that quickly altered the life-history as a response to the consumed diet. Thus in this Chapter 6, measuring whole-genome transcription may reveal important mechanistic insights into how these quick alterations will be achieved. The observed variations in reproduction and lifespan between the treatments (Van den Heuvel et al. 2014), may furthermore help to disentangle genetic mechanisms for lifespan from those for reproduction. 
Further investigations into whole-genome transcription will be performed in Chapter 7, where I will test the consistencies between different studies for their whole-genome transcription responses upon DR. Although in flies many studies on whole-genome transcription responses to DR have been performed, a systematic meta-analysis may provide valuable insights into the similarities and differences between studies. In Chapter 7, I aim to investigate what factors may affect whole-genome transcription which should be taken into account for the interpretation of the fly's whole-genome transcription response.

Finally, in Chapter 8, I will synthesize all findings of this thesis and discuss the different insights acquired to provide new views and perspectives useful for future research, but also for the interpretation of past studies on the diet responses of lifespan. I will discuss how genetic change on different levels (gene knockouts, natural genetic variation, and experimental evolution), but also to what extent gene expression can be a useful tool to acquire insights in the mediating mechanisms of the lifespan response to nutrients. 


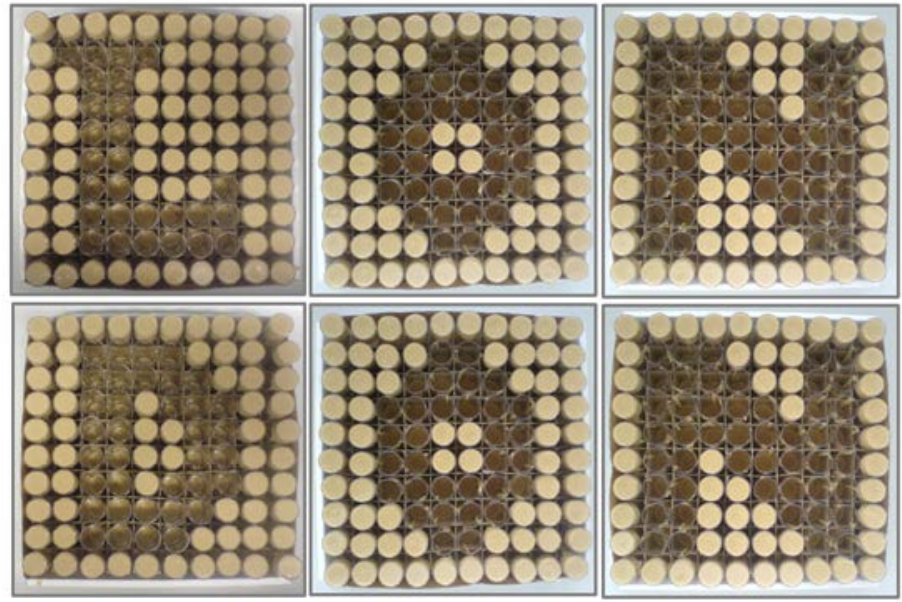

So much food, so many vials... 


\section{CHAPTER II}

\section{BOTH OVERLAPPING AND INDEPENDENT MECHANISMS DETERMINE HOW DIET AND INSULIN-LIGAND KNOCKOUTS EXTEND LIFESPAN OF DROSOPHILA MELANOGASTER}

Jelle Zandveld

Joost van den Heuvel

Bastiaan Johannes Zwaan

Matthew David Woidt Piper

Chapter published in: npj Aging and Mechanisms of Disease 


\section{ABSTRACT}

Lifespan in many organisms, including Drosophila melanogaster, can be increased by reduced insulin-IGF-like signaling (IIS) or by changes in diet. Most studies testing whether IIS is involved in diet-mediated lifespan extension employ only a few diets, but recent data show that a broad range of nutritional environments is required.

Here, we present lifespan data of long-lived Drosophila, lacking three of the eight insulin-like peptides (dilp2-3,5) on nine different diets that surround the optimum for lifespan. Their nutritional content was varied by manipulating sugar and yeast concentrations independently, and thus incorporated changes in both diet restriction and nutrient balance. The mutants were substantially longer-lived than controls on every diet, but the effects on the lifespan response to sugar and yeast differed. Our data illustrate how a greater coverage of diet balance and restriction can unify differing interpretations of how IIS might be involved in the response of lifespan to diet. 


\section{INTRODUCTION}

Lifespan in many organisms, including the fruit fly Drosophila melanogaster, can be increased by genetically reducing activity of the insulin-IGF-like signaling (IIS) pathway and also by a reduction of food intake without malnutrition (dietary restriction, DR) (Fontana et al. 2010; Clancy et al. 2002; Gronke et al. 2010).

Typically, DR involves the use of two or more diets that represent increasing severity of restriction. The lifespan response to these treatments traces an inverted $U$-shape with relatively short life at high food concentrations, ascending to a peak at intermediate food levels, and decreasing again as nutrients become limiting and the organism increasingly suffers from starvation. When analyzing how a long-lived mutant modulates this response, several interacting effects are possible (Piper et al. 2011), but two parameters are thought to be key, (1) does the mutation alter life expectancy attained under each nutritional condition, and, (2) is there a change in the shape in the response of the lifespan to diet? A mutation that attains the first can be argued to extend life, at least in part, through a mechanism independent of DR since it builds on a state in which diet induced longevity is presumed already maximized. However, if a mutation shows the second type of change then it can be interpreted to mediate at least part of the DR response and thus that DR and the mutation are mechanistically linked.

Reports vary on whether or not IIS and DR extend life through an overlapping or independent mechanism (Clancy et al. 2002; Gronke et al. 2010; Min et al. 2008; Grandison et al. 2009; Slack et al. 2011). Reduced IIS by knockout of Drosophila insulin-like peptides (dilps), the fly's homologues for human insulin, has been proposed to mediate the benefits of DR because dietary yeast concentration affects the relative expression of dilp5 (Min et al. 2008). Moreover, knocking out three (dilp2-3,5) of the eight dilps extends lifespan and reduces the magnitude of lifespan change in response to DR (Gronke et al. 2010) - similar to what is observed when overexpressing a dominant negative form of insulin receptor (InR) (Grandison et al. 2009). However, other studies indicate that IIS and DR affect lifespan independently; flies without the IIS transcription factor $d F O X O$ or lacking the insulin receptor substrate (chico) still demonstrate significant lifespan changes in response to DR and do not necessarily extend lifespan beyond that of controls on DR (Clancy et al. 2002; Min et al. 2008; Slack et al. 2011). Thus it is not yet clear to what extent IIS signaling and DR interact and through what mechanism.

Often, the specific set of ingredients and the practices adopted to impose DR are not identical between laboratories ${ }^{8}$ and so DR in one laboratory is likely to be nutritionally different from that in another. Recent studies have revealed that the quantity but also the ratio between dietary protein and carbohydrates can account for the lifespan effects observed under DR (Skorupa et al. 2008; Solon-Biet et al. 2014). Thus, IIS-by-DR interaction studies could yield different outcomes because the mutation may alter the response of lifespan to some nutritional components, but not to others. If true, apparently contradictory outcomes of how a mutant affects the DR response can be resolved when they are viewed as distinct parts of a single lifespan response surface in multidimensional nutrient space (Piper et al. 2011). 
To explore this potential explanation, we measured the lifespan of long-lived dilp2-3,5 mutants and control flies on nine food types, representing all combinations of three yeast and three sugar concentrations (50, 100 and 200 g. $1^{-1}$ each). These diets cover the range of foods used for past studies of IIS-by-DR interactions but extend them by incorporating changes in both diet balance (DB) and diet restriction (DR).

\section{RESULTS \& DISCUSSION}

We found that female dilp2-3,5 mutants were longer lived than controls by at least 21 days on each of our nine food types (Figure 1; Figure 2; Figure S1; Table S1), including the S:Y combination at which lifespan peaks for our control flies ${ }^{9}$. We also found for each diet that reproduction of control flies was higher than that of the mutants (Figure S2, Table S2). Because dilp2-3,5 deletion extended lifespan beyond the value of maximal wild-type longevity attainable through diet manipulation, we conclude this triple knockout extends life, at least in part, by a mechanism independent of that invoked by diet.

The optimal DB was not different for mutants and controls and both genotypes responded similarly to all nutrient manipulations (three-way sugar ${ }^{\star}$ yeast $^{\star}$ genotype interaction, $p>0.1$, cox-proportionalhazard, coxph, Table S2). Genotype did not affect the response of lifespan to yeast ( $p>0.1, c o x p h$, Figure $2 \mathrm{a}, \mathrm{b}, \mathrm{c}$ ), but there was a significant effect of sugar such that high concentrations caused a less severe reduction in lifespan in mutants compared to controls (coxph, $p<0.001$, Table S2, Figure 2 $\mathrm{d}, \mathrm{e}, \mathrm{f})$. Thus, dilps 2,3 and 5 are required for the lifespan reducing effect of increasing sugar levels. Our data thus show that both the modification of diet and deletion of dilps 2,3 and 5 can modify lifespan, and that the mechanisms employed are in some part overlapping, and in some part independent.

This understanding is, however, not clear when we restricted our analysis to important subsets of our diets that represent typical DR experiments. For example, when we compared the effects on lifespan of different subsets of diets the mutants could either reduce the response to DR (Figure 2e, $\mathrm{f}, \mathrm{j}$ ), enhance it (Figure 2d, k) or leave it unchanged (Figure 2a, b , c, l). This same range of interaction responses was found for three sets of isocaloric diets that varied in their S:Y ratios (Figure 2g-i). Importantly, all of these different interventions have previously been grouped under the heading "DR", and yet they are nutritionally different and, yielding non-identical and in some cases, apparently contradictory outcomes.

Both interactive and non-interactive effects of different IIS mutations on DR have been reported (Clancy et al. 2002; Gronke et al. 2010; Min et al. 2008; Grandison et al. 2009; Slack et al. 2011), and we show how these differences can be accounted for by variations in diet regimes. Another likely reason is that each IIS mutation may interact differently with DB and DR. The IIS pathway forms part of a broader nutrient signaling network, which affects lifespan in numerous ways (e.g. TOR suppression) (Hou et al. 2016). Because each component of the canonical IIS pathway is embedded at a different point in this network they may modify the network's overall response to diet in 
different ways. Further work to understand the mechanisms by which diet affects lifespan should incorporate these complex interactions between signaling networks and altered DR and DB.
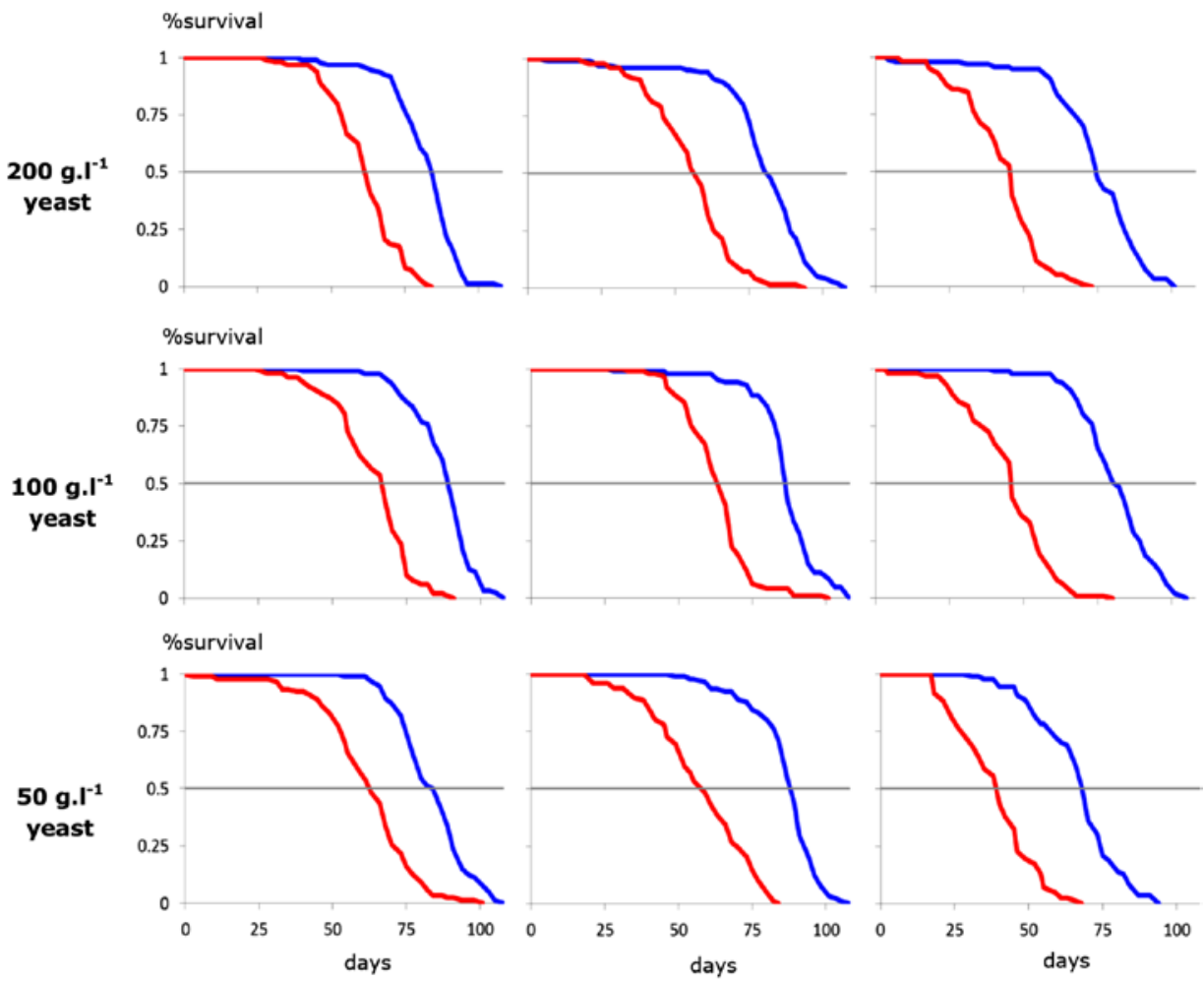

50 g. I $^{-1}$ sugar

$100 \mathrm{~g} . \mathrm{I}^{-1}$ sugar

$200 \mathrm{~g} . \mathrm{l}^{-1}$ sugar

Figure 1: Lifespan curves for dilp2-3,5 mutant (blue) and control flies (red) for all nine food types. On each row the lifespan response to one level of dietary yeast is shown for three different levels of sugar (50, 100 and 200 gr..$^{-1}$ respectively). 

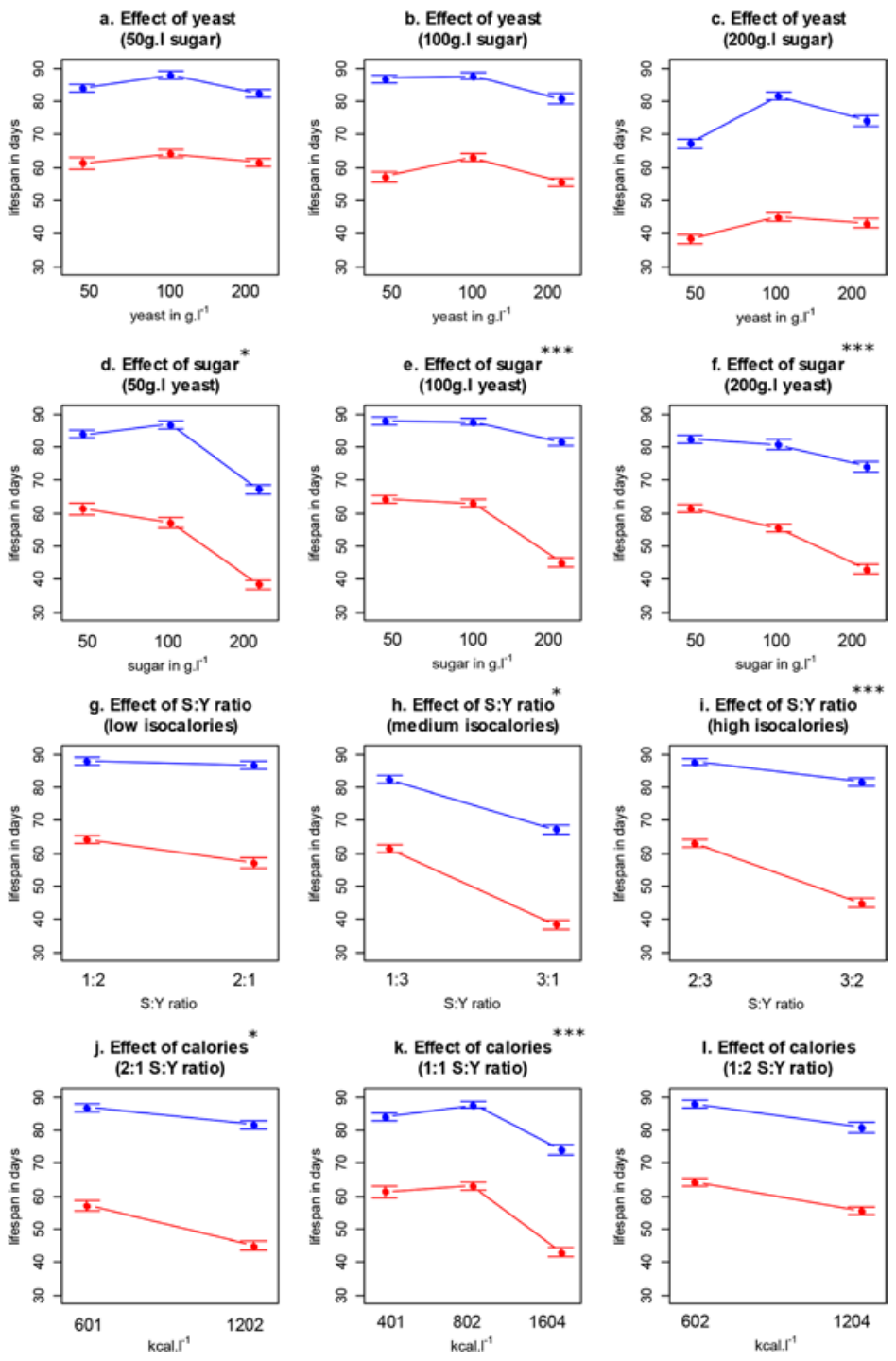

Figure 2: Lifespan interaction plots (mean $+/$ - standard error) display how the lifespan response to nutritionally different DR interventions can be differently affected by the knockout (blue lines represent dilp2-3,5 mutants, red lines control flies); a,b,c, effect of dietary yeast on different sugar levels; d,e,f, effect of dietary sugar on different yeast levels; $\mathrm{g}, \mathrm{h}, \mathrm{i}$, effect of S:Y ratio on different caloric levels; j,k,l, effect of calories on different S:Y ratio's. Asterisks indicate a significant interaction between the dilp2-3,5 knockout and diet regimen under consideration (coxph). " $p<0.05, " * x<0.01, * * * p<0.001$ 


\section{Acknowledgements}

We acknowledge Mingyao Yang, Sahar Emran and Xiaoli He for their help in maintaining the lifespans and fly rearing and we want to thank all members of the Partridge and Gems laboratory and WUR Genetics department for fruitful discussions.

\section{Author contributions}

M.D.W.P and J.Z. designed the experiments. J.Z., J.H., B.J.Z. and M.D.W.P. wrote the manuscript. J.Z. and J.H. performed statistical analysis. J.Z. performed the experiments.

\section{Conflict of interests}

The authors do not have conflicts of interest to declare.

\section{Funding}

Work was funded by the Biotechnology and Biological Sciences Research Council (BB/I011544/1; M.D.W.P), the Royal Society (UF100158 \& RG110303; M.D.W.P), the Australian Research Council (FT150100237; M.D.W.P) by the Network of Excellence LifeSpan (FP6/036894; J.Z., J.H., B.J.Z), and by the European Union's Seventh Framework Program (FP7/2007-2011) under grant agreement number 259679, IDEAL, J.Z., J.H., B.J.Z).

\section{SUPPLEMENTAL MATERIAL}

\section{Figure S1}

Lifespan response surfaces and nutrient orientation of different diet subsets figure 2

\section{Figure S2}

Reproduction patterns of control flies and dilp2-3,5 mutants per food type

\section{Table S1}

Data table phenotypes

\section{Table S2}

Statistical analyses of lifespan and reproduction data

\section{Data S1}

Material and methods 


\section{FIGURE S1}

\section{Lifespan response surfaces and nutrient orientation of different diet subsets figure 2}

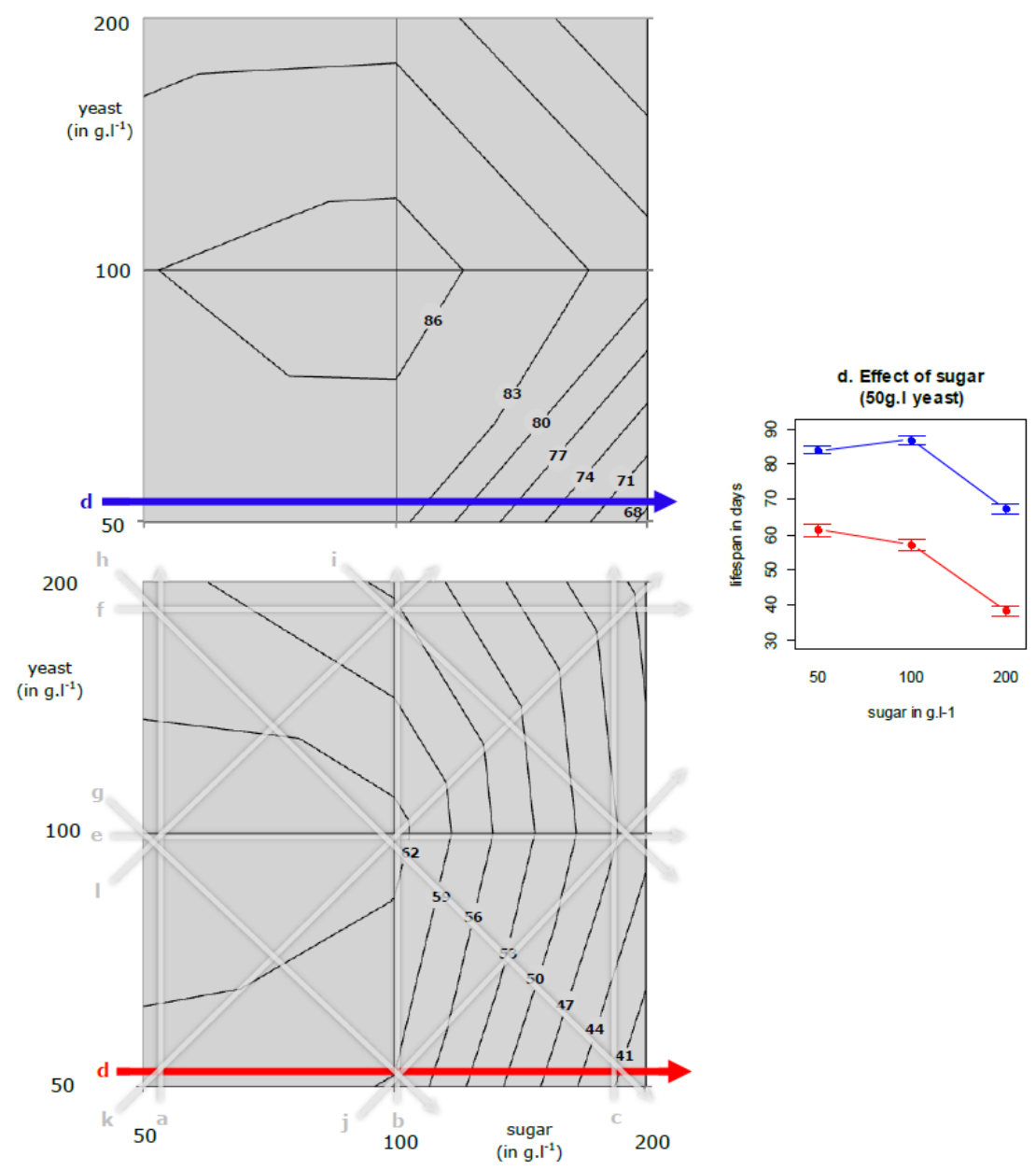

Figure S1: Mean lifespan response surfaces for the nine food types for dilp2-3,5 mutants (up) and control flies (bottom). The arrows display slices through the different nutrient dimensions in figure 2 a-l. For example, a slice through the lowest yeast levels (blue and red arrow for dilp2-3,5 mutants and controls resp.) correspond to the interaction plot of figure $2 \mathrm{~d}$ (right). 


\section{FIGURE S2}

\section{Reproduction patterns of control flies and dilp2-3,5 mutants per food type}

a. $50 S / 200 Y$

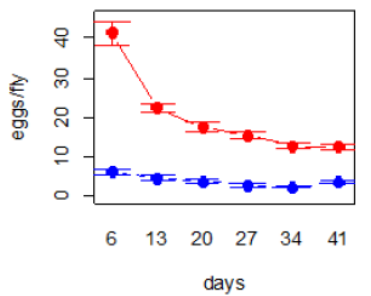

d. $50 \mathrm{~S} / 100 \mathrm{Y}$

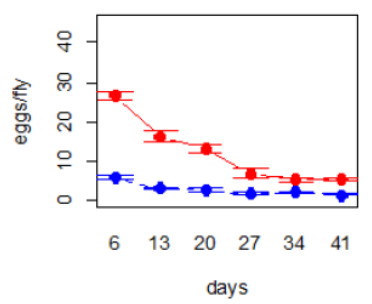

g. $50 \mathrm{~S} / 50 \mathrm{Y}$

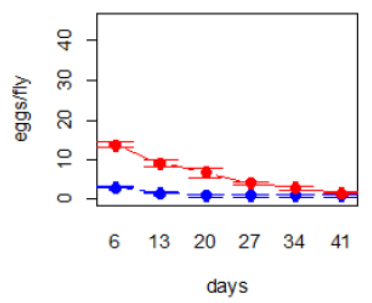

b. $100 \mathrm{~S} / 200 \mathrm{Y}$

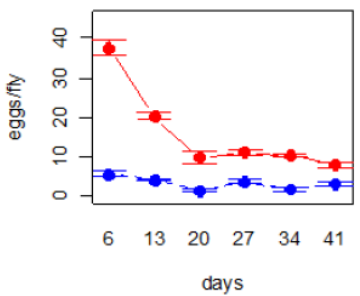

e. $100 \mathrm{~S} / 100 \mathrm{Y}$

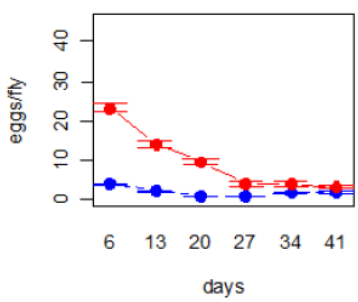

h. $100 \mathrm{~S} / 50 \mathrm{Y}$

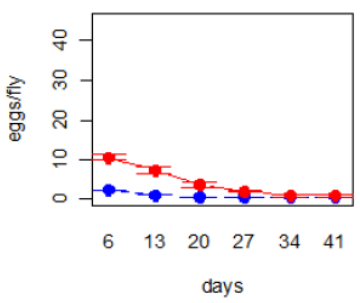

c. $200 \mathrm{~S} / 200 \mathrm{Y}$

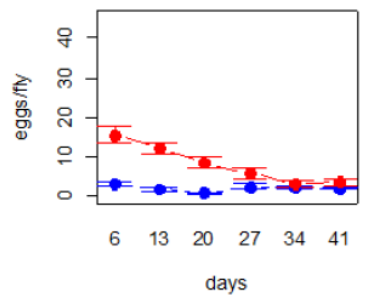

f. $200 \mathrm{~S} / 100 \mathrm{Y}$

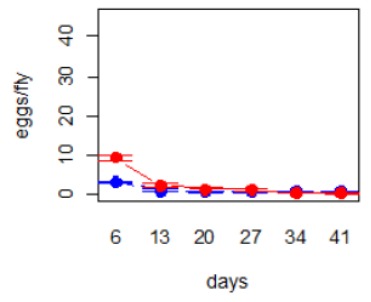

i. $200 \mathrm{~S} / 50 \mathrm{Y}$

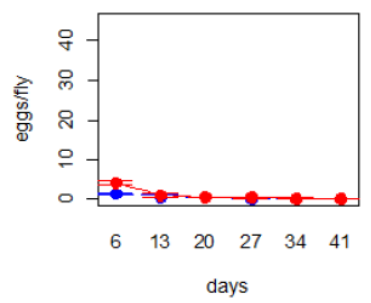

Figure S2.1: Age-dependent reproduction pattern of dilp2-3,5 mutant (blue) and control flies (red) on nine different food type that differ in levels of dietary sugar (S) and yeast (Y) in g.l-1. (a-c) Age-dependent reproduction effects for three different sugar levels and 200g.l-1 yeast, (d-f) 100g.l-1 yeast and (g-i) 50g.l-1 yeast. Points show the mean, error bars represent the standard error. 


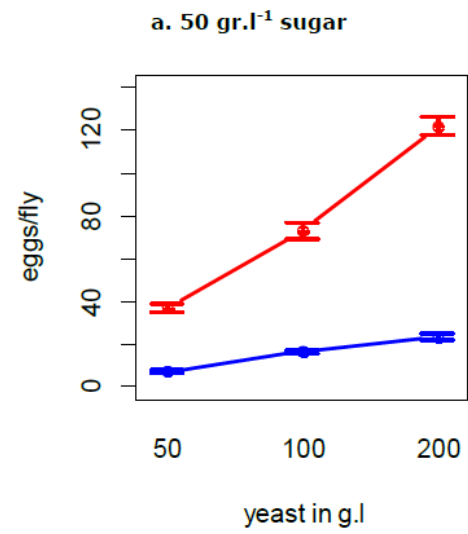

b. $100 \mathrm{gr.I}^{-1}$ sugar

c. 200 gr. $^{-1}$ sugar
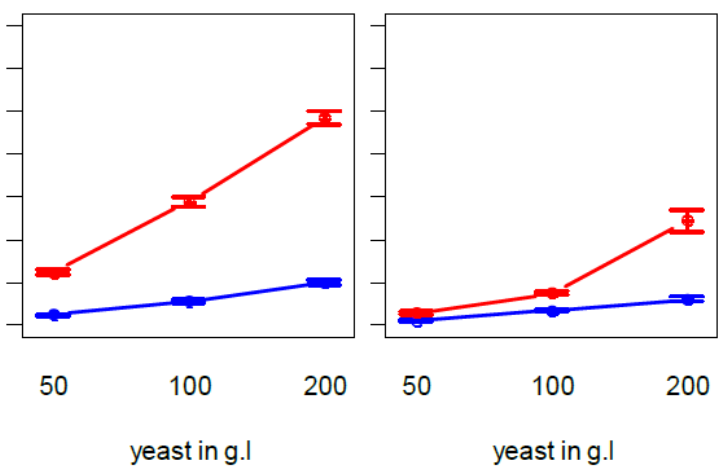

d. 50 gr. $\mathrm{I}^{-1}$ yeast

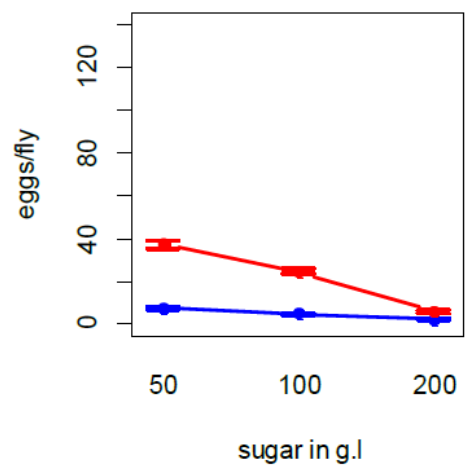

e. 100 gr. $\mathrm{I}^{-1}$ yeast

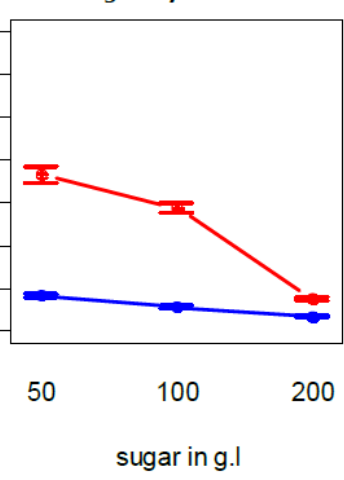

f. 200 gr..$^{-1}$ yeast

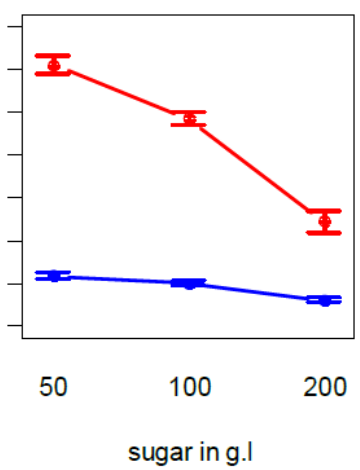

Figure S2.2: Total number of eggs per fly of dilp2-3,5 mutant (blue) and control flies (red) on nine different food type that differ in levels of dietary sugar and yeast. (a-c) Reproduction effects of increasing dietary yeast sorted per level of sugar; (d-f) effects of dietary sugar sorted per level of yeast. Points show the mean, error bars represent the standard error. 


\section{TABLE S1}

\section{Data table phenotypes}

Table S1. Data table phenotypes. Per food type, mean, median, standard error (se) and number of observations (n) for lifespan and reproduction for wild type and dilp2-3,5 mutant flies.

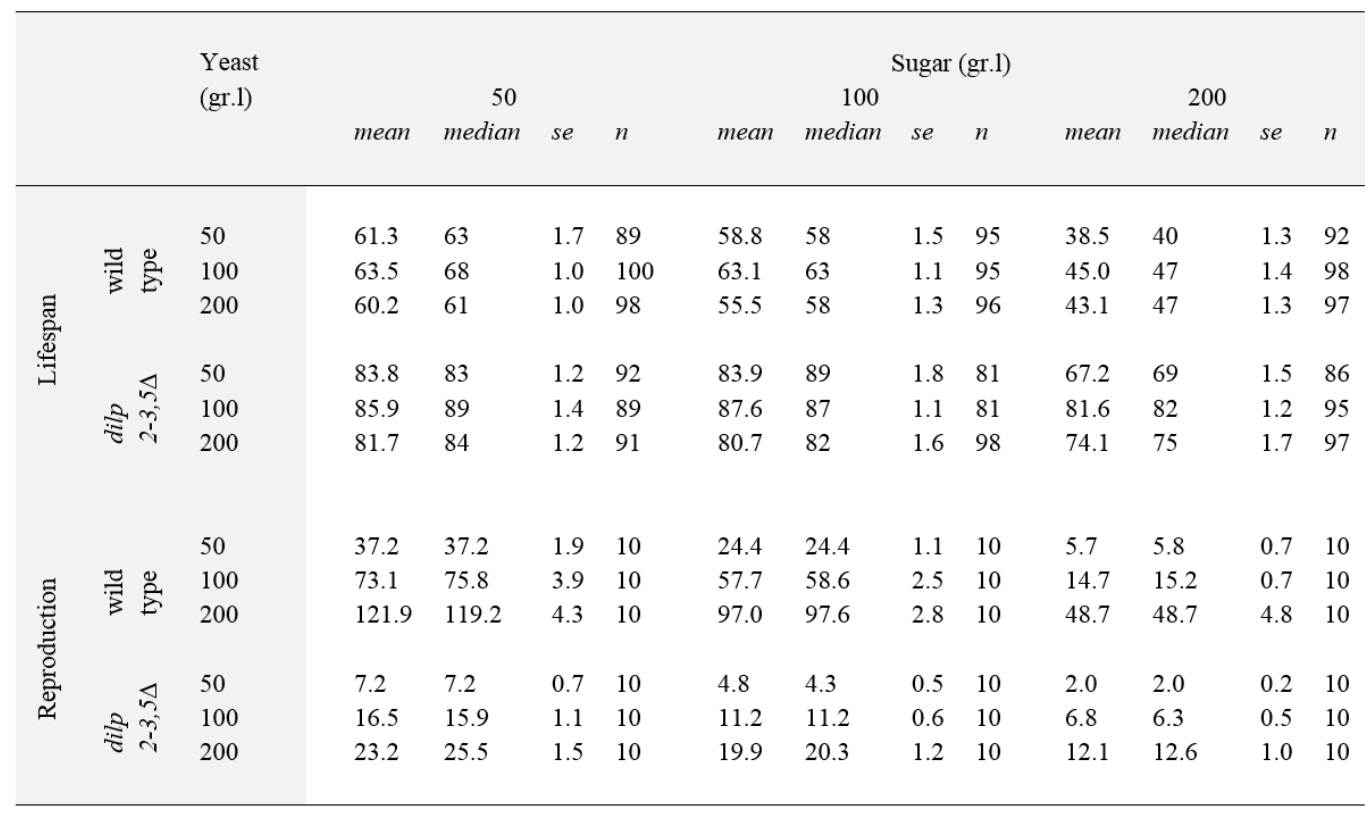




\section{TABLE S2}

\section{Statistical analyses}

Table S2.1: Cox proportional hazard model parameter estimates for effects of the dilp2-3,5 knockout, increasing concentration of dietary sugar (S) and yeast (Y) and their interactions. Per factor the z-value, hazard ratio, 95\% confidence interval and $p$-values is provided. Significance levels: ‘’ $p<0.1 ;{ }^{(*)} p<0.05 ;{ }^{(* *)} p<0.01 ;{ }^{(* * *)} p<0.001$

\begin{tabular}{|c|c|c|c|c|c|c|}
\hline Factor & $z$-value & hazard ratio & Lower .95 & Upper.95 & $\operatorname{Pr}>|z|$ & \\
\hline $\operatorname{dilp} 2-3,5 \Delta$ & -14.561 & 0.188 & 0.150 & 0.235 & $<2 \mathrm{e}-16$ & $* * *$ \\
\hline S100 gr..$^{-1}$ & 2.108 & 1.295 & 1.018 & 1.647 & 0.035 & * \\
\hline S200 gr. $^{-1}$ & 17.459 & 9.446 & 7.341 & 12.154 & $<2 \mathrm{e}-16$ & $* * *$ \\
\hline Y100 gr..$^{-1}$ & 0.244 & 1.030 & 0.814 & 1.303 & 0.808 & \\
\hline Y200 gr..$^{-1}$ & 2.967 & 1.430 & 1.129 & 1.811 & 0.003 & $* *$ \\
\hline S100 gr..$^{-1 \star}$ Y100 gr..$^{-1}$ & -0.507 & 0.927 & 0.692 & 1.243 & 0.612 & \\
\hline S200 gr. $1^{-1 *}$ Y100 gr. $^{-1}{ }^{1}$ & -5.155 & 0.465 & 0.348 & 0.622 & $2.54 \mathrm{e}-07$ & $* * *$ \\
\hline S100 gr. l $^{-1 *}$ Y200 gr. $^{-1}{ }^{-1}$ & -0.300 & 0.957 & 0.717 & 1.277 & 0.765 & \\
\hline S200 gr. $\mathrm{l}^{-1 \star} \mathrm{Y} 200 \mathrm{gr} . \mathrm{l}^{-1}$ & -5.259 & 0.458 & 0.342 & 0.613 & $1.44 \mathrm{e}-07$ & $* * *$ \\
\hline 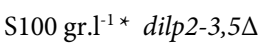 & -2.405 & 0.748 & 0.590 & 0.948 & 0.016 & * \\
\hline S200 gr. $^{-1 *}$ dilp $2-3,5 \Delta^{-1}$ & -7.920 & 0.372 & 0.291 & 0.475 & $2.33 e-15$ & $* * *$ \\
\hline Y100 gr. ${ }^{-1 *} \operatorname{dilp} 2-3,5 \Delta$ & -1.836 & 0.799 & 0.629 & 1.015 & 0.066 & . \\
\hline 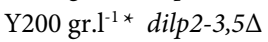 & -1.023 & 0.884 & 0.698 & 1.119 & 0.306 & \\
\hline
\end{tabular}


Table S2.2: Cox proportional hazard p-value estimates between all nine diets within genotype. Diets consisted of all possible concentrations and combinations between 50 gr. $.^{-1}, 100$ gr..$^{-1}$ and 200 gr..$^{-1}$ dietary sucrose (S) or yeast (Y). Significance levels: ‘’ $\mathrm{p}<0.1$; $^{(*)} \mathrm{p}<0.05$; ${ }^{(* *)} \mathrm{p}<0.01$; $^{(* *)} \mathrm{p}<0.001$

\begin{tabular}{|c|c|c|c|c|c|c|c|c|}
\hline & $50 S / 50 Y$ & $50 S / 100 Y$ & $50 S / 200 Y$ & $100 S / 50 Y$ & $100 S / 100 Y$ & $100 S / 200 Y$ & $200 S / 50 Y$ & $200 S / 100 Y$ \\
\hline \multicolumn{9}{|l|}{ wild type } \\
\hline $50 S / 100 Y$ & 0.784 & & & & & & & \\
\hline $50 S / 200 Y$ & 0.141 & $0.035^{*}$ & & & & & & \\
\hline $100 S / 50 Y$ & 0.117 & 0.056 & 0.822 & & & & & \\
\hline $100 S / 100 Y$ & 0.461 & 0.141 & 0.525 & 0.441 & & & & \\
\hline $100 S / 200 Y$ & $0.003^{* *}$ & $0.000 * * *$ & $0.037 *$ & 0.139 & $0.011^{*}$ & & & \\
\hline $200 S / 50 Y$ & $0.000^{\not 6 *}$ & $0.000^{+4 \phi}$ & $0.000^{6.4 \%}$ & $0.000 \$ 6 \%$ & $0.000^{* \phi \phi}$ & $0.000^{*+\phi}$ & & \\
\hline $200 S / 100 Y$ & $0.000 * 4 *$ & $0.000^{*+\%}$ & $0.000^{\circ 4 *}$ & $0.000 \bullet \bullet 4$ & $0.000 * \bullet \bullet$ & $0.000 * * \bullet$ & $0.001 * \psi$ & \\
\hline $200 S / 200 Y$ & $0.000^{* * * *}$ & $0.000 * * *$ & $0.000^{* * * *}$ & $0.000 * * *$ & $0.000^{* * * *}$ & $0.000^{* * *}$ & $0.046 *$ & 0.191 \\
\hline
\end{tabular}

\begin{tabular}{llllllll}
\hline 50S/50Y & $50 S / 100 Y$ & $50 S / 200 Y$ & $100 S / 50 Y$ & $100 S / 100 Y$ & $100 S / 200 Y$ & $200 S / 50 Y$ & $200 S / 100 Y$ \\
\hline
\end{tabular}

dilp $2-3,5 \Delta$

\begin{tabular}{|c|c|c|c|c|c|c|c|}
\hline $50 S / 100 Y$ & 0.092 . & & & & & & \\
\hline $50 S / 200 Y$ & 0.143 & $0.000^{* * *}$ & & & & & \\
\hline $100 S / 50 Y$ & 0.299 & 0.508 & $0.001 \% 4 *$ & & & & \\
\hline $100 \mathrm{~S} / 100 \mathrm{Y}$ & 0.186 & 0.575 & $0.005^{\circ 0}$ & 0.986 & & & \\
\hline $100 S / 200 Y$ & 0.379 & $0.006 * *$ & 0.593 & $0.028 *$ & $0.024 *$ & & \\
\hline $200 S / 50 Y$ & $0.000^{* * * *}$ & $0.000^{*: * *}$ & $0.000 * * *$ & $0.000 * * * *$ & $0.000^{* * *}$ & $0.000 * * *$ & \\
\hline $200 S / 100 Y$ & 0.135 & $0.000 * 0 *$ & 0.779 & $0.000^{* * \psi}$ & $0.006 * \bullet$ & 0.698 & $0.000 * \div \bullet$ \\
\hline $200 S / 200 Y$ & 0.000 *e. & 0.000 to. & 0.000 . & 0.000 *中⿰ & 0.000 * * & 0.000 *0.4 & 0.000 **e \\
\hline
\end{tabular}


Table S2.3 ANOVA model parameter estimates for effects on reproduction of the dilp2-3,5 knockout, increasing concentration of dietary sugar $(\mathrm{S})$ and yeast $(\mathrm{Y})$ and their interactions. F-values, sum of squares, mean square and $p$-values are given. Significance levels: '. $p<0.1$; $^{(*)} p<0.05$; ${ }^{(* *)} p<0.01$; ${ }^{(* * *)} p<0.001$

\begin{tabular}{rcccccc}
\hline Factor & F-value & Sum of sq & Mean sq & $\operatorname{Pr}>|F|$ & \\
\hline Genotype & 1564.014 & 92.88 & 92.88 & $<2 \mathrm{e}-16$ & $* * *$ \\
& & & & & \\
Sugar & 406.860 & 48.32 & 24.16 & $<2 \mathrm{e}-16$ & $* * *$ \\
Yeast & 599.170 & 71.16 & 35.58 & $<2 \mathrm{e}-16$ & $* * *$ \\
& & & & & \\
Sugar $^{*}$ Yeast & 13.935 & 3.31 & 0.83 & $8.60 \mathrm{e}-10$ & $* * *$ \\
Sugar $^{*}$ Genotype & 26.587 & 3.16 & 1.58 & $1.03 \mathrm{e}-10$ & $* *$ \\
Yeast $^{*}$ Genotype & 3.644 & 0.43 & 0.22 & 0.028 & $*$ \\
& & & & & \\
Sugar $^{\star}$ Yeast $^{\star}$ Genotype & 2.440 & 0.58 & 0.14 & 0.049 & $*$ \\
\hline
\end{tabular}




\section{DATA $S 1$}

\section{Materials and Methods}

\section{Fly stocks and husbandry}

Dilp2-3,5 mutants were backcrossed for at least 6 generations into the control fly background, outbred Dahomey flies carrying the $w 1118$ mutation (Gronke et al. 2010) and kept in population bottles, being refreshed with an additional 2 generations of backcrossing every 6 months. This experiment was performed in 2012. The control flies were housed in population cages with overlapping generations and maintained at standard density in bottles for two generations before the start of the experiment. The experiment was performed at $25^{\circ} \mathrm{C}, 12$-hour light/dark cycle at $65 \%$ humidity on once-mated female flies. Flies were reared at standard density according to the method of Clancy et al. (2001), and developed for 10 days on standard food (1SY-A). After emergence, adult flies were transferred to fresh 1SY-A bottles to mate for 48 hours. Under $\mathrm{CO}_{2}$ anesthesia female flies were collected and allocated at a density of 10 flies into vials containing one of the nine food treatments.

\section{Food treatments}

For all food types per liter the following ingredients were added: $15 \mathrm{~g}$ agar (Sigma, Dorset, UK), $3 \mathrm{ml}$ propionic acid (Sigma, Dorset, UK), $30 \mathrm{ml}$ Nipagin (Clariant UK Ltd, Pontypridd, UK) in 95\% ethanol. The quantities of autolysed Brewer's Yeast (MP Biomedicals, Solon, OH) and sucrose (Tate \& Lyle sugars, London, UK) varied between the food types. Standard food (1SY-A) consisted of 50 gr..$^{-1}$ sucrose and 100 gr..$^{-1}$ autolysed yeast. The nine food varied in the combination of sucrose and yeast concentrations as in Skorupa et al. (2008), but at concentrations of 50 gr. $\mathrm{l}^{-1}, 100 \mathrm{gr} . \mathrm{l}^{-1}$, and 200 gr..$^{-1}$; these diets were chosen because they encompass the S/Y concentrations that maximized lifespan in Bass et al (2007) and Skorupa et al. (2008), studies which we aimed to use as benchmarks.

\section{Reproduction assay}

Reproduction was determined by counting the number of eggs laid in 24 hrs per vial. Eggs were counted every week for the first six weeks of adult life. Per week the average egg count per female per vial was calculated and used in the analyses

\section{Lifespan assay}

For lifespan analysis three times per week flies were placed onto fresh media and for each transfer deaths were recorded.

\section{Statistical analyses}

To test lifespan effects of, and interactions, between genotype, sucrose and yeast concentration, a cox proportional hazard test was performed. For reproduction, the total egg count data for each vial was summed and log transformed. An analysis of variance (Anova) was performed to test for effects 
of and interactions between genotype, sucrose and yeast concentration, a t-test was performed to test for differences between single diet treatments within genotype.

\section{References Materials and Methods}

Bass TM, Grandison RC, Wong R, Martinez P, Partridge L, Piper MDW. Optimization of Dietary Restriction Protocols in Drosophila. J Geront. 2007; 10; 1071-1081

Clancy DJ, Kennington WJ. A simple method to achieve consistent larval density in bottle cultures. Dros. Inf. Serv. 2001; 84: 168-169.

Gronke S, Clarke DF, Broughton S, Andrews TD, Partridge L Molecular evolution and functional characterisation of Drosophila insulin-like peptides. PLoS Genet. 2010; 6: e1000857

Skorupa DA, Dervisefendic A, Zwiener J, Pletcher SD. Dietary composition specifies consumption, obesity, and lifespan in Drosophila melanogaster. Aging Cell 2008; 7: 478-490. 


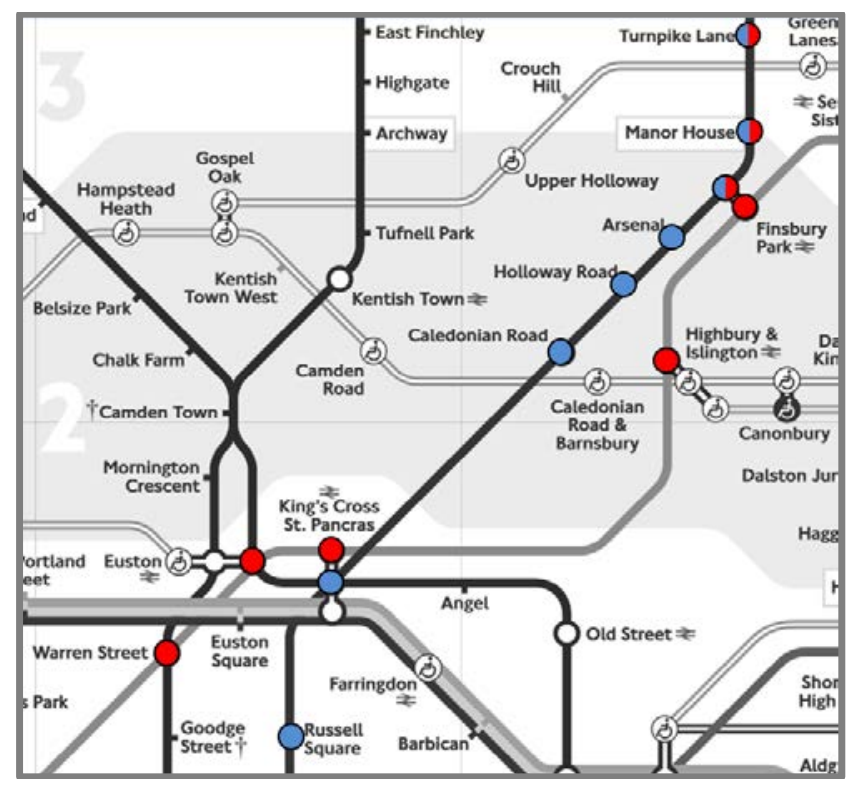

Tube connections to travel between 78 a Turnpike lane and the Darwin building, Gower street, London 


\section{CHAPTER III}

GENE EXPRESSION RESPONSES UPON KNOCKOUT OF INSULINLIKE PEPTIDES IN DROSOPHILA MELANOGASTER

Jelle Zandveld Joost van den Heuvel Matthew David Woidt Piper

Bastiaan Johannes Zwaan 


\section{ABSTRACT}

Lifespan in many organisms, including the vinegar fly Drosophila melanogaster, is increased by both lowering insulin-IGF signaling (IIS) activity, and also by changes in the dietary balance (often referred to as dietary restriction (DR). Previous work showed that dilp2-3,5 mutants were long-lived under a broad range of dietary conditions, but whether a typical DR response was observed was depending on the yeast or sugar dimension of the diet. Moreover, it is unclear whether the gene knockout and DR effects are mediated via the same gene expression differences. Therefore, in this study we assessed the effects of this life-extending manipulation on gene expression responses to diet for up- and downstream components of the insulin-IGF signaling (IIS) pathway. We showed that expression of dilps in control flies responded to both dietary sugar and/or yeast changes and that these patterns correlated to diet effects of lifespan or reproduction. Interestingly, dilp6 expression pattern changed upon dilp2-3,5 deletion and became responsive to high yeast levels in the mutants. As the downstream gene $4 e-b p$ maintained its nutrient responsiveness in the mutants, we discuss that this data is consistent with a nutrientdependent compensation of dilp6 for the absence of dilps that together with interconnected pathways may not avoid lifespan to increase, but maintain responsiveness to particular nutrient dimensions in dilp2-3,5 mutants 


\section{INTRODUCTION}

Lifespan in many organisms, including fungi, worms, insects, rodents, and primates can be increased by dietary restriction, a reduced food intake without malnutrition (Fontana et al. 2010). Similarly, the insulin-IGF signaling (IIS) pathway has a role in health and longevity in many organisms, perhaps including humans (Fontana et al. 2010). In the fruit fly Drosophila melanogaster, both dietary restriction and genetic modifications of IIS increases lifespan (Clancy et al. 2002; Tatar et al. 2001; Grandison et al. 2009; Gronke et al. 2010), but addressing the question whether interventions in IIS and diet extend lifespan through dependent or independent mechanisms gives contrasting results (Clancy et al. 2002; Tatar et al. 2001; Grandison et al. 2009; Gronke et al. 2010).

Recently, in more detailed studies, it has been shown that a lifespan extension by diet is rather an effect of changing the balance of (macro)nutrients in the food, than only nutrient concentration (Mair et al. 2005; Lee et al. 2008; Skorupa et al. 2008; Solon-Biet et al. 2014, Piper et al. 2017). These new insights have largely been derived from work using the 'Geometric Framework' (GF) in which diet change is regarded as a multidimensional nutritional environment (Simpson \& Rauenheimer, 1993). The implication is that when testing effects of diet, a broad range of food types should be applied that differs in both concentration and composition of macronutrients (Simpson \& Rauenheimer, 1993).

Potential mediators of how diet affects lifespan are the eight Drosophila insulin-like peptides (DILPs), circulating ligands homologous to human insulin (Slaidina et al. 2009; Okamoto et al. 2009; Colombani et al. 2012). Expression of specific dilps is tissue and stage-dependent (Slaidina et al. 2009; Okamoto et al. 2009; Colombani et al. 2012) and in adults it has been shown that the expression of dilps is affected by the manipulation of the diet, be it only for yeast or for dietary all dietary components (Broughton et al. 2005; Min et al. 2008). Recently it was shown that macronutrient combinations known to alter lifespan or reproduction also changes relative expression of different dilps, and also that the expression patterns of dilp2,-3,-4 and -5 correlated with the flies' lifespan and/or reproduction response to nutrients (Post \& Tatar, 2016). Furthermore, lifespan was extended in flies overexpressing dilp6 in fat body (Bai et al. 2012) and also in mutants with a knockout of dilp2 singly, dilp 2 \& 3 together, or triple knockout of dilps 2, 3 and 5 (not including dilp6; Gronke et al. 2010). Altogether, these data point towards for dilps mediating the effects of diet balance on longevity.

In our previous work we showed large effects of dilp2-3,5 deletion to extend lifespan and reduce reproduction, confirming previous reports, but whether these insulins mediated the lifespan response to diet, depended on the specific macronutrient changes under consideration. For example, dilp2-3,5 mutants had a changed lifespan response to high sugar levels, but did not change their response to yeast (Zandveld et al. 2017). Interestingly, the expression patterns of dilps involves compensatory regulation (Gronke et al. 2010), and, conversely, a downregulation of the other dilps is observed when in adult fat bodies dilp6 is overexpressed (Bai et al. 2012). Although 
other interweaved pathways should also be taken into consideration (Kafri et al. 2009; Emran et al. 2014), these data suggests that by upregulating other dilps compensation for the loss of specific dilp expression is realized, possibly aimed at maintaining nutrient homeostasis across diet dimensions. This appears to be realistic scenario since dilp2-3,5 knockout flies maintained the lifespan response for certain diet dimensions (Zandveld et al. 2017).

To explore this further, we focused in this study on the effects of dilp gene expression of the longlived dilp2-3,5 knockout flies and their controls across nine food types that include a broad range of lifespan responses. We aimed to measure gene expression changes upon dilp2-3,5 knockout, and thereby contributing to the understanding of the role of dilps in mediating the fly's diet responses.

\section{MATERIALS AND METHODS}

\section{Fly strains and husbandry}

In 2011, dilp2-3,5 mutants were backcrossed into the control fly background, outbred Dahomey flies carrying the $w 1118$ mutation (Gronke et al. 2010) and kept in population bottles. The control flies were maintained in population cages with overlapping generations and kept in bottles for two generations before the setup of our experiments. The experiment was performed at $25^{\circ} \mathrm{C}, 12$-hour light/ dark cycle at $65 \%$ humidity on once-mated female flies. Flies were reared on standard food (1SY-A)at standard densities according to the method of Clancy et al. (2001). After emergence, adult flies were transferred to fresh 1SY-A bottles to mate for 48 hours. Under $\mathrm{CO}_{2}$ anesthesia female flies distributed over vials at a density of 10 flies containing one of the nine food treatments for a total of 12 vials per food treatment (thus 120 once-mated female flies for each treatment group).

\section{Lifespan and reproduction}

The lifespan data in this study has been published previously (Zandveld et al. 2017, Chapter 2 this thesis) and is solely used in this study for correlating the gene expression (RT-qPCR) data with the life history phenotypes of lifespan and reproduction. Female reproduction was determined by counting the total number of eggs laid per vial in the 24 hours prior to sacrifice on day 7 .

\section{Fly media preparation}

For all food types standard ingredients were added per litre, $15 \mathrm{~g}$ agar (Sigma, Dorset, UK), $3 \mathrm{ml}$ propionic acid (Sigma, Dorset, UK), $30 \mathrm{ml}$ Nipagin (Clariant UK Ltd, Pontypridd, UK) in 95\% ethanol, but the quantities of autolysed Brewer's Yeast (MP Biomedicals, Solon, OH) and sucrose (Tate \& Lyle sugars, London, UK) differed between diet treatments. Standard food (1SY-A) consisted of 50 gr..$^{-1}$ sucrose and 100 gr..$^{-1}$ autolysed yeast. The nine food varied in the combination of sucrose and yeast concentrations following Skorupa et al. (2008), and varied between 50 gr. $\mathrm{l}^{-1}$, 100 gr. $^{-1}$ and 200 gr..$^{-1}$. 


\section{RT-qPCR}

After 7 days on their food treatment, flies were snap frozen in liquid nitrogen. Flies of two vials were combined and RNA of 20 heads and bodies was extracted for a total of six replicates per treatment per genotype. For heads, RNA extraction, cDNA synthesis, and qPCR was performed as in Broughton et al. (2005). For bodies, RNA was extracted using a RNAeasy kit (Qiagen), RNA was reverse transcribed using the iScript cDNA synthesis kit (Bio-Rad) and qPCR's were performed with iQSYBR Green Supermix (Bio-Rad) on a CFX96 Touch $^{\text {mi }}$ Real-Time PCR Detection System (Bio-Rad). Relative transcript levels were determined (standard curve method) and normalized to act- $5 c$ in heads. In bodies, act- $5 c$ was affected by food treatment in bodies (data not shown), therefore, in bodies expression was measured relative to $\delta$-tubulin. All expression data was normalized according to the method of Pfaffl (2001). Primers sequences are provided in Table S1.

\section{Analyses and Statistics}

To create response surface figures per phenotype the mean of each of the nine diets was entered in a spreadsheet programme (MS Excel ${ }^{(\mathrm{R})}$ ) and plotted using the surface plotting option.

Expression values were log transformed and a 'linear mixed effects model' (lmerTest package in $R$ ) was used, with 'RNA extraction set' as a random factor. For post-hoc tests, we used the function 'General Linear Hypotheses' (multcomp package in R). 


\section{RESULTS}

Previously we reported that lifespan of dilp2-3,5 mutants was extended irrespective of dietary nutrient manipulations, showing a role of dilps in ageing. Whether we observed that the mutations altered the lifespan response to diet depended on the diet orientation considered; for example, mutant flies showed no difference in their response to dietary yeast (Zandveld et al. 2017, Figure S1). To assess the potential involvement of dilps in mediating these lifespan and reproduction response to altered nutrition, we first measured the transcript levels for the dilp2-3,5 genes on each of the nine diets in heads of control flies (Figure 1) and correlated it to their lifespan phenotype (lifespan data from Zandveld et al. 2017) and to the reproduction pattern at the moment of sampling for each of the diets (Figure S1).
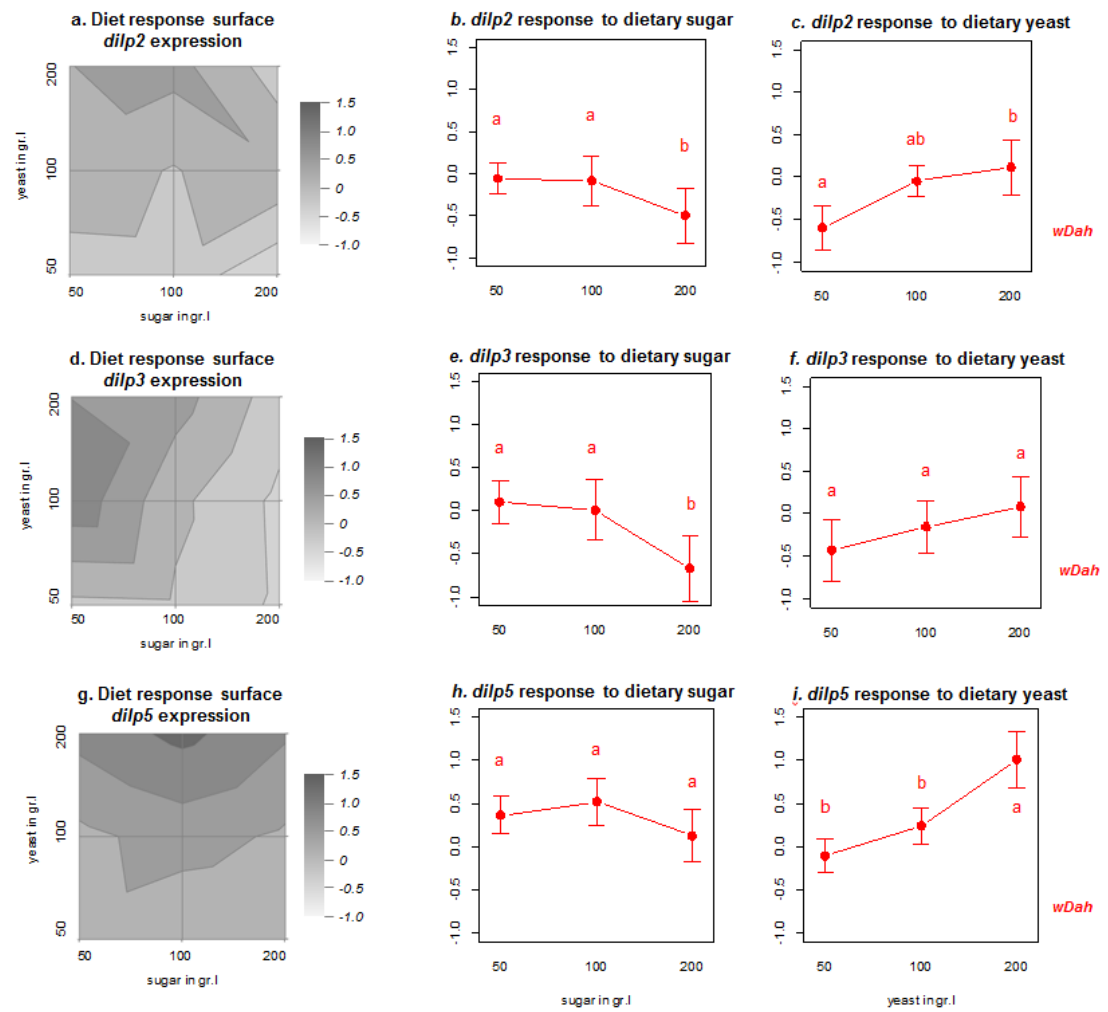

Figure 1: Diet response surfaces (left) and corresponding line plots (right) for effects of dietary sugar and yeast in the relative expression of $(\mathrm{a}-\mathrm{c}) \operatorname{dilp} 2,(\mathrm{~d}-\mathrm{f}) \operatorname{dilp} 3$ and $(\mathrm{g}-\mathrm{i})$ dilp 5 versus Actin $-5 \mathrm{c}$ in heads of $w D a h$ flies. In the line plots letters indicate a significant difference between the diet levels $(l m e r, p<0.05)$

In Figure 1 we show the relative expression of $d i l p 2,-3,-5$ in heads of control flies. Expression of all three dilps was significantly elevated with increasing yeast levels (lmer, $p<0.01$ ), and dilp2 and - 
3 also showed a significant elevated expression on low sugar (lmer, $p<0.01$, Figure 1$)$. When we correlated the transcript levels to the flies' lifespan and reproduction pattern, we observed that the expression patterns of $d i l p 2$ and dilp 3 showed correlations with reproduction or lifespan (Figure S2). Although rough associations, both relative expression of dilp2 showed a positive relationship with the reproduction response (dilp2: $R^{2}=0.08, p<0.05 ;$ dilp3: $R^{2}=0.06, p<0.1$ ). Furthermore, dilp3 had a positive, and nearly significant correlation with lifespan $\left(R^{2}=0.06, p<0.1\right)$. Expression of dilp5 was significantly affected by increasing dietary yeast (lmer, $p<0.01)$ and showed a significant correlation with the reproduction response to diet $\left(R^{2}=0.12, p<0.05\right.$; figure $\left.\mathrm{S} 2\right)$. In the control flies, dilp6 expression was not affected by changes in dietary sugar (lmer, $p>0.1$ ), and showed an increased expression on $100 \mathrm{gr} . \mathrm{l}^{-1}$ yeast (lmer, $\left.p<0.05\right)$. No correlations between relative expression of dilp6 and lifespan nor reproduction were found (Figure S2).

In summary, we showed that relative expression of head-expressed dilps responded to diet variations, and has associations with the diet response of lifespan and/or reproduction.

\section{Effects of dilp2-3,5 knockout on gene expression patterns with diet}

Genetic interventions to modify expression of selected dilps have shown compensatory expression profiles of the remaining dilps (Gronke et al. 2010; Bai et al. 2012), but the interactions of these changes with nutritional variations are not known. By measuring expression of dilp6 in heads of flies of dilp2-3,5 mutants on a broad range of food types, we observed that dilp6 expression significantly changed its response to diet changes upon the triple knockout (lmer, $p=0.01$ ). Namely, where in control flies relative expression of dilp6 was highest on intermediate yeast concentrations, in the mutants, dilp6 expression peaked on the highest yeast levels. On intermediate dietary yeast dilp6 expression was not changed upon knockout (lmer, $p=0.1$ ). Therefore, the gene expression effect of dilp6 upon knocking out dilp2-3,5 depends on diet type under consideration (Figure 2). If this new dilp6 responsiveness can be seen as a mechanism to maintain systemic IIS in the absence of dilp2, -3 and -5, the cell non-autonomous effects of IIS in response to diet should be unchanged in control and dilp2-3,5 knockouts. We therefore measured expression of $4 e-b p$, a downstream IIS target gene, across all food types in bodies of both genotypes. Consistent with a diet-independent effect of the triple knockout, expression of $4 e-b p$ was lower on all diets in dilp2-3,5 knockout flies compared to controls (lmer, $p<0.001$ ). The responsiveness of $4 e-b p$ to nutrients was, however, not different for the two genotypes (lmer, $p>0.1$ ). Both controls and dilp2-3,5 mutants showed no significant response of $4 e-b p$ to altered sugar, and an increased expression with reduced levels of dietary yeast (lmer, $p<0.05$ ). If IIS is responsible for modifying $4 e$ - $b p$ expression in response to diet, these data suggest that other components of IIS could compensate to maintain the downstream nutrient homeostasis for the nutritional changes we tested. Because the lifespan and reproduction responses of dilp2-3,5 mutants were modified in response to some nutrient dimensions compared to control flies (Zandveld et al. 2017), full compensation for the loss of the three dilps could apparently not be achieved. 


\section{DISCUSSION}

In this study we explored the effects of dilp gene expression in heads and bodies of the long-lived dilp2-3,5 knockout mutants and control flies. We showed that upon knockout of the three nutrient-responsive dilps, the next highest expressed dilp in brains of fruit flies, dilp6, became responsive to higher yeast levels (Figure 2). As dilp2-3,5 mutants in our previous study showed not changed lifespan response to differential yeast levels (Zandveld et al. 2017), these findings are in line with a compensatory response for the loss of the three dilps.

Expression of dilps responds to diet and correlates to the fly's lifespan and reproduction response We observed in control flies that expression of $\operatorname{dilp} 2,3$, and 5 responded to both yeast and sugar (Figure 1), showing patterns that correlate to the lifespan and/or reproduction response of the flies (Figure S2). Previous studies that focused on the expression of dilps in heads using fewer dietary combinations (changing only the yeast component of the diet) showed that increasing dietary yeast changed expression of dilp5, but not of dilp2 and dilp3 (Min et al. 2008; Broughton et al. 2010). In a more comparable dietary set up, also using a broad range of diets, many similarities in the expression change of dilps with diet were observed (Post \& Tatar, 2016). Although Post \& Tatar measured expression of dilps in whole flies, their results showed clear similarities to our results in fly heads, most likely because apart from dilp6, most other dilps are predominantly expressed in fly heads (FlyAtlas). We confirmed the previous observed upregulation of dilp2 with high yeast levels (figure 1), but did not replicate the reported increased expression of dilp2 also with low yeast levels (Post \& Tatar, 2016). Expression of dilp6 in bodies was consistent with previous results, showing in bodies an increase with lower yeast levels (Figure S3). For expression of dilp6 in heads, to our knowledge, no other studies were performed. Most notably, we confirmed the previous expression responses of $d i l p 3$ and dilp 5 to changes in dietary yeast and sugar (Figure 1), as well as the correlations with the lifespan and reproduction phenotypes (Figure S2).

Dilp expression in mutants: a compensatory role for dilp6?

As expected, we were unable to measure effects of dilp2, -3 or -5 expression in the mutants (data not shown), but measured expression of dilp6 in heads of both mutants and control flies. And we observed dilp6 had a higher expression on intermediate yeast levels in heads of control flies, which disappeared in dilp2-3,5 mutants, were dilp6 became responsive to higher yeast (Figure 2). Upregulation of other dilps in response to loss of the three insulin-like ligands have been reported previously. Namely, a downregulation of dilp2 and -5 was observed upon overexpression of dilp6 in adult fat bodies (Bai et al,. 2012). And, expression of other dilps was upregulated upon ablation of dilp producing cells and genetic knockouts for single, double, or triple dilps (Broughton et al. 2008; Gronke et al. 2010). We showed that investigating expression changes on a limited range of diets is likely not capturing the full extent of this seemingly compensatory response. Our observed upregulation of dilp6 in the mutants is consistent with these previous results, possibly compensating for the loss of dilps in the central regulation. But importantly, because we applied a 
broad range of diets, we showed these changes have a diet-dependent character (Figure 2), maintaining an upstream nutrient response within the IIS pathway.
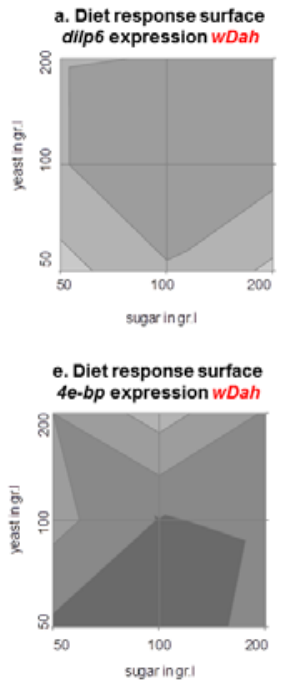
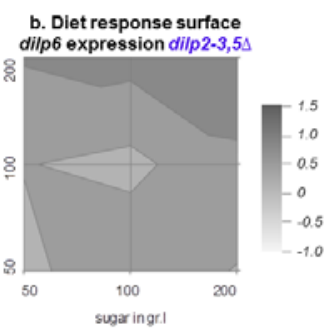

f. Diet response surface
4e-bp expression dilp $2-3,5$

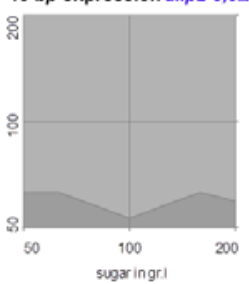

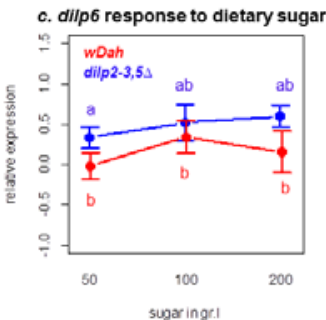
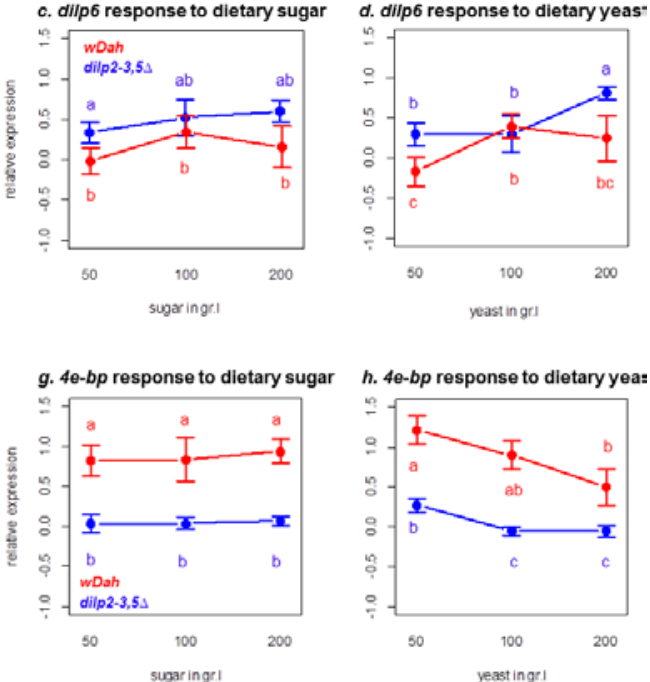

Figure 2: Diet response surfaces (left) and corresponding interaction plots (right) for effects of dietary sugar and yeast in the relative expression of (a-d) dilp6 versus Actin-5c in heads and (e-f) $4 e-b p$ versus $\alpha$ tubulin in bodies of w1118 and dilp2-3,5 mutant flies. In the line plots letters indicate a significant difference between the diet levels and genotypes (lmer, $p<0.05)$.

\section{IIS and DR: shared and independent mechanisms}

Further downstream of IIS, expression of $4 e-b p$ in bodies of control flies was increased with lower yeast, consistent with previous found results (Zid et al. 2009). Upon dilp2-3,5 knockout, we measured unchanged expression pattern in responses to diet (Figure 2). This is in line with the notion that dilp6, possibly with components from other nutrient sensing pathways interconnected with $4 e-b p$, compensates for the loss of dilps to maintain the possibility of mediating the downstream effects of diet variation. But curiously, relative expression of $4 e$ - $b p$ was lower in bodies of the dilp mutants compared to controls, a situation that should correspond to elevated systemic insulin signaling, which is the opposite of what we expect for our mutants, but could indicate overcompensation in the mutants for the loss of dilp2, -3 and -5 within IIS or by other interconnected pathways (Partridge et al. 2010; Zoncu et al. 2011, figure 3). Nevertheless, the changed expression of dilp6 in response to diet preserves diet responsiveness upstream of IIS and may combine with other signaling outputs maintain downstream transcriptional circuits that are responsible for the response to the yeast dimension, but possibly not completely for the sugar 
dimension as the triple knockout reduces its lifespan response to the highest sugar levels (Zandveld et al. 2017, Figure 3). Expression patterns of the separate dilps do not only show a spatio-temporal pattern (Slaidina et al. 2009; Okamoto et al. 2009), but also a strong diet-dependency (Figure 1). This suggests that different dilps may have evolved through differences in functionality. Also among other gene families compensatory action is known, and are considered to co-exist for a better fine-tuning of downstream activities (Kafri et al. 2009). But in order to examine this further it would be interesting to test lifespan and reproduction effects upon dilp6 overexpression in a dilp mutant background. Another option would be assessing the diet responsiveness of a quadruple knockout of dilp2-3,5,6 but unfortunately these flies are not viable (Gronke et al. 2010). Altogether, this shows that dilps is essential in fruit flies, but also have a mutual redundancy from which we showed is diet-dependent. This shows dilp compensatory expression can maintain diet responsiveness upstream of insulin signaling, but cannot prevent the effects of decreased reproduction and lifespan extension by reduced insulin activity.

\section{CONCLUSION}

We have shown that upon deletion of dilp2-3,5, dilp6 expression was changed and became responsive to higher yeast levels. This preservation of dilp activity could not prevent the flies to drastically change their lifespan, but indicates a certain level of redundancy among dilps to maintain upstream nutrient responses. This is therefore the first study to show a diet-dependent compensatory action for the loss of dilps, proposing a potential mechanisms for the maintenance of nutrient homeostasis in the long-lived flies for certain diet dimensions. 


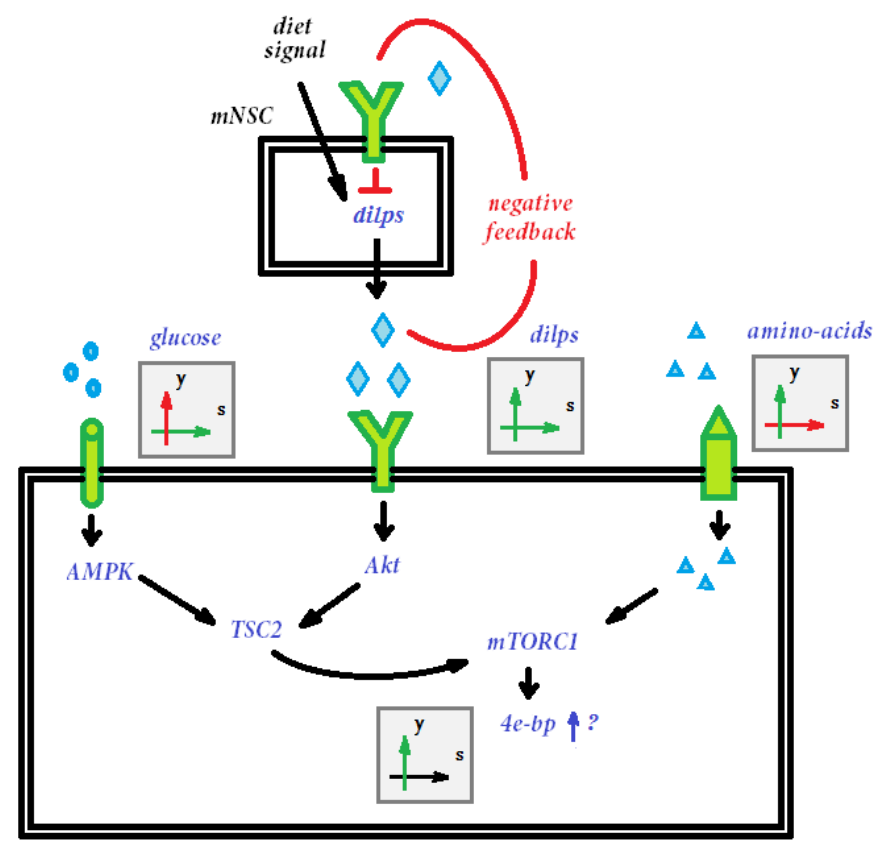

Figure 3: Suggested schematic overview of nutrient responses in Drosophila. Upon sugar and yeast sensing, the medio-neurosecretory cells (mNSC) in the fly brain, release insulin-like peptides in the haemolymph and a negative feedback loop maintains dilp activity upon lower DILP activity. This, together with other nutrient-sensing pathways, could maintain nutrient-homeostasis for the yeast-axis, but cannot compensate fully for lower insulin activity resulting in general lower $4 e-b p$ expression through lowered DILP activity. 


\section{Conflict of interest}

The authors do not have conflicts of interest to declare.

\section{Acknowledgments}

We acknowledge Vicencio Oostra for his advice on qPCR normalization and analysis, Mingyao Yang, Sahar Emran and Xiaoli He for their help in maintaining the lifespans and fly rearing and we want to thank all members of the Partridge and Gems laboratory and WUR Genetics department for all fruitful discussions.

\section{Author contributions}

M.D.W.P and B.J.Z designed the experiments. J.Z., J.H., B.J.Z. and M.D.W.P. wrote the manuscript. J.Z. and J.H. performed statistical analysis. J.Z. performed the experiments.

\section{Funding}

Work was funded by the Biotechnology and Biological Sciences Research Council (BB/I011544/1; M.D.W.P), the Royal Society (UF100158 \& RG110303; M.D.W.P), by the Network of Excellence LifeSpan (FP6/036894; J.Z., J.H., B.J.Z), and by the European Union's Seventh Framework Program (FP7/2007-2011) under grant agreement number 259679, IDEAL, J.Z., J.H., B.J.Z). 


\section{SUPPLEMENTAL MATERIAL}

\section{Figure $S 1$}

Response surface control flies and dilp2-3,5 mutants

\section{Figure S2}

Correlation dilp expression and lifespan/reproduction in control flies

\section{Figure S3}

Response surface relative expression dilp6 in bodies of controls and dilp2-3,5 mutants

\section{Table S1}

Primer sequences

\section{Table S2}

Data table gene expression values per diet and genotype

\section{Table S3}

Statistical analyses 


\section{Figure S1}

Response surface control flies and dilp2-3,5 mutants
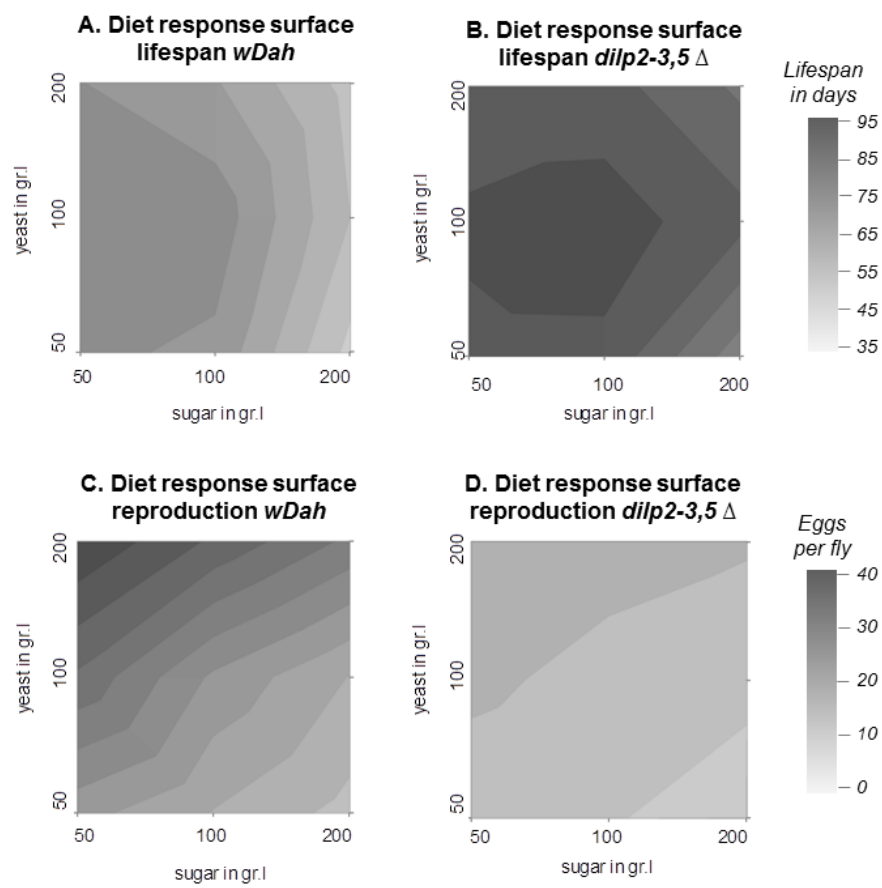

Figure S1: Previously reported lifespan response to dietary sugar and yeast for (A) control flies and (B) dilp23,5 mutants (from: Zandveld et al. (2017)). (C,D) Diet response surfaces for the eggs produced per fly on the $24 \mathrm{hrs}$ before sacrifice on day 7 for (C) control flies and (D) dilp2-3,5 mutants. 


\section{Figure S2}

\section{Correlation dilp expression and lifespan/reproduction in control flies}

Figure S2: Correlations between dilp expression in heads of control flies versus their lifespan and reproduction response

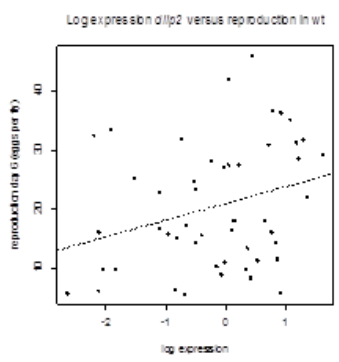

Multiple R-squared: 0.08398 p-value: 0.04341

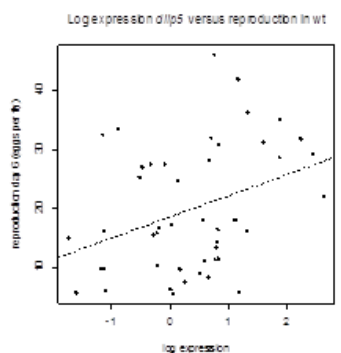

Multiple R-squared: 0.1233 p-value: 0.01671

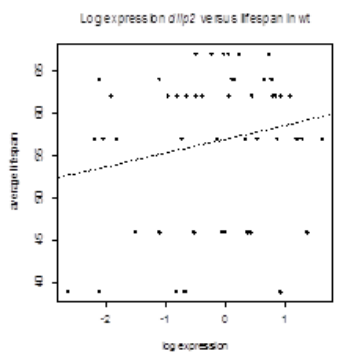

Multiple R-squared: 0.0391 p-value: 0.1729

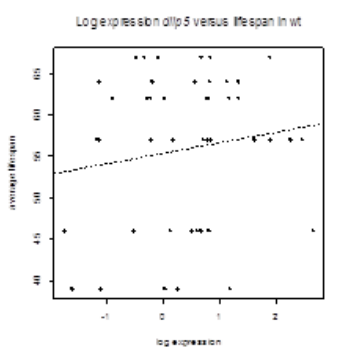

Multiple R-squared: 0.02048 p-value: 0.3427

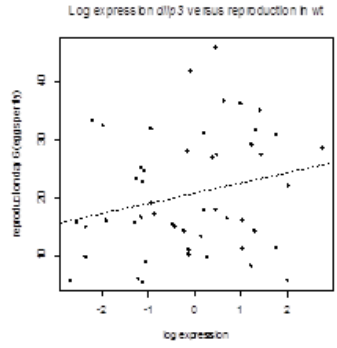

Multiple R-squared: 0.05595 p-value: 0.0947

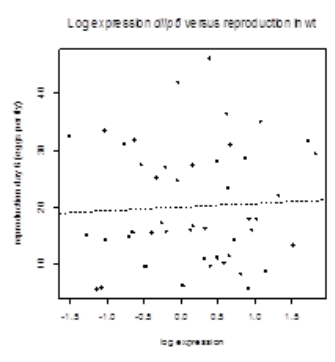

Multiple R-squared:0.00273 p-value: 0.7243

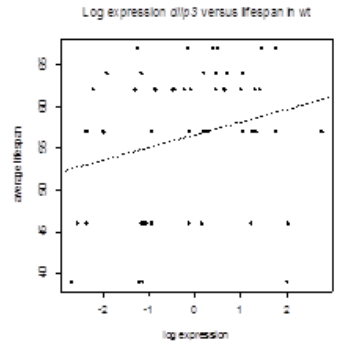

Multiple R-squared: 0.0585 p-value: 0.08727

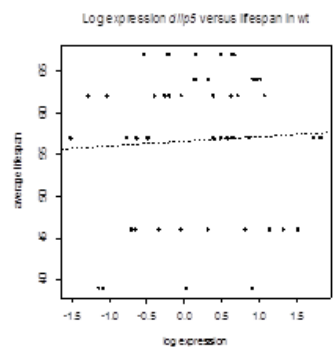

Multiple R-squared: 0.002777 p-value: 0.7221

Figure S2: Correlation between head dilp2,-3,-5 and -6 log relative expression and reproduction or lifespan for wild type flies. For every sample, reproduction is measured at the moment of sampling (day 6 on food treatment). For lifespan correlations, for every sample the average lifespan of that treatment cohort is used. Correlations are tested using a linear model, multiple R-squares and p-values are provided below each graph. 


\section{Figure S3}

Response surface relative expression dilp6 in bodies of controls and dilp2-3,5 mutants
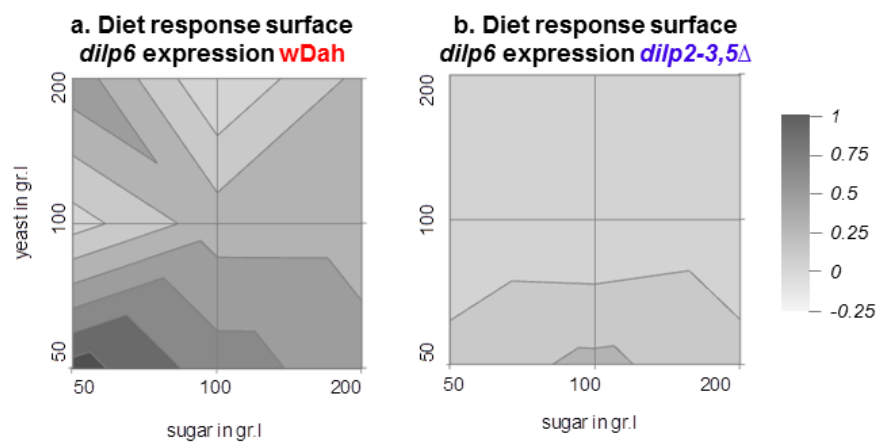

Figure S3: Diet response surfaces for relative expression of dilp6 in bodies of (a) control flies and (b) dilp23,5 mutants. In dilp2-3,5 mutants an increase with lower yeast levels is significant (lmer, $p<0.01)$, for $w D a h$ flies this effect seems present, but was not significant (lmer, $p<0.1)$. 


\section{Table S1.}

\section{Primer sequences}

Table S1: Primer sequences for dilp2, dilp3, dilp5, dilp6, 4e-bp, Act-5c and $\alpha$-tubulin

\begin{tabular}{|c|c|c|}
\hline Primer & & Sequence (5'-3') \\
\hline & Fw & ATGGTGTGCGAGGAGTATAATCC \\
\hline & $\mathrm{Rv}$ & TCGGCACCGGGCATG \\
\hline \multirow[t]{2}{*}{ dilp3 } & Fw & AGAGAACTTTGGACCCCGTGAA \\
\hline & $\mathrm{Rv}$ & TGAACCGAACTATCACTCAACAGTCT \\
\hline \multirow[t]{2}{*}{ dilp5 } & Fw & GAGGCACCTTGGGCCTATTC \\
\hline & $\mathrm{Rv}$ & CATGTGGTGAGATTCGGAGCTA \\
\hline \multirow[t]{2}{*}{ dilp6 } & $\mathrm{Fw}$ & CGATGTATTTCCCAACAGTTTCG \\
\hline & $\mathrm{Rv}$ & AAATCGGTTACGTTCTGCAAGTC \\
\hline \multirow[t]{2}{*}{$4 e-b p$} & $\mathrm{Fw}$ & CACTCCTGGAGGCACCA \\
\hline & $\mathrm{Rv}$ & GAGTTCCCCTCAGCAAGCAA \\
\hline \multirow[t]{2}{*}{ Act $-5 c$} & $\mathrm{Fw}$ & CACACCAAATCTTACAAAATGTGTGA \\
\hline & $\mathrm{Rv}$ & AATCCGGCCTTGCACATG \\
\hline \multirow[t]{2}{*}{$\alpha$-tubulin } & $\mathrm{Fw}$ & TGTCGCGTGTGAAACACTTC \\
\hline & $\mathrm{Rv}$ & AGCAGGCGTTTCCAATCTG \\
\hline
\end{tabular}




\section{Table S2}

\section{Data table gene expression values per diet and genotype}

Table S2: Per food type mean, median, standard error (se) and number of observations ( $\mathrm{n}$ ) for gene

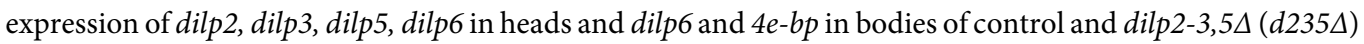
flies.

\begin{tabular}{|c|c|c|c|c|c|c|c|c|c|c|c|c|c|c|}
\hline & & \multirow{2}{*}{$\begin{array}{c}\text { yeast } \\
g . f^{-1}\end{array}$} & \multicolumn{4}{|c|}{$50 \mathrm{~g} \cdot \mathrm{l}^{-1}$ sugar } & \multicolumn{4}{|c|}{$100 \mathrm{~g} \cdot \mathrm{l}^{-1}$ sugar } & \multicolumn{4}{|c|}{$200 \mathrm{~g} . \mathrm{l}^{-1}$ sugar } \\
\hline & & & mean & median & se & $n$ & mean & median & se & $n$ & mean & median & se & $n$ \\
\hline \multirow{15}{*}{ 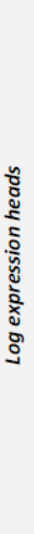 } & dilp2 & 50 & -0.18 & -0.25 & 0.11 & 6 & -0.17 & 0.04 & 0.22 & 6 & -0.47 & -0.36 & 0.27 & 5 \\
\hline & control & 100 & 0.02 & 0.00 & 0.07 & 6 & -0.11 & 0.05 & 0.20 & 6 & 0.07 & 0.07 & 0.05 & 4 \\
\hline & & 200 & 0.10 & 0.27 & 0.20 & 6 & 0.17 & 0.52 & 0.27 & 6 & -0.20 & -0.35 & 0.28 & 4 \\
\hline & dilp3 & 50 & -0.15 & -0.20 & 0.16 & 6 & -0.13 & 0.03 & 0.31 & 6 & -0.33 & -0.51 & 0.43 & 4 \\
\hline & control & 100 & 0.44 & 0.19 & 0.19 & 6 & -0.05 & 0.14 & 0.21 & 6 & -0.34 & -0.26 & 0.26 & 6 \\
\hline & & 200 & 0.30 & 0.24 & 0.23 & 6 & 0.19 & 0.31 & 0.31 & 6 & -0.20 & -0.48 & 0.27 & 5 \\
\hline & dilp5 & 50 & 0.03 & -0.04 & 0.11 & 4 & -0.06 & -0.01 & 0.16 & 6 & -0.09 & 0.01 & 0.18 & 6 \\
\hline & control & 100 & 0.05 & -0.04 & 0.11 & 5 & 0.18 & 0.30 & 0.16 & 6 & 0.07 & 0.25 & 0.21 & 5 \\
\hline & & 200 & 0.37 & 0.50 & 0.20 & 5 & 0.56 & 0.75 & 0.24 & 6 & 0.32 & 0.06 & 0.42 & 3 \\
\hline & dilp6 & 50 & -0.18 & -0.14 & 0.13 & 6 & 0.08 & 0.19 & 0.09 & 6 & -0.14 & -0.23 & 0.21 & 4 \\
\hline & control & 100 & 0.09 & 0.14 & 0.09 & 6 & 0.26 & 0.27 & 0.08 & 6 & 0.18 & 0.24 & 0.16 & 6 \\
\hline & & 200 & 0.09 & 0.17 & 0.16 & 5 & 0.11 & 0.05 & 0.25 & 6 & 0.14 & -0.02 & 0.22 & 3 \\
\hline & dilp6 & 50 & 0.04 & 0.03 & 0.09 & 6 & 0.26 & 0.26 & 0.11 & 6 & 0.09 & 0.03 & 0.12 & 4 \\
\hline & $d 235 \Delta$ & 100 & 0.11 & 0.03 & 0.11 & 6 & 0.04 & 0.26 & 0.28 & 5 & 0.27 & 0.26 & 0.09 & 5 \\
\hline & & 200 & 0.32 & 0.32 & 0.08 & 5 & 0.37 & 0.33 & 0.07 & 5 & 0.39 & 0.42 & 0.06 & 5 \\
\hline \multirow{12}{*}{ 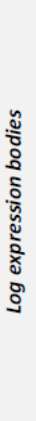 } & dilp6 & 50 & 0.87 & 0.84 & 0.07 & 4 & 0.58 & 0.42 & 0.28 & 5 & 0.41 & 0.52 & 0.16 & 4 \\
\hline & control & 100 & -0.02 & 0.13 & 0.35 & 4 & 0.28 & 0.28 & 0.26 & 2 & 0.28 & -0.01 & 0.21 & 5 \\
\hline & & 200 & 0.25 & 0.07 & 0.22 & 6 & -0.14 & -0.03 & 0.44 & 3 & 0.24 & 0.22 & 0.11 & 3 \\
\hline & dilp6 & 50 & 0.11 & 0.03 & 0.11 & 5 & 0.24 & 0.22 & 0.10 & 4 & 0.10 & 0.00 & 0.11 & 6 \\
\hline & $d 235 \Delta$ & 100 & -0.09 & -0.04 & 0.10 & 5 & -0.13 & -0.11 & 0.12 & 5 & -0.05 & -0.11 & 0.11 & 6 \\
\hline & & 200 & -0.15 & -0.29 & 0.16 & 5 & -0.13 & -0.13 & 0.08 & 5 & -0.04 & -0.12 & 0.06 & 6 \\
\hline & $4 e-b p$ & 50 & 0.54 & 0.54 & 0.15 & 6 & 0.63 & 0.52 & 0.24 & 4 & 0.43 & 0.55 & 0.15 & 5 \\
\hline & control & 100 & 0.23 & 0.23 & 0.09 & 6 & 0.51 & 0.40 & 0.19 & 5 & 0.47 & 0.50 & 0.11 & 5 \\
\hline & & 200 & 0.30 & 0.30 & 0.24 & 6 & 0.01 & -0.08 & 0.08 & 5 & 0.32 & 0.43 & 0.11 & 5 \\
\hline & $4 e-b p$ & 50 & 0.15 & 0.05 & 0.13 & 5 & 0.11 & 0.13 & 0.11 & 4 & 0.10 & 0.08 & 0.06 & 6 \\
\hline & $d 235 \Delta$ & 100 & -0.00 & 0.03 & 0.15 & 5 & -0.00 & 0.01 & 0.10 & 5 & -0.05 & -0.05 & 0.04 & 6 \\
\hline & & 200 & -0.09 & -0.04 & 0.09 & 5 & -0.03 & -0.00 & 0.06 & 5 & 0.04 & 0.03 & 0.06 & 6 \\
\hline
\end{tabular}




\section{Table S3}

\section{Statistical analyses}

Table S2.4: Linear mixed effects model parameter estimates for effects dietary sucrose (S), dietary (Y) yeast on normalized gene expression of dilp2, dilp3, dilp5 and dilp6 in heads and dilp6 in bodies of wild type and dilp2-3,5 mutants. No interaction between $\mathrm{S}$ and $\mathrm{Y}$ were found for dilps ( $\mathrm{p}>0.1$ ), so only single diet effects are shown. For $4 e-b p$ the interaction between genotype (gen), sucrose and yeast was determined. $\mathrm{z}$-value and $\mathrm{p}$ values are given. Significance levels: ‘’ $p<0.1 ;{ }^{(*)} p<0.05$; $^{\text {(*) }} \mathrm{p}<0.01$; ${ }^{(* *)} \mathrm{p}<0.001$

\begin{tabular}{|c|c|c|c|c|c|}
\hline Tissue/genotype & Gene & Factor & $z$-value & $\operatorname{Pr}>|t|$ & \\
\hline \multirow[t]{8}{*}{ heads $w D a h$} & dilp2 & S & -2.836 & 0.007 & ** \\
\hline & & $\mathrm{Y}$ & 2.817 & 0.007 & ** \\
\hline & dilp3 & S & -2.845 & 0.007 & ** \\
\hline & & $\mathrm{Y}$ & 2.030 & 0.04 & * \\
\hline & dilp5 & S & -1.089 & 0.283 & \\
\hline & & $\mathrm{Y}$ & 4.388 & 0.000 & $* * *$ \\
\hline & dilp6 & S & 1.567 & 0.125 & \\
\hline & & $\mathrm{Y}$ & 2.429 & 0.020 & * \\
\hline \multirow[t]{2}{*}{ heads dilp2-3,5 mutants } & dilp6 & $S$ & 0.772 & 0.445 & \\
\hline & & $\mathrm{Y}$ & 2.561 & 0.014 & * \\
\hline \multirow[t]{2}{*}{ bodies $w D a h$} & dilp6 & S & -0.396 & 0.695 & \\
\hline & & $\mathrm{Y}$ & -1.882 & 0.069 & . \\
\hline \multirow[t]{2}{*}{ bodies dilp2-3,5 mutants } & dilp6 & S & 0.343 & 0.733 & \\
\hline & & $\mathrm{Y}$ & -2.515 & 0.016 & * \\
\hline \multirow[t]{6}{*}{ bodies } & $4 e-b p$ & S & -0.116 & 0.908 & \\
\hline & & $\mathrm{Y}$ & -2.759 & 0.007 & ** \\
\hline & & gen & -3.108 & 0.003 & $* *$ \\
\hline & & $S^{*} Y$ & 1.164 & 0.248 & \\
\hline & & gen $* S$ & -0.404 & 0.688 & \\
\hline & & gen ${ }^{\star} Y$ & 1.373 & 0.174 & \\
\hline
\end{tabular}




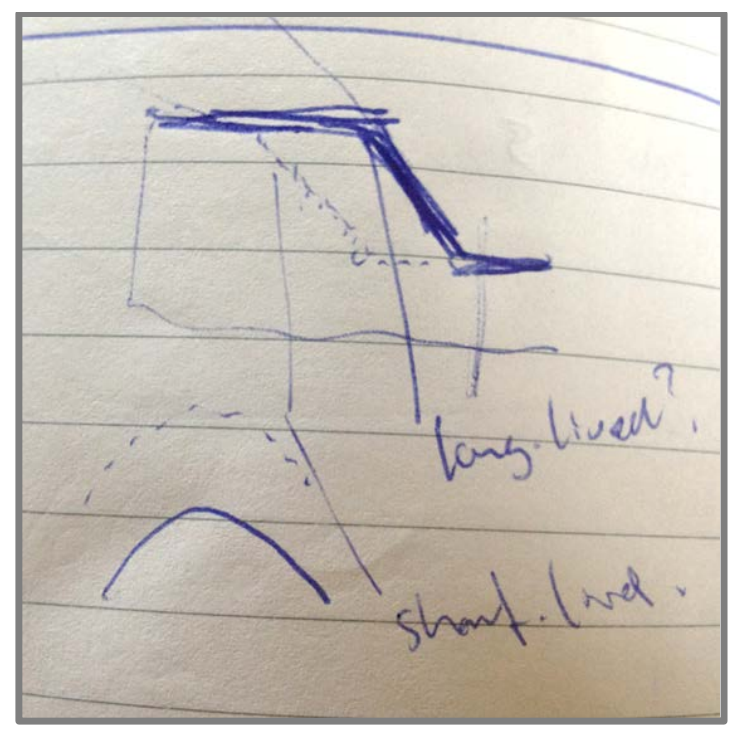

Linking mechanisms of general lifespan with the lifespan response to diet 


\title{
CHAPTER IV
}

\author{
NATURAL GENETIC VARIATION IN THE RESPONSE TO \\ NUTRITION IN THE FUNGUS PODOSPORA ANSERINA REVEALS \\ THAT LIFESPAN AND REPRODUCTION CAN BE UNCOUPLED
}

Jelle Zandveld

Suzette de Groot

Joost van den Heuvel

Fons Debets

Bas Johannes Zwaan 


\section{ABSTRACT}

Dietary restriction (DR), defined as undernutrition without malnutrition, increases lifespan in a variety of species including mammals, worms, fruit flies, and fungi. However, how DR extends lifespan is not well understood. In Podospora anserina, one of the few ageing fungi, DR also increases lifespan and is typically accompanied with a reduced pigmentation and decreased reproduction. By applying a broad range of glucose concentrations we constructed reaction norms for 62 natural occurring strains. We uncovered considerable natural variation in the shape of the reaction norms, including the glucose concentration at which lifespan increased (threshold $T$ ) and slope $S$ of the fungus' lifespan response to diet. Moreover, we identified a significant correlation between a strain's general lifespan and both parameters, suggesting that the lifespan response to diet partly acts through a mechanism involved in the fungus' general lifespan determination. Furthermore, for intermediate diets we demonstrated that reduced reproduction is not always associated with lifespan extension, indicating that decoupling of these traits that often show a trade-off can be achieved. We discuss the potential of studying natural variation in Podospora anserina for the identification of common genetic mechanisms mediating the lifespan response to diet and its consequences for other species, including humans. 


\section{INTRODUCTION}

Ageing, defined as the progressive loss of function accompanied by decreasing fertility and increasing mortality with advancing age (Kirkwood \& Austad 2000), is affected by both genetic as well as environmental factors. A well-studied phenomenon in ageing research is the effect of dietary restriction (DR), often defined as a reduction of food intake without malnutrition, which increases lifespan and improves health in many organisms (Weindruch \& Walford 1988; Fontana \& Partridge 2015). After the effect of DR was shown in rats (McCay et al. 1935) since then, more experiments were performed and showed similar effects for many organisms in a wide range of taxa, including fungi, fruit flies, spiders, rats, dogs, and non-human primates (reviewed by: Fontana \& Partridge 2015).

In addition to effects on lifespan, DR is often accompanied by other physiological effects, such as a differential response to environmental stressors in flies (Emran et al. 2014), reduced growth in fish (Terzibasi et al. 2009), faster mycelial growth in fungi (Van Diepeningen et al. 2010a). Notably, often a generally observed response in many organisms is a decreased reproductive output (Flatt 2011). Although these phenotypic effects of DR are well known, the mechanisms how DR causes them and how the trait responses are interrelated are not well understood.

Genetic knockouts, RNAi, recombinant inbred lines, and gene overexpression are a variety of genetic tools often used in the identification of the mediating mechanisms of lifespan extension by DR (Tatar et al. 2003; Bauer et al. 2010; Liao et al. 2010; Schleit et al. 2013; Stastna et al. 2015, Zandveld et al. 2017). To test whether a genetic change alters the lifespan response to diet, a genotype-by-environment interaction $\left(\mathrm{G}^{\star} \mathrm{E}\right.$ interaction) can be established. This interaction is present when the shape of the lifespan response to diet differs between genotypes. When genotypes have an identical or parallel lifespan response to diet change however, these genotypes are not a source of genetic variation for the DR response.

By using this framework, both within and between species natural genetic variation on the effects of DR is observed previously. For example, natural strains of C. elegans and C. remanei showed variation in their diet response; both an increased and decreased lifespan upon DR was observed (Sutphin \& Kaeberlein 2008; Stastna et al. 2015). Although no lifespan benefits in a wild mice strain was observed (Harper et al. 2006), a wild-derived strain of the short-lived fish N. furzeri showed a significant lifespan increase upon DR (Terzibazi et al. 2009). Also results from a DR meta-analysis across different species (Nagakawa et al. 2007) showed there is much inter-specific variation for the DR response. Importantly, the absence of a lifespan increase in some of these cases suggests that there is a large genotype or species-dependency for DR to increase lifespan.

New insights in ageing research however, suggest that multiple diets should be applied to state whether or not DR extends lifespan, because too few and suboptimal food treatments could easily 
conceal the effects of DR (Mair et al. 2008; Flatt 2014). Recently, several populations of wildderived Drosophila melanogaster were tested on a gradient of five food types. Although the response to lifespan differed between the natural populations, DR extended lifespan for all strains (Metaxakis \& Partridge, 2013). This genotype-independent lifespan increase upon DR would likely not have been observed if these strains had been tested on fewer diets. This indicates that if a perceived absence of a DR response, like previously reported (Sutphin \& Kaeberlein 2008; Stastna et al. 2015; Harper et al. 2006; Nagakawa et al. 2012), may very well be present but be unveiled only by applying multiple diets (Mair \& Dillin 2008; Flatt 2014).

But using a broad range of diets is also helpful for a more detailed analysis of the $\mathrm{G}^{\star} \mathrm{E}$ response to diet. Namely, is possible to separate two important response parameters, i.e. the threshold (or onset) and the slope of the response. Namely, when genotypes differ in the threshold of the response, the lifespan pattern is unchanged but moved along the dietary axis. Genotypes that differ in the slope (or incline) of the lifespan response show a different lifespan pattern upon diet change. On too few diets, whether a $\mathrm{G}^{\star} \mathrm{E}$ interaction is a consequence of a changed threshold or slope cannot be identified, only by testing multiple diets this is possible (Mair \& Dillin 2008; Flatt 2014). Furthermore, also when assessing food types with large nutritional differences, this will likely result in co-varying traits between the treatments. This makes it difficult to disentangle diet effects on different life-history traits (Flatt 2014). For example, in many organisms lifespan and reproduction show often a trade-off upon DR; reproduction often decreases while lifespan increases (Fontana \& Partridge, 2010). But it is possible to negate this trade-off with small and specific dietary modifications (Grandison et al. 2009; Piper et al. 2017). So instead of using a few nutritionally distinct diets, assessing ageing-related phenotypes on a gradual diet (i.e. using small nutritional steps between treatments) will more likely result in a reproduction pattern that differs from the lifespan pattern. If these phenotypic patterns indeed vary, this allows to parse apart the diet effects on lifespan and reproduction.

P. anserina, one of the few ageing filamentous ascomycete fungi, has a strain-dependent short lifespan, a known ecology, a sequenced genome, the ability to grow on a synthetic diet, and has been subject of extensive past work on the mitochondrial mechanisms of ageing. This altogether, marks Podospora as a useful organism to study the genetics of lifespan (reviewed by: Osiewacz 2002). Also Podospora extends lifespan upon DR, but in contrast to other organisms, in Podospora the lifespan increase appears to be very abrupt and extremely plastic, showing a potentially indefinite lifespan increase upon DR (Maas et al. 2004). The seemingly on-off strategy to DR - i.e. increased lifespan, increased growth rate, repressed fertility (i.e. perithecia formation) and fewer pigmentation (Maas et al. 2004) - makes Podospora an interesting and unique organism to study this plastic response. Moreover, DR in many organisms is applied through dilution of multiple diet components (Piper \& Partridge 2007, but see Piper et al. 2014), the P. anserina synthetic medium (PASM) allows for DR to be applied through glucose or nitrogen restriction only (Van 
Diepeningen et al. 2010a; Van Diepeningen et al. 2014) potentially enabling the more direct identification of cause and effect.

Previous experiments on the effects of DR in natural strains of Podospora (Maas et al. 2004; Maas et al. 2007; Van Diepeningen et al. 2010a, Van Diepeningen et al. 2010b, Van Diepeningen et al. 2014) showed that wild-derived strains differ substantially in their glucose sensitivity; effects have been observed ranging from no response to a response of $500 \%$ and even $1000 \%$ lifespan extension on the highest level of glucose restriction (Maas et al. 2004; Van Diepeningen et al. 2010a, Van Diepeningen et al. 2010b). Furthermore, natural isolates were found to be polymorphic for the presence of the pAL2-1 senescence plasmid (Maas et al. 2004) that negates the DR effect as a mitochondrial mutator (Maas et al. 2007). Strains that contain the $p A L 2-1$ plasmid were found to have a reduced lifespan increase upon glucose restriction; an effect verified by removing the $p A L 2$ 1 plasmid from a strain that naturally possesses the plasmid (Van Diepeningen et al. 2008). By taking advantage of these unique traits of Podospora anserina, investigating natural strains holds the promise of shedding light on the genetic mechanism of the lifespan response to diet, knowledge that when linked to other species may facilitate the identification of common mechanisms of beneficial DR effects.

As the results from previous Podospora research were acquired by investigating the strains on three diets, more insight in the fungus' response to glucose could be obtained by investigating strains on a more gradually changing diet. Through applying a broad and continuous glucose gradient, we indeed obtained a more detailed response for lifespan, pigmentation and reproduction from over 60 natural isolates of Podospora. This data enabled us to identify natural genetic variation in both the threshold and the slope of the lifespan response to diet and in addition, to test whether typical lifespan-co-varying phenotypes are always coupled with the pattern of lifespan upon diet change.

\section{EXPERIMENTAL PROCEDURES}

\section{Strains and culturing conditions}

Fungal samples have been collected between 1991 and 2001 in Wageningen and Arnhem, The Netherlands (Van Diepeningen et al. 2008), and spores have been stored in the freezer at $-80^{\circ} \mathrm{C}$. Strains that contain the pAL2-1 senescence plasmid were excluded since this plasmid interferes with the DR effect (Van Diepeningen et al. 2010a), leaving 63 strains available for testing (Supplement 1).

The $P$. anserina synthetic medium (PASM) (described by Esser 1974) contains per liter $20 \mathrm{~g}$ agar, $1 \mathrm{~g}$ urea, $0.5 \mathrm{~g} \mathrm{KH} 2 \mathrm{PO} 4,0.6 \mathrm{~g} \mathrm{~K} 2 \mathrm{HPO} 4,0.5 \mathrm{~g} \mathrm{MgSO}$, biotin and thiamin were added to a final concentration of $100 \mathrm{~g} / \mathrm{mL}$ as well as $1 \mathrm{~mL}$ trace element solution per liter. Our trace element solution contained per $100 \mathrm{~mL} 500 \mathrm{mg}$ citric acid.H2O, $500 \mathrm{mg} \mathrm{ZnSO} 4.7 \mathrm{H} 2 \mathrm{O}, 100 \mathrm{mg}$ $\mathrm{Fe}(\mathrm{NH} 4) 2 \mathrm{SO} 4.6 \mathrm{H} 2 \mathrm{O}, 25 \mathrm{mg} \mathrm{CuSO} 4.5 \mathrm{H} 2 \mathrm{O}, 5 \mathrm{mg} \mathrm{MsSO} 4,5 \mathrm{mg} \mathrm{H} 3 \mathrm{BO} 3$, and $5 \mathrm{mg} \mathrm{Na} 2 \mathrm{MoO} 4-$ $2 \mathrm{H} 2 \mathrm{O}$. As standard carbon source glucose was added, for the different treatments respectively 0.02 , 
$0.05,0.10,0.20,0.50,1.0$ and 2.0 grams per $100 \mathrm{ml}$. On all seven glucose concentrations each strain was tested in three-fold. Experiments were performed in a $28^{\circ} \mathrm{C}$ dark stove.

\section{Lifespan}

Spores of $P$. anserina isolated from the glycerol stock at $-80 \mathrm{C}$ were plated on $2 \%$ PASM and acetate $(3.9 \mathrm{~g} / \mathrm{l})$. Mycelia from these plates were used to inoculate $30 \mathrm{~cm}$ long 'race tubes' containing $20 \mathrm{ml}$ PASM with different glucose concentrations. For each strain lifespan was determined for triplicate inoculations, measured in mm (Maas et al. 2004; Van Diepeningen et al. 2010a; Osiewacz 2002). During the three months of the experiment, strain growth was monitored every three or four days and after three months total growth was determined. Colonies were considered to be 'alive' if after 90 days the strains were still growing or the end of the $30 \mathrm{~cm}$ tube was reached. Colonies were considered 'dead' if growth was ceased before reaching the end of the tube.

\section{Lifespan-associated phenotypes}

On high glucose levels, Podospora typically shows pigmentation, which increases in senescent cells (Osiewacz 2002) and decreases typically with glucose restriction (Van Diepeningen et al. 2010a). For each strain, replicate, and glucose concentration we scored the pigmentation phenotype as 'present', i.e. when any pigmentation was visible to the naked eye, or 'absent', i.e. when strains were solely white or transparent. On high glucose, Podospora typically shows an increase of perithecia formation whereas on low glucose concentrations perithecia are absent. For this reproduction phenotype of Podospora, we also applied a binominal categorization; only the presence or absence of perithecia was scored.

\section{Model parameters}

The diet response pattern of each strain was fitted to a non-linear model $(g n l m)$ were lifespan (L) was dependent on glucose concentration ([glucose $]$ ) using the equation below:

$$
\left.L=\frac{c 1}{1+\exp ^{(S *[\text { glucose }]-T))}}+c 2 \quad \text { (equation } 1\right)
$$

Parameters $S$ and $T$ estimate respectively the slope and threshold of lifespan in response to glucose concentration. The parameters $\mathrm{c} 1$ and $\mathrm{c} 2$ are implemented to increase the fit of the model, but are not affecting any of the two DR response parameters. For further explanation of the model see Figure 1.

\section{Statistical analysis}

To correlate the lifespan of strains to the model parameters $S$ and $T$, we applied a linear model $(\mathrm{lm})$ using $R$ ( $R$ Core Team 2012). To assess the association between lifespan and the lifespan-related phenotypes perithecia and pigmentation, we performed a chi-square test. These tests were thus performed separately: One for the association between survival and presence of pigmentation, and 
one for survival and presence of perithecia formation. Chi-square values were calculated for the entire dataset as well as stratified per glucose concentration. A significance level of $\mathrm{p}<0.05$ was used for a significant statistical association, which corresponds to a chi-square value of 3.84 .

a.

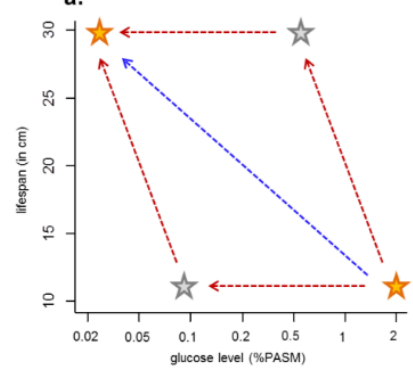

b.

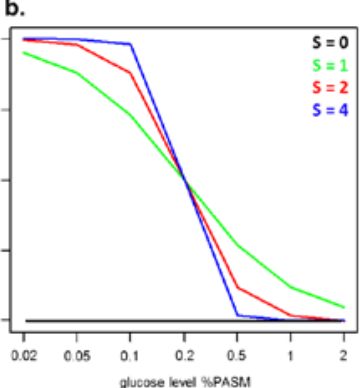

c.

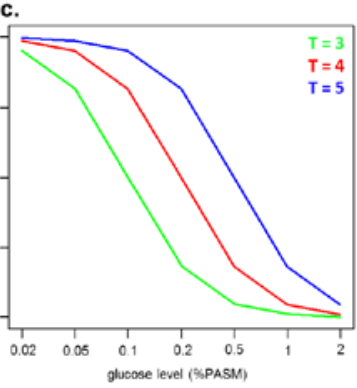

Figure 1: Lifespan response patterns to diet of Podospora (a) When measuring lifespan on a few and nutritionally distinct diets, an actual different responses between genotypes (two grey stars, red line) can be perceived as an identical response (two yellow stars, blue line). Genotypes with a perceived weaker slope (blue line) can in fact have a much stronger response, but differ in the onset of their response (red lines). (b-c) Therefore, by fitting a non-linear model (equation 1) to the lifespan response to seven diets for each strain measured in this study, two different parameters/features of the lifespan response were estimated. (b) The slope $S$ of the glucose response can vary from no response to glucose $(S=0)$ to a very strong response $(S \geq 4)$. (c) The threshold $T$ estimates the onset of the lifespan extension upon glucose restriction. If $T$ is high, a strain's lifespan starts to increase on relatively high glucose levels (e.g. $T=5$ ). So if $T$ is low, a strain requires a more severe restriction for a lifespan extension (e.g $T=3$ ). 
a. Variation slope $S$

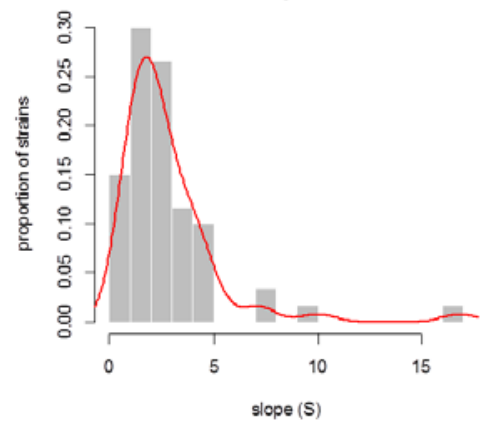

c. Different slope $S$

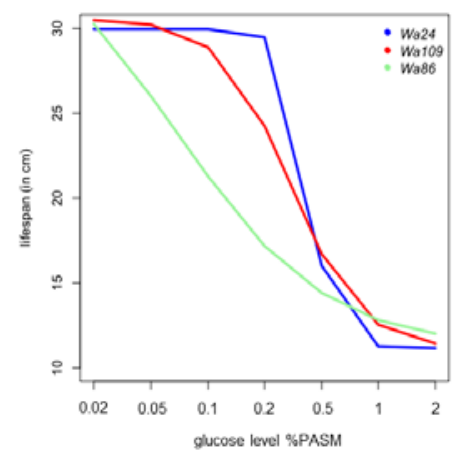

e. Lifespan vs slope $S$

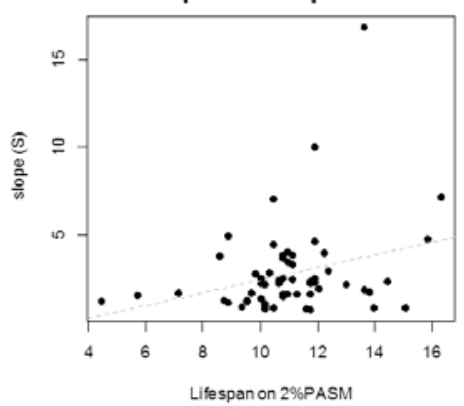

b. Variation threshold $T$

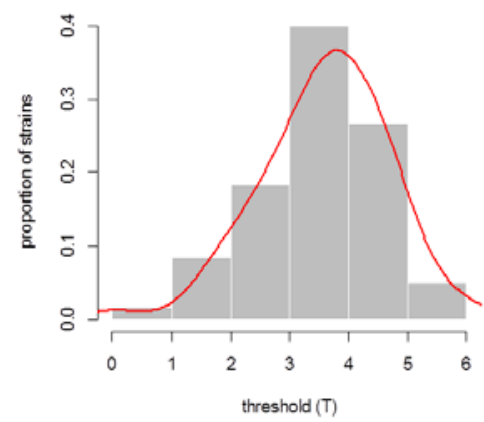

d. Different threshold $T$

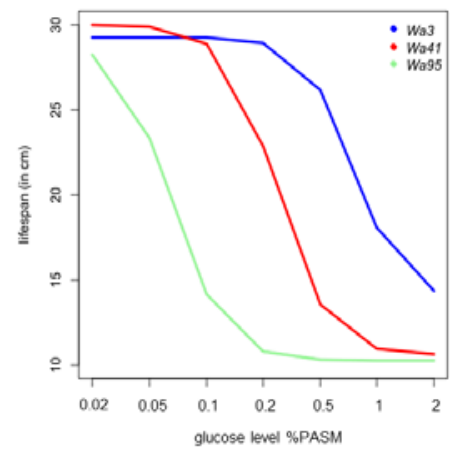

f. Lifespan vs threshold $T$

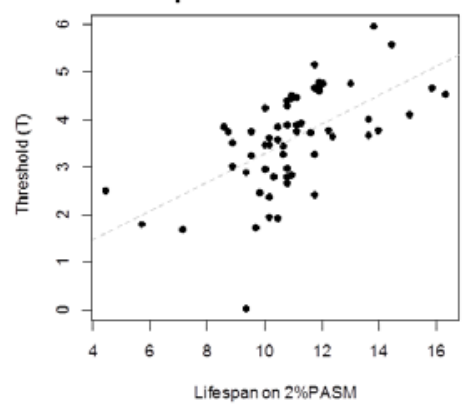

Figure 2: Natural variation for Podospora strains in their response to glucose. (a,b) The frequency distribution and fitted average (red line) of the variation in the slope $S$ (a) and the threshold $T$ (b) of lifespan responses to glucose. (c,d) Representative examples of natural strains that differ in $S$ and $T$ parameters. (e,f) Correlations and fitted line (linear model) between lifespan on 2\%PASM and the diet response parameters $S$ and $T$. 


\section{RESULTS}

Natural variation in response to glucose

To estimate the lifespan change in response to a range of glucose concentrations, we fitted a nonlinear model to the lifespan data of each of the 61 tested wild-derived strains (equation 1, Figure 1 ), and found substantial variation the estimated threshold and slope parameters, $T$ and $S$. One strain, Wa63, showed too much alterations between diets, and could not be fitted using the model. Therefore, our analysis is based on the variation between 60 wild- derived strains of Podospora anserina.

The slope ' $S$ ' of the response identifies whether strains show a lifespan increase with glucose restrictionWe found that all strains showed an increase of lifespan upon glucose restriction $(S>0$; Figure 2a). The nine strains for which the smallest response was detected, i.e. $S<1$, still all showed a lifespan increase upon glucose restriction $(g n l m, S>0, p<0.05)$. Although we measured an average response $S$ of $2.81 \pm 0.32$ (s.e.), more abrupt responses were also observed; four strains namely showed a response $S>7$ (Figure $2 \mathrm{a}$ ).

The threshold ' $T$ ' identifies the onset of the lifespan increase upon glucose restriction, and was shown to vary considerably between the strains (Figure $2 b$ ), with an average response $T$ of $3.59 \pm$ 0.14 (s.e.) and a range between 0.03 and 5.95 .

This large variability in the responses parameters allowed us to identify groups of strains with low and high response values $S$ and $\mathrm{T}$, but also strains with a similar threshold $T$ but different slope $S$, and vice versa. In strains that differ in $S$, lifespan on higher glucose concentrations can be similar but deviates with further glucose restriction, so the level of lifespan increase upon glucose restriction differs (Figure 2c). Similarly, two strains that differ in $T$, the pattern of lifespan change upon glucose restriction can be very similar but shifted along the glucose gradient (Figure $2 \mathrm{~d}$ ).

\section{Correlation between lifespan and the response to glucose}

By measuring the strains on a gradient of glucose concentrations allowed us to test for a relationship between general lifespan under ample nutritional conditions (2\% PASM )and the estimated DR lifespan response parameters $S$ and $T$. Interestingly, we found a correlation between lifespan on 2\% PASM and both parameters $S$ and $T$ (Figure 2e,f). Lifespan was slightly but significantly correlated with the slope $S$ of the lifespan response $\left(\operatorname{lm}, R^{2}=0.07, p<0.05\right)$. A tighter correlation was observed between lifespan and the threshold $T$ of the lifespan response $(\mathrm{lm}$, $\left.R^{2}=0.34, p<0.001\right)$.

\section{Association between survival and lifespan-related phenotypes}

To assess the association between different phenotypes that typically co-vary upon dietary interventions that extend lifespan, we recorded the presence of pigmentation and perithecia. When we pooled all data of every glucose level, we showed that a long-lived phenotype was associated with the absence of pigmentation and perithecia formation $\left(X^{2}, p<0.001\right)$ (Table 1, Table 2). When testing the association between lifespan and pigmentation within each glucose level, we observed 
a similar association between lifespan and presence of pigmentation $\left(X^{2}, p<0.001\right)$. With some exceptions, short-lived strains were highly associated with the presence of pigmentation $\left(X^{2}\right.$, $p<0.001)$, and longer-lived strains with the absence of pigmentation $\left(X^{2}, p<0.001\right)$ (Table 1$)$, irrespective of glucose level under consideration.

When we investigated the association between lifespan and perithecia formation, the pooled data also showed a significant association between lifespan and the presence of perithecia (Tabel 2). When testing this association per glucose level, however, we found no significant association between lifespan and presence of perithecia for $0.1 \%$ and $0.2 \% \operatorname{PASM}\left(X^{2}, p>0.1\right)$. This means that there are strains that differ in their lifespan response but show the same reproduction phenotype. For instance, in figure 3 two strains are shown that differ in their lifespan threshold but have a similar reproduction phenotype. That is, on $0.2 \%$ PASM Wa76 shows a typical response to diet (i.e. an absence of perithecia and long lifespan) but $W a 79$ is short-lived despite the absence of reproduction (Figure 3 ).

Table 1: The association between lifespan and pigmentation. For each of the four phenotypic combinations, the number of observations are indicated per glucose level, and summarized in the pie chart below each column. If chi-square values $\left(X^{2}\right)$ could be estimated, the $X^{2}$-values and their significance levels (indicated with stars) are provided per glucose level and for the pooled dataset.

\begin{tabular}{|c|c|c|c|c|c|c|c|c|c|}
\hline \multicolumn{2}{|c|}{ phenotypes } & \multicolumn{8}{|c|}{ Glucose concentration } \\
\hline short-lived & pigment & $0.02 \%$ & $0.05 \%$ & $0.1 \%$ & $0.2 \%$ & $0.5 \%$ & $1 \%$ & $2 \%$ & pooled \\
\hline 0 & 0 & 131 & 115 & 62 & 14 & 0 & 0 & 0 & 322 \\
\hline 0 & 1 & 0 & 1 & 1 & 3 & 2 & 0 & 0 & 7 \\
\hline 1 & 0 & 11 & 18 & 22 & 7 & 0 & 0 & 0 & 58 \\
\hline 1 & 1 & 7 & 15 & 65 & 126 & 148 & 149 & 150 & 660 \\
\hline & & $45.1^{\cdots}$ & $48.7^{\cdots}$ & $76.4^{\cdots \cdots}$ & $68.1^{\cdots}$ & - & - & - & $782.9^{\cdots \cdots}$ \\
\hline
\end{tabular}

Table 2: The association between lifespan and perithecia formation. For each of the four phenotypic combinations, the number of observations are indicated per glucose level, and summarized in the pie chart below each column. If chi-square values $\left(X^{2}\right)$ could be estimated, the $X^{2}$-values and their significance levels (indicated with stars) are provided per glucose level and for all data together (pooled).

\begin{tabular}{|c|c|c|c|c|c|c|c|c|c|}
\hline \multicolumn{2}{|c|}{ phenotypes } & \multicolumn{8}{|c|}{ Glucose concentration } \\
\hline short-lived & perithecia & $0.02 \%$ & $0.05 \%$ & $0.1 \%$ & $0.2 \%$ & $0.5 \%$ & $1 \%$ & $2 \%$ & pooled \\
\hline 0 & 0 & 131 & 116 & 61 & 15 & 0 & 0 & 0 & 323 \\
\hline 0 & 1 & 0 & 0 & 2 & 2 & 2 & 0 & 0 & 6 \\
\hline 1 & 0 & 18 & 33 & 86 & 106 & 1 & 1 & 0 & 245 \\
\hline \multirow[t]{2}{*}{1} & 1 & 0 & 0 & 1 & 27 & 147 & 148 & 150 & 473 \\
\hline & $X^{2}$ & - & - & 0.1 & 0.3 & $18.1^{* * *}$ & - & - & $370.4^{* * *}$ \\
\hline
\end{tabular}




\section{DISCUSSION}

In this study we characterized genotype-dependent lifespan response patterns to diet of over 60 wild-derived strains of $P$. anserina. By applying a broad range of glucose concentrations we aimed (1) to distinguish more detailed effects of the fungus' lifespan response to diet, such as the slope and the threshold of the lifespan response, and in addition, (2) to identify whether two typical lifespan-related traits, pigmentation and perithecia formation, consistently co-vary with the lifespan effects in response to dietary change.

To our knowledge, this is the first study applying a $\mathrm{G}^{\star} \mathrm{E}$ framework using a gradual changing diet on such an extensive set of natural genetic variants. By fitting a non-linear model to the lifespan data we estimated and determined substantial natural genetic variation in the two main response parameters determining the diet response of lifespan, namely the slope $S$ and the threshold $T$ of the diet response. All strains had an increased lifespan upon glucose restriction, but we showed considerable variation in the responses between strains. We also found a significant correlation between lifespan on 2\% PASM and both response parameters $S$ and $T$. Furthermore, pigmentation and perithecia formation, two typical lifespan-related traits, showed differential effects in their correlation with lifespan change (Table 1\&2): on intermediate glucose levels, the absence of perithecia was not significantly correlated to a long-lived phenotype, whereas pigmentation showed an association with lifespan. These findings stress the importance and possibilities of applying multiple diets when investigating the effects of dietary restriction.

The number of strains, the broad range of glucose concentrations, and the strong lifespan increase upon glucose restriction typically observed in Podospora, altogether enabled us to analyse the DR response in more detail. Maas et al. $(2004,2007)$ and Van Diepeningen et al. (2008) previously studied the response of natural strains of Podospora anserina, and also showed considerable genetic variation in the lifespan response to diet for different glucose levels, and found that the $p A L-2$ plasmid strongly interacts with the lifespan response to DR, abolishing the response to glucose deprivation in some strains (Maas et al. 2004; Van Diepeningen et al. 2008). By using natural strains that do not contain this plasmid and applying a much broader range of diets in this study, we improved our estimation of the slope $S$ and especially the threshold $T$ of DR responses in Podospora. 

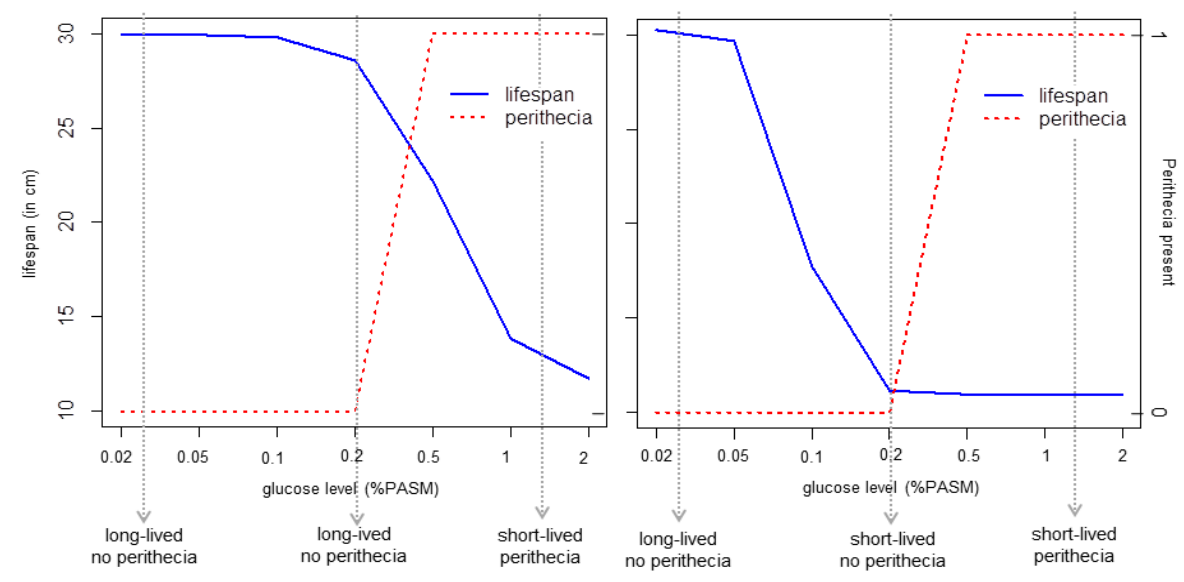

Figure 3: Two different co-variation patterns between lifespan and perithecia patterns upon diet change. (a) In strain Wa76 the lifespan pattern with glucose change shows a strong co-variation with perithecia formation, e.g. on $0.2 \%$ PASM the strain increased lifespan and also shows an absence of perithecia formation. (b) Another strain ( $W a 79$ ) is short-lived on $0.2 \%$ PASM, but, similar to Wa76, showed an absence of perithecia formation on this glucose level too.

\section{Lifespan effects of glucose restriction}

Although we observed considerable variation in both response parameters, all strains showed a lifespan increase under DR conditions, i.e. the estimated slope was for every strain higher than zero (Figure 2a,b). Some strains showed an increase of lifespan only upon severe glucose restriction, an effects that would not have been observed if the level of glucose restriction had been limited to higher concentrations. For example, the strain Wa95 appears not to respond to glucose restriction between $2 \%$ and $0.2 \%$ PASM, but does have an increased lifespan at glucose concentrations lower than $0.2 \%$ (Figure $2 \mathrm{f}$ ). This shows that obvious responsive strains are considered to be non-responders if lifespan is only determined on relatively high glucose levels. In line with this, the only other study applying multiple diets has similarly shown that natural populations differ, but always have a lifespan increase upon DR (Metaxakis \& Partridge, 2013). Interestingly, studies reporting on non-responders or a decreased lifespan upon DR, hardly applied more than two diets (Sutphin \& Kaeberlein 2008; Stastna et al. 2015; Harper et al. 2006; Nagakawa et al. 2012). Altogether, this suggests that a lifespan increase by diet can always be achieved under the right dietary conditions. Furthermore, new insights in nutritional geometry in other model systems showed that an increase in lifespan by dietary intervention is not only achieved by simply restricting energy, but rather by combinatory effects of different macronutrients (Piper et al. 2017; Solon-Biet et al. 2014; Simpson \& Raubenheimer 2012). Applying these newest insights to strains with weak responses to glucose in this study (e.g. Wa25 or Wa81, Table S1) may potentially reveal responsiveness to other nutrient-dimensions. 


\section{Natural genetic variation response parameters $S$ and $T$}

Previous studies have found variation in the lifespan response to dietary restriction too, but the limited number of diets used does not allow the analyses of response parameters similar to ours (Sutphin, Kaeberlein, 2008; Stastna et al. 2015). In contrast, the Drosophila study using a range of diets (Metaxakis \& Partridge, 2013) showed variation for the incline of the lifespan response to diet (i.e. the slope of the response), as well for the parameter most closely resembling the threshold in our study, the lifespan-by-diet optimum. Indeed, lifespan of the four populations peaked at different diets (Metaxakis, Partridge, 2013), which is in concordance with natural genetic variation for the threshold $T$ observed in our dataset (Figure 2).

Any intervention that decreases the slope of the lifespan response is reducing the interaction between diet and lifespan, for example by a disabled regulatory mechanisms of diet responses (reviewed by: Flatt, 2014; Mair \& Dillin 2008). The observed (small but significant) correlation between lifespan on 2\% PASM and the slope of the strains' lifespan response, suggests overlapping mechanisms between the general ageing process and the processes determining the fungus' response to diet. Because this relationship is positive, i.e. a relatively higher slope for strains that live longer, it suggests that the similar processes that benefits ageing would also partly regulate lifespan increases through diet. In line with this, our laboratory recently reported a link between ageing and the lifespan response to diet. Namely, shorter-lived strains with knockouts in autophagy pathways had a shorter period in which lifespan could be extended by DR (Van Diepeningen et al. 2014), and suggests that through autophagy deficiency damage accumulates faster and prevent further lifespan increase through DR.

Interestingly, mitochondria are associated with lifespan extension and effects of diet restriction not only in Podospora (Ociewacz 2011), but also other organisms, possibly including humans (Guarente 2008). In Podospora mitochondria of aged strains and ones subjected to 2\%PASM both show accumulated quantities of $\operatorname{sen} D N A-\alpha$, an intron highly associated with mtDNA stability (Van Diepeningen et al. 2010a). The previously discussed pAL2-1 plasmid, too, interferes with mitochondrial function and decreases lifespan and reduces Podospora diet responses by acting as a mitochondrial mutator (Maas et al. 2007). The processes that increase lifespan under DR conditions are therefore, similar to lifespan in general, regulated at least partly through mitochondrial quality.

A genotype that affects the threshold of the response possibly differs in the sensitivity to glucose. Strains that, for example, have a reduced nutrient intake or a disabled energy machinery, may experience an increased lifespan on relatively higher glucose levels. These genotypes shift the DR response pattern to higher glucose levels-but not necessarily by changing the shape of the lifespan pattern-and as a result, live longer on higher glucose levels. This pattern is demonstrated with our data in figure $2 \mathrm{~b}$, were strain $W a 3$ shows a lifespan change similar to those of strains $W a 41$ and Wa95, only on higher glucose levels (Figure 2f). This may explain the strong relationship observed between lifespan on 2\%PASM and the response parameter $T$ (Figure 2d). Likewise, this concept is 
also demonstrated in Drosophila mutants for chico, a gene encoding the insulin receptor substrate, were a perceived lifespan extension (Clancy et al. 2001) was actually a shifted lifespan response with diet (Clancy et al. 2002).

Implications of correlation between lifespan and lifespan-related phenotypes pigment and perithecia. Genotypic differences between the threshold of the DR response also provides new, unexplored possibilities in the study of the lifespan responses to diet. Namely, we showed that on intermediate glucose levels lifespan and perithecia formation were not significantly associated, i.e. a short lifespan is not necessarily accompanied by the presence of perithecia and vice versa. When studying the effects of diet on lifespan, for example by measuring gene expression, a decoupling of trait responses will identify separate effector genes for the responses of the different traits. We found different strains with differential effects of perithecia formation and lifespan change with diet. For example, when testing strains Wa76 and Wa79 on 0.2\%PASM (Figure 3) we observed Wa76 lived longer, but both strains reduced perithecia formation. Therefore, we may expect to find lifespan effector genes being upregulated by Wa76, but without the interfering effects of genes involved in reproduction often found in gene expression upon DR (Pletcher et al. 2002; Doroszuk et al. 2012; Chapter 7, this thesis). These findings will improve the extraction of genes to be more likely associated to only lifespan, and without interference of co-varying processes. The observed strong association between lifespan and pigmentation shows however, that decoupling of these traits is more difficult to establish and may suggest a tighter genetic connection between the pigment formation and lifespan response to diet than between reproduction and lifespan.

\section{CONCLUSION}

In conclusion, we have uncovered ample natural variation in the DR responses of Podospora anserina. Measuring genotype-by-diet interactions in a large set of natural isolates, we identified genetic variation for both the slope and threshold of the diet response, which suggests partly overlapping mechanisms determine the response of lifespan to glucose and a strain's general lifespan. These findings, and the observed atypical associations between reproduction and lifespan for some strains, show that glucose restriction in Podospora anserina is a promising system for further identification of lifespan effector genes of the fungus' DR response. 


\section{Competing interests}

All authors state not to have competing interests.

\section{Authors' contributions}

JZ wrote the manuscript, performed data analysis, carried out the statistical analyses and participated in the design of the study; SG carried out the laboratory work, participated in data analysis, participated in the design of the study and drafted the manuscript; JvdH participated in data analysis, participated in the statistical analyses and participated in writing the manuscript; FD coordinated the study, participated in the design of the study, and writing of the manuscript; BZ coordinated the study, participated in the design of the study and in writing the manuscript.

\section{Acknowledgements}

We acknowledge Marijke Slakhorst for their help in the preparation of the strains and preparing media. We thank all members of the Genetics laboratory (Wageningen University and Research) for their useful comments. We thank Anne van Diepeningen (CBS, Utrecht) for sharing ideas and useful comments.

\section{SUPPLEMENTAL MATERIAL}

\section{Table S1}

List and model parameters of all strains 


\section{Table S1}

List and model parameters of all strains

Table S1: List of all tested strains, average lifespan on each glucose level and model parameter estimations for $\mathrm{c} 1, \mathrm{~S}, \mathrm{~T}$ and $\mathrm{c} 2$ (equation 1).

\begin{tabular}{|c|c|c|c|c|c|c|c|c|c|c|c|}
\hline strain & $0.02 \%$ & $0.05 \%$ & $0.1 \%$ & $0.2 \%$ & $0.5 \%$ & $1 \%$ & $2 \%$ & $c 1$ & $S$ & $T$ & $c 2$ \\
\hline Wa3 & 30.00 & 30.00 & 28.57 & 27.94 & 26.51 & 17.94 & 14.44 & 15.46 & 2.35 & 5.59 & 13.83 \\
\hline Wa7 & 30.00 & 30.00 & 23.81 & 14.44 & 12.06 & 10.79 & 11.75 & 19.02 & 2.36 & 3.28 & 11.42 \\
\hline Wa8 & 30.00 & 30.00 & 25.71 & 14.44 & 10.95 & 10.48 & 10.00 & 19.90 & 2.56 & 3.48 & 10.32 \\
\hline Wa9 & 30.00 & 30.00 & 25.24 & 18.41 & 13.02 & 11.90 & 8.73 & 21.74 & 1.30 & 3.77 & 9.45 \\
\hline Wa13 & 30.00 & 30.00 & 30.00 & 19.37 & 15.08 & 11.90 & 11.11 & 18.38 & 2.50 & 3.90 & 12.14 \\
\hline Wa14 & 30.00 & 30.00 & 30.00 & 17.62 & 17.30 & 11.75 & 11.11 & 17.00 & 3.89 & 3.75 & 13.27 \\
\hline Wa15 & 30.00 & 30.00 & 30.00 & 15.56 & 10.79 & 10.32 & 10.48 & 19.49 & 7.07 & 3.85 & 10.53 \\
\hline Wa16 & 30.00 & 30.00 & 30.00 & 30.00 & 12.54 & 11.11 & 11.90 & 18.85 & 10.01 & 4.77 & 11.15 \\
\hline Wa17 & 30.00 & 30.00 & 28.41 & 9.68 & 6.35 & 8.10 & 8.89 & 22.22 & 4.96 & 3.52 & 7.78 \\
\hline Wa21 & 30.00 & 30.00 & 30.00 & 16.67 & 11.43 & 8.73 & 8.57 & 20.80 & 3.80 & 3.84 & 9.45 \\
\hline Wa23 & 30.00 & 30.00 & 30.00 & 19.52 & 17.30 & 16.19 & 12.22 & 15.02 & 4.03 & 3.79 & 15.16 \\
\hline $\mathrm{Wa} 24$ & 30.00 & 30.00 & 30.00 & 29.37 & 16.03 & 10.48 & 11.90 & 18.80 & 4.64 & 4.77 & 11.17 \\
\hline Wa25 & 18.73 & 19.68 & 15.56 & 13.65 & 11.11 & 8.10 & 9.52 & 11.07 & 1.21 & 3.75 & 8.61 \\
\hline Wa27 & 30.00 & 30.00 & 30.00 & 26.83 & 16.83 & 10.95 & 11.90 & 18.86 & 2.56 & 4.64 & 11.20 \\
\hline Wa28 & 30.00 & 30.00 & 16.98 & 13.17 & 11.59 & 6.67 & 10.32 & 20.71 & 2.88 & 2.80 & 10.17 \\
\hline Wa29 & 7.46 & 8.41 & 11.43 & 12.86 & 16.51 & 12.70 & 10.79 & -5.82 & 2.56 & 2.68 & 13.26 \\
\hline Wa38 & 30.00 & 19.68 & 9.84 & 8.89 & 7.94 & 6.19 & 5.71 & 29.96 & 1.57 & 1.80 & 6.73 \\
\hline Wa39 & 30.00 & 23.02 & 19.21 & 12.70 & 11.90 & 10.48 & 10.48 & 28.62 & 0.89 & 1.93 & 9.89 \\
\hline Wa41 & 30.00 & 30.00 & 28.73 & 23.02 & 13.33 & 11.75 & 10.00 & 19.45 & 2.24 & 4.24 & 10.57 \\
\hline Wa42 & 30.00 & 27.14 & 22.38 & 19.52 & 12.22 & 9.68 & 10.16 & 22.77 & 0.98 & 3.63 & 8.47 \\
\hline Wa44 & 30.00 & 30.00 & 30.00 & 25.24 & 19.05 & 9.52 & 12.06 & 19.77 & 1.96 & 4.75 & 10.28 \\
\hline Wa46 & 30.00 & 29.05 & 27.62 & 25.87 & 17.62 & 14.29 & 11.75 & 17.69 & 1.66 & 4.67 & 11.78 \\
\hline Wa47 & 30.00 & 28.89 & 26.03 & 21.11 & 15.24 & 19.84 & 11.59 & 19.64 & 0.83 & 3.73 & 12.67 \\
\hline $\mathrm{Wa} 48$ & 30.00 & 30.00 & 30.00 & 30.00 & 14.44 & 16.51 & 16.35 & 13.50 & 7.20 & 4.53 & 16.55 \\
\hline Wa50 & 30.00 & 19.37 & 12.70 & 11.90 & 10.16 & 9.37 & 9.37 & 72.48 & 0.94 & 0.03 & 9.39 \\
\hline Wa54 & 30.00 & 14.92 & 16.98 & 11.11 & 13.17 & 8.10 & 9.68 & 26.11 & 1.74 & 1.73 & 9.52 \\
\hline Wa55 & 30.00 & 30.00 & 24.60 & 14.44 & 9.52 & 8.73 & 10.63 & 20.89 & 2.26 & 3.45 & 9.46 \\
\hline Wa56 & 30.00 & 30.00 & 30.00 & 29.52 & 21.90 & 25.08 & 13.81 & 18.82 & 1.75 & 5.96 & 9.46 \\
\hline Wa58 & 30.00 & 30.00 & 30.00 & 26.98 & 13.97 & 14.13 & 10.79 & 17.63 & 3.82 & 4.41 & 12.40 \\
\hline Wa59 & 27.46 & 27.30 & 19.05 & 18.25 & 10.48 & 8.25 & 10.16 & 20.78 & 1.11 & 3.46 & 8.43 \\
\hline Wa60 & 30.00 & 30.00 & 17.78 & 17.62 & 12.38 & 9.68 & 9.37 & 25.78 & 0.95 & 2.90 & 8.95 \\
\hline Wa61 & 26.67 & 30.00 & 30.00 & 18.73 & 12.38 & 10.63 & 10.79 & 19.07 & 3.73 & 3.90 & 11.14 \\
\hline Wa63 & 11.90 & 15.71 & 20.00 & 7.78 & 12.06 & 7.78 & 7.46 & NA & NA & NA & NA \\
\hline Wa64 & 30.00 & 26.19 & 13.81 & 12.22 & 10.32 & 9.84 & 9.84 & 20.00 & 2.77 & 2.46 & 10.42 \\
\hline Wa66 & 30.00 & 30.00 & 19.84 & 15.24 & 13.17 & 10.16 & 10.79 & 20.62 & 1.63 & 2.98 & 11.13 \\
\hline Wa67 & 27.30 & 12.22 & 14.92 & 9.68 & 5.40 & 4.92 & 4.44 & 22.56 & 1.25 & 2.52 & 4.55 \\
\hline Wa68 & 30.00 & 23.33 & 18.10 & 14.60 & 9.84 & 8.89 & 10.16 & 31.18 & 0.81 & 1.95 & 8.56 \\
\hline Wa69 & 27.30 & 19.05 & 18.57 & 16.35 & 10.95 & 9.05 & 10.00 & 18.95 & 1.42 & 2.95 & 9.52 \\
\hline Wa72 & 30.00 & 30.00 & 20.63 & 16.35 & 11.27 & 8.57 & 9.52 & 23.31 & 1.27 & 3.25 & 8.83 \\
\hline Wa76 & 30.00 & 30.00 & 30.00 & 23.49 & 22.22 & 20.63 & 11.75 & 18.50 & 2.23 & 5.15 & 11.41 \\
\hline
\end{tabular}




\begin{tabular}{llllllllllll} 
Wa77 & 30.00 & 30.00 & 28.89 & 29.21 & 19.05 & 18.10 & 15.87 & 12.69 & 4.80 & 4.66 & 16.96 \\
Wa78 & 30.00 & 30.00 & 18.10 & 14.13 & 14.60 & 10.79 & 10.79 & 17.84 & 3.91 & 2.81 & 12.51 \\
Wa79 & 30.00 & 30.00 & 17.78 & 12.06 & 11.11 & 10.00 & 10.95 & 19.31 & 4.06 & 2.85 & 10.97 \\
Wa81 & 19.84 & 12.70 & 10.32 & 8.41 & 8.89 & 8.41 & 7.14 & 15.55 & 1.70 & 1.70 & 8.01 \\
Wa84 & 30.00 & 30.00 & 30.00 & 27.14 & 14.29 & 15.56 & 13.02 & 16.95 & 2.20 & 4.75 & 13.00 \\
Wa86 & 30.00 & 26.98 & 20.00 & 17.94 & 14.29 & 13.02 & 11.75 & 25.53 & 0.76 & 2.43 & 11.21 \\
Wa88 & 30.00 & 30.00 & 28.73 & 13.33 & 11.43 & 10.48 & 10.48 & 19.23 & 4.49 & 3.58 & 10.78 \\
Wa89 & 30.00 & 30.00 & 23.49 & 12.86 & 11.27 & 8.41 & 10.63 & 20.45 & 2.46 & 3.28 & 9.97 \\
Wa93 & 30.00 & 30.00 & 30.00 & 15.08 & 19.05 & 12.38 & 13.65 & 14.97 & 16.82 & 3.67 & 15.03 \\
Wa95 & 28.25 & 23.33 & 14.29 & 10.32 & 10.79 & 10.16 & 10.16 & 18.90 & 2.17 & 2.38 & 10.23 \\
Wa99 & 30.00 & 27.46 & 30.00 & 26.51 & 13.49 & 10.32 & 10.95 & 18.55 & 3.55 & 4.52 & 10.61 \\
Wa100 & 30.00 & 30.00 & 24.44 & 23.02 & 12.06 & 12.38 & 13.65 & 17.28 & 1.89 & 4.00 & 12.15 \\
Wa101 & 30.00 & 27.14 & 27.30 & 17.62 & 20.63 & 12.86 & 13.97 & 19.20 & 0.85 & 3.78 & 12.31 \\
Wa102 & 30.00 & 30.00 & 30.00 & 26.51 & 12.86 & 8.41 & 11.11 & 20.28 & 3.35 & 4.47 & 9.76 \\
Wa103 & 30.00 & 30.00 & 18.41 & 16.03 & 10.95 & 8.57 & 8.89 & 24.43 & 1.20 & 3.02 & 8.63 \\
Wa104 & 30.00 & 30.00 & 23.17 & 25.56 & 17.62 & 14.76 & 15.08 & 18.10 & 0.90 & 4.12 & 12.97 \\
Wa105 & 30.00 & 30.00 & 27.62 & 16.03 & 11.90 & 9.84 & 12.38 & 18.84 & 2.95 & 3.64 & 11.25 \\
Wa109 & 30.00 & 30.00 & 30.00 & 23.65 & 16.83 & 13.02 & 10.95 & 19.42 & 1.65 & 4.45 & 11.12 \\
Wa111 & 30.00 & 30.00 & 26.35 & 30.00 & 12.22 & 12.70 & 11.90 & 19.48 & 2.30 & 4.60 & 10.55 \\
Wa114 & 16.98 & 23.97 & 16.51 & 16.67 & 12.70 & 10.32 & 10.79 & 9.54 & 1.56 & 4.29 & 10.26 \\
Wa117 & 30.00 & 30.00 & 23.65 & 21.90 & 10.32 & 10.16 & 11.27 & 20.05 & 1.64 & 3.94 & 9.76 \\
\hline
\end{tabular}




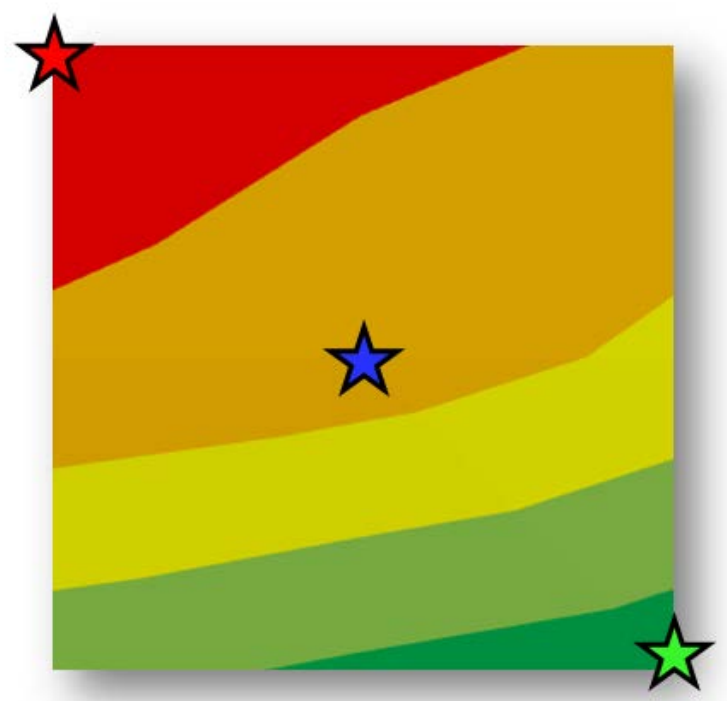

Dietary response surface for reproduction and evolutionary food types 


\section{CHAPTER V}

\section{EXPERIMENTAL EVOLUTION OF NUTRIENT-DEPENDENT REPRODUCTION IN DROSOPHILA MELANOGASTER}

Jelle Zandveld

Joost van den Heuvel

Bas Zwaan 


\section{ABSTRACT}

Dietary restriction (DR), often referred to as a reduction of food intake without malnutrition, extends lifespan in many organisms. However, how DR increases lifespan is poorly understood. As DR also affects other traits, for example reducing reproduction and increasing stress resistance, this suggests DR is an adaptive response. But experiments that test the evolutionary relevance of DR are not often performed and show contrasting results. New insights in ageing research showed that for a variety of species not only diet quantity, but also diet composition is mediating the lifespan response to diet. This new insight of nutritional complexity could explain the differences between studies and could be used to further assess the adaptive significance of DR and its dynamics in life-history evolution..

Taking these new insights into account, here, we used an experimental evolution approach to assess the effects of three adult diets on nutrient-dependent lifespan and reproduction patterns in Drosophila melanogaster. For all replicates and all evolutionary diets, we observed that reproduction increased on the population's evolutionary diet, clearly indicating adaptation to these conditions. However, these evolutionary changes in reproduction were not accompanied with a consistent changed lifespan response to evolutionary diet. Instead, the evolutionary diet treatment showed strong lineage-specific effects not only in the lifespan response, but also in other life-history traits, such as fat content, development time, and starvation resistance. Our results are in concordance with that evolutionary adaptations to improve diet-dependent reproduction in Drosophila, involves different life-history adaptations, but that not specifically includes a changed lifespan response to the evolutionary diet. 


\section{INTRODUCTON}

\section{Evolutionary environments that alter life-history traits}

Life-history traits are affected by both biotic and abiotic factors, the phenotypic trait value varies dependent on the environment (Maret \& Collins 1997; Marshall \& Sinclair 2009; Bento et al. 2010; May et al. 2015). Some of these plastic responses are a popular subject of study in laboratory experiments, for example, dietary restriction (DR), which is often defined as a reduction of food intake without malnutrition. Typically, DR increases lifespan or improves health in many organisms ranging from yeast to primates (McCay et al. 1935; Klass 1977; Weindruch et al. 1986; Weindruch \& Walford 1988; Chippindale et al. 1993; Colman et al. 2009; Mattison et al. 2012) and perhaps in humans as well (Most et al. 2016). Also other traits are affected by DR, such as locomotion (Ingram et al. 1987; Bross et al. 2005), response to stresses, reproduction (Emran et al. 2014; Van den Heuvel et al. 2014; Solon-Biet et al. 2014), and body composition (Skorupa et al. 2008; Solon-Biet et al. 2014). Although these effects of DR on the phenotypic level are well investigated, how DR increases lifespan and health is not yet fully understood.

\section{Lifespan and reproduction trade-offs}

Laboratory selection experiments with Drosophila populations often established an evolutionary connection between reproduction patterns and lifespan; namely that selection for late reproduction extends lifespan (Luckinbill et al. 1984; Rose 1984; Partridge et al. 1999; Stearns et al. 2000; May 2016), direct selection for increased lifespan decreased early fecundity (Zwaan et al. 1995), and also selection for increased starvation- and desiccation resistance resulted in a correlated increase in longevity and a decreased reproduction (Rose et al. 1992). This shows a genetic correlation between lifespan and reproduction, that often trade off.

Also upon diet change lifespan and reproduction often show a negative relationship, but which is more likely a physiological trade-off, acting through a different allocation of resources to lifespan or reproduction rather than a genetic interrelationship. The effects of DR are not only restricted to lifespan and reproduction, it also increases stress resistance and locomotion (Bross et al. 2005; Emran et al. 2014). This may represent an adaptive life-history strategy in times of food shortage. Harrison \& Archer (1988) and Holliday (1989) hypothesized that for organisms in a nutritional variable environment in times of food shortage it is adaptive to invest the limited nutrients in somatic maintenance rather than in reproduction (Harrison \& Archer 1988; Holliday 1989), an idea that is supported by population models for different species (Shanley \& Kirkwood 2000; Mangel 2001; Van den Heuvel 2014). However, not much research is performed to connect evolutionary genetics with life-history plasticity to nutrient availability.

\section{Evolution of lifespan and reproduction responses to diet}

Natural genetic variation for both the lifespan and reproduction response to diet is observed in different species (Stastna et al. 2015; Sutphin \& Kaeberlein 2008; Van Diepeningen et al. 2008; Chapter 4 this thesis; Metaxakis \& Partridge 2013). Different natural populations of a species can 
be subjected to different selection pressures, leading to adaptation to local conditions and an increased intraspecific variation. For example, in the fungus Podospora anserina, we observed ample variation between strains in their lifespan response to diet, and interestingly, lifespan on control food correlated with the lifespan response to diet (Chapter 4, this thesis). Similarly in Drosophila, a laboratory population $(w D a h)$ showed higher reproduction and also longer lifespan when compared to wild-derived populations (Metaxakis \& Partridge 2013). This wDah population also seemed to respond differently to diet change when compared to the shorter-lived natural strains: their optimal lifespan was achieved on a relatively higher level of nutrition. Together, these data suggest that populations evolved in conditions more similar to the nutritional conditions tested, may live longer, reproduce more, but may also have a changed response to nutrients. Evolutionary adaptation to a certain nutritional condition could improve lifespan and reproduction locally, but deviating from this condition may result in a changed lifespan and reproduction response.

\section{Effects of nutrient-dependent evolution}

In line with this, females of populations subjected to long-term laboratory culturing on two different food concentrations indeed reproduced more and also lived longer on their evolutionary conditions (Chapman et al. 1994). In a more recent study, however, laboratory culturing for three different (adult) food levels was applied (Zajitschek et al. 2016) and although an interaction between evolutionary diet and the male's lifespan response to diet was observed, this effect was lineage-specific and did not point at a longer lifespan on the evolutionary diet (Figure S1). Interestingly, an increased reproductive performance was observed for males evolved on a DR-like food type that was irrespective of food type (Zajitschek et al. 2016). One possible explanation for these contrasting results could be a differential life-history strategy between the experiments. Organisms may evolve convergent strategies that are both adaptive for a similar evolutionary condition (Stearns 1992; Cooper et al. 2008; Klepsatel et al. 2014). For example, other previous evolution experiments in Drosophila also resulted in differential lifespan responses between replicate lineages of the same evolutionary condition (e.g. Partridge et al. 1995; Baldal et al. 2006; Doroszuk et al. 2010). Furthermore, for multiple life-history traits including lifespan and reproduction, there are strong interactive effects between laboratory assay conditions and strains (Ackerman et al. 2001). This shows that the exact traits on which selection acts can be very different between laboratory cultures and might explain the differences observed, an imply that an organism's life-history strategy as a whole matters, and not the individual traits.

\section{Nutritional geometry}

Recent insights in the relationship between nutrition and ageing also provide a framework that may help improve the comparability between studies. The Geometric Framework (GF; Simpson \& Raubenheimer 2012) is a useful tool to present how both the abundance and combination of different nutrients simultaneously affect a phenotype. In the GF, each nutrient under 
consideration is assigned an axis in multidimensional space and the abundance of that nutrient increases with distance from the origin. This concept is successfully applied in a variety of species including Drosophila, and illustrated that not only abundance but also nutrient combinations alters lifespan (Lee et al. 2008; Skorupa et al. 2008, Solon-Biet et al. 2014; Piper et al. 2017). Moreover, by incorporating this same concept in our study design, we have demonstrated how it may be possible to resolve apparently contradictory interpretations of how one genotype can affect diet responses in Drosophila (Zandveld et al. 2017, Chapter 2, this thesis). So to adequately test whether a genotype modifies the lifespan response to diet, a broad range of diets that capture a large proportion of nutrient space should be explored. Including this new insight to analyses of genetic change by experimental evolution of any kind, could help resolving contradictory outcomes between studies, but in particular those with different nutritional conditions observed (Chapman et al. 1994; Ackerman et al. 2001; Zajitschek et al. 2016).

\section{This study}

In this study we maintained replicate populations on three distinct evolutionary diets that had similar caloric content but differed greatly in sugar to yeast ratio. To test whether experimental evolution to a changed nutrient-dependent reproduction pattern (i.e. local adaptation) also affects lifespan on the evolutionary conditions, we measured the lifespan response to ten food types that differ in both nutrient quantity and composition, thereby taking the new insights of nutritional geometry into account. Apart from these measurements, we also determined effects of evolutionary nutrition on multiple life-history traits, namely development time, body composition and starvation resistance. Using this set up we can identify whether the genetic interrelationship between reproduction, lifespan or possibly other life-history traits, relate to the lifespan and reproduction effects observed upon diet change.

\section{MATERIAL \& METHODS}

\section{Fly husbandry}

The ancestral population was derived from a laboratory population that in turn originated from crosses between six wild populations collected across Europe, as previously described by (May et al. 2015). Eggs of this ancestral population were set up, and the resulting adult flies were used for phenotyping the first generation (Figure 1), and for founding the twelve experimental evolution populations (approximate population size $\mathrm{N}=400$ ). For every generation, all fly populations develop as larvae on standard food for 10 days, and all emerged adults were then transferred to population bottles containing their evolutionary food type. To reduce mortality due to crowding but to still maintain a large population size, the adult evolutionary medium was located on the side of each population bottle to increase the food surface. Adults were kept for three days in population bottles and on the fourth day were transferred to cages with Petri-dishes containing their evolutionary food type. For each population $42 \mu \mathrm{l}$ of all eggs laid over a 24 hour period were 
transferred on day 5 to a new bottle containing standard food, by using the method of Clancy \& Kennington (Clancy \& Kennington 2001). These eggs developed again to adults to give rise to the next generation. For a more detailed protocol see Supplemental Data S1. All experiments were performed under standard laboratory conditions $\left(25^{\circ} \mathrm{C}, 65 \%\right.$ humidity, $12 \mathrm{~h}: 12 \mathrm{~h}$ light:dark cycle).

\section{Food types}

For all food types standard ingredients were added per litre, $15 \mathrm{~g}$ agar, $3 \mathrm{ml}$ propionic acid, $30 \mathrm{ml}$ Nipagin in 95\% ethanol, but the quantities of yeast (Fermipan Instant Yeast Red Label) and sucrose differed between diet treatments.

Evolutionary diets - The experimental evolution populations were subjected to three adult food types with a similar caloric content but different sugar to yeast (S:Y) ratio's; namely, a low S:Y ratio (50 gr. $\mathrm{l}^{-1}$ sucrose, 200 gr..$^{-1}$ yeast, referred to as 'evoL'), an intermediate ratio (standard food, 100 gr. $\mathrm{l}^{-1}$ sucrose and 100 gr. $\mathrm{l}^{-1}$ yeast, referred to as 'evoI') and a high S:Y ratio (200 gr. $\mathrm{l}^{-1}$ sucrose, 50 gr..$^{-1}$ yeast, referred to as 'evoH'). For every generation, larvae developed on standard food.

Assay diets - Our standard food consists of, and the nine food varied in the combination of sucrose and yeast concentrations following Skorupa et al. (2008), and varied between 50 gr. $\mathrm{l}^{-1}, 100$ gr. $\mathrm{l}^{-1}$ and 200 gr. $\mathrm{l}^{-1}$ for both yeast and sugar For the $24^{\text {th }}$ generation, a tenth food type was used, and contained 50 gr..$^{-1}$ sugar and 150 gr..$^{-1}$ yeast.

\section{Phenotypes: lifespan, reproduction, development time, body composition, and starvation resistance}

Prior to all experiments, the populations were maintained for two generations at the same density and on standard food to prevent any possible influence of the maternal/paternal diet.

For all lifespan analyses (measured in the $1^{\text {st }}, 24^{\text {th }}$ and $46^{\text {th }}$ generation), flies were set up on standard food to mate for 48 hours. On day 3, after mild $\mathrm{CO}_{2}$ anaesthesia, females were allocated to one of the assay diets. In the first generation, lifespan was assessed on nine diets, in the $24^{\text {th }}$ generation on ten diets, i.e. the same nine diets plus an additional diet (see above), and in the $46^{\text {th }}$ generation all populations were tested on the three evolutionary diets. To score lifespan, three times per week the flies were placed onto fresh media and for each transfer deaths were recorded.

For the first and 24th generation, after 6 days on their diet, reproduction was determined in the lifespan cohort by counting the number of eggs laid in 24 hrs per vial. For generation 42 and 46, reproduction was determined by counting the number of eggs laid in 24 hrs per vial on day 5 on their diet treatment, using continuously mated females. In generation 42 , flies were transferred to new food on the third and fourth day after set up (note that this is similar to the evolutionary protocol), but in generation 46 on the second and fourth.

Development time was measured after 42 generations, for 10 replicate vials containing $6 \mathrm{ml}$ standard food. Per vial 100 eggs were set up and the average development time per vial was recorded and used for analysis. 
After 26 generations, body composition was measured for each population for six replicate vials with each containing 10 once-mated female flies. After 6 days flies were snap frozen, and measurements were taken for the average of ten flies per vial. First, wet weight was measured, then after 24 hours of air-drying in a glass vial at $40^{\circ} \mathrm{C}$, dry weight and water content were calculated. Body fat was removed by applying $1 \mathrm{ml}$ dichloromethane:methanol (2:1 ratio) to each vial. After incubation for 72 hours at $40^{\circ} \mathrm{C}$, the vials were dried for another 24 hours. Then, fat-free weight and fat content could be estimated.

After 36 generations, starvation resistance was determined using 100 females of each population on agar-water containing 30ml/L Nipagin and $3 \mathrm{ml} / \mathrm{L}$ propionic acid. Flies were transferred three times a week and deaths were recorded daily.

An overview of all assays is provided in Table S1.

\section{Statistical analyses}

All statistical analyses were performed using R. To test lifespan effects of, and interactions, between evolutionary treatment, population, sucrose and yeast concentration, a mixed-effect cox proportional hazard test was performed (coxme package) with population as a random factor. To test gender and population specific-effects on lifespan of changing the sucrose and yeast nutritional environment, a proportional hazard test was performed (coxph package). For reproduction, per generation the total egg count data for each vial was summed and log transformed. An analysis of variance (Anova) was performed to test for effects of, and interactions between, evolutionary treatment, population, sucrose and yeast concentration. A linear mixed-effects model (lme package) was applied to test for interactions between evolutionary treatment and nutrientdependent effects of reproduction for all generations together. For development time, the average development time per vial was estimated and an analysis of variance (Anova) was performed to test for effects of evolutionary treatment and population. Also for each body composition phenotype an analysis of variance (Anova) was performed to test for effects of evolutionary treatment and population. To test the effects of evolutionary treatment and population on starvation resistance, a cox proportional hazard test (survival package) was performed. To explain effects of evolutionary nutrition a principal component analysis (PCA) was performed on the scaled values of all life-history traits measured, with the exception of reproduction.

\section{RESULTS}

Reproduction and lifespan responses of the ancestral population

In a parallel cohort of the ancestral population when setting up the experimental evolution populations, we tested the effects of a range of nine diets on male and female lifespan (see materials and methods; similar conditions as in Zandveld et al. 2017). We observed a three-way interaction between gender, dietary sugar, and yeast $\left(X^{2}=9.88\right.$, d.f. $\left.=4, p=0.042\right)$. Female lifespan peaked at 
lower sugar concentrations and intermediate yeast levels, while males had a broader lifespan peak, from low to intermediate sugar and yeast levels, and had in general a reduced response to both differential sugar and yeast levels (Figure 1). However, these effects of gender on the lifespan response to diet differed starkly for separate diet dimensions (Figure 1, Table S1).

For female reproduction, both sugar and yeast significantly affected reproduction (Anova, $\mathrm{p}<0.001$, Figure 1, Table S1). Reproduction peaked on relatively low sugar:yeast ratios, and decreased along the same isocaloric axis with increasing sugar:yeast ratios (i.e. treatment 3, Figure 1c).
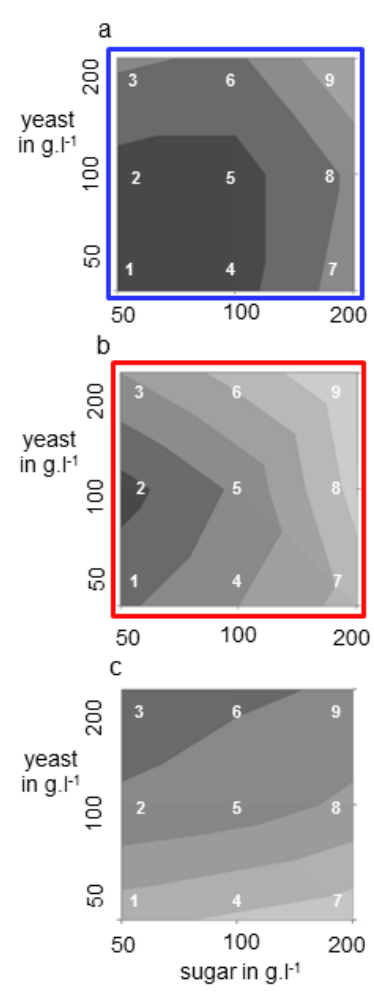

Lifespan (in days)
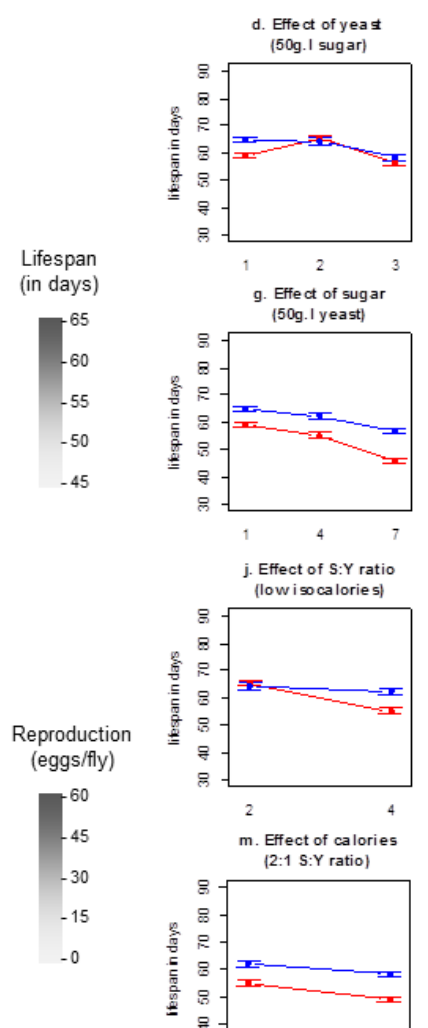
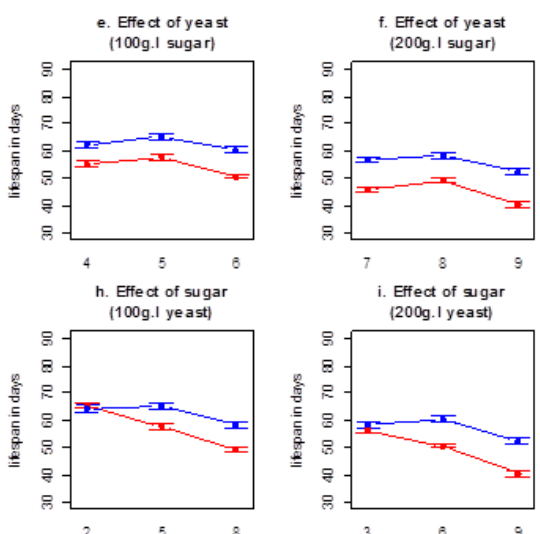

k. Effect of S:Y ratio (medium isocalories)
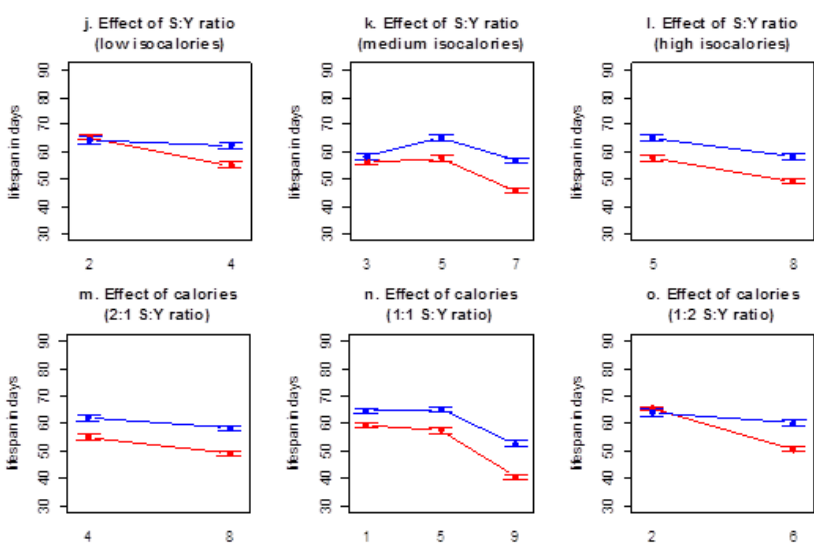

n. Effect of calorie
(1:1 S:Y ratio)

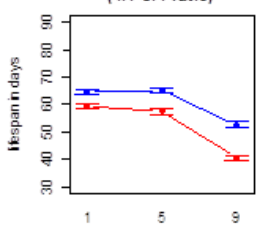

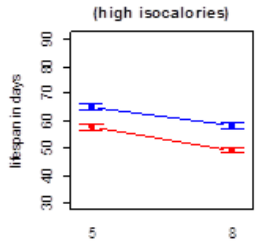

o. Effect of calories (1:2 S:Y ratio)

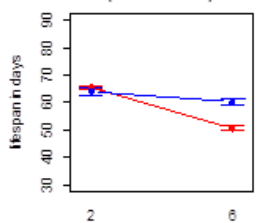

Figure 1: Response surface of the ancestral population (i.e. prior to experimental evolution); lifespan of males (a) and females (b) and female reproduction (c) on nine food types capturing both lifespan changes through differential concentrations of dietary sugar and yeast. (d-o) Lifespan interaction plots (mean $+/$ - standard error) display how the lifespan response to different nutrient orientations varies between the two sexes (blue lines represent male, red lines female flies). Numbers on $\mathrm{x}$-axis correspond to nutrient orientations of figures a-c. Thus, d,e, f displays effect of dietary yeast on different sugar levels; $\mathrm{g}, \mathrm{h}, \mathrm{i}$, effect of dietary sugar on different yeast levels; $\mathrm{j}, \mathrm{k}, \mathrm{l}$ effect of $\mathrm{S}: \mathrm{Y}$ ratio on different caloric levels; and m,n,o effect of calories on different S:Y ratio's. 


\section{Reproduction response to nutrients after experimental evolution}

The food conditions along this diet dimension showed the largest decrease in reproduction. The largest effects of a different evolutionary nutrition were most likely to occur for this diet orientation. Therefore, we tested the effect of evolutionary nutrition on three diets along this axis. Specifically, to investigate whether evolution on specific adult isocaloric diets resulted in local adaptation, nutrient-dependent reproduction for the evolved populations on the three evolutionary diets was measured. We observed a significant evolution by diet interactions $($ lmer, $p<0.01$, Figure 2, Table S1), i.e. reproduction for populations evolved on a low S:Y ratio (evoL, Figure 2 red line) had an increased reproduction on their food condition compared to the other populations, but also populations evolved on relatively high S:Y ratios (evoH, Figure 2 green line) laid more eggs on their evolutionary food type when compared to populations from the other evolutionary conditions.

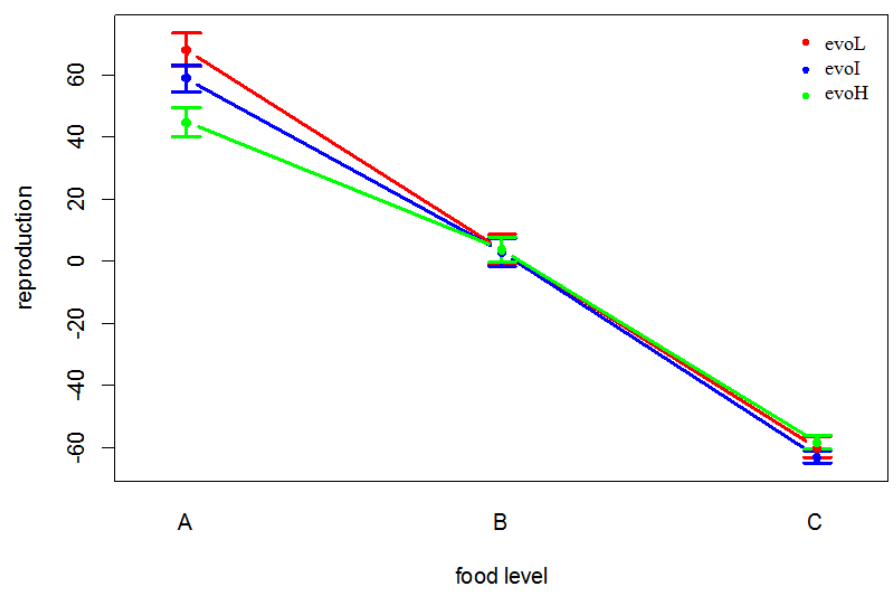

Figure 2: Effects of evolutionary background on nutrient-dependent reproduction responses for the three evolutionary food levels summarized for the evolutionary food types over the three generations tested $\left(24^{\text {th }}, 42^{\text {nd }}\right.$ and $46^{\text {th }}$ generation). Residuals of generation effects on nutrient-dependent reproduction are shown. In figure S2 the effects of evolution treatment on nutrient-dependent reproduction are shown per replicate generation.

\section{Nutrient-dependent lifespan responses are not consistently affected by evolutionary diets}

These evolutionary changes for reproduction were not accompanied by consistent changed responses in lifespan to diet after 24 (coxph, $p>0.1$, Figure 3), nor after 46 generations (coxph, $p>0.1$, Figure S3). However, within the evolutionary treatments large effects in the response to diet were observed for different replicate lineages ( $c 0 x p h, p<0.001$, Figure 3). Most replicate lineages showed an increase of lifespan upon a sugar decrease and at intermediate yeast levels, although exceptions within every were observed as lifespan could peak at low sugar and low yeast levels. As a consequence, when testing effects of lineage-specific effects for separate diet dimensions (such as 
applied in Figure 1d-o), we observed very distinct responses to diet between the different replicate lineages (e.g. Figure 3).
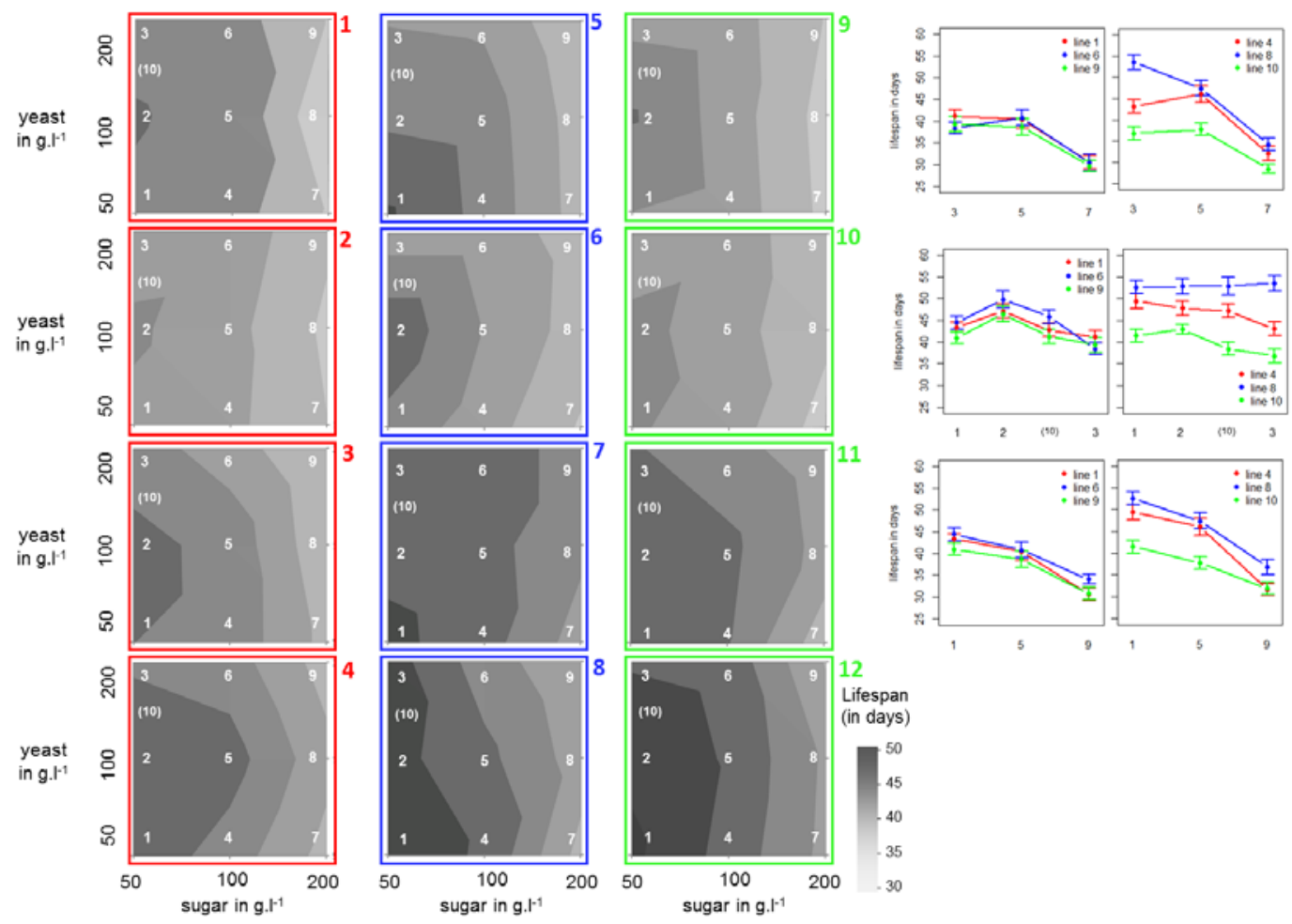

Figure 3. Left: lifespan response surfaces per population after 24 generations of experimental evolution. For each population the mean lifespan for nine diets that differ in both the combination and concentration of dietary sugar and yeast are shown, darker colours represent a longer lifespan. The coloured boxes that surround each response surface indicate the evolutionary conditions, i.e. red: low S:Y ratio; blue intermediate S:Y ratio, and green high S:Y ratio. Right: interaction plots for three diet orientations for populations with a similar evolutionary diet show large population-specific effects for the lifespan response to diet. The numbers correspond to the ten treatments of response surface. The upper panel represents the response to the three evolutionary conditions, similar caloric content but different S:Y ratio; the central panel shows an often applied DR protocol, changing the S:Y ratio by diluting the yeast component; the lower panel demonstrations the effects of increasing calories for a similar S:Y ratio. 


\section{Effects of experimental evolution on other life-history traits}

Similarly, for other measured life-history traits (development time, starvation resistance, body weight and body composition), we also observed lineage-specific effects, but larger consistencies within evolutionary diet treatments. Although the development time for populations evolved on high sugar:yeast ratio $(\mathrm{evoH})$ showed two clearly distinct responses between replicate lineage (Anova, $p<0.01$, Figure 4), the effects of the other evolutionary diets were more consistent between populations. Namely, flies evolved on low S:Y ratios (evoL) extended development time compared to flies evolved on intermediate S:Y ratios (evoI) (Anova, $p<0.05$ ). Similarly, for the response to starvation resistance (SR), the same lineages within the evoH conditions that exhibited extended development time, lived longer under SR ( $c o x p h, p<0.01$, Figure 4) and normal conditions (Figure 3). However, no consistent effects of evolutionary treatment on SR were observed (coxph, $\mathrm{p}<0.1)$. Moreover, fly body weight and body composition differed between populations within evolutionary treatments, also here consistent changes with evolutionary nutrition were observed (Table S3). For example, dry weight of flies evolved on high S:Y (evoH) conditions showed a general lower dry weight compared to the other evolution treatments (Figure 5a, Table S3) and consistent for all replicate lineages within evolutionary diet. Furthermore, although within evoH lineage 11 and 12 (which had longer development time, longer lifespan, and higher SR) had a seemingly lower fat percentage (Figure 5), this was not significant (Anova, $p>0.1)$. However, evoH populations had in general a slightly higher fat percentage, but this was not significant (Anova, $p<0.1)$.

a. Development time

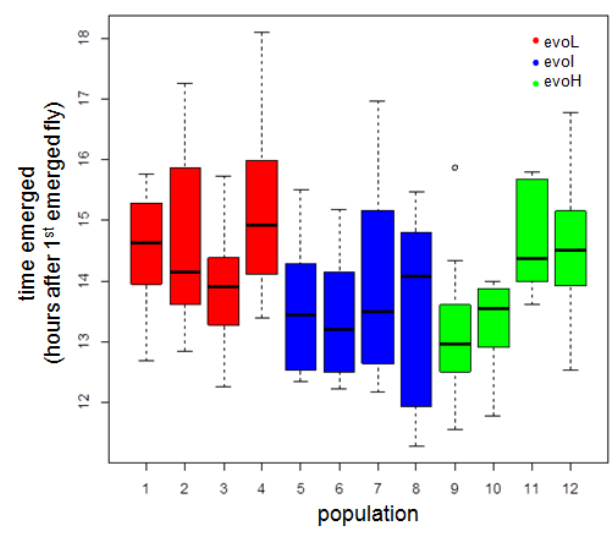

b. Starvation resistance

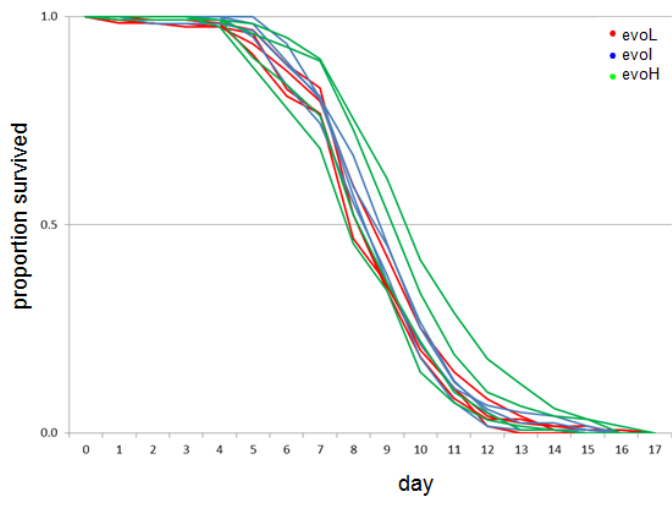

Figure 4 (a) Boxplots of the effects of evolutionary diet on development time per population, measured in hours from the first emerged adult fly. (b) The effects of evolutionary diet on starvation resistance per population, represented as survival proportion per day. 
a. Wet weight

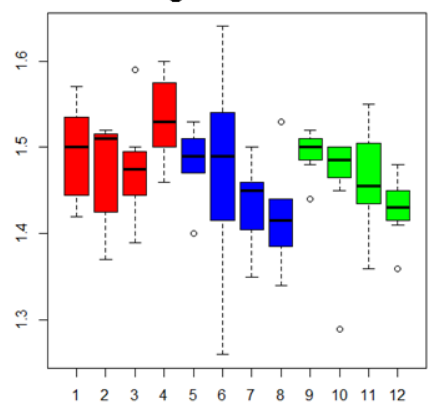

d. Fat percentage

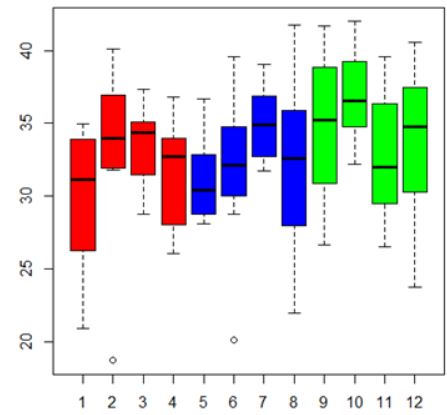

b. Dry weight

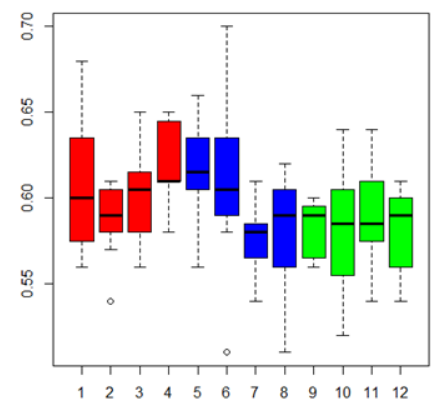

a. Fat-free weight

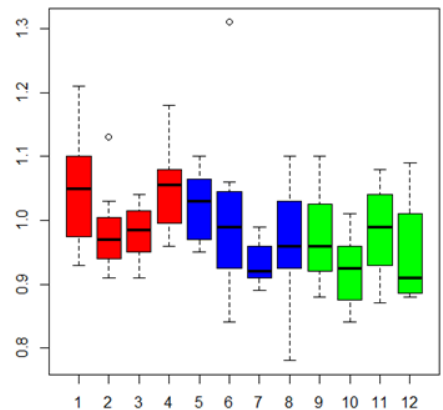

c. Water content

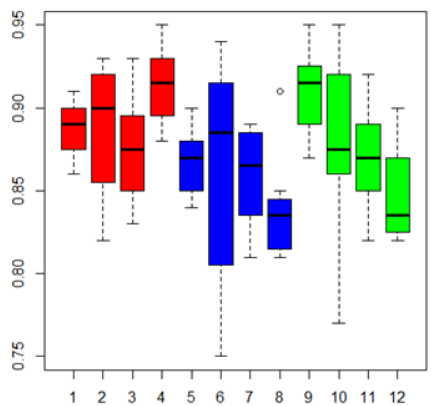

Figure 5: Boxplots of all measured body composition phenotypes for each of the twelve populations. Per population the averages per fly are plotted; (a) Wet weight in mg; (b) Dry weight in mg; (c) water content in $\mathrm{mg}$; (d) fat percentage in percent and (e) fat free weight in mg. Colours represent evolutionary diet conditions: red $=$ evoL, blue $=$ evoI, and green $=e v o H$.

\section{Principal component analysis}

To capture these responses in a more integrative life-history perspective (in which potentially a set of combinations of traits contributes to local adaptation), we performed a principal component analysis (PCA) on all data of life-history traits per replicate lineage, but excluded egg production (Figure 6). The first two PC's explained more than $75 \%$ of the variation and showed strong lineagedependent patterns. The evoL lineages showed a more consistent pattern than the other evolutionary treatments. Within both evoI and evoH two different patterns are observed, which indicate close resemblance of at least one other but not all replicate lineages within evolutionary treatment (Figure 6). 


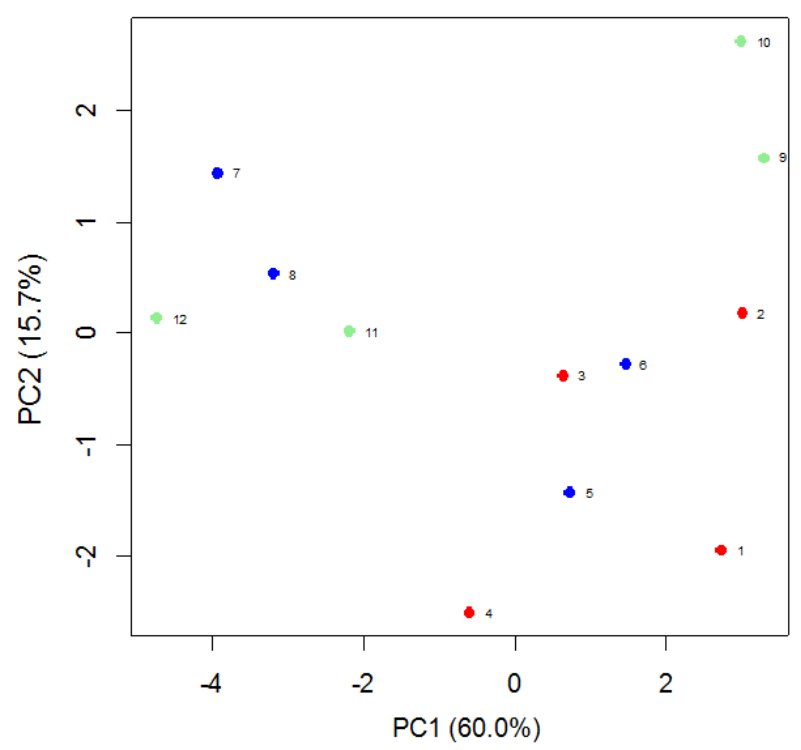

Figure 6: Principle component analysis (PCA) uncovers PC1 and PC2 that, respectively, describe $60.0 \%$ and $15.7 \%$ of the variation within evolutionary conditions for the replicate lineages differing in lifehistory traits. Evolutionary treatment is indicated in the different colors: red = evoL, blue = evoI, and green $=$ evoH. Separate lineages are indicate by numbers.

\section{DISCUSSION}

In this study, we showed that experimental evolution in different adult nutritional conditions in Drosophila melogaster populations resulted increased reproduction on diets that matched the evolutionary diet ("local adaptation"). Interestingly, by exploring a broad range of diets we showed that the evolutionary changes were not accompanied with a consistently changing lifespan response to diet, but rather, we observed lineage-specific changes in lifespan and other life-history traits. This suggests that adaptation to nutrients in Drosophila is driven mainly through selection on reproduction, but that effects on the general life-history strategy can be achieved via concerted changes in combinations of traits. Crucially, however, these life history changes are not necessarily accompanied by a changed lifespan response to diet.

\section{Reproduction and lifespan responses in the ancestral population}

In previous work, we showed that when testing whether a genetic intervention affects the lifespan response to diet, multiple diets should be assessed. Namely, it can highly depend on the nutrientdimension under consideration whether genotypes affect the phenotypic response (Zandveld et al. 2017). In this study, we applied this approach in both our ancestral and evolved populations. First, 
we determined lifespan and reproduction in the ancestral population on nine different food types and showed that on a low sugar:yeast ratio diet female reproduction is increased and, within the diet range tested, reproduction had the largest decrease along the same isocaloric axis with an increasing S:Y ratio (Figure 1). Therefore, when testing the effects of evolutionary adult nutrition, the largest evolutionary effects of nutrient conditions are most likely to occur for along this axis. We also measured lifespan in the ancestral population for both sexes on the same range of diets and showed that although males and females had their lifespan optimum at low sugar/intermediate yeast diets, males showed in general a lower response to a diet change. However, the magnitude of this difference changed starkly per diet orientation under consideration (Figure 1d-o). Similar to assessing the effects of mutant genotypes (Zandveld et al. 2017), this again stresses the importance of measuring lifespan on a broad range of food types, also for gender effects.

\section{Effects of evolutionary nutrition}

In this study we investigated the genetic effects through evolutionary adaptation (using standing natural genetic variation) to different diets. To our knowledge, this is the first study using adaptation to isometric diets and then subsequently assessing the nutritional reaction norm using a very broad range of diets. For reproduction, no clear effects of evolutionary treatment were observed on the intermediate diet, which can be explained by the smaller nutritional distance with the other evolutionary diets. But pre-adaptation seems a valid option, as the nutrient composition of the intermediate diet (i.e. $100 \mathrm{~g} . \mathrm{l}^{-1}$ sucrose, $100 \mathrm{~g} . \mathrm{l}^{-1}$ yeast) is nutritionally nearest to the standard diet used prior to evolution (i.e. $100 \mathrm{~g} \cdot \mathrm{l}^{-1}$ sucrose, $70 \mathrm{~g} . \mathrm{l}^{-1}$ yeast) (May et al. 2015). In contrast to the absence of evolutionary effects on intermediate diets, we showed strong signs of local adaptation for the two other diets and, not surprisingly, this effects was most pronounced for the two nutritionally most distinct populations (evoL: low S:Y ratio vs evoH: high S:Y ratio) (Figure 2). Although this effect of local adaptation was not observed in a comparable experiment performed with males (Zajitschek et al. 2016), similar results were acquired when females were used (Chapman et al. 1994). Indeed, gender differences in the lifespan and reproduction response to diet are well recognized (Magwere et al. 2004; Bros et al. 2005; Zajitschek et al. 2013; Regan et al. 2016;), and also clearly demonstrated in the ancestral population of this study (Figure 1), but are however, poorly understood (Burger \& Promislow 2004). But it seems that where males have apparently an optimal evolutionary diet, females show stronger pleiotropic effects for their reproductive performance for different environments.

For the lifespan response to diet, we showed no consistent effects of evolutionary diet, but much stronger lineage-specific responses (Figure 3). Our results are therefore in concordance with previous findings that although reporting effects of evolutionary nutrition on the mean lifespan response of males (Zajitschek et al. 2016), also strong effects on the lifespan response for replicate lineages were measured (Figure S1). On the other hand, Chapman et al. (1994) reported a clear increase of lifespan on the flies' evolutionary diet (Chapman et al. 1994). It might be that the lifespan responses to diet is affected after more prolonged experimental evolution (approx. 100 
generations by Chapman et al. (1994)) and is in line with the comparison of natural populations to a laboratory adapted strain showing different 'lifespan by diet' optima (Metaxakis \& Partridge, 2013). Nevertheless, neutral effects of evolution could also explain these effects, namely, when a trait is not adaptive or relevant for a certain environment it may evolve neutrally, i.e. standing genetic variation differentiates between lineages due to random genetic drift (Chalambor et al. 2007).

So in conditions where flies live relatively long, such as in our study and that of Zajitschek et al. (2016; i.e. median lifespan between 30 and 60 days), lifespan is more likely to be evolving neutrally. In the situation of the study of Chapman et al.(1994) however, where flies lived relatively short (i.e. median lifespan between 15 and 20 days), lifespan was probably a more relevant trait in these conditions. As we also observed no clear changes between the populations after 42 generations (Figure S3), this last scenario seems more likely and shows that the mechanisms through which lifespan responds to diet are not genetically linked with the nutrient-dependent patterns of reproduction and therefore not part of the adaptation to the evolutionary nutrient conditions. This is also in line with other evolutionary treatments that not directly select for a lifespan change but for other life-history traits; also these studies observed no effects on Drosophila lifespan in the laboratory (Ackerman et al. 2001; Baldal et al. 2006; Doroszuk et al. 2010). Similarly, also for evolutionary adaptations in the wild lifespan is not necessarily linked to the fly's life-history (Klepsatel et al. 2014).

\section{Opportunities to parse apart lifespan and reproduction effects}

The different lifespan responses between populations are relatively stable over multiple generations (Figure S3). As this variation in lifespan responses between lineages is accompanied with a more constant reproduction response between populations, this could open up the opportunity to study the mechanisms of this decoupling of lifespan and reproduction. Genetic variation between populations within evolutionary treatment can be associated to the effects of lifespan only, and importantly, without interference of the reproduction response. For example, when measuring gene transcription, significant changes associated with a differential lifespan, can be decoupled from the more stable reproduction response between populations. So for further mechanistic understanding of the lifespan response to diet, using the specific contrast between these populations can give valuable insights in the mechanisms of nutrient-dependent lifespan.

\section{Other life-history traits also show evolution-dependent changes}

Apart from the observed lineage-specific effects and lack of consistent effects on the lifespan response for lineages within evolutionary diets, also other life-history traits measured in this study, such as development time, body composition and starvation resistance, showed populationspecific effects. However, for these traits a more consistent change upon the evolutionary treatments was observed (Figure 5, Figure 6). These consistent evolutionary effects suggest a less neutral role of these traits in adapting to the different isometric diets. Namely, for development 
time, starvation resistance, and general lifespan, among replicate lineages within evoI and evoH, showed two clearly separated patterns were observed, demonstrated by the PCA in Figure 6. Because nutrient-dependent reproduction patterns were relatively consistent between populations (Figure 2, Figure S2), this suggests at least two different life-history strategies could be involved in the adaptation to a high S:Y ratio diet. Evolution on a low sugar:yeast ratio diet (evoL), showed that all populations evolved a delayed development time compared to evoI populations, and also showed more consistent effects for starvation, lifespan, and body composition for the replicate lineages within the evolutionary treatment. Our results are consistent that upon experimental evolution on different nutritional conditions, flies can adapt multiple life-history strategies to achieve a similar nutrient-dependent reproduction pattern on high S:Y ratios (Figure 6). The more consistent life-history phenotypes for populations evolved on a low S:Y ratio diet, may suggest that selection for improved reproduction on this food type only favours one life-history strategy.

\section{CONCLUSION}

In this study we showed that evolutionary adaptation to three nutritionally distinct diets consistently improves reproductive fitness in the selective, evolutionary environment, but that such adaptation does not necessarily include concerted changes in the lifespan response to diet. In addition, by using a broad range of diets, we showed that the lifespan responses were highly dietindependent and largely explained by replicate lineage, but not evolutionary-specific effects. The observed lineage-specific effects on lifespan but also on other important life-history parameters suggest that adaptations to improved nutrient-dependent reproduction can be achieved via different life-history routes but is unlikely to change lifespan responses to diet. 


\section{SUPPLEMENTAL MATERIAL}

\section{Figure $\mathbf{S 1}$}

Lifespan effects Zajitschek et al. 2016) further explored

\section{Figure S2}

Nutrient-dependent reproduction patterns for different generations

\section{Figure S3}

Nutrient-dependent lifespan patterns for different generations

\section{Data S1}

Methods experimental evolution maintenance

\section{Table S1}

Overview all assessed phenotypes per generation

\section{Table S2}

Statistical analyses

All data is available upon request 


\section{Figure S1}

\section{Lifespan effects Zajitschek et al. 2016) further explored}
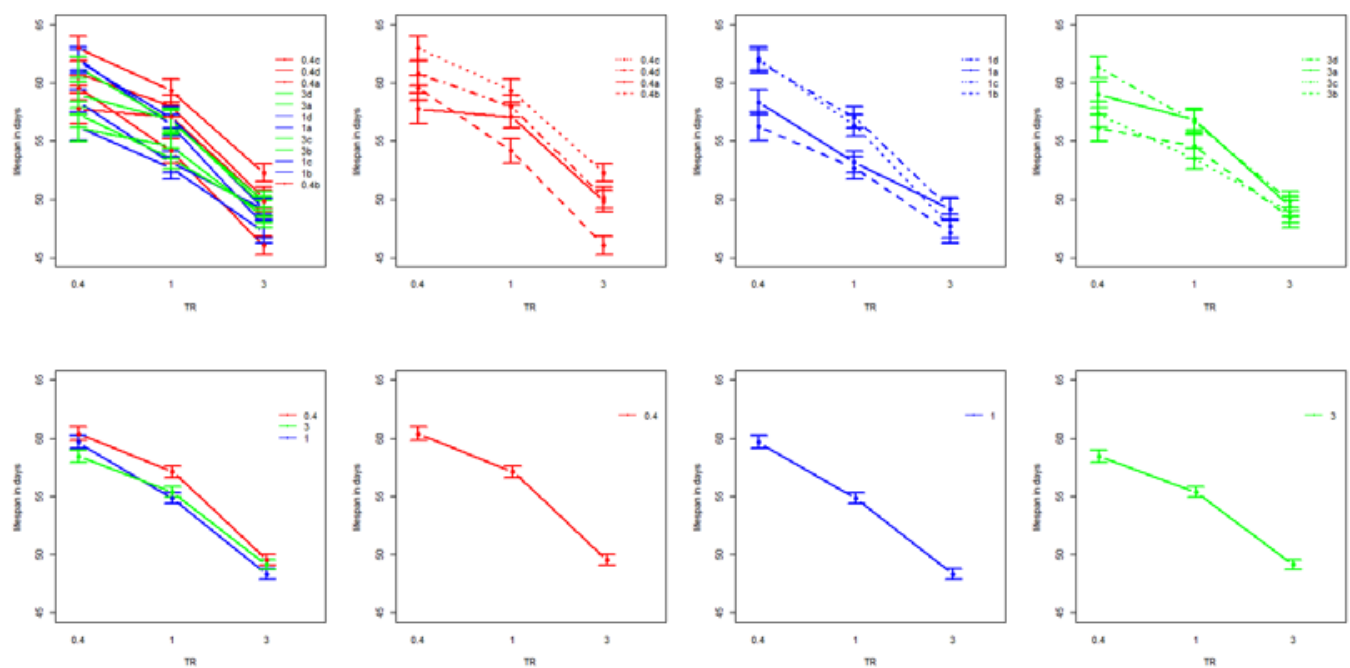

Figure S1: Data from Zajitschek et al. 2016. Nutrient-dependent lifespan effects per population evolved on DR (0.4: red lines), on intermediate food (1: blue lines), and high food (3: green lines). Seemingly consistent effects on lifespan for evolutionary diet reported (lower panel), are rather larger population-specific effects (upper panel). 


\section{Figure S2}

\section{Nutrient-dependent reproduction patterns for different generations}
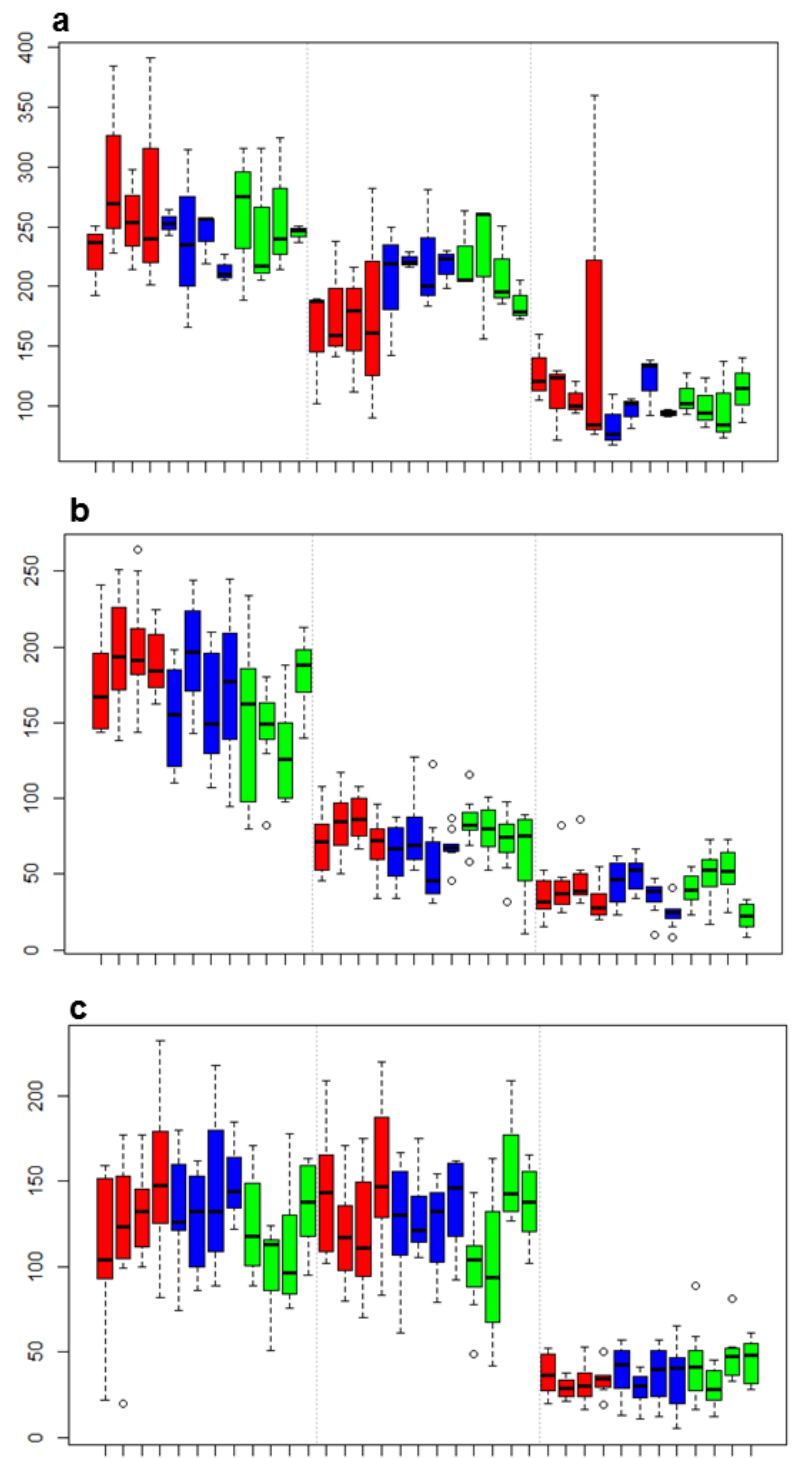

Figure S2: Per population (x-axis) the reproduction effects per population for the response to the three evolutionary food levels, separated by the grey dashed lines. (a) $24^{\text {th }}$ generation (once-mated females, measured on day $6, n=3$ per food type). (b) $42^{\text {nd }}$ generation (continuously-mated females on day $5, n=10$ per food type); (c) $46^{\text {th }}$ generation of continuously-mated females on day $5(n=10)$. Colours represent evolutionary diet conditions: red $=$ low sugar:yeast ratio, blue $=$ intermediate sugar:yeast ratio, and green $=$ high sugar:yeast ratio. 


\section{Figure S3}

\section{Nutrient-dependent lifespan patterns for different generations}
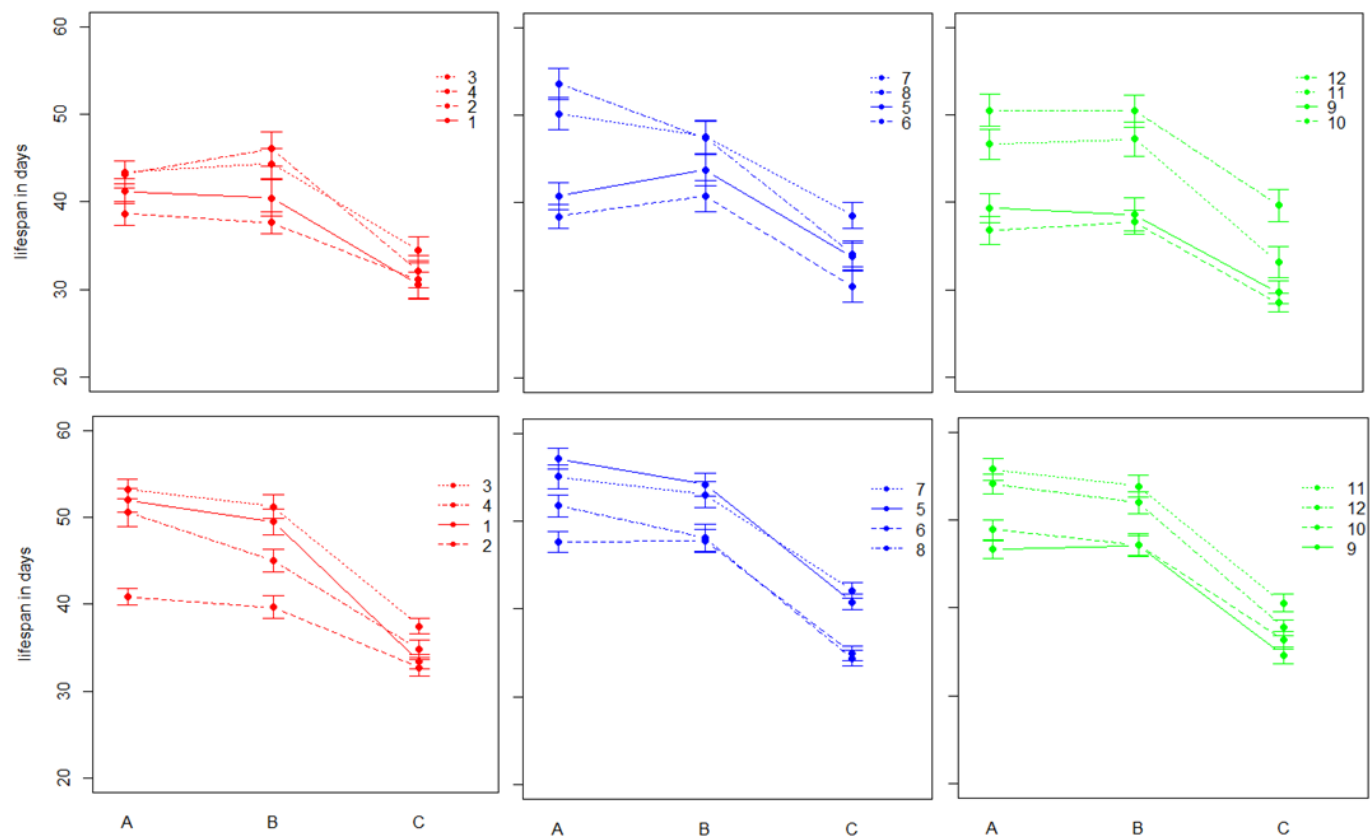

Figure S3: Replicate lifespan trials for the three evolutionary food conditions after (a-c) 24 generations ( $\mathrm{n}=50$ per line per food type) and (d-f) 46 generations of experimental evolution ( $\mathrm{n}=100$ per line per food type). From the 12 populations, two ( 4 and 5) differ in their response to food between the two replicate trials (coxph, $p<0.05$ ), the other 10 lines do not show a different response to the three food types between the two lifespan trials. Colours represent evolutionary diet conditions: red $=$ low sugar:yeast ratio, blue $=$ intermediate sugar:yeast ratio, and green $=$ high sugar:yeast ratio. 


\section{Data S1}

\section{Experimental evolution protocol}

\section{Monday (week 1)}

In the morning, the bottles for the adult treatments will be put in the climate chamber to warm up. Around 12:00/13:00 the newly emerged flies will get their adult food treatment.

Bottles

EvoL 1-4 50S/200Y lowS:Y

EvoI 5-8 100S/100Y intermediateS:Y

EvoH 9-12 200S/50Y high S:Y

The flies stayed in these bottles until Wednesday, after which they will be transferred again.

The bottles for adults have a vertically orientated medium. For the larval stage, I use a normal food orientation (Fig2). The altered food orientation in adults increases surface and thereby reduces adult mortality.

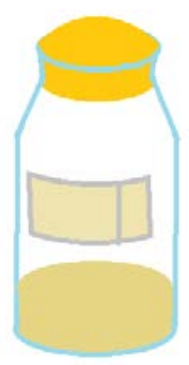

Larval food $100 \mathrm{~S} / 100 \mathrm{Y}$

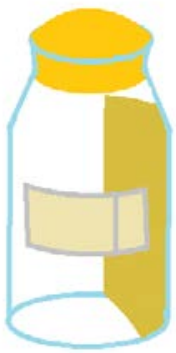

Adult $50 S / 200 Y$

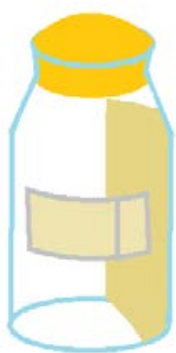

Adult 100S/100Y

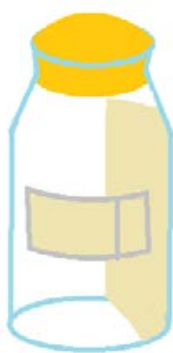

Adult 200S/50Y

Fig 2: Bottles for larval (left) and the three adult food types (right)

\section{Tuesday (week1)}

Nothing happens today, no transfers. Tomorrow (Wednesday again something to do)

\section{Wednesday (week 1)}

New transfer today. In the morning the new bottles will be put in the climate chamber to warm up. Around 12:00 the flies will be transferred to new bottles, on the right adult food type the same as given on Monday. 


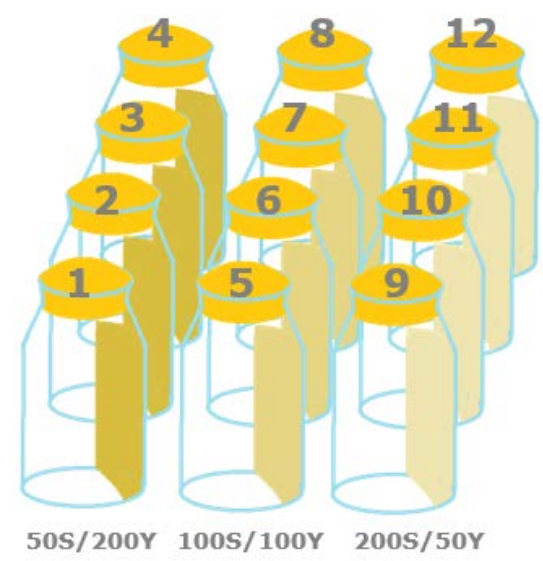

Fig 3: Set up of all replicate population bottles, their numbers and food types.

\section{Thursday (week1)}

Today the flies will be transferred to cages, given the same food as last three days, only now provided on petri dishes (fig 4). Around 11:00 hr the flies should be transferred to cages. For every generation I want them to produce eggs for $26 \mathrm{hrs}$, so that for the next day around 13:00 hr the next generation can be set up with enough eggs but without emerging larvae.
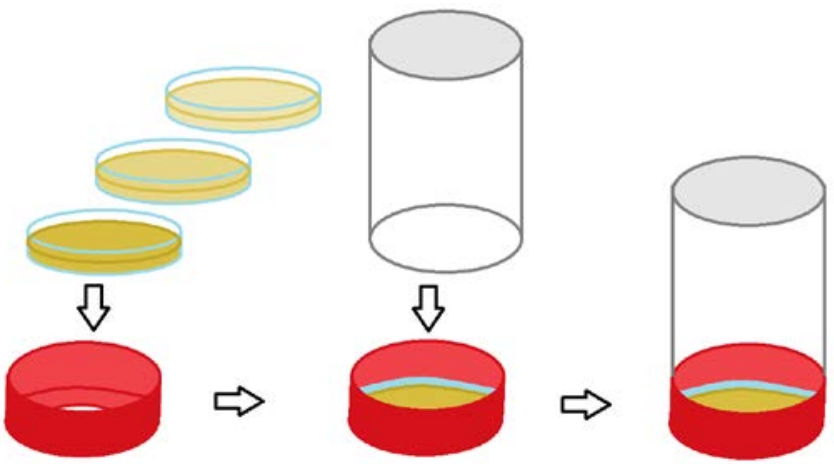

Fig 4: Petri dishes containing the treatment food types will be placed in the red rubbers. These fit onto the cages. (See text for explanation)

To transfer the flies to cages, place a funnel that is big enough to cover an empty cage. After you smashed down the bottle containing flies, remove the plug and transfer the flies quickly to the empty cage by smashing the bottle on the funnel/cage. When the flies are successfully transferred to the cage, quickly place the red rubber with the right food on the cage (fig 5). Remove the label from the bottle to the cage. Do this with all bottles, in total there are 12 population cages to set up. 


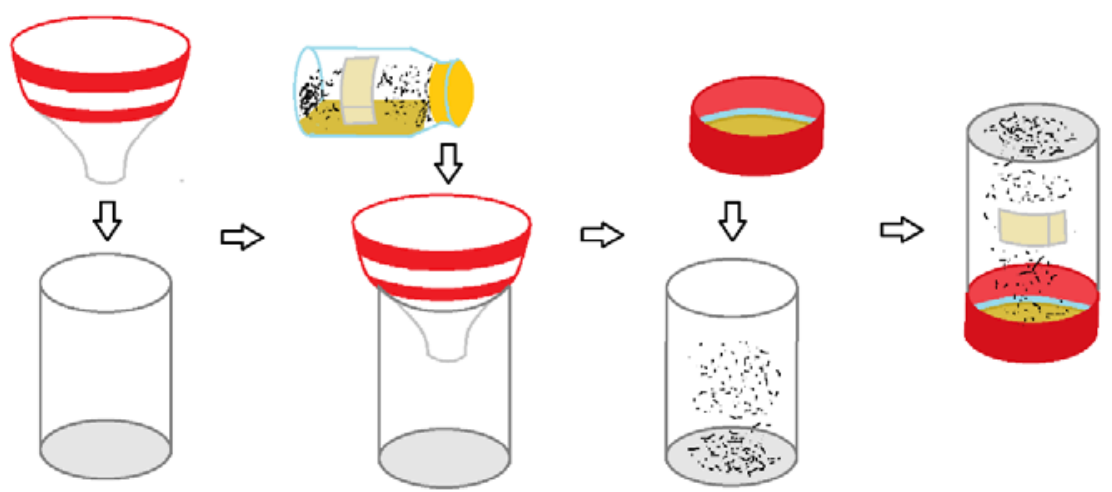

Fig 5: How to transfer flies from bottles to cages. (See text for explanation)

\section{Friday(week1)}

Today the more extensive part of the experiment arrives, an equal amount of eggs of each condition will be transferred to a new bottle for next generation. This process should take about $2 \mathrm{hrs}$.

We start the egg collection $24 \mathrm{hrs}$ after the cages are set up, generally on Friday around 13:00 hr. First, put the cage upside down on a $\mathrm{CO} 2$ pad and wait till the most flies are sedated. Then remove the red rubber and release the Petri dish. Then remove dead flies. Spray $+/-5 \mathrm{ml}$ PBS on the petri dish and loosen the eggs from the food using a brush. Then transfer the PBS/eggs mix to a small centrifuge tube, using an iron funnel. Repeat this step another time for the same petri dish to make sure the most eggs are in the tube. Wash the remaining eggs from the funnel sides with PBS. When done with each replicate, rinse the funnel with water and dry it (especially inside) to make sure no eggs are left.

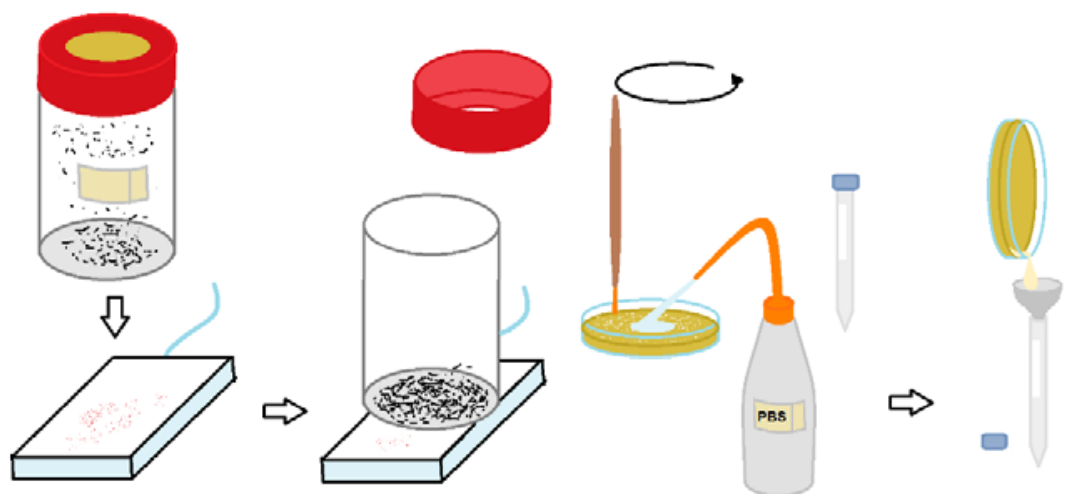

Fig 6: How to transfer all eggs from the petri dish to a centrifuge tube. (See text for explanation)

Do this step for every cage of this treatment (so 4 in total), in the meantime move the centrifuge tubes a couple of times to make sure the majority of eggs have settled at the bottom of the tube. 
After you finished the fourth cage of the treatment, you can remove the supernatant with a big $(5 \mathrm{ml})$ pipet till just above the settled eggs (starting with the first of the four treatments). Then take a yellow pipet, cut it through at $6 \mathrm{~mm}$ and place it on a pipet calibrated at $42 \mathrm{ul}$. Depress the plunger and move the tip just into the mass of eggs and then release the plunger quickly (i.e., let go) to ensure maximum uptake. This should result in a well-packed volume of eggs and consistency can be achieved with only a little practice. If there are air bubbles in the tip, retry it till the sucked up solution is filled with eggs, only then there are enough eggs for a reasonable population size in the next generation. When this goes right, the eggs can be expelled onto the new bottle for fly development (example of bottle in figure 1, left bottle). Then take up $42 \mathrm{ul}$ supernatant PBS from the same tube, but without eggs, and release the content in the same bottle. This is to make sure no eggs are left over in the pipet tip. Shake the bottle a little bit to spread the eggs over the entire surface.

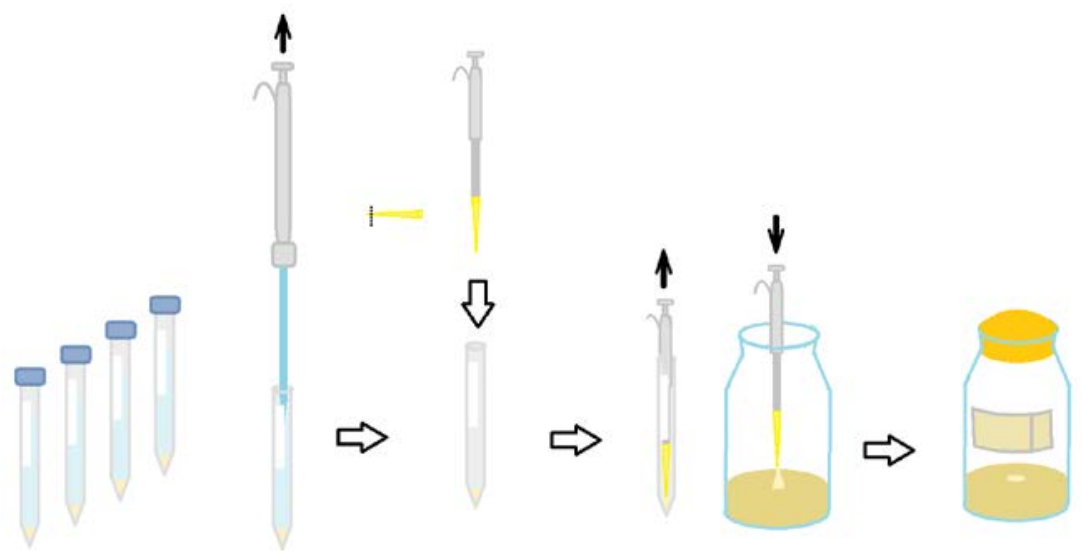

Fig 7: How to transfer and normalize eggs to use for the next generation. (See text for explanation)
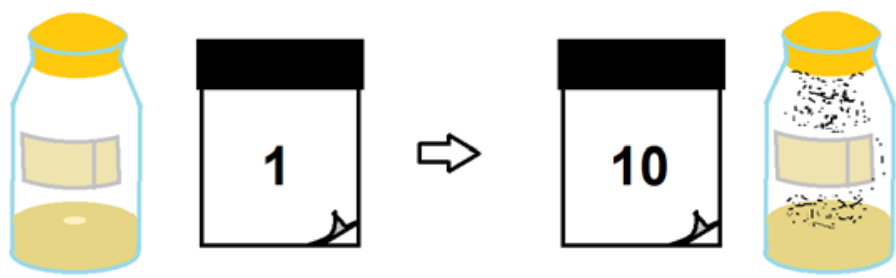

Fig 8: The next generation of flies will now develop under controlled larval density. From egg to adult this takes about 10 days (fig 8 ).

\section{References}

Clancy et al, 2001, Dros. Inf. Serv. 


\section{Table S1}

\section{Overview all assessed phenotypes per generation}

Table S1: Overview of assessed phenotypes per generation, diets tested and the number of biological replicates tested per population.

\begin{tabular}{|c|c|c|c|}
\hline Generation & Phenotype & Diet & $\begin{array}{l}\text { Biological } \\
\text { replicates }\end{array}$ \\
\hline 1 & Reproduction & $3^{*} 3$ sugar $^{\star}$ yeast & $\mathrm{n}=10 \mathrm{p} / \operatorname{diet}$ \\
\hline 1 & $\begin{array}{l}\text { (Male and female) } \\
\text { lifespan }\end{array}$ & $3^{\star} 3$ sugar $^{*}$ yeast & $\mathrm{n}=150 \mathrm{p} /$ diet \\
\hline 24 & Reproduction & $\begin{array}{l}3^{\star} 3 \text { sugar }^{\star} \text { yeast }+ \\
50 \mathrm{~S} / 150 \mathrm{Y}\end{array}$ & $\mathrm{n}=3 \mathrm{p} /$ diet \\
\hline 24 & Lifespan & $\begin{array}{l}3^{*} 3 \text { sugar }^{*} \text { yeast }+ \\
50 \mathrm{~S} / 150 \mathrm{Y}\end{array}$ & $\mathrm{n}=50 \mathrm{p} /$ diet \\
\hline 26 & Body composition: & standard food & $\mathrm{n}=8$ \\
\hline 36 & Starvation resistance & SR food & $\mathrm{n}=120$ \\
\hline 42 & Reproduction & 3 evolutionary. diets & $\mathrm{n}=10 \mathrm{p} /$ diet \\
\hline 42 & Development time & Standard food & $\mathrm{n}=10$ \\
\hline 46 & Reproduction & 3 evolutionary. diets & $\mathrm{n}=10 \mathrm{p} /$ diet \\
\hline 46 & Lifespan & 3 evolutionary. diets & $\mathrm{n}=100 \mathrm{p} / \mathrm{diet}$ \\
\hline
\end{tabular}




\section{Table S2}

\section{Statistical analyses}

Table S2.1: Anova estimates for the effects of dietary sugar and yeast levels and their interaction on reproduction after six days of once-mated females from the ancestral population. ${ }^{*} \mathrm{p}<0.05 ;{ }^{* *} \mathrm{p}<0.01 ;{ }^{* * *} \mathrm{p}<0.001$

\begin{tabular}{lllll}
\hline Factor & Sum Sq & Mean $\mathrm{Sq}$ & F-value & $\operatorname{Pr}(>F)$ \\
\hline sugar & 5.79 & 2.893 & 48.57 & $1.37 \mathrm{e}-14^{* * *}$ \\
yeast & 55.92 & 27.958 & 469.44 & $<2 \mathrm{e}-16^{* * *}$ \\
sugar $^{*}$ yeast & 4.76 & 1.189 & 19.96 & $1.81 \mathrm{e}-11^{* * *}$ \\
\hline
\end{tabular}

Table S2.2: Cox proportional hazard estimates for the effects of dietary sugar (S) and yeast (Y) levels, gender and their interactions on lifespan of once-mated males and females from the ancestral population. ${ }^{*} \mathrm{p}<0.05 ;{ }^{* *}$ $\mathrm{p}<0.01 ;{ }^{* * *} \mathrm{p}<0.001$

\begin{tabular}{|c|c|c|c|}
\hline Factor & Loglik & Chisq & $\operatorname{Pr}(>C h i)$ \\
\hline sugar & -17307 & 352.4667 & $<2.2 \mathrm{e}-16^{* * *}$ \\
\hline yeast & -17248 & 117.4559 & $<2.2 \mathrm{e}-16^{* * *}$ \\
\hline $\operatorname{sex}$ & -17102 & 292.2749 & $<2.2 \mathrm{e}-16^{* * *}$ \\
\hline sugar $^{*}$ yeast & -17100 & 3.9068 & 0.4188 \\
\hline sugar $^{*} \operatorname{sex}$ & -17071 & 58.2447 & $2.25 e-13^{* * *}$ \\
\hline yeast ${ }^{*}$ sex & -17063 & 16.7131 & $0.0002^{* * *}$ \\
\hline sugar $^{*}$ yeast ${ }^{*}$ sex & -17058 & 9.8841 & $0.0424259^{* *}$ \\
\hline
\end{tabular}

Table S2.3: Linear mixed effect model estimates for the effects of evolutionary food (A, B or C) and evolutionary treatments (evoL, evoI or evoH) and their interaction on early reproduction. Analysis is performed on combined reproduction data of all assessed generations $(24,42$ and 46$)$, by using "generation" as a random factor. ${ }^{\star} \mathrm{p}<0.05 ;{ }^{* *} \mathrm{p}<0.01 ;{ }^{* *} \mathrm{p}<0.001$

\begin{tabular}{lllll}
\hline Factor & Estimate & Std. Error & T-value & $\operatorname{Pr}(>|t|)$ \\
\hline Intercept & 67.921 & 4.066 & 16.705 & $<2 \mathrm{e}-16^{* * *}$ \\
$B$ & -64.190 & 5.750 & -11.163 & $<2 \mathrm{e}-16^{* * *}$ \\
$C$ & -127.917 & 5.750 & -22.246 & $<2 \mathrm{e}-16^{* * *}$ \\
evoI & -9.167 & 5.750 & -1.594 & 0.11132 \\
evoH & -23.155 & 5.750 & -4.027 & $6.23 \mathrm{e}-05^{* * *}$ \\
$B^{*}$ evoI & 8.190 & 8.132 & 1.007 & 0.31416 \\
$C^{*}$ evoI & 6.048 & 8.132 & 0.744 & 0.45729 \\
$B^{*}$ evoH & 23.119 & 8.132 & 2.843 & $0.00459^{* *}$ \\
$C^{*}$ evoH & 24.643 & 8.132 & 3.030 & $0.00253^{* *}$ \\
\hline
\end{tabular}


Table S2.4: Mixed-effect cox proportional hazard estimates for the effects of dietary sugar (S100, S200) and yeast (Y100,Y200) levels, evolutionary treatment and interactions on lifespan of once-mated females of the $24^{\text {th }}$ generation using population as random factor.

\begin{tabular}{llllll}
\hline & coef & exp(coef) & se(coef) & $z$ & $p$ \\
& & & & & \\
\hline & & & & & \\
F1S100 & 0.277220936 & 1.3194579 & 0.1029511 & 2.69 & 0.0071 \\
F1S200 & 1.084499503 & 2.9579590 & 0.1073793 & 10.10 & 0.0000 \\
F1Y100 & -0.113923557 & 0.8923262 & 0.1025817 & -1.11 & 0.2700 \\
F1Y200 & 0.295319367 & 1.3435554 & 0.1021189 & 2.89 & 0.0038 \\
F1evoI & -0.425452046 & 0.6534743 & 0.2475308 & -1.72 & 0.0860 \\
F1evoH & -0.150826097 & 0.8599972 & 0.2473066 & -0.61 & 0.5400 \\
F1S100:F1Y100 & -0.047486418 & 0.9536234 & 0.1457700 & -0.33 & 0.7400 \\
F1S200:F1Y100 & 0.171583039 & 1.1871827 & 0.1482839 & 1.16 & 0.2500 \\
F1S100:F1Y200 & -0.202465152 & 0.8167149 & 0.1448947 & -1.40 & 0.1600 \\
F1S200:F1Y200 & -0.162038364 & 0.8504086 & 0.1474273 & -1.10 & 0.2700 \\
F1S100:F1evoI & 0.047063346 & 1.0481884 & 0.1448740 & 0.32 & 0.7500 \\
F1S200:F1evoI & 0.171817241 & 1.1874608 & 0.1500945 & 1.14 & 0.2500 \\
F1S100:F1evoH & -0.217123642 & 0.8048305 & 0.1449919 & -1.50 & 0.1300 \\
F1S200:F1evoH & 0.091438306 & 1.0957492 & 0.1523572 & 0.60 & 0.5500 \\
F1Y100:F1evoI & 0.113773154 & 1.1204979 & 0.1457053 & 0.78 & 0.4300 \\
F1Y200:F1evoI & 0.037398967 & 1.0381071 & 0.1442539 & 0.26 & 0.8000 \\
F1Y100:F1evoH & -0.006923339 & 0.9931006 & 0.1449980 & -0.05 & 0.9600 \\
F1Y200:F1evoH & -0.072692406 & 0.9298868 & 0.1440609 & -0.50 & 0.6100 \\
F1S100:F1Y100:F1evoI & 0.038834030 & 1.0395979 & 0.2060965 & 0.19 & 0.8500 \\
F1S200:F1Y100:F1evoI & -0.388158372 & 0.6783049 & 0.2085978 & -1.86 & 0.0630 \\
F1S100:F1Y200:F1evoI & 0.022340936 & 1.0225924 & 0.2039769 & 0.11 & 0.9100 \\
F1S200:F1Y200:F1evoI & -0.296453815 & 0.7434500 & 0.2072315 & -1.43 & 0.1500 \\
F1S100:F1Y100:F1evoH & 0.289028913 & 1.3351303 & 0.2056689 & 1.41 & 0.1600 \\
F1S200:F1Y100:F1evoH & -0.212999891 & 0.8081562 & 0.2100500 & -1.01 & 0.3100 \\
F1S100:F1Y200:F1evoH & 0.221439144 & 1.2478713 & 0.2041600 & 1.08 & 0.2800 \\
F1S200:F1Y200:F1evoH & -0.217562714 & 0.8044772 & 0.2090229 & -1.04 & 0.3000 \\
& & & & & \\
\hline & & & & &
\end{tabular}


Table S2.5: Cox proportional hazard estimates for the effects of dietary sugar and yeast levels, population and interactions on lifespan of once-mated females of the $24^{\text {th }}$ generation. ${ }^{*} \mathrm{p}<0.05 ;{ }^{* *} \mathrm{p}<0.01 ;{ }^{* * *} \mathrm{p}<0.001$

\begin{tabular}{|c|c|c|c|}
\hline Factor & loglik & Chisq & $\operatorname{Pr}(>|C h i|)$ \\
\hline sugar & -38393 & 27.119 & $1.292 \mathrm{e}-06^{* * *}$ \\
\hline yeast & -38112 & 563.248 & $<2.2 \mathrm{e}-16^{\star * *}$ \\
\hline population & -38101 & 20.678 & $0.0004^{\star * *}$ \\
\hline sugar ${ }^{\star}$ population & -38084 & 34.111 & $0.0479 *$ \\
\hline yeast ${ }^{*}$ population & -38061 & 47.794 & $0.0012^{* *}$ \\
\hline sugar ${ }^{\star}$ yeast ${ }^{*}$ population & -38027 & 66.162 & 0.0170 * \\
\hline
\end{tabular}

Table S2.6: Cox proportional hazard estimates for the effects of population and evolutionary diet on starvation resistance of females in the $36^{\text {th }}$ generation. ${ }^{*} \mathrm{p}<0.05 ;{ }^{* *} \mathrm{p}<0.01 ;{ }^{* * *} \mathrm{p}<0.001$

\begin{tabular}{llll}
\hline Factor & loglik & Chisq & $\operatorname{Pr}(>\mid$ Chi $\mid)$ \\
\hline evolution & -9166.8 & 2.0861 & 0.3524 \\
population & -9137.3 & 61.073 & $5.856 \mathrm{e}-09^{* * *}$ \\
\hline
\end{tabular}




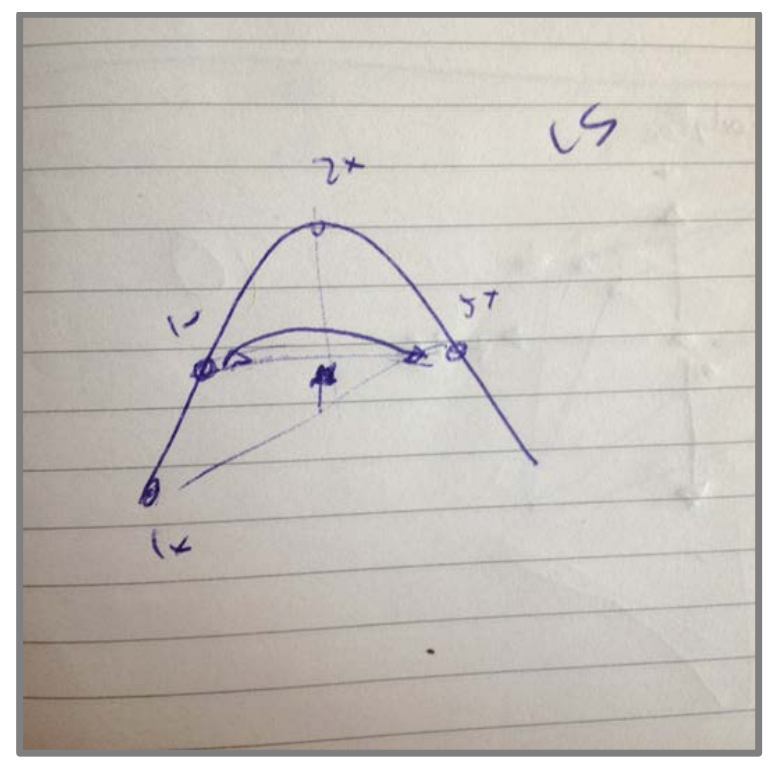

Lifespan by diet reaction norm and yoyo behavior 


\section{CHAPTER VI}

\section{PERVASIVE GENE EXPRESSION RESPONSES TO A}

\section{FLUCTUATING DIET IN DROSOPHILA MELANOGASTER}

The importance of measuring multiple traits to decouple potential mediators of lifespan and reproduction

Jelle Zandveld*

Joost van den Heuvel ${ }^{\star}$

Maarten Mulder

Paul M. Brakefield

Thomas B. L. Kirkwood

Daryl P. Shanley

Bas J. Zwaan

* Shared first author

Chapter published in: Evolution 


\section{ABSTRACT}

Phenotypic plasticity is an important concept in life history evolution, and most organisms, including Drosophila melanogaster, show a plastic life-history response to diet. However, little is known about how these life-history responses are mediated. In this study, we compared flies fed an alternating diet (yoyo flies) with flies fed a constant low $(\mathrm{CL})$ or high $(\mathrm{CH})$ diet and tested how whole genome expression was affected by these diet regimes and how the transcriptional responses related to different life history traits. We showed that flies were able to respond quickly to diet fluctuations throughout lifespan by drastically changing their transcription. Importantly, by measuring the response of multiple life history traits we were able to decouple groups of genes associated with lifespan or reproduction, life-history traits that often co-vary with a diet change. A co-expression network analysis uncovered which genes underpin the separate and shared regulation of these lifehistory traits. Our study provides essential insights to help unravel the genetic architecture mediating life history responses to diet, and it shows that the flies' whole genome transcription response is highly plastic. 


\section{INTRODUCTION}

Phenotypic plasticity, the ability of a genotype to produce alternative phenotypes in different environments (Piersma \& Drent, 2003; West-Eberhard, 2003), is an important concept in the study of life history evolution (Flatt \& Heyland 2011; Stearns, 1992). Life history traits can be greatly influenced by biotic factors such as competition (Maret \& Collins, 1997), as well as by abiotic factors such as temperature (Marshall \& Sinclair, 2009) and nutrient availability (Bento et al. 2010; May et al. 2015). Such plastic responses, which may be subtle and continuous in nature, can be studied in detail in controlled laboratory experiments. For instance, in many species mild dietary restriction, a reduction of food intake without malnutrition, increases lifespan (Chippindale et al. 1993; Klass 1977; McCay et al. 1935; Muller et al. 1980; Weindruch et al. 1986). Other life-history characteristics also vary with differential dietary intake, such as growth (McCay et al. 1935, Weindruch 1986), reproduction (Emran et al. 2014, Van den Heuvel et al. 2014, Solon-Biet et al. 2015), and body composition (Skorupa et al. 2008; Solon-Biet et al. 2013). In Drosophila melanogaster phenotypes for many life history traits are affected by diet, including lifespan, reproduction, weight, fat content, starvation resistance, with a typical negative correlation between lifespan and (early) reproduction (Flatt, 2011; Piper \& Partridge, 2007; Skorupa et al. 2008; Emran et al. 2014, but also see Grandison et al. 2009, Van den Heuvel et al. 2017). Moreover, when switching between diets during adult life, the fly's life history phenotype can change dramatically as well. For instance, a switch from a high to a low dietary level can reduce mortality rate of flies within 48 hours (Mair et al. 2003), and also affect egg production (Good \& Tatar, 2001). Since many species experience a dynamically changing diet exposure in nature, we decided that measuring effects of (repeated) diet changes during life in the laboratory might better reflect the natural environment than testing the effects of a constant diet.

In a previous study we showed that flies transferred between two diets alternate their reproduction pattern within a few days to a level comparable to the differences between flies that were fed a constant diet (Van den Heuvel et al, 2014; Figure1). The magnitude and speed of the response suggests a mechanism of plasticity that maximizes fitness in a fluctuating environment. Theoretical models aimed at explaining empirical phenotypic data on reproduction and lifespan have shown that such phenotypic plasticity can be adaptive (Shanley and Kirkwood 2000; Mangel 2001). However, such an approach cannot by itself uncover the mechanistic underpinning of this life history response.

Functional analyses of genes mediating the flies' life-history response have been performed in flies, and many genetic or dietary interventions (e.g. in the insulin signaling or target of rapamycin, ToR, pathway) are known to affect life-history traits such as lifespan, reproduction, development time, and fat content (Gronke et al. 2010; Grandison et al. 2009; Emran et al. 2014). Measuring gene expression is a common way to analyze whether genes are related to phenotypes that respond plastically to diet change. Quantification of the whole genome expression of flies has led to gene 
sets that correlate to effects on lifespan either in constant low or high diets (Pletcher et al. 2002; Doroszuk et al. 2008; Bauer et al. 2011), or following a single change in diet (Gershman et al. 2007; Whitaker et al. 2014). However, there is a need also to consider a multiple changing diet which may be more evolutionarily relevant but which is for far untested in whole genome transcription studies. In addition, many gene expression studies primarily focus on the effects of diet on lifespan; they do not include analyses of other phenotypes. For example, reproduction typically correlates with the same diet change, and is often enriched in gene ontology analyses (Pletcher et al. 2002; Doroszuk et al. 2010; Gershman et al. 2007), but measured scarcely. Furthermore, using treatments for which reproduction and lifespan do not co-vary are especially helpful to parse apart the diet effects on the individual life history traits (Grandison et al. 2009; Antosh et al. 2011). Indeed, in our previous work we showed that flies experiencing a fluctuating diet throughout life alter reproduction very quickly, but live longer than control flies on constant low diet (Van den Heuvel et al. 2014), thus in principle allowing for the separation of gene expression patterns that relate to lifespan or reproduction.

In this study, using the same experimental fluctuation dietary conditions of our previous work, we set out to test, (1) whether variation in nutrition causes variations in whole genome expression, (2) how the transcriptome of flies on a fluctuating diet compares to that of flies on constant food, and, (3) how the transcriptome relates to direct effects of diet and to reproduction and lifespan phenotypes.

\section{METHODS}

Diet

We used three different nutritional environments, indicated by $1 \mathrm{x}$ (low), $2 \mathrm{x}$ (intermediate) and $5 \mathrm{x}$ (high) medium. The nutritional environments varied in sugar (50, 100 and 250 gram/liter in 1x, $2 \mathrm{x}$ and $5 \mathrm{x}$ medium, respectively) and yeast $(35,70,175$ gram/liter in $1 \mathrm{x}, 2 \mathrm{x}$ and $5 \mathrm{x}$ medium, respectively). Furthermore, each nutritional environment contained the same concentration of agar (20 gram per liter), nipagine (15 $\mathrm{ml}$ of $100 \mathrm{~g}$ 4-methyl hydroxy benzoate per liter alcohol), and propionic acid (3 $\mathrm{ml}$ per liter).

\section{Fly stock and husbandry}

Flies were collected in the summer of 2008 at six locations along a transect ranging from Vienna to Athens. The flies were mixed and reared in population bottles with population numbers of at least 300 individuals per generation on $1 \mathrm{x}$ medium for 50 generations before the experiments were started (Van den Heuvel et al. 2014; May et al. 2015). To prevent larval and maternal effects on adults, we kept the flies for at least three generations on $2 \mathrm{x}$ food. The larvae were reared in vials with $6 \mathrm{ml}$ of $2 \mathrm{x}$ food, with a density of 50 eggs per vial. Without using anesthesia, after eclosion each fly was randomly assigned to a $23 \mathrm{ml}$ vial either containing $6 \mathrm{ml} \mathrm{1x}$ or $5 \mathrm{x}$ food. For the first 
three days, the singly housed flies were all checked for sex and fertilized eggs; males and fertilized females were removed from the experiment. Therefore, all flies in this experiment were singly housed virgin female flies.

\section{Adult treatments}

Four adult diet treatments were used (see Figure S1) and for each treatment we set up 65 flies. One group of flies was maintained on low diet throughout the experiment (low control, CL). A second group of flies was maintained on high diet throughout the experiment (high control, $\mathrm{CH}$ ). A third group was transferred between vials of high and low diet every half week. Because the initial vial of this group of flies contained high diet, we call this group the yoyo high-start line (Yh). A fourth treatment group started at the low diet medium (yoyo low-start line, Yl), to control for the potential effect of first diet resource level. To keep in synchrony with the week and make sure that both yoyo treatments in total had the same number of days on each nutrition level throughout the experiment, flies were transferred every three or four days.

\section{Phenotypic measurements}

Lifespan and the age-related reproduction patterns of all diets were adapted from our previous study (see Van den Heuvel et al. 2014 for all the measurements). For the current study, in which we measured gene expression of the exact same treatments, we recorded the number of eggs laid by each female on day 34 or day 38 after eclosion, the day before sacrifice for the expression analyses.

\section{Microarray experiment}

For the microarray experiment a new cohort of flies was set up. The flies received the same treatment as those for life history assessment, with the exception that after being anesthetized, they were not weighed. Flies were sacrificed at two time points in the experiment: at the age of 34 and 38 days (Figure S1). We did this in order to be able to compare the Yl and Yh treatment flies properly (i.e. to correct for initial diet and potential time effects). We sacrificed all the flies of the gene expression assay at similar times during the day (at noon) and for all groups the flies had spent four days in their food vial. We transferred flies to $2 \mathrm{ml}$ Eppendorf tubes (4 flies per tube) and snap froze them using liquid nitrogen. After this, flies were kept at -80 degrees Celsius before RNA isolation. RNA was isolated using the TRIZOL method after which we used the RNAeasy from Quiagen to clean up the samples. For both steps manufactures' protocols were used. All 32 samples passed quality control and microarray measurements took place at Service XS in Leiden, the Netherlands (http://www.servicexs.com/) using the Affymetrix Drosophila genome 2.0 array.

\section{Statistics}

For the reproduction data we used a GLM with a Poisson error distribution. The microarray data were normalized and summarized using the robust multi-array average (RMA) procedure (Bolstad 
et al. 2003), as implemented in Bioconductor, R (Gentleman et al. 2005; Hahne et al. 2008). We used MAANOVA to calculate permutated and adjusted FDR values using Fs test statistics and used a cutoff value of 0.05 to call a difference significant. Gene expression was also correlated (Pearson) with number of eggs per sample, where again the p-values were FDR adjusted.

\section{Meta-analysis}

We compared our results to three other studies that used a technically similar setup (female Drosophila melanogaster flies, whole bodies, Genome 2.0 Affymetrix microarray) and that were designed to study the effect of nutritional and/or reproduction interventions on lifespan. Doroszuk et al. (2012) kept flies on different adult food conditions and measured transcription of flies at middle (90\% alive) and old (10\% alive) age for control and starvation resistance selected lines (the latter also showed increased longevity; Doroszuk et al. 2012). Bauer et al. (2010) used both genetic manipulation and dietary restriction to increase lifespan of flies and measured whole genome transcription of flies at young (10 days) and middle (30 days, roughly $90 \%$ alive) age (Bauer et al. 2010). Yamamoto et al. (2013) measured gene expression of flies on day 14 for which the corpus allata was ablated and/or for sterile ovoD flies (Yamamoto et al. 2013). We retrieved normalized data from the gene omnibus and we fitted a full factorial ANOVA where possible (three different ones for Bauer et al (2010)). We tested whether probe sets were significant in all three data sets omitting age-related changes that did not show interactions with the lifespan extending treatments. This resulted in a probe set list consistent with a lifespan increase in all three studies and was used to compare with the gene lists obtained from this study.

\section{Network analysis}

To establish a co-expression matrix, pairwise Pearson correlation coefficients over the 32 samples were calculated. Using the igraph package we built a network from all genes connected with a cutoff correlation coefficient of 0.95 . Subsequently, we defined modules from the total network when subnetworks were not connected, after which we analyzed the modules that were larger than 20 genes. First, we performed separate PCAs on the subset of probe sets for every module. To functionally link the individual modules to phenotypic responses, we quantified the variation of the samples dependent on the nutrition level (high vs low) and yoyo status (control vs yoyo) for the gene loadings that explained most of the variation between treatments (PC1). The most connected genes in the module were named 'hubs' and identified by taking the genes with more connections than the $99 \%$ quantile value of the connection distribution. Information on these 'hubs' was gathered using Flybase. 


\section{RESULTS}

Phenotypes

In our previous study testing the effects of a yoyo diet on fly lifespan (Van den Heuvel et al. 2014; Figure 1b.), we showed for different trials that flies on a constant high diet $(\mathrm{CH})$ were longer lived compared to flies on a constant low diet (CL), but that yoyo treatments had a considerably longer lifespan then CL flies. Compared to $\mathrm{CH}$ flies only the yoyo flies that started on high food (Yh) had a shorter lifespan (Van den Heuvel et al. 2014; Figure 1b.). Reproduction of yoyo flies varied with every diet switch, increasing on low food and decreasing when switched to high food, resembling the effects of the control diets were compared to $\mathrm{CH}$ flies an increased reproduction was observed for CL flies (Van den Heuvel et al. 2014, Figure 1a.). Here, for our gene expression cohort we counted the number of eggs produced after the last transfer before flies were sacrificed for RNAextraction (Figure S2). This cohort showed very similar effects as previously seen in Figure 1, i.e. the yoyo treatments flies produced more eggs on low food and less when switched to high food (glm, $\mathrm{p}<0.01$, Figure S2), while flies on constant low food (CL) laid more eggs than flies on constant high food $(\mathrm{CH})$ (glm, $\mathrm{p}<0.001$, Figure S2).
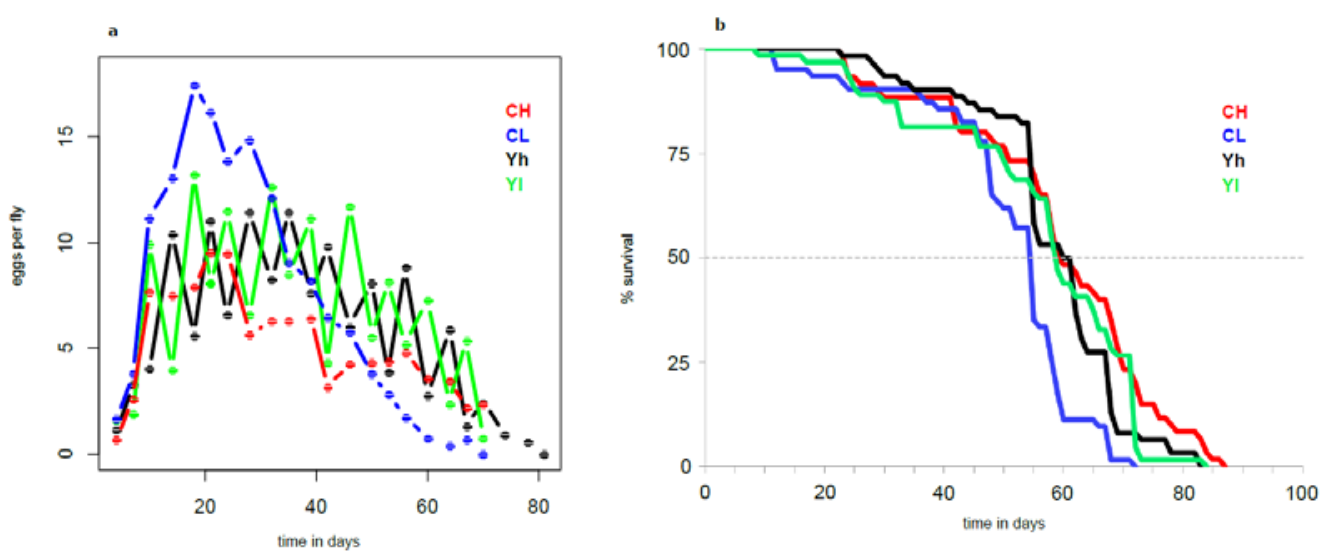

Figure 1. Previously reported effects of a fluctuating diet on reproduction and lifespan (from: Van den Heuvel et al. 2014) (a) Age-dependent reproduction pattern per fly per day for the four different diet treatments, and (b) the proportion of flies alive (\% survival) per day for the same four treatments. The cross point of the treatments with the dashed line represent the median survival per line. Colors correspond with treatment: red for control high $(\mathrm{CH})$, blue for control low $(\mathrm{CL})$, black for high start yoyo (Yh), and green for low start yoyo flies (Yl). 


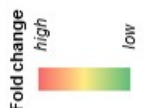
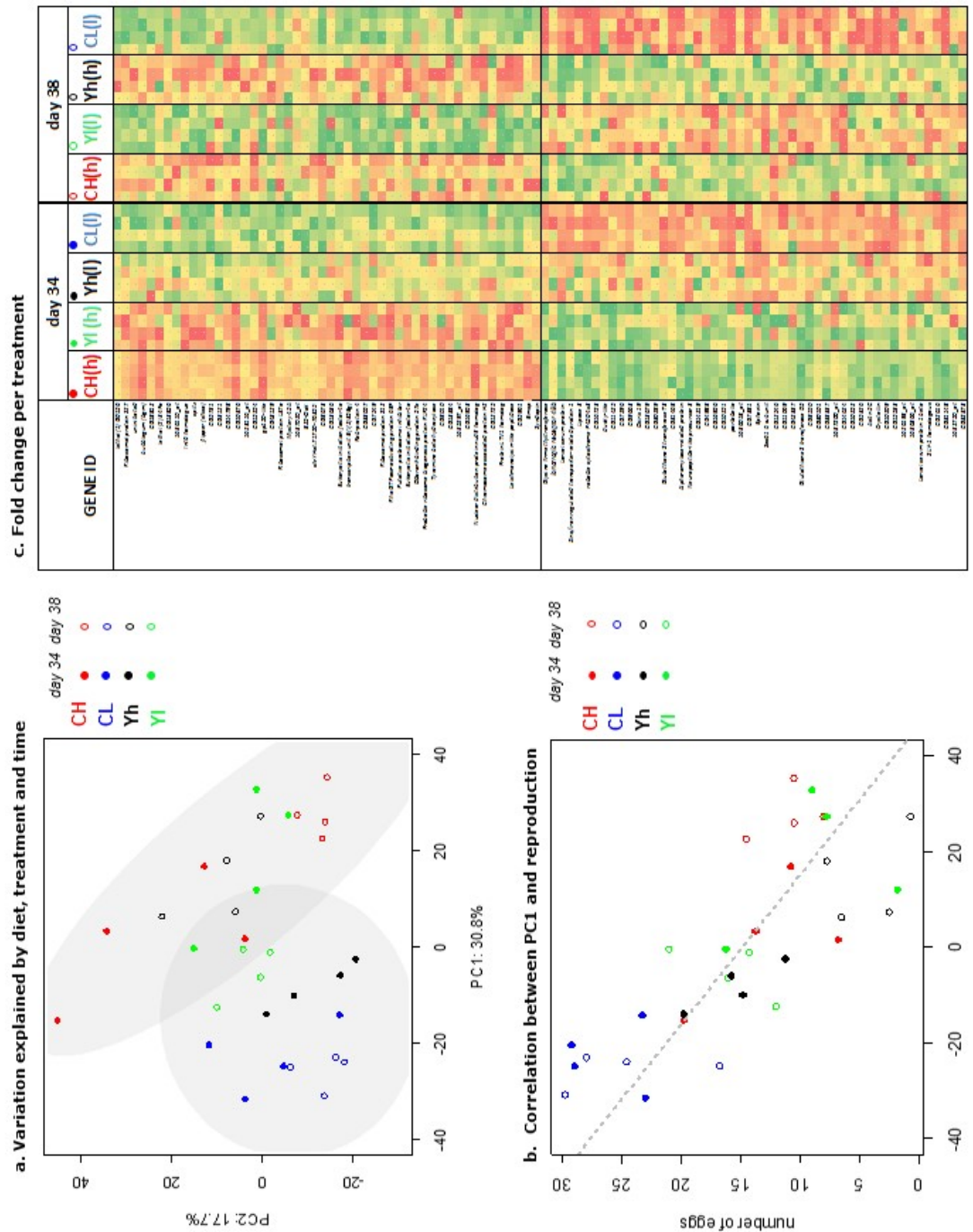

던 பㄷㅀ
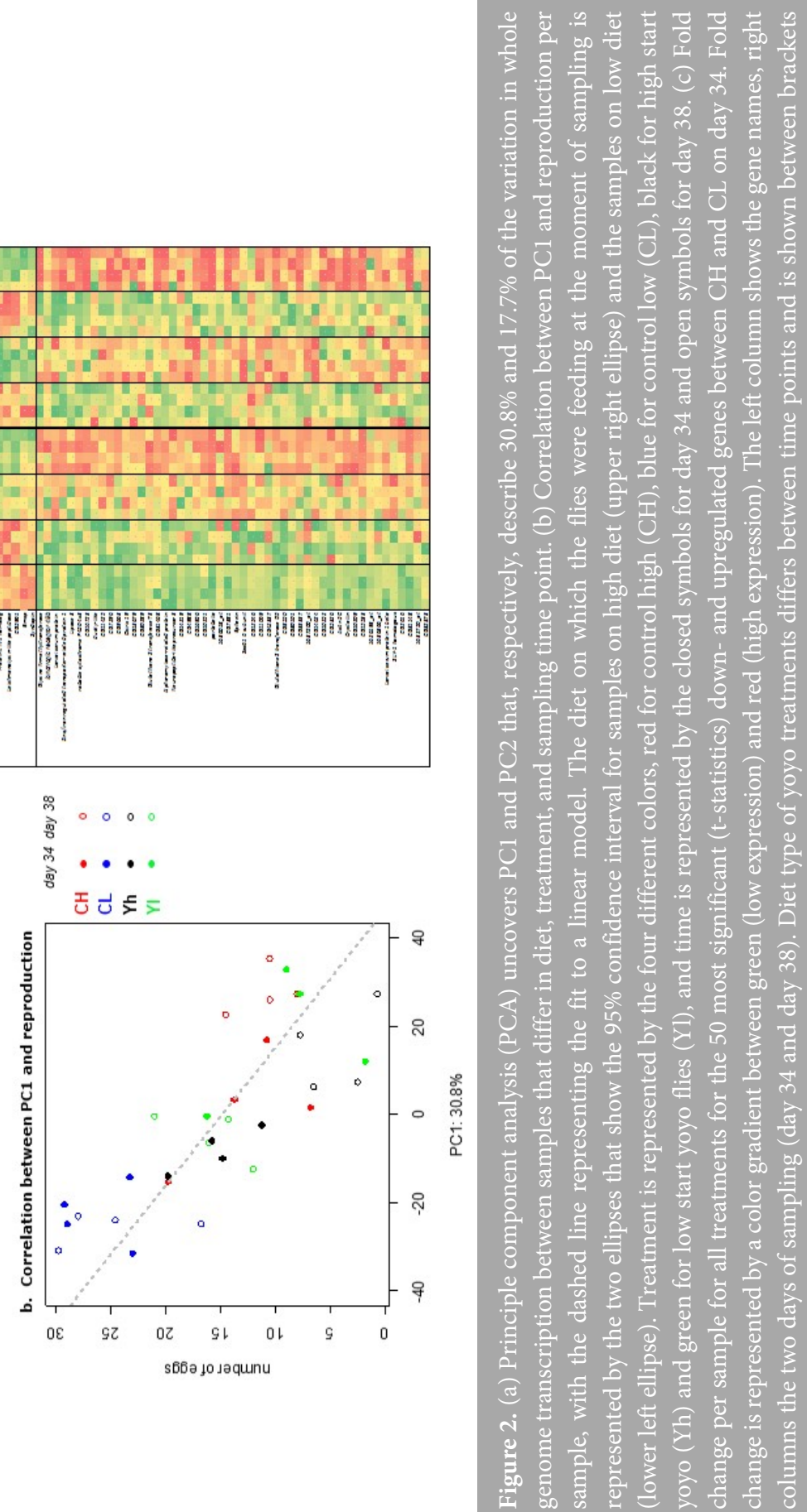


\section{Transcription responses}

In order to test the extent to which the nutritional environment affects the flies' whole genome transcription, we performed a microarray analysis on control and yoyo flies on day 34 and day 38 after eclosion.

Variations in whole genome expression is largely explained by variation in nutrition

Our data indicated that diet affected a very large number of probe sets; when all samples were grouped together, 3150 probe sets were significantly upregulated and 2796 were downregulated on high compared to low diet (MAANOVA, $\mathrm{p}<0.05$, Table $\mathrm{S} 1$ ). When performing a principal component analysis (PCA) on all 32 samples we showed that the first principal component accounts for $30.8 \%$ and the second for $17.7 \%$ of the variation, and that samples grouped well together using diet as the contrasting factor (Figure 2a, ellipses). Moreover, when comparing only the control flies (CL and CH treatments), we see a similar effect; on high food 2341 probe sets were upregulated and 1573 significantly downregulated, resulting in two clearly separated groups for the principle component analysis (Figure 2a, Figure S3). This shows that a very large part of the transcriptome is responding to diet at the moment of sampling only.

\section{Similar transcription response to diet for yoyo flies and flies fed a constant diet}

For the diet effects of the yoyo cohorts (yoyo on high food versus yoyo on low food) 1490 probe sets were upregulated and 1232 downregulated (Table S1). Between this probe set list and that of the controls we found for up- and down-regulation on high diet 989 and 657 overlapping probe sets respectively (Figure 3, Figure S4). These results were reflected in the PCA as samples of yoyo flies for both low and high diet grouped well together with the controls (Figure 2a), again also reflected in four trials of 100 randomly selected probe sets (Figure S3). Consistent with these results, for the 50 most up- and down-regulated genes we observed that for both days the expression change with diet in yoyo flies had a similar pattern to the effects observed in controls (Figure 2c). Taken together, these results demonstrate that when flies change either to a low or high diet, a substantial change of genome transcription occurs, moving strongly towards the transcription signature of flies on the corresponding constant diet.

Specific yoyo effects: mainly on low food and no effect of starting diet.

In addition to the observed general diet effects, we also tested yoyo-specific effects on whole genome transcription; 26 and 48 probe sets were respectively up- and down-regulated with yoyo treatment (MAANOVA, $\mathrm{p}<0.05$, Table S1). On low diet, yoyo flies had a similar orientation to controls in the PCA but were clearly separated, which was not the case on a high diet as the yoyo flies' transcription pattern on high diet did not differ from $\mathrm{CH}$ flies (Figure 2a). These transcription results between control and yoyo flies were consistent with the observed lifespan effects; i.e. yoyo flies lived longer than CL but did not differ from CH flies (Figure 1b). When assessing the number of genes that are associated with the effects of yoyo treatment per diet, indeed 747 genes were 
differentially expressed (483 up, 264 downregulated) between CL and yoyo flies on low food, but none on high food (table S1).

Flies from the two different yoyo treatments were very comparable in both their survival and reproduction responses. Therefore, small gene expression differences were expected between the two groups of flies. Indeed, no probe sets were differentially expressed between yoyo flies that started on high ( $\mathrm{Yh}$ ) compared to those that started on low food (Yl) and also no differentially expressed probe sets were found between these samples when only the high or low diet samples for these Yh and Yl treatments were compared (Table S1).

Time affected whole genome transcription pattern of high control flies, but not in yoyo flies Although the two time points were separated only by four days, the effects of time were clearly visible. When all samples were included, 2848 probe sets were down-regulated and 1576 upregulated between the two time points (Table S1). Especially in control samples the time effect was apparent, as 1826 and 3425 probe sets were respectively up- and down-regulated with time. No such effects, however, were measured in the yoyo flies; no probe sets were either up- or downregulated with time (Table $\mathrm{S} 1$ ).

Testing the control treatments separately, we also observed clear differences in the effect of time; in $\mathrm{CH}$ flies 1643 and 2919 probe sets were respectively up- and down-regulated with time, while in CL flies no probe sets were significantly affected (Table S1). This is in accordance with the PCA for which we observed that time indeed had its largest effects in $\mathrm{CH}$ flies; the samples of the two time points could be distinguished along PC2 but the distance along this axis is much larger for $\mathrm{CH}$ than for CL (Figure 2a).

\section{Parsing apart genes involved in reproduction, diet, and lifespan}

We observed that the diet responses of both the whole genome transcription and egg laying were highly plastic, and to investigate a possible relationship between these two observations, we related the number of eggs per sample with the gene expression per probe set. The first principal component for the gene expression dataset, (explaining $30.8 \%$ of the variation) related remarkably well to egg production per sample (linear model, $\mathrm{R}^{2}=0.61, \mathrm{p}<0.001$, Figure $2 \mathrm{~b}$ ). Indeed, in total 4386 probe sets correlated with egg number; 2433 were negatively correlated with egg number and 1953 probe sets positive. This correlation suggests that probe sets associated to the treatment effects largely overlap with the probe set list that correlates with egg laying. The probe sets that differ between treatments but do not overlap with this 'reproduction list' are thus likely to be involved in processes that differ between the treatments other than reproduction, for instance nutrient sensing and/or lifespan. Our set-up allowed us to address which probe sets were associated with each of these processes. 
The two control conditions (CH and CL) differ in diet, lifespan, and reproduction (Figure 1). Of the 3914 probe sets that differ between these two treatments, 960 probe sets were not associated with reproduction (Figure $3 \mathrm{a}$ ) and more probably related to the differences in diet or lifespan between the $\mathrm{CH}$ and CL flies. CL and yoyo flies also showed lifespan and reproduction differences, but had the same diet when yoyo flies were on low food. For this comparison we found 747 probe sets to be differently expressed; these transcriptional differences are not explained by diet differences as for both conditions diet was the same, but rather by factors that do differ between the treatments, such as lifespan and reproduction. From these 747 probe sets, 137 had an overlap with the 960 probe sets that were not associated with reproduction between CH and CL (Figure 3a). These 137 are thus more likely to be candidates for the lifespan differences between the treatments, or are associated with other unmeasured phenotypes that correlate with our treatment effects on lifespan. Lastly, the probe sets associated to reproduction, those differentially expressed between $\mathrm{CH}$ and CL and between CL and yoyo on low food, showed an overlap of 443 probe sets. This list is therefore representative for the reproduction differences between the treatments.

Meta-analysis reveals substantial overlap with previously reported lifespan and reproduction genes To examine possible support for the notion that the 137 probe sets have a strong association with lifespan, we compared our results to three studies that affected lifespan by different manipulations (Bauer et al. 2010; Doroszuk et al. 2010; Yamamoto et al. 2013). In total, 5549 probe sets were significantly affected in all three studies and from this list, 105 overlapped with our 137 found genes to be associated with lifespan. This $76.6 \%$ overlap is significantly larger than expected by chance (Chi-square, $\mathrm{p}<0.001$ ). Also, for the 443 probe sets that correlated to reproduction differences between our treatments we found a large and significant overlap of 319 probe sets with the other studies (Chi-square, $\mathrm{p}<0.001$ ). All other probe sets lists had a much lower proportionate overlap with those of the meta-analysis. For example, the overlap between the meta-analysis list and the probe sets differentially expressed between the two controls was $40.1 \%$, and between CL versus yoyo 53.2\%, and for those associated with the egg laying 33.9\% (all non-significant; Chisquare, $\mathrm{p}>0.1$, Figure $3 \mathrm{a}$ ).

\section{Co-expression network analysis}

Finally, we verified the association of the probe set lists with the measured phenotypes by performing a co-expression analysis. We tested significant co-expression between probe sets of all 32 samples and assembled four networks from this analysis, shown in Figure 3b. In total, 2,199 of all 18,952 probe sets contributed to the network analysis that consists of four modules that vary in size: 1375, 342, 49, and 22 probe sets respectively (Figure 3b). The mean number of connections in these modules was $38.0,15.3,4.5$, and 3.5 respectively.

We performed a PCA on the probe sets per module and quantified the variation of PC1 in response to nutrition and yoyo status (control or yoyo sample). For module 1 we found no clear association 
with the treatments, but for module 2 the variation between a low and high diet was larger between controls than in yoyo samples (Figure 3c), a pattern that resembles the reproduction response of these treatments (Figure 1a). For module 3, CH and CL samples showed a large difference, i.e. high PC values for $\mathrm{CH}$ and low for $\mathrm{CL}$ flies, but interestingly, the yoyo samples resembled the high control in the values of PC1, thereby resembling the treatment effects on survival (Figure 1b). Lastly, module 4 showed variation between the two diets, for both controls and yoyo flies, and was therefore associated with low or high food and similar to module 2 , consistent with the fluctuations in reproduction (Figure 3c).

When we related the loading values of the first principal component of the probe sets of each module to those of all probe sets, module 1 had a significant positive correlation with PC1, and negative correlation with PC2 (Figure 3d). Although both were significant (Pearson correlation, $\mathrm{p}<0.05$ ), the correlation coefficient between PC1 of module 2 to PC1 of all probe sets showed a more significant positive correlation (Figure 3d). Furthermore, PC1 of module 3 correlated significantly to PC2 of all probe sets, and module 4 showed again a correlation to PC1 (Figure 3d). To examine further possible support for the association of probe sets to our measured phenotypes reported in Figure 3a (yellow and red area), we tested the overlap between the probe sets of each module and each of the Venn-diagram categories shown in Figure 3a. This showed for the first module an overlap of $12 \%$ and $11 \%$ with the probe sets of the subgroup of 'reproduction genes' and 'core reproduction genes' (respectively shown in red and yellow in Figure 3a and 3e). For module 2, however, a 77\% overlap was observed only with the 'core reproduction' probe sets (colored yellow, Figure 3a and 3e). Interestingly, the probe sets of module 3 overlapped most with the 'lifespan' core list from Figure 3, having an overlap of 51\% (Figure 3e).

Lastly, we identified 'hubs', the genes with most connections in each module. For module 1, we identified 14 genes with more than 218 connections. These genes all have regulatory functions in processes such as transcription, meiosis, and the cell cycle (e.g $C y c A$ ), and all have a highly ovaryspecific expression (Table 1). Similarly to the hubs of the first module, the hubs of module 2 were also regulatory genes, but for this module the genes are mainly expressed in nervous tissue (Table 1). For module 3 one hub was found, CG9380, a gene associated with peptidoglycan catabolism and expressed in the digestive system. Similar to module 1, the four hubs found in module 4 are all ovary specific genes, but effector rather than regulatory genes. 
a

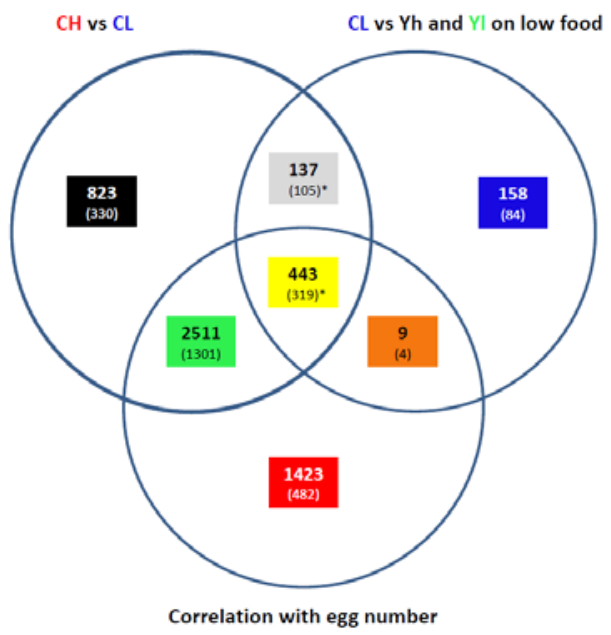

b
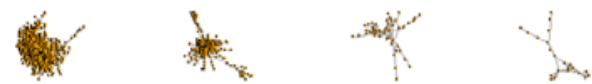

C
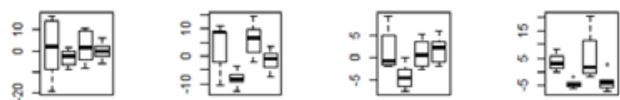

CHCLYHYL

CHCLYHY

CHCLYHYL

CHCLYHY

d
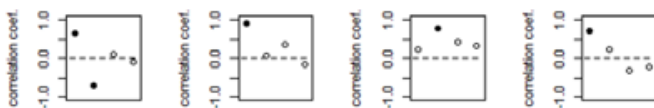

PC1 PC2 PC3 PC4

PC1 PC2 PC3 PC4

PC1 PC2 PC3 PC4

PC1 PC2 PC3 PC4

e
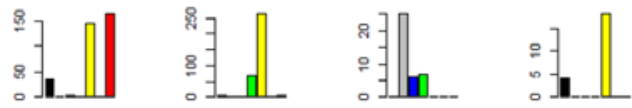

Figure 3. (a) Venn diagram of the overlap in probe sets significantly up- or downregulated between different treatments and those related to the number of eggs laid and. The upper left circle represents the 3914 genes differently expressed between CH and CL flies, the upper right circle the 747 genes differently expressed between CL and yoyo flies on low food, and the below circle the 4386 genes significantly correlated with the number of eggs. In the coloured boxes the number of overlapping probe sets between groups are given; e.g. the grey box represents the 137 probe sets that are differentially expressed between CH and CL and also between CL and yoyo flies on low food, but do not overlap the probe set list that correlates to reproduction. Between brackets the number of probe sets is indicated that were also differently expressed in our meta-analysis cohort, and asterisks $\left(^{*}\right)$ indicate a significant overlap with this cohort. (b) The 2D structure of each of the four assembled networks that were derived from a performed co-expression analysis on all samples. (c) For each treatment per network boxplots of PC1values are shown of control and yoyo treatments. (d) Correlations between PCA1-4 of all probeset and the PC1 of each network, filled dots represent a significant positive $(<0)$ or negative $(>0)$ correlation). (e) Number of overlapping probe sets between each (in figure a) assigned probe set list (by colours) and the probe sets assigned to each network. For each network, in table 1 the highest connected genes for each network are listed. 
Table 1. A description of highly connected genes (hubs) of the four co-expression networks. For each gene the function, the number of co-expressed genes, and predominant tissue of its expression is given.

\begin{tabular}{lllll}
\hline & Gene symbol & Function & Co-expression & Tissue \\
\hline CG8478 & Zinc finger & 290 & Ovary \\
& CG519 & Proteasome localization & 299 & Ovary \\
insv & Transcription corepressor & 277 & Ovary \\
Network1 & Zwilch & Cell cycle regulator & 247 & Ovary \\
& CG14965 & Nucleid acid binding & 221 & Ovary \\
& Chd3 & Helicase, nucleosome rem. & 228 & Ovary \\
Mus81 & DNA repair & 224 & Ovary \\
fizzy & Regulator of meiosis & 237 & Ovary \\
Torso & Tyrosine protein kinase & 233 & Ovary \\
& Mcm10 & Chromosome condensation & 232 & Ovary \\
mei-S332 & meiosis & 237 & Ovary \\
CycA & Cell cycle regulator & 234 & Ovary \\
& Irbp & Helicase, DNA binding & 235 & Ovary \\
& Hmr & Telomere maintenance & 222 & Ovary \\
& & & \\
Appl & Learning & 76 & Nervous tissue \\
Rab3 & Rab signal transduction & 82 & Nervous tissue \\
CG2269 & Unknown & 83 & Nervous tissue \\
RIC-3 & Chaperone mediated protein binding & 82 & Various \\
& & & \\
CG9380 & Peptidoglycan catabolic process & 15 & Digestive system \\
Network2 & & & Ovary \\
Muc4B & Eggshell assembly & 6 & Ovary \\
CG13114 & Chorion & 6 & Ovary \\
CG13737 & Unknown & 6 & Ovary \\
\hline CG4009 & Heme oxidase & 6 &
\end{tabular}

\section{DISCUSSION}

We set out to study the flies' life history and transcription responses to an alternating (i.e. yoyo) diet throughout life to test whether flies exhibit consistent variation in gene expression between a high and low diet, how this variation compares to differences between control flies of both diet conditions, and how the variation in gene expression can be linked to direct effects of diet and to reproduction and lifespan phenotypes.

\section{Large and rapid responses of yoyo flies: phenotypes and whole genome transcription}

In our previous study we showed that yoyo flies lived longer than flies on a control low (CL) diet and altered reproduction quickly to a changing nutritional condition (Van den Heuvel et al. 2014; (Figure 1). In the present study we showed that this extreme phenotypic plasticity of the flies was reflected in their whole genome transcription; within four days and irrespective of the direction of 
diet change, the whole genome transcription pattern changed from comparable to the one control to the other (Figure 2). To our knowledge, the only other studies measuring effects of diet fluctuation during life on the level of whole genome transcription tested the effects of a single diet switch (Gershman et al. 2007; Whitaker et al. 2014). The results of both studies are in concordance with our result that a diet switch yields an immediate and large transcriptomic change. Compared to our study many fewer genes $(n=144)$ were differentially expressed after a diet switch in the study where RNA was used from only heads and thoraces (Whitaker et al. 2014), but a more similar number of affected genes $(n=3,519)$ was found when, as in our study, RNA of whole flies was used (Gershman et al. 2007). Additionally, our results indicate that flies can respond to diet changes throughout life and that this is not affected by diet fluctuations that occurred in the past. This suggest a strong adaptive potential of fruit flies to cope with a dynamic environment through a rapid and radical change in its whole genome transcription pattern, in turn leading to a large change in the flies' life-history traits.

\section{Transcription patterns consistently relate to lifespan and reproduction phenotypes}

Although our observed phenotypes were replicated and consistent with our previous experiments (Van den Heuvel et al. 2014, Figure S3), these patterns were different from a typical dietary restriction (DR) phenotype where increased nutrition is generally associated with enhanced reproduction and decreased lifespan (Piper \& Partridge, 2007). However, it has been well documented that phenotypic responses to diet are easily affected by differences in diet composition, genotype, group size, and mating status (Chapman et al. 1994; Ackerman et al. 2001; Skorupa et al. 2008; Doroszuk et al. 2012; Piper et al. 2014; Zandveld et al. 2017). For example, in Queensland fruit flies' mating status also interacted with the response to diet composition (Fanson et al. 2013), and likewise, this interaction is also true for our fruit fly population (Figure S5), confirming that these factors play an essential role in the fly's life-history response.

The genes for which expression was affected by the conditions in this study showed a large overlap with the genes affected in the lifespan manipulations in our meta-analysis and with other studies (Farhadian et al. 2012; Pletcher et al. 2002). This suggests that any nutritional manipulation that shows a similar phenotypic contrast between treatments, i.e. lifespan extension and decreased reproduction, will also result in a similar transcription pattern. A possible consequence of this observation is, however, that not all genes upregulated with diet are the specific mediators of the lifespan response to diet, but could very well be mediating other processes that also differ between diets. Despite previous attempts to eliminate effects of reproduction to interfere with gene expression changes with lifespan extension (Grandison et al. 2009; Antosh et al. 2011), co-varying traits between lifespan-extending treatments may result in very consistent transcription patterns between the studies, but complicates our ability to causally link gene expression results to individual life-history traits. 
Relating variation in gene expression to reproduction, diet, and lifespan.

Here, we demonstrate that it is essential to measure multiple phenotypes to gain this type of mechanistic insight into a complex system. By measuring reproduction of our micro-array samples, we observed that the flies' transcription as captured in PC1 correlated with reproduction differences between the samples (Figure 2). The probe sets that showed no correlation with reproduction but a differential expression between the treatments that differ in lifespan are therefore more likely to be regulators of the lifespan response to diet.

The significant overlap of our "enriched lifespan probe set" with other micro-array studies on lifespan (Bauer et al. 2010; Doroszuk et al. 2012; Yamamoto et al. 2013), together with the observation that among this list of 137 genes hardly any are expressed in ovaries, strongly suggests that our enriched lifespan probe set is most likely functionally linked to lifespan. The annotated gene functions in this list vary considerably, from seemingly more downstream cellular processes, such as oxidation reduction (CG2604, Cyp313a1, CG14997, CG6279), transmembrane transport (vps2, Sec61gamma, Ugt86Dd), transcription (CG7255, nub), and endopeptidase activity/proteolysis (CG10992, multiple members of the Jon-family, CG30090), to more regulatory functions, such as ion binding (Orct, Cyp313a1, Yippee), transcription factor activity (Sox100B, CG15043), and hormone binding (burs, Npc2f, Eip75B, CG17189). This variety of different gene functions suggests that observed lifespan responses to diet are unlikely to be mediated through one linear pathway ( $c f$. Mair \& Dillin, 2007).

The rapid and clear reproduction response to diet allowed us to identify 433 genes that we call our "core reproduction gene set". Also in this gene set, we identified genes expressed in a variety of tissues and some are involved in downstream processes (e.g. GRHR, Lip3, Lsd-1, Hr96, CG34127), but many more genes show an expression predominantly in regulatory tissues such as head and fat body, and have often regulatory functions including hormone binding (dawdle, $N p c 2 b / 2 d$, takeout, CG31189, ImpL3) or transcription factor activity (Eip75B, Obp8a, Pvf1, Dll, abd-A). Interestingly, among our 'core reproduction genes' we identified takeout and dawdle, two genes that show a lifespan extension when overexpressed (Bauer et al. 2010; Bai et al. 2013). However, takeout deletion also changes feeding behavior, locomotion, circadian timing (Sarov-Blat et al. 2000; Meunier et al. 2007; Wong et al. 2009), and, interestingly, also courtship and fertility (Chamseddin et al. 2012). These multiple phenotypes associated with takeout or dawdle action (Bai et al. 2013), suggest these genes may have pleiotropic effects. Indeed, takeout operates through an interaction with juvenile hormone (Sarov-Blat et al. 2000, Chamseddin et al. 2012) and dawdle is connected to insulin activity (Bai et al. 2013), both examples of pathways that affect multiple life-history traits, including lifespan and reproduction (reviewed by: Leroi et al. 2005; Flatt \& Kawecki, 2007; Flatt, 2011).

In line with this, our 'core reproduction' list has a significant overlap with the meta-analysis transcription studies on lifespan (Figure 3a). Diet and genetic interventions that increase lifespan 
in Drosophila often also affect reproduction (Stearns, 1992; Zwaan et al,. 1994; Flatt, 2011; Emran et al. 2014; May et al. 2015; Zandveld et al. 2017). It is possible, therefore, that transcription studies on interventions affecting lifespan frequently show reproduction-related processes to be enriched in gene ontology analyses (Pletcher et al. 2002; Bauer et al. 2010; Doroszuk et al. 2010). We argue that when only measuring one trait, the genes that mediate other unmeasured co-varying traits will likely be associated with this trait, resulting in many false positives. We therefore highlight the importance of measuring multiple phenotypes for an accurate interpretation of the transcription patterns, showing in this case that it is possible to decouple genes involved in the diet response of lifespan and reproduction.

\section{Co-expression analysis}

To further explore the role of the identified genes in nutritional responses, we performed a coexpression analysis which resulted in four co-expression gene networks. Patterns of co-expression suggest the existence of functional gene networks underpinning the biology of certain traits (Mackay, 2004), for example lifespan and reproduction responses to diet. Indeed, our four networks showed a remarkably large overlap with our core sets of both lifespan (network 3 ) and reproduction genes (network 1, 2, and 4; Figure 3e).

From the three networks associated with reproduction, the first network showed a large overlap with our 'core reproduction gene set', but also with other probe sets that correlated to reproduction (yellow and red box, Figure 3). The most connected genes (hubs) were all expressed in ovaries and predominantly indicate functions linked to 'cell division' (Table 1) and have been reported to alter reproduction. For example, $\mathrm{Hmr}$ is required for ovariole formation in D. melanogaster (Aruna et al. 2009) and fizzy is known to inhibit mitosis, and affects another hub from this network $C y c A$ (Sigrist \& Lehner, 1997). This suggests that network 1 is involved in mediating reproduction downstream, mainly organized at the cellular level. The two other networks show overlap with our 'core reproduction' gene list on different functional levels. Namely, hubs of network 2 were mainly expressed in nervous tissue, and may therefore mediate the observed reproduction responses in a more central regulatory way, for instance $A p p l$ is a gene expressed in neuronal tissue, and is related to olfactory memory (Bourdet et al. 2015). Network 4 showed, similar to the first network, hubs that were primarily expressed in ovaries, but, different to network, are mainly involved in chorion assembly (Table 1), suggesting that this network acts on another level than cellular regulation represented by network 1 .

Network 3 appears to represent the lifespan effects of diet change and has one hub, CG9380. The predominant expression of this gene in the gut is in line with a regulatory function in the response to diet, and its role in peptidoglycan catabolism (flybase) points towards an immunity response also previously associated with a lifespan response upon diet change (Pletcher et al. 2002). An 
ortholog of this gene is also found in humans, where it is involved in the recognition of peptidoglycan, a component of bacterial cell walls (Schleifer \& Kandler 1972).

The co-expression analysis thus supported the gene lists associated with the reproduction and lifespan diet responses in this study (Figure 3a-e). It also captured some of the complex genetic architecture of life-history responses. Namely, the differences between the networks associated to reproduction indicate that this response is mediated through different regulatory levels, from central to more cellular. Our results further indicate many fewer transcripts to be involved in mediating the lifespan response, the largest transcriptomic changes observed being mainly a reflection of the fly's reproduction response.

\section{CONCLUSION}

The large and fast transcriptomic changes observed in this study showed that flies respond very plastically to diet changes already in the early steps along the genotype-phenotype map. This indicates that flies are able to shift their gene expression to fine-tune their suite of life history traits to the immediate nutritional environment on a very short time scale, which is likely to be adaptive in natural populations. As a consequence, in order to dissect trait-specific groups of effector genes, it is crucial to measure multiple life history traits for any laboratory lifespan or reproduction intervention. Indeed, lifespan differs among treatments, however, many differentially expressed genes are not necessarily indicative for lifespan changes but rather for other co-varying traits, such as a differential reproduction pattern. 


\section{Acknowledgements}

We thank Scott Pletcher for sharing his micro-array data, Agnieszka Doroszuk and Vicencio Oostra for advice on statistical analysis and Kees Koops for help in fly rearing, medium cooking, and assistance with experiments. This study was supported by the Network of Excellence LifeSpan (FP6 036894), the European Union's Seventh Framework Programme (FP7/2007-2011) under grant agreement number 259679, and and the European Research Council.

\section{SUPPLEMENTAL MATERIAL}

\section{Figure S1}

Experimental set up

\section{Figure S2}

Egg production micro-array cohort

\section{Figure S3}

Principle component analysis (PCA) for four independent trials

\section{Figure S4}

Ven-diagram all diet contrasts

\section{Figure S5}

Effects of mating status and diet regimes on reproduction

\section{Table S1}

Statistical analyses

\section{Table S2}

Lifespan gene set 


\section{Figure S1}

\section{Experimental set up}

\section{experimental setup}

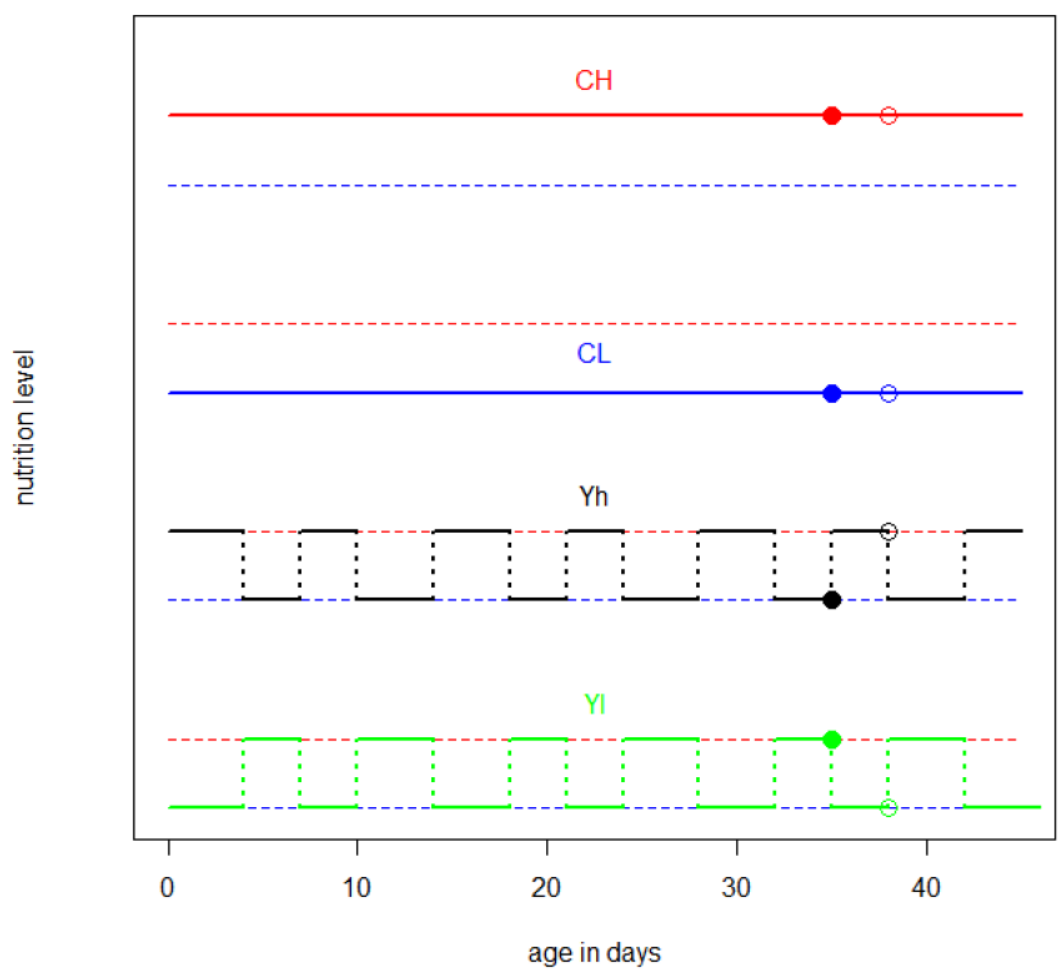

Figure S1. Schematic overview of the experimental setup. The four food treatments vary in when flies were on high (red lines) or low (blue lines) nutritious food. The control high $(\mathrm{CH})$ and control low $(\mathrm{CL})$ treatment flies were respectively on high and low food throughout the experiment. The yoyo high treatment flies $(\mathrm{YH})$ were on high food for four days and then altered food condition every 3 or 4 days, yoyo low treatment flies (YL) followed a reversed pattern to $\mathrm{YH}$, starting on low food. For gene expression analysis fly samples were collected at the ages 34 (filled dots) and 38 days (empty dots). 


\section{Figure S2}

\section{Egg production micro-array cohort}

egg production microarray cohort

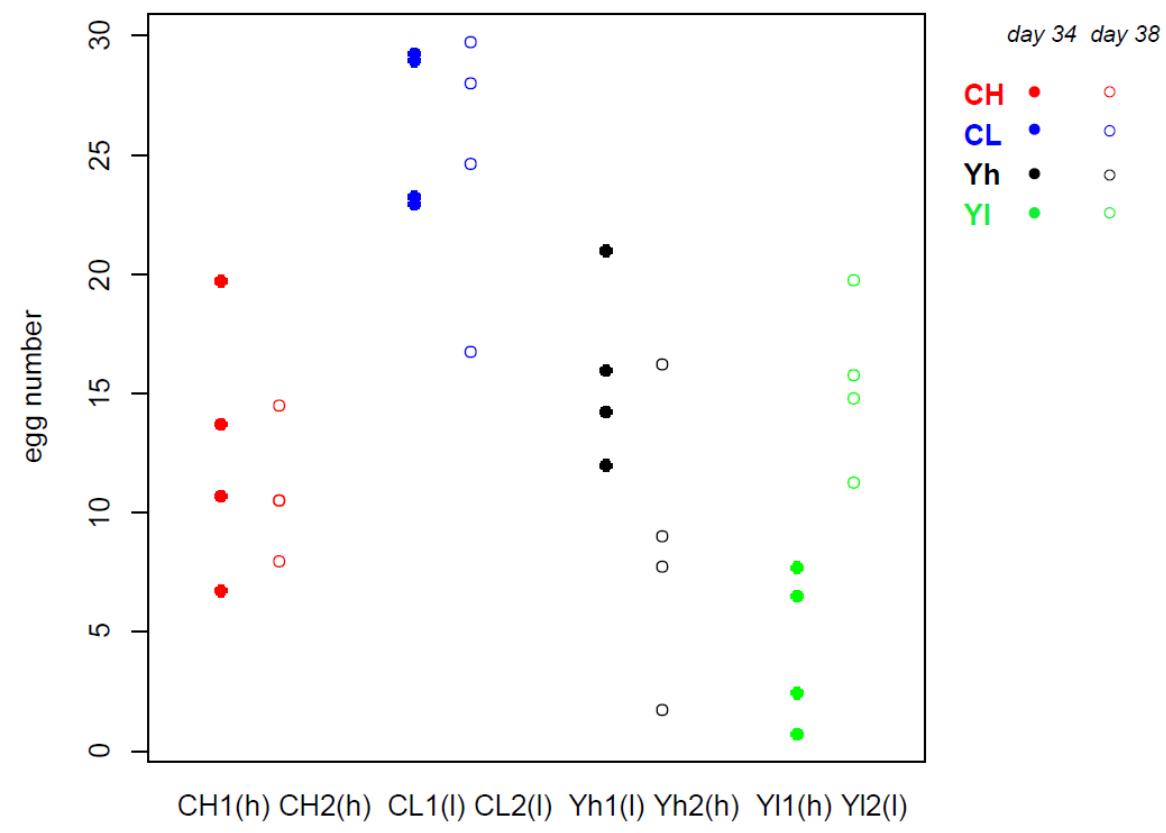

Figure S2. Number of eggs produced by flies in the microarray cohort before sampling. Treatments are indicated at the X-axis; the time point of sampling is indicated by the number ( 1 or 2$)$, and between brackets the food type the flies were on at the time of sampling. 


\section{Figure S3}

Principle component analysis (PCA) for four independent trials
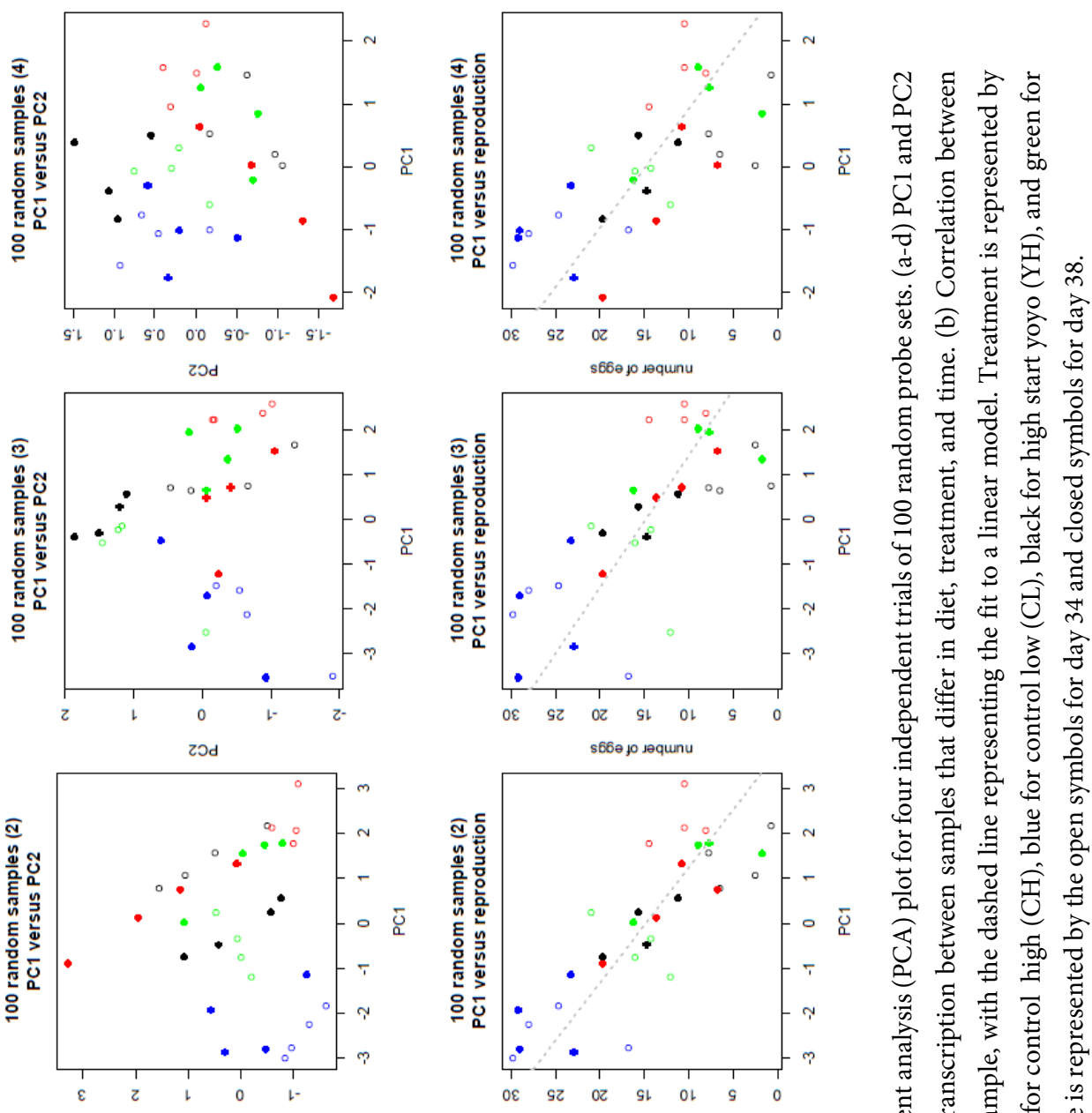

है च चं की

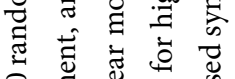

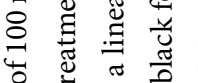

¿

祃 苛过

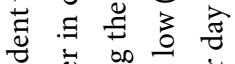

范

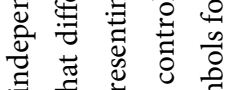
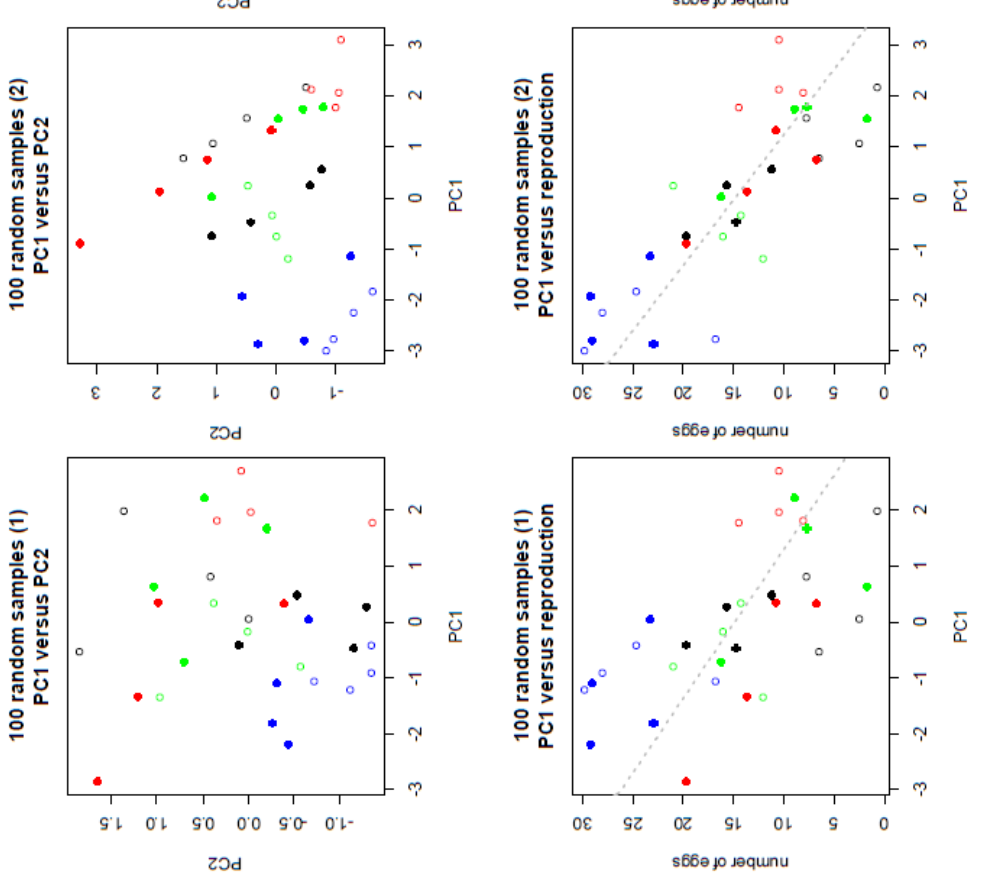

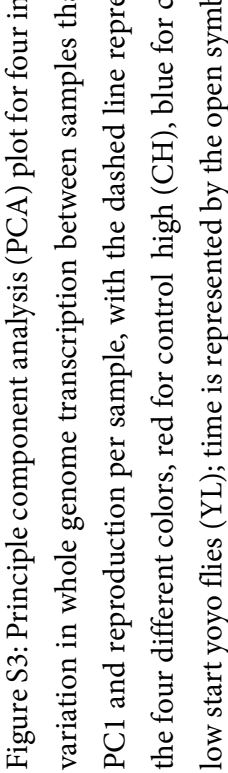




\section{Figure S4}

\section{Ven-diagram all diet contrasts}

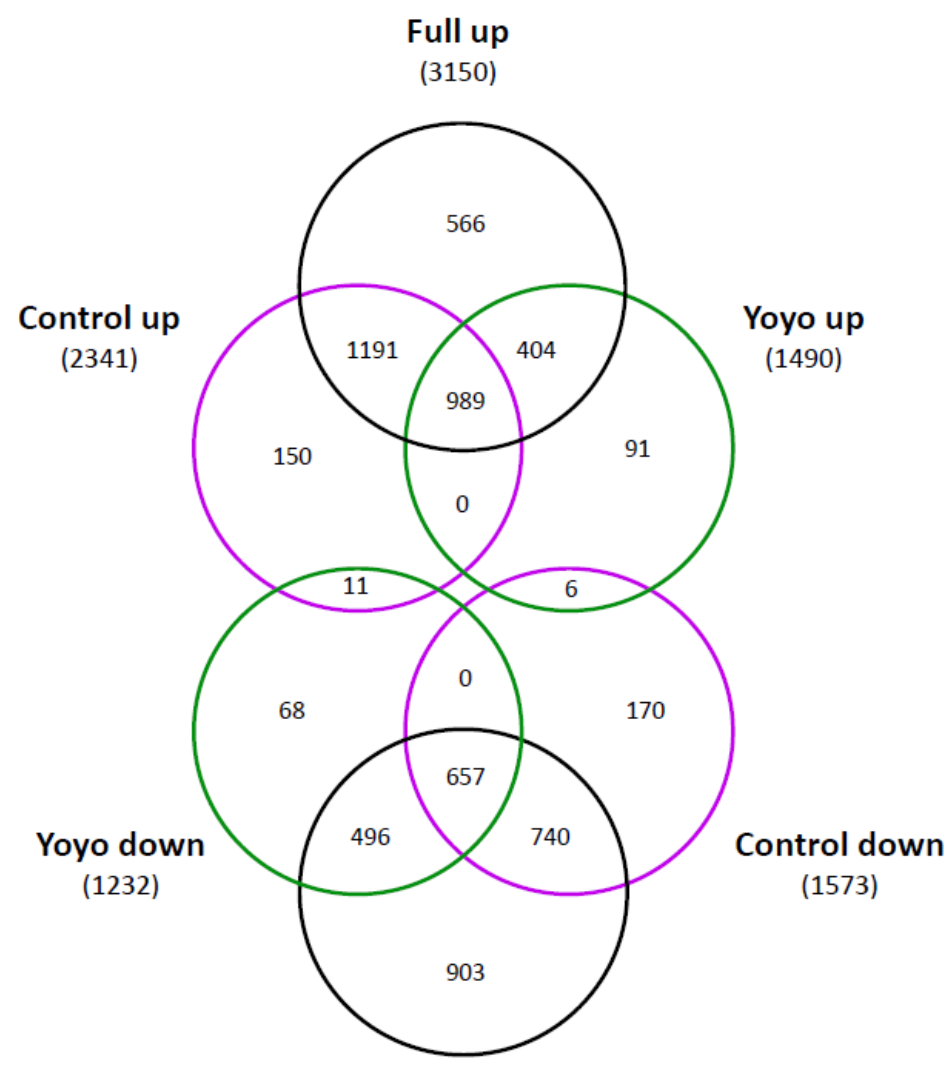

\section{Full down}

(2796)

Figure S4: Venn diagram of significant up- and downregulated probe sets with diet for all samples together (full up/down), and separately for the control and yoyo treatments (control up/down and yoyo up/down). 


\section{Figure S5}

\section{Effects of mating status and diet regimes on reproduction}
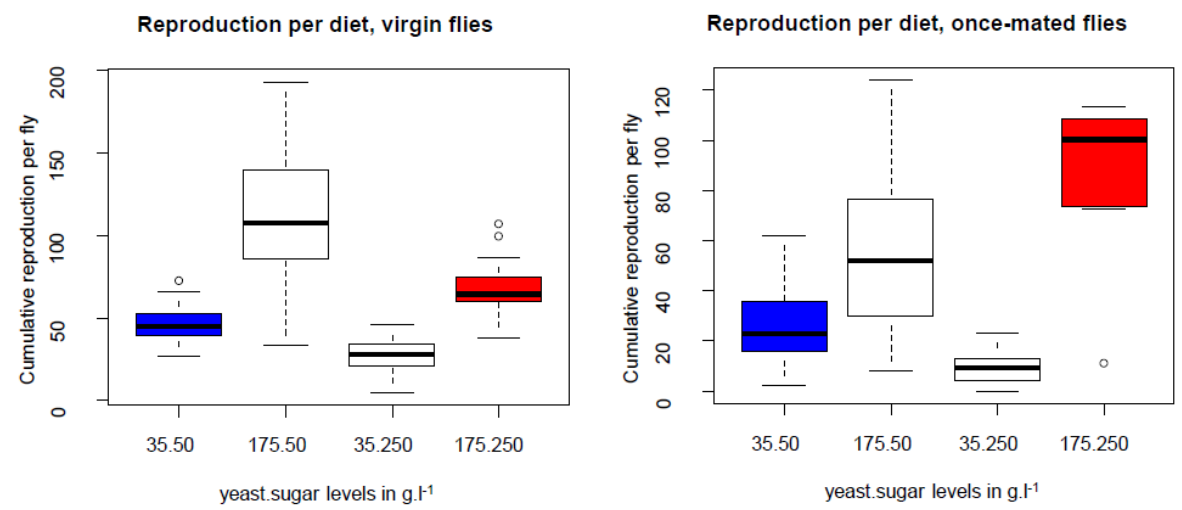

Figure S5. Average fecundity per day of (a) virgin and (b) once-mated flies per diet treatment in another cohort of fruit flies. Diet treatments are indicated by the level of yeast and sugar (in g..$^{-1}$ ) respectively, blue and red boxplots correspond respectively to the low and high food treatment of our current study. Although virgin flies on high food (175.250 YS) reproduce more than on low (35.50 YS), the reproduction response depends both on the flies' mating status and the diet composition. 


\section{Table S1}

\section{Statistical analyses}

Table S1.1: Summary of the principle component analysis of the whole genome expression data. For each principle component the (cumulative) variation explained $+/$ - standard deviation.

\begin{tabular}{llll}
\hline $\begin{array}{l}\text { Principle } \\
\text { component }\end{array}$ & $\begin{array}{l}\text { Standard } \\
\text { deviation }\end{array}$ & $\begin{array}{l}\text { Proportion } \\
\text { of Variance }\end{array}$ & $\begin{array}{l}\text { Cumulative } \\
\text { Proportion }\end{array}$ \\
\hline & & & \\
PC1 & 19.8782 & 0.3077 & 0.3077 \\
PC2 & 15.0799 & 0.1771 & 0.4848 \\
PC3 & 10.3050 & 0.0827 & 0.5675 \\
PC4 & 8.6497 & 0.0583 & 0.6257 \\
PC5 & 6.5193 & 0.0331 & 0.6589 \\
PC6 & 6.2628 & 0.0305 & 0.6894 \\
PC7 & 5.7511 & 0.0258 & 0.7151 \\
PC8 & 5.2507 & 0.0215 & 0.7366 \\
PC9 & 5.1013 & 0.0203 & 0.7569 \\
PC10 & 4.7674 & 0.0177 & 0.7746 \\
& & & \\
\hline
\end{tabular}


Table S1.2: Number of significant probe sets up- and down regulated in specific contrasts (MAANOVA, $\mathrm{p}<0.05)$. Four types of contrasts are tested: high against low food samples, first against second time point, control against yoyo samples and $\mathrm{Yh}$ against $\mathrm{Yl}$ lines using different sets of samples as indicated in the left column, within brackets the number of samples used for this analysis.

\begin{tabular}{|c|c|c|c|}
\hline \multicolumn{4}{|l|}{ Food effects: high food versus low food } \\
\hline $\begin{array}{l}\text { Within } \\
\text { All samples (32) }\end{array}$ & $\begin{array}{l}\text { Genes significant } \\
5946\end{array}$ & $\begin{array}{l}\text { Up on high } \\
3150\end{array}$ & $\begin{array}{l}\text { Down on high } \\
2796\end{array}$ \\
\hline Control samples (16) & 3914 & 2341 & 1573 \\
\hline Yoyo samples (16) & 2722 & 1490 & 1232 \\
\hline Yoyo low start (8) & 2004 & 1155 & 849 \\
\hline Yoyo high start (8) & 495 & 244 & 251 \\
\hline \multicolumn{4}{|c|}{ Time effects: first time point versus second time point } \\
\hline $\begin{array}{l}\text { Within } \\
\text { All samples (32) }\end{array}$ & $\begin{array}{l}\text { Genes significant } \\
4424\end{array}$ & $\begin{array}{l}\text { Up in first } \\
2848\end{array}$ & $\begin{array}{l}\text { Down in first } \\
1576\end{array}$ \\
\hline Control samples (16) & 5251 & 3425 & 1826 \\
\hline Yoyo samples (16) & 0 & 0 & 0 \\
\hline High food (16) & 4561 & 2919 & 1642 \\
\hline Low food (16) & 0 & 0 & 0 \\
\hline \multicolumn{4}{|l|}{ Effects of yoyo: control lines versus yoyo lines } \\
\hline $\begin{array}{l}\text { Within } \\
\text { All samples (32) }\end{array}$ & $\begin{array}{l}\text { Genes significant } \\
74\end{array}$ & ${ }_{26}^{U_{p} \text { for yoyo }}$ & $\begin{array}{l}\text { Down for yoyo } \\
48\end{array}$ \\
\hline yoyo on low food vs control on low food (16) & 747 & 483 & 264 \\
\hline yoyo on high food vs control on high food & 0 & 0 & 0 \\
\hline \multicolumn{4}{|l|}{ Effects of start food type: Yh versus Yl lines } \\
\hline $\begin{array}{l}\text { Within } \\
\text { Yh vs Yl (16) }\end{array}$ & $\begin{array}{l}\text { Genes significant } \\
0\end{array}$ & $\begin{array}{l}\text { Up for yoyo } \\
\text { low } \\
0\end{array}$ & $\begin{array}{l}\text { Down for yoyo } \\
\text { low } \\
0\end{array}$ \\
\hline Yl on low food vs Yh on low food (8) & 0 & 0 & 0 \\
\hline Yl on high food vs Yh on high food (8) & 0 & 0 & 0 \\
\hline
\end{tabular}




\section{Table S2}

\section{Lifespan gene set}

Table S2: extracted gene list for lifespan effects between treatments. For each gene their function (FlyBase Consortium, 2016) and tissue specific expression pattern (flyatlas: Chintapalli et al. 2007) are listed. ND: not determined function; A: adult expression; L larval expression; AL both adult and larval expression.

\begin{tabular}{|c|c|c|}
\hline gene id & gene ontology (flybase) & tissue (fly atlas) \\
\hline Eip75B & DNA binding, heme binding & ubiquitous \\
\hline Gs2 & $\begin{array}{l}\text { glutamate catabolic process, neurotransmitter receptor metabolic process, } \\
\text { synapse assembly, glutamine biosynthetic process }\end{array}$ & ubiquitous \\
\hline Jon $44 E$ & endopeptidase & AL: midgut \\
\hline$i f c$ & breakdown fatty acids & ubiquitous \\
\hline$L v p D$ & carbohydrate metabolic process & A salivary gland; AL gut \\
\hline Jon99Ci & endopeptidase & $\begin{array}{l}\text { A: salivary gland, AL: } \\
\text { midgut }\end{array}$ \\
\hline$L y s X$ & antimicrobial properties & AL: midgut hindgut \\
\hline Pabp2 & polyadenylation, poly A shortening, neurogenesis & ubiquitous \\
\hline CG15865 & ND & AL gut \\
\hline $\begin{array}{l}\text { Lectin- } \\
\text { galC1 }\end{array}$ & $\begin{array}{l}\text { galactose binding, bacterial aggutination, ca+ dependent cell-cell } \\
\text { adhesion }\end{array}$ & $\begin{array}{l}\text { A heart, fat body; AL } \\
\text { carcass }\end{array}$ \\
\hline Orct & cation transport & $\begin{array}{l}\text { Ubiquitous excl A: } \\
\text { tubule, ovary }\end{array}$ \\
\hline Jon $25 B i$ & endopeptidase (inferred from sequence model) & AL: midgut \\
\hline Sox $100 B$ & male gonad dev, DNA binding, TF binding & A: gut, testis; L: gut \\
\hline $\operatorname{Tsp} 2 A$ & $\begin{array}{l}\text { smooth septate junction assembly, establishment of endothelial intestinal } \\
\text { barrier }\end{array}$ & $\begin{array}{l}\text { AL almost ubiquitous, } \\
\text { high in gut }\end{array}$ \\
\hline CG5885 & SRP-dependent cotranslational protein targeting to membrane & ubiquitous \\
\hline Yippee & metal ion binding, zinc ion binding & AL: gut \\
\hline$U g t 37 b 1$ & transferase, metabolism & A head, AL gut \\
\hline CG14777 & ND & ubiquitous, low in testes \\
\hline CG18480 & sensory of pain & A tubule, gut, ovary; L gut \\
\hline$C G 15772$ & structural molecule activity & $\begin{array}{l}\text { A Thorac gangl, brain, AL } \\
\text { fat body }\end{array}$ \\
\hline CG6041 & serine type endopeptidase & $\begin{array}{l}\text { A Thorac gangl, brain; AL } \\
\text { gut }\end{array}$ \\
\hline CG3106 & transferase acyl groups other than amino-acyl groups & $\begin{array}{l}\text { A Thoracicoabdominal } \\
\text { ganglion, brain, L: CNS }\end{array}$ \\
\hline CG15211 & marvel domain (regulation membrane structure) & $\begin{array}{l}\text { AL: ubiquitous, not in } \\
\text { ovary }\end{array}$ \\
\hline CG15739 & phosphatase activity & $\begin{array}{l}\text { AL: ubiquitous, mainly } \\
\text { midgut, not salivary gland } \\
\text { and Thorac gangl }\end{array}$ \\
\hline CG10992 & cysteine-type endopeptidase activty, endopeptidase & AL: ubiquitous \\
\hline CG15043 & activates transcription factor with TFIIA & AL: midgut \\
\hline
\end{tabular}




\begin{tabular}{|c|c|c|}
\hline CG7992 & ND & AL: Tubule, midgut \\
\hline CG14219 & transferase acyl groups other than amino-acyl groups & AL: midgut \\
\hline $\begin{array}{l}\text { Sec61gam } \\
m a\end{array}$ & protein transmembrane transporter activity & A: ubiquitous \\
\hline CG17264 & ND & ubiquitous \\
\hline CG3285 & transmembrane transport, integral component of membrane & $\begin{array}{l}\text { A: hindgut, crop, L S2 } \\
\text { cells }\end{array}$ \\
\hline CG3921 & $\begin{array}{l}\text { cadherence junction, septate junction, tricellular tight junction, open } \\
\text { tracheal system, response to endoplasm ret stress, establishment intestinal } \\
\text { barrier }\end{array}$ & $\begin{array}{l}\text { A Thora. Ganglion, crop, } \\
\text { midgut; L CNS, tubule, } \\
\text { hindgut, trachea }\end{array}$ \\
\hline CG15422 & ND & AL: gut \\
\hline Jon $25 B i i$ & neurogenesis, endopeptidase activity & AL: midgut \\
\hline obst-E & chitin binding & $\begin{array}{l}\text { AL carcass, hindgut, crop, } \\
\text { head; L trachea }\end{array}$ \\
\hline CG9468 & alpha-mannosidase activity, carbohydrate binding, zinc ion binding & AL: midgut \\
\hline Tsp29Fa & integral component of membrane & AL gut, A salivary gland \\
\hline CG13102 & ND & AL gut tubule \\
\hline CG17855 & ND & AL: gut, tubule \\
\hline CG14935 & catalytic activity, carbohydrate metabolic process & $\begin{array}{l}\text { L: low; A: Ubiquitous, } \\
\text { highest in fat body }\end{array}$ \\
\hline CG13082 & ND & A low: head; L: hindgut \\
\hline CG9319 & alpha-methulacyl-CoA racemase activity, fatty acid alpha-oxidation & AL: ubiquitous \\
\hline CG13160 & ND & A: hindgut; L: gut \\
\hline CG13155 & ND & $\begin{array}{l}\text { A: heart ,fat body, carcass } \\
\text {; L : - }\end{array}$ \\
\hline CG3884 & ND & $\begin{array}{l}\text { A: heart; L: S2 cells; AL: } \\
\text { gut }\end{array}$ \\
\hline CG13321 & neurogenesis & $\begin{array}{l}\text { A: ovary testis (both low); } \\
\text { AL tubule, gut, S2 cells }\end{array}$ \\
\hline CG13323 & ND & $\begin{array}{l}\text { AL: gut, tubule; L: fat } \\
\text { body }\end{array}$ \\
\hline CG18635 & integral component of membrane & AL: gut, tubule \\
\hline CG10910 & ND & AL: gut; L: tubule \\
\hline CG10912 & cold acclimation & AL: gut; L: tubule \\
\hline CG4363 & ND & AL: midgut \\
\hline CG4377 & ND & A: head; AL: midgut \\
\hline CG13488 & ND & $\begin{array}{l}\text { A: heart AL: midgut L: } \\
\text { tubule, hindgut, fat body }\end{array}$ \\
\hline CG6018 & detection of temperature stimulus involved in sensory perception of pain. & $\begin{array}{l}\text { A: crop, gut, tubule, male } \\
\text { accessory glands; L: CNS, } \\
\text { gut, tubule, carcass, S2 } \\
\text { cells }\end{array}$ \\
\hline CG13511 & ND & $\begin{array}{l}\text { A: midgut, tubule, testis; } \\
\text { L: gut, tubule }\end{array}$ \\
\hline CG9826 & transmembrane transport, integral component of membrane & $\begin{array}{l}\text { A: midgut, virgin } \\
\text { spermatheca, mated }\end{array}$ \\
\hline
\end{tabular}




\begin{tabular}{|c|c|c|}
\hline & & $\begin{array}{l}\text { spermatheca; L: salivary } \\
\text { gland, midgut }\end{array}$ \\
\hline CG3860 & ND & ubiquitous \\
\hline CG4781 & ND & AL: gut, tubule \\
\hline CG9380 & $\begin{array}{l}\mathrm{N} \text {-acetylmuramoyl-L-alanine amidase activity, peptidoglycan catabolic } \\
\text { process }\end{array}$ & $\begin{array}{l}\text { AL: gut, tubule; A: crop; } \\
\text { L: fat body }\end{array}$ \\
\hline CG14997 & oxidation reduction process & ubiquitous \\
\hline CG15829 & fatty-acyl-CoA binding & AL: gut \\
\hline CG6279 & oxidation reduction process & $\begin{array}{l}\text { A: heart, fat body, testis } \\
\text { (high), virgin and mated } \\
\text { spermatheca; L: fat body }\end{array}$ \\
\hline CG10657 & lateral inhibition, (neuronal) intrcellular, transporter activity & $\begin{array}{l}\text { A: head, crop, tubule, } \\
\text { hindgut, virgin and } \\
\text { mated spermatheca; L: } \\
\text { CNS, gut, trachea, } \\
\text { carcass; S2 cells }\end{array}$ \\
\hline CG10943 & ND & AL: gut \\
\hline CG13482 & ND & AL: gut, tubule; A: heart \\
\hline CG7255 & mRNA splicing, amino acid transmembrane transport & $\begin{array}{l}\text { A: salivary gland, gut, } \\
\text { tubule, testis, male acc. } \\
\text { glands, mated } \\
\text { spermatheca; L gut, } \\
\text { tubule, fat body, carcass; } \\
\text { S2 cells }\end{array}$ \\
\hline CG9701 & hydrolyze activity O-glycosyl, carbohydrate metabolism & $\begin{array}{l}\text { A: head, eye, crop, gut, } \\
\text { heart, fat body, testis, } \\
\text { carcass, spermatheca; L: } \\
\text { salivary gland, gut, } \\
\text { tubule, trachea, carcass }\end{array}$ \\
\hline CG5506 & ND & $\begin{array}{l}\text { A: head, sal gland, gut } \\
\text { (high), fat body, } \\
\text { spermatheca; L: gut } \\
\text { (high), CNS, tubule }\end{array}$ \\
\hline CG16775 & sensory percention of pain & AL: midgut; A: testis \\
\hline CG7298 & chitin binding, extracellular region & $\begin{array}{l}\text { AL: gut (high), testis; L: } \\
\text { gut(high), tubule }\end{array}$ \\
\hline CG2604 & $\begin{array}{l}\text { oxidation reduction process, chaeta development, wing disc development, } \\
\text { lipid particle }\end{array}$ & ubiquitous \\
\hline Сур $313 a 1$ & oxidation reduction process, heme binding, iron ion binding & $\begin{array}{l}\text { A: brain, head (high), eye } \\
\text { (high), heart, carcass; L: - }\end{array}$ \\
\hline CG14872 & ND & $\begin{array}{l}\text { ubiquitous; A: excl sal } \\
\text { gland, tubule, ovary, male } \\
\text { acc gland; L: excl CNS, sal } \\
\text { gland, tubule, trachea }\end{array}$ \\
\hline CG8925 & transmembrane transport, integral component of membrane & $\begin{array}{l}\text { A: head, eye, sal gland, } \\
\text { gut, male acc gland; L: sal } \\
\text { gland, midgut, trachea }\end{array}$ \\
\hline CG17752 & transmembrane transport, integral component of membrane & AL: tubule \\
\hline
\end{tabular}




\begin{tabular}{|c|c|c|}
\hline CG10824 & aspartic-type endopeptidase & $\begin{array}{l}\text { A: head, eye, hindgut, } \\
\text { heart, fat body, testis, } \\
\text { spermatheca; L: fat body }\end{array}$ \\
\hline burs & neuropeptide receptor binding, hormone activity & AL: gut; A: tubule; L: CNS \\
\hline CG5388 & ND & A: testis; L fat body (low) \\
\hline CG4723 & $\begin{array}{l}\text { proteolysis, metalloendopeptidase activity, cleavage neuropeptides (Isaac } \\
\text { et al. 2000) }\end{array}$ & $\begin{array}{l}\text { A: head, eye, heart, fat } \\
\text { body, spermatheca, } \\
\text { carcass; L: fat body }\end{array}$ \\
\hline CG10182 & transferase activity, transferring acyl groups other than amino-acyl groups & AL: midgut; L: hindgut \\
\hline$N p c 2 f$ & steriol binding, steriol transport & $\begin{array}{l}\text { A: head, midgut (high), } \\
\text { heart, fat body, testis, } \\
\text { spermatheca, carcass; L: } \\
\text { CNS, midgut (high); } \\
\text { hindgut, fat body; S2 cells }\end{array}$ \\
\hline CG10514 & ND & $\begin{array}{l}\text { A: brain, head, eye. Thor } \\
\text { gagnlion, tubule (high), } \\
\text { hindgut, testis, } \\
\text { spermatheca, carcass, } \\
\text { tubule (high), hindgut }\end{array}$ \\
\hline$v p s 2$ & $\begin{array}{l}\text { endosomal transport, regulation of notch signalling pathway, colocalizes } \\
\text { with early endosome/multivesicular body, ESCRTIII complex }\end{array}$ & ubiquitous \\
\hline CG17189 & $\begin{array}{l}\text { sensory perception of pain, extracellular space, family haemolymph JH } \\
\text { binding }\end{array}$ & A: head, carcass; L: - \\
\hline CG12428 & $\begin{array}{l}\text { carnitine O-octanoyltransferase activity, fatty acid beta-oxidation, } \\
\text { peroxisome }\end{array}$ & ubiquitous \\
\hline CG9988 & catalytic activity, metabolic process & AL: midgut \\
\hline CG15553 & transmembrane transport, integral component of membrane & $\begin{array}{l}\text { A: head, eye, heart, fat } \\
\text { body, spermatheca, } \\
\text { carcass; L: fat body }\end{array}$ \\
\hline CG11318 & $\begin{array}{l}\text { G-protein coupled receptor signaling pathway, cell surface receptor } \\
\text { signaling pathway, integral component of membrane }\end{array}$ & $\begin{array}{l}\text { AL: midgut; A: male acc } \\
\text { glands }\end{array}$ \\
\hline CG11550 & ND & $\begin{array}{l}\text { A: head, eye, gut (high } \\
\text { hindgut), male acc glands, } \\
\text { carcass; L: trachea, } \\
\text { carcass }\end{array}$ \\
\hline Ugt86Dd & $\begin{array}{l}\text { glucuronosyltransferase activity, transferase activity, transferring hexosyl } \\
\text { groups, metabolic process }\end{array}$ & $\begin{array}{l}\text { A: ubiquitous except } \\
\text { ovary and male acc } \\
\text { glands; L: gut, tubule, fat } \\
\text { body }\end{array}$ \\
\hline CG32795 & $\begin{array}{l}\text { defence response gram positve bacteria, positive regulation innate } \\
\text { immune response, intergal component of memrane }\end{array}$ & ubiquitous \\
\hline CG15210 & ND & $\begin{array}{l}\text { A: brain, head, eye, sal } \\
\text { gland, crop, gut, mated } \\
\text { spermatheca; L: } \\
\text { ubiquitous except carcass }\end{array}$ \\
\hline yellow-e & ND & $\begin{array}{l}\text { A: head, eye, hundgut, } \\
\text { adult carcass (high); L: }\end{array}$ \\
\hline
\end{tabular}




\begin{tabular}{|c|c|c|}
\hline & & $\begin{array}{l}\text { hindgut, trachea, carcass } \\
\text { (high) }\end{array}$ \\
\hline CG18869 & transferase activity, transferring hexosyl groups, metabolic process & $\begin{array}{l}\text { A: gut, testis; L: gut, fat } \\
\text { body }\end{array}$ \\
\hline CG10650 & ND & $\begin{array}{l}\text { A: Thorac gangl, sal } \\
\text { gland, gut, tubule, testis, } \\
\text { male acc glands, } \\
\text { spermatheca; L: sal gland, } \\
\text { gut, tubule, trachea }\end{array}$ \\
\hline Cpr49Ab & $\begin{array}{l}\text { structural constituent of chitin-based larval cuticle, structural constituent } \\
\text { of cuticle, chitin based cuticle development }\end{array}$ & $\begin{array}{l}\text { A: fat body, carcass } \\
\text { (high); L: - }\end{array}$ \\
\hline CG30090 & serine-type endopeptidase activity, proteolysis & $\begin{array}{l}\text { A: head, eye, midgut, } \\
\text { heart, fat body, testis, } \\
\text { spermatheca; L : carcass; } \\
\text { S2 cells }\end{array}$ \\
\hline CG30197 & peptidase inhibitor activity, extracellular region & $\begin{array}{l}\text { ubiquitous, except larval } \\
\text { carcass and S2 cells }\end{array}$ \\
\hline CG31004 & smooth septate junction assembly, cell-matrix adhesion & $\begin{array}{l}\text { A: gut, tubule, } \\
\text { spermatheca; L: gut, } \\
\text { tubule }\end{array}$ \\
\hline CG31091 & hydrolase activity, acting on ester bonds, lipid metabolic process & AL: midgut \\
\hline Mur29B & extracellular matrix structural constituent & AL: gut, tubule \\
\hline CG32054 & transmembrane transporter activity, integral component of membrane & AL: gut; L: tubule \\
\hline CG32107 & ND & AL: gut, tubule \\
\hline CG32368 & ND & $\begin{array}{l}\text { A: ubiquitous excl sal } \\
\text { gland, crop, ovary, } \\
\text { carcass; L: gut, tubule, fat } \\
\text { body; S2 cells }\end{array}$ \\
\hline CG32485 & Rab GTPase binding (i.e. membrane transport), Golgi apparatus & $\begin{array}{l}\text { ubiquitous excl L: fat } \\
\text { body, carcass }\end{array}$ \\
\hline CG32751 & $\begin{array}{l}\text { hydrolase activity, acting on carbon-nitrogen (but not peptide) bonds, in } \\
\text { linear amides, nitrogen compound metabolic process }\end{array}$ & $\begin{array}{l}\text { A: midgut, spermatheca; } \\
\text { L: gut }\end{array}$ \\
\hline Rtnl1 & $\begin{array}{l}\text { endoplasmic reticulum organization, inter-male aggressive behavior, } \\
\text { olfactory behavior, axon, cytosol, fusome, lipid particle, endoplasm ret. }\end{array}$ & ubiquitous \\
\hline CG33468 & ND & A: midgut; L: -; S2 cells \\
\hline CG33469 & ND & $\begin{array}{l}\text { A: head, gut, testis; L: sal } \\
\text { glands, gut, carcass }\end{array}$ \\
\hline CG34040 & ND & A: midgut (high); L: - \\
\hline$A r p c 3 B$ & $\begin{array}{l}\text { actin binding, structural constituent of cytoskeleton, Arp } 2 / 3 \text { complex- } \\
\text { mediated actin nucleation }\end{array}$ & $\begin{array}{l}\text { A: gut, tubule, heart, } \\
\text { testis, spermatheca; L: } \\
\text { ubiquitous excl CNS, sal } \\
\text { gland }\end{array}$ \\
\hline CG34176 & ND & $\begin{array}{l}\text { A: sal gland, gut, testis; L: } \\
\text { gut }\end{array}$ \\
\hline CG34236 & ND & $\begin{array}{l}\text { A: crop, gut; L: gut, } \\
\text { tubule, fat body; } S 2 \text { cells }\end{array}$ \\
\hline CG34253 & ND & $\begin{array}{l}\text { A: brain. Head, eye, heart, } \\
\text { fat body, testis, }\end{array}$ \\
\hline
\end{tabular}




\begin{tabular}{|c|c|c|}
\hline & & $\begin{array}{l}\text { spernatheca, carcass; L: } \\
\text { fat body }\end{array}$ \\
\hline$n u b$ & $\begin{array}{l}\text { transcription regulatory region sequence-specific DNA binding, } \\
\text { regulation of transcription from RNA polymerase II promoter, dendrite } \\
\text { morphogenesis, limb joint morphogenesis, negative regulation of } \\
\text { antibacterial peptide biosynthetic process, wing disc } \\
\text { development,ganglion mother cell fate determination, pattern } \\
\text { specification process, ventral cord development }\end{array}$ & $\begin{array}{l}\text { A: brain, head, thor gangl, } \\
\text { gut, fat body, testis; L: } \\
\text { CNS, gut, carcass }\end{array}$ \\
\hline CG42336 & ND & A: gut, testis; L: gut \\
\hline $\operatorname{serp}$ & $\begin{array}{l}\text { chitin binding, extracellular space, open tracheal system, carbohydrate } \\
\text { metabolic process, basement membrane, cytoplasm, late endosome }\end{array}$ & $\begin{array}{l}\text { A: head, eye, heart, } \\
\text { carcass; L: CNS, sla gland, } \\
\text { hindgut, trachea (high), } \\
\text { carcass (high) }\end{array}$ \\
\hline
\end{tabular}




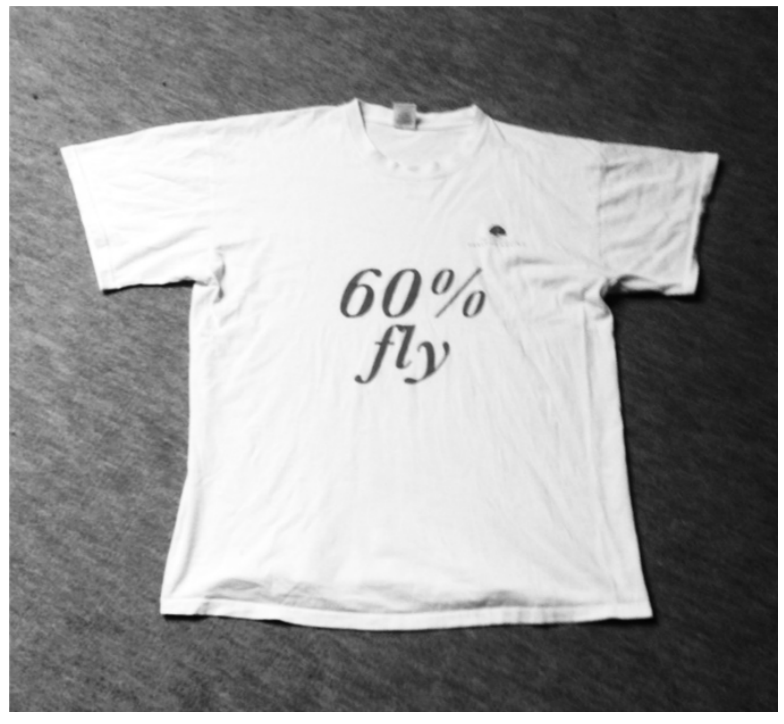

DNA sequence overlap between flies and humans... 


\section{CHAPTER VII}

LIFESPAN AND REPRODUCTION RELATIONSHIPS EXPLAIN CONSISTENT INCONSISTENCIES IN WHOLE-GENOME TRANSCRIPTION RESPONSES TO DIET IN DROSOPHILA MELANOGASTER

Jelle Zandveld

Erik van den Akker

Christina May

Joost van den Heuvel

Bas Zwaan 


\section{ABSTRACT}

Dietary restriction (DR), defined as a reduced intake of nutrients, extends lifespan in many species, but the mechanisms through which DR mediates the lifespan changes are still being explored. To date, in Drosophila multiple microarray studies have been performed as a means to uncover the genes, pathways, and processes associated with DR. The findings of these studies have, however, never been extensively compared. Here we performed a meta-analysis on microarrays in Drosophila melanogaster using three highly comparable studies and revealed large transcriptomic variations on the level of whole-genome transcription, most significant genes, and gene ontology. To explain these observations, we subjected new cohorts of flies to different adult dietary regimes and showed that agedependent reproduction patterns varied strongly; varying from a constant reproduction, to a sharp or small decrease with age. In addition, we assessed wholegenome transcription in these cohorts at different ages at which time points reproductive output was either similar or starkly different. Strikingly, agedependent reproduction determined the flies' whole-genome transcription response to diet, rather than the lifespan effect of diets per se. Therefore, age-dependent reproduction may be a likely explanation for the "consistent inconsistencies" observed between the studies used in our meta-analysis. Our findings also highlight the importance of measuring multiple life history and physiological traits when associating whole-genome transcription responses to lifespan effects of dietary restriction. 


\section{INTRODUCTION}

Dietary restriction (DR), often defined as a reduction of food intake without malnutrition (Weindruch and Walford, 1988), increases lifespan or improves health for organisms from a broad range of taxa including most model organisms, such as fungi (Jiang et al. 2000; Maas et al. 2004; Kaeberlein et al. 2005), plants (Minima et al. 2014), worms (Klass et al. 1977), fruit flies (Chippendale et al. 1993), rodents (Yu et al. 1982; Bonkowski et al. 2006), and also non-human primates (Colman et al. 2009; Mattison et al. 2012). In response to DR, many species show not only a changed lifespan, but different species increase locomotion (Huang et al. 2004; Bross et al. 2005; Overton \& Williams 2004), have a changed body composition (Skorupa et al. 2008, SolonBiet et al. 2014), increased resistance to stresses (Emran et al. 2014) and importantly, different species reduce their reproductive performance upon DR (Weindruch and Walford 1988). The exact mediating mechanisms through which DR is extending lifespan, is not yet fully understood.

\section{Genetic mechanisms of DR}

For genetic research model organisms are used to find genes or pathways involved in the DR response, and based on the principle of evolutionary conservation, with the anticipation that these pathways could have similar roles in humans (Gems and Partridge 2002; Fontana et al. 2010). Although it is a big challenge to interpret and translate these results between species, nevertheless, some genetic interventions show similar effects across species. For example, genetic interventions in the insulin-IGF signalling (IIS) and target of rapamycin (TOR) pathway extend lifespan in worms, fruit flies, and mice (Kenyon et al. 1993; Clancy et al. 2001; Bartke 2005). In fruit flies, these pathways also have a role in the lifespan response to specific components of nutrition (Grandison et al. 2009; Gronke et al. 2010; Emran et al. 2014; Zandveld et al. 2017). Also in a mice growth hormone receptor (GHR) mutant the beneficial effect of lifespan extension through DR was removed (Bonkowski et al. 2006).

\section{Whole-genome transcription between species}

Another, more systemic approach to find candidate pathways mediating the DR response, is assessing whole-genome transcription under control and DR conditions. Analysis of gene expression associations with DR phenotypes may potentially give rise to causal genes, pathways, and processes that mediate the lifespan response to DR. Whole-genome expression responses to DR in rhesus monkeys resulted in a differential expression of genes involved in cytoskeletal organisation and mitochondrial bioenergetics (Kayo et al. 2001). In mice, transcriptional patterns upon dietary-restriction showed an increased protein turnover and decreased macromolecular damage (Lee et al. 1999). And the first whole-genome transcription study in fruit flies (Pletcher $e t$ al. 2002) identified large and dynamic changing patterns with age and DR, and found the genes downregulated upon DR to play a role in cell growth, metabolism, and reproduction (Pletcher et al. 2002). These results show a seemingly species-specific effect on the processes associated with DR. But meta-analyses within groups of species, for example mammals, showed some genes, but 
mainly processes to be robustly altered due to DR (Plank et al. 2012). In another multispecies comparison for micro-array responses to DR, suggested that single gene expression is affected differently among species, but the overall physiologic effects by DR show some overlap across species (Han \& Hickey 2005).

Whole-genome transcription within fruit flies

Since the landmark fruit fly study of Pletcher et al. (2002), many more studies have been carried out that tested the effects of diet on whole-genome transcription in Drosophila (ArrayExpress: Kolesnikov et al. 2015). Although many of these showed similar effects of diet on lifespan, they differ in laboratory protocol, sometimes substantially so. For instance, gender, diet, tissue, and chronological age yielded large effects on whole-genome transcription in flies (Pletcher et al. 2002; May 2016; Lu et al. 2016; Dobson et al. 2017), but often these factors vary between studies testing the lifespan effects of DR (Piper and Partridge 2007). The strong variation in design between studies within fruit fly research, provides a unique opportunity to assesses consistency of wholegenome transcription effects within this species, extract communalities and effects of design factors, which would have the potential to serve as a default platform for inter-species comparison and translation to for instance humans. However, to our knowledge, such a systematic analysis of DR effects on whole-genome transcription responses has to date not been performed in fruit flies.

\section{This study}

We set out to examine the consistency of whole-genome transcription response to DR in Drosophila and selected three studies for whole-genome expression responses upon DR were available. By performing comparisons at the level of whole-genome transcription, on the single gene level (testing highest expressed genes (top-hits)), and on the level of processes (gene ontologies), we aim to examine the uniformity of gene transcription upon DR in fruit flies. By selecting the three most similar studies the consistencies whole-genome transcription response to DR can be verified.

In summary, we showed large inconsistencies between the three studies on all analysed levels, and hypothesized these large effects could be attributable to different reproduction patterns between the studies. In a follow-up experiment we confirmed this hypothesis and showed that agedependent reproduction patterns altered with age and strongly affected the flies' whole-genome signature upon DR. 


\section{METHODS}

Selection of whole-genome transcription studies for the meta-analysis

To find whole transcription studies on DR in Drosophila we explored the database of Array-express (Kolesnikov et al. 2015), and searched for arrays using the keywords 'aging', 'diet', 'Drosophila', and 'lifespan'. To select the most comparable studies, we used six selection criteria namely: gender, age of sampling, DR protocol, tissue, lifespan (extending) effect, and array platform. We found two studies that investigated gene expression on whole bodies in middle aged females, applying DR by diluting both sugar and yeast, and measuring expression using Affymetrix array platform 2, namely those of Doroszuk et al. (2012) and Bauer et al. (2010; hereafter to be named the Doroszuk and Bauer studies). Both studies were highly comparable with respect to the six selection criteria to another dataset acquired from our laboratory (May 2016; hereafter called the May study). Within each study, additional transcription data was available for different age classes (Doroszuk, May, and Bauer), strains (Doroszuk and Bauer), and larval food (May), but for the analyses between studies we only used data of middle age (40d, and 90\% survival for the Bauer, Doroszuk, and May studies respectively) for control strains and control larval conditions.

Micro-arrays meta-analysis

To adjust for background intensities, each separate microarray dataset was normalized using the gcrma function in R. This function converts background adjusted probe intensities to expression measures using the same normalization methods as rma (Robust Multiarray Average) (Wu \& Gentry 2017). Gene annotation was performed using the R package drosophila2.db.

\section{Follow-up}

\section{Fly husbandry}

The stock population originated from wild populations collected in 2006 from six locations across Europe. The stock population has been maintained in the laboratory for more than forty generations under standard laboratory conditions $\left(25^{\circ} \mathrm{C}, 65 \%\right.$ humidity, $12 \mathrm{~h}: 12 \mathrm{~h}$ light: dark cycle, 14-day generation time, and a standard control diet of $70 \mathrm{~g}$ yeast (Fermipan Instant Yeast Red Label), $100 \mathrm{~g}$ sugar, $20 \mathrm{~g}$ agar, $15 \mathrm{~mL}$ nipagin, and $3 \mathrm{~mL}$. propionic acid per liter of water) as described by May et al. (2015). After development for at least two generations on standard food, the follow up experiment was set up. Flies were sexed under mild $\mathrm{CO}_{2}$ anaesthesia within four hours of eclosion. Per condition, 200 unmated female flies were allocated to each of three diet treatments (see below). For 100 flies lifespan was recorded, another 100 flies were used for RNAseq analysis (see below).

\section{Diet}

Per liter, for all food types the following ingredients were added: $15 \mathrm{~g}$ agar, $3 \mathrm{ml}$ propionic acid, 30 $\mathrm{ml}$ Nipagin in 95\% ethanol. The quantities of yeast (Fermipan Instant Yeast Red Label) and sucrose varied between the food types. DR food consisted of 100 gr.l-1 sucrose and 100 gr.l-1 yeast; the 'first full feeding' condition (FF1) consisted of 200 gr.l-1 sucrose and 200 gr.l-1 yeast; and the 
'second full feeding' condition (FF2) consisted of 50 gr.l-1 sucrose and 200 gr.l-1 yeast. These diets harvest different effects on lifespan and reproduction, and take the recent insights of diet complexity into account (Lee et al. 2008; Skorupa et al. 2008). Therefore, this diet choice allowed to test effects of caloric content (DR vs FF1), changed sugar:yeast ratio's (DR vs FF2), and sugar level (FF2 vs FF1).

\section{Measuring lifespan and reproduction}

Reproduction was determined by counting the number of eggs laid in $24 \mathrm{hrs}$ per vial prior to sampling on day 12 and day 37. The lifespan phenotypes was assesses by three times per week placing flies onto fresh media and for each transfer deaths were recorded.

\section{RNA-seq}

On day 12 and day 37, total RNA was extracted using TRIzol (GIBCO) from whole bodies of between 8-10 flies per biological replicate, in total four biological replicates per condition were prepared. The NEWNExt Ultra Directional RNA Library Prep Kit for Illumina was used to process the samples. Sample preparation was performed according to the NEBNext Ultra Directional RNA Library Prep Kit for Illumina (NEB \#E7420S/L). Briefly, mRNA was isolated from total RNA using oligo-dT magneticbeads. After fragmentation of the mRNA, a cDNA synthesis was performed. This was used for ligation with the sequencing adapters and PCR amplification of the reading product. The quality and yield after sample preparation was measured with the Fragment Analyzer. Clustering and DNA sequencing using the Illumina NextSeq 500 was performed according to manufacturer's protocols. A concentration of $1.6 \mathrm{pM}$ was used. Single read sequencing was performed by GenomeScan B.V. in Leiden, the Netherlands for 16M of $76 \mathrm{bp}$ reads. Base calling and quality checks were performed with the Illumina data pipeline RTA v1.18.64 and Bcl2fastq v2.17.

The single end reads of 76 bases long were checked for quality using FastQC (Andrews 2010). Using Cutadapt (Martin 2011) reads where trimmed on quality and adapter (-q 20,-m 70). With SortMeRNA (Kopylova et al, 2012) reads were aligned to ribosomal SILVA database (Quast et al. 2013) and ribosomal RNA hits were removed. Reads were aligned to the Drosophila melanogaster genome (r6.11) using STAR (v2.5.2, Dobin et al 2013). After read alignment, only uniquely aligned reads were kept. Gene expression was quantified using HTSeq (v0.6.1, Anders et al. 2015) by exons, where all exons for a gene (all different transcripts) were unified in one synthetic transcript to be able to perform gene level gene expression analysis. The $\mathrm{R}$ ( $\mathrm{R}$ core team 2015) package limma (Smyth 2004) was used to produce normalized expression values for further analysis. Only genes that had a higher expression than 1 count in at least six samples were analyzed. Voom (Law et al. 2015) was used to normalize gene expression, with study design added as input. 


\section{Statistics}

To test lifespan effects of food treatment, a cox proportional hazard test (coxph) was performed. For reproduction, the total egg count data for each vial was summed and log transformed. An analysis of variance (Anova) was performed to test for effects the single factors and the interactions between age and dietary treatments.

For both the microarray data of the meta-analysis as well as for the RNA-seq data, per gene t-values were calculated for each diet contrast, using the Welch's t-test (equation 1). Genes from which no $\mathrm{t}$-values could be estimated, were removed from analyses.

$$
\mathrm{t}=\frac{\text { mean sample } F F-\text { mean sample } D R}{\frac{\text { stdev samples }}{\sqrt{n}}}
$$

(equation 1)

The top-hits per study or diet contrast were identified by selection of the most significant genes ( T-test, $p<0.01)$. To test for consistencies between the whole-genome transcription directions between studies or age, for each contrast a linear model $(\mathrm{lm})$ was performed on the calculate $\mathrm{t}$ values per gene. Gene ontology analysis was performed by using the online Database for Annotation, Visualization and Integrated Discovery (DAVID) (Huang et al. 2009) on the significantly, up- or downregulated genes $(T$-test, $p<0.01)$ per contrast of interest. All statistical analyses were carried out using the program $R$ ( $R$ Development Core Team 2011). 


\section{RESULTS}

After acquiring and normalization of the raw data from the Bauer, Doroszuk, and May selected micro-arrays datasets, we calculated per gene the direction of expression change and significance (T-statistics). To visualize the transcriptional variations between the studies, each plot in Figure 1 shows the $\mathrm{t}$-values per gene between all possible study contrasts.

\section{Significant but inconsistent correlations between studies}

We observed that whole-genome transcription showed two types of correlation between studies. Namely, a positive relationship between the studies of Bauer and Doroszuk $(l m, p<0.001$, Figure 1a), whereas a clear negative relationship was observed between May and Doroszuk $(l m, p<0.001$, Figure $1 b)$ as well between the datasets of May and Bauer ( $\operatorname{lm}, p<0.001$, Figure $1 c)$. Thus, while gene expression effects of DR are significantly correlated between studies, indicating consistency, the direction of this correlation is inconsistent.

These whole-genome patterns were also reflected for the identification of the most significant genes. Namely, the top hit of one study (most significant), were not shared by the other studies (Figure 1). However, among the twenty most significant genes between the study Bauer and Doroszuk one gene, CG6005, was significantly downregulated upon high food (Table S1). In correspondence to the observations for the whole-genome patterns, among the 20 most significantly affected genes in their expression upon DR diet between the studies of May and Dorosozuk (Figure 1b), two genes were significant in both studies, but showed a reversed direction of expression. The gene significantly upregulated in May et al. but down in Doroszuk et al. (CG32708) is involved in neurogenesis (Neumüller et al. 2011). The gene downregulated in May et al. but up in Doroszuk et al. (CG17119), has been implicated to be involved in transmembrane transport of amino-acids (Romero-Calderon et al. 2007). These effects show that there are correlations between studies, but the direction of change is not consistent.

\section{Implicated biological processes between the studies}

Although not much overlap was found on the single gene level, there might be similarities between the studies when looking at higher levels of biological organization, for example that of the biological processes affected by DR. Therefore, we performed a gene ontology (GO) analysis and showed on this level more consistency between the Doroszuk and Bauer studies (Table 1), which, notably, were also the studies that showed a positive relationship between their t-value distribution upon DR (Figure 1a). The GO-terms significantly up- or downregulated in the study of Bauer, showed a very similar pattern in the Doroszuk study, with the exception of the processes alternative splicing, DNA-binding, and zinc. However, when comparing the study of May with the other two studies showed that when a GO-term was significant, it had a reversed pattern, with the exception of two processes, transferase and zinc (Table 1). 
a.

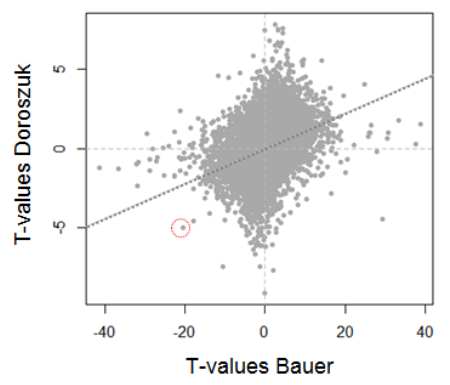

b.

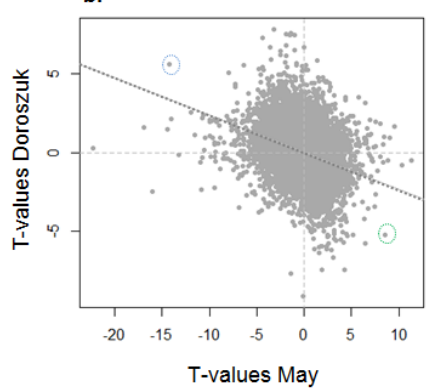

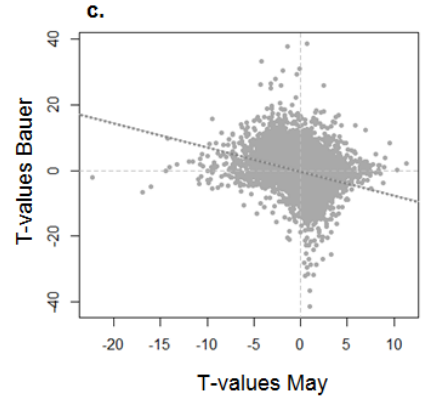

Figure 1: Effects of diet on whole-genome expression patterns between the Bauer, Doroszuk and May studies. Each graph shows a comparison between two studies for $\mathrm{t}$-values of all genes expressed between high food and DR. Each dot represents the expression of one gene; the $\mathrm{x}$-coordinate corresponds to the t-value of one study and the $y$-coordinate of the other study. (a) Comparison of t-values between the Bauer and Doroszuk studies; (b) comparison of t-values between the May and Doroszuk studies; (c) comparison of t-values between the May and Bauer studies. Genes indicated with a circle are significantly up- or downregulated between the two analysed studies, colours correspond to the genes in supplementary Table S1.

\section{Potential effects of reproduction}

Interestingly, between the studies of Bauer and May one of the inconsistent but significant enriched GO-terms was 'oogenesis'. Although this indicates a differential reproduction patterns between the studies, this could unfortunately not be verified because reproduction was not available for the study of Bauer. Moreover, previous work on whole-genome transcription responses to diet also showed enrichment for reproduction processes (Pletcher et al. 2002; Gershman et al.2007; Dobson et al. 2017; Chapter 6), and variation in reproduction variation between samples explained a large part of variation in whole-genome expression (Van den Heuvel et al. submitted; Chapter 5, this thesis). As effects within the studies in our meta-analysis indicated that different age, larval food, or genotype did not contribute substantially to whole-genome transcription responses to adult diet (Figure S1), we hypothesized that the observed consistent inconsistencies of whole-genome transcription between the Bauer, Doroszuk, and May studies, could potentially be explained by different reproduction patterns at the moment of sampling, an effect not always consistent between studies and dietary conditions (e.g. Chippindale et al. 1993; Bross et al. 2005, Van den Heuvel et al. 2014; Zandveld et al. 2017).

Age-dependent reproduction patterns on DR and full-feeding food are different.

To explore this hypothesis, we set up a new DR experiment to examine whether the whole-genome transcription response to diet is predominantly reflecting the flies' reproduction rather than the lifespan pattern. We tested the effects of lifespan and reproduction for three different DR contrasts, namely testing the effects of reduced calories; effects of a changed ratio between sugar and yeast 
(S:Y); and effects of reduced dietary sugar content. Figure 2 shows the lifespan and age-dependent reproduction results of these contrasts,

Table 1: Gene ontology analysis of most significant genes (T-statistics) upon DR between studies of Bauer et al. (2010), Doroszuk et al. (2010) and May (2016). Significant enriched GO-terms are indicated in green (upregulated) or in red (downregulated). In purple, processes that are both significantly enriched for among genes that are up- and downregulated with DR. In grey the processes that is are not significantly (ns) enriched.

\begin{tabular}{llll}
\hline Gene ontology & \multicolumn{3}{c}{ Study } \\
& Bauer & Doroszuk & May \\
\hline Metal-binding & up & up & down \\
Alternative splicing & up & down & ns \\
Hydrolase & up & ns & down \\
Lipid particle & up & ns & down \\
Membrane & up & up & down \\
Mesoderm development & up & ns & down \\
Metabolic pathways & up & ns & down \\
Oxidoreductase & up & ns & down \\
Splice variant & up & up & ns \\
Transport & up & up & ns \\
Z-disc & up & ns & down \\
Chaperone & ns & down & up \\
Cytoplasm & down & down & up \\
DNA binding & down & up & ns \\
NAD(P)-binding domain & down & ns & up \\
Neurogenesis & down & down & up \\
Oogenesis & down & ns & up \\
Phosphoprotein & down & down & ns \\
Regulation of alternative mRNA splicing & down & ns & up \\
Ribonuclease H-like domain & down & down & ns \\
Transferase & down & ns & down \\
Zinc & down & up & down \\
\hline
\end{tabular}

We observed the longest lifespan for flies on DR ( coxph, $p<0.001$, Figure 2), followed by flies on a low S:Y ratio (FF2), while the shortest lifespan was observed for flies on a high caloric diet (FF1). Notably, the flies' reproduction for these treatments interacted significantly with age (Anova, $p<0.001)$. Reproduction of flies on FF1 was highest on day 12, whereas on day 37 flies on FF2 conditions were most fecund. This shows that flies on FF1 and FF2 had a 'crossing' reproduction pattern with age. Importantly, this allowed to test the effects of reproduction patterns with age on the fly's whole-genome transcription response. 
a. Lifespan

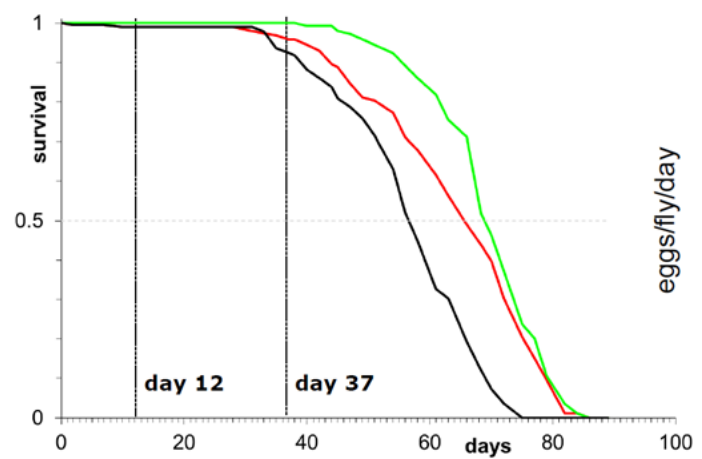

b. Age-dependent reproduction

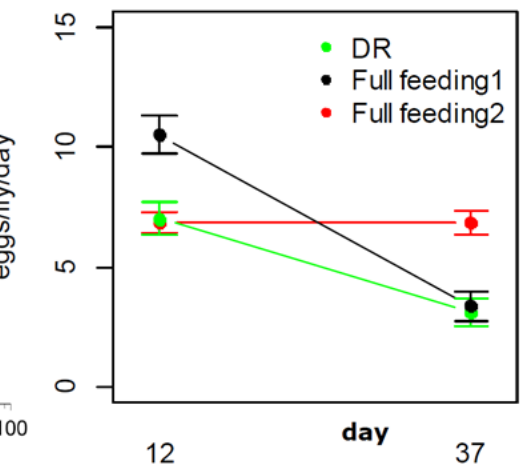

Figure 2: For the three diet treatments differing in composition or concentration, (a) the proportion survival per day (cohort size, $\mathrm{n}=100$ ), and $(\mathrm{b})$ age-dependent reproduction patterns of three different diet treatments. DR treatment contains $100 \mathrm{~g} . \mathrm{l}^{-1}$ sucrose and $100 \mathrm{~g} \cdot \mathrm{l}^{-1}$ yeast; Full-feeding 1 (FF1) contains 200

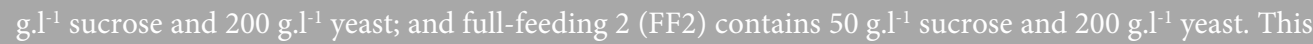
enabled testing different DR contrasts, namely between FF1 and DR the effects reduced calories; between FF2 and DR the effect of a changed ratio between sugar and yeast (S:Y); and between FF1 and FF2 the effect of reduced dietary sugar.

Age-dependent effects of whole-genome transcription

If reproduction is indeed a confounding factor in the interpretation of the whole-genome transcription observed between studies (Figure 1), we expected that the conditions showing a reversed reproduction pattern with age (FF1 and FF2, Figure 2b), should also have interactive effects with age for their whole-genome transcription response. In line with this hypothesis, conditions that do not show a differential reproduction pattern with age, will show a more consistent pattern with age.

Figure 3 shows the distribution of T-values for the three different diet contrasts. For each diet contrast, the correlation of genes up- and downregulated between day 12 and day 37 is shown. We found consistent patterns between the contrasts that did not have an inverted reproduction pattern with age, i.e. FF1-DR and FF2-DR (Figure 3a and b). However, a consistent but negative correlation was observed for the whole-genome expression response with age for FF1-FF2, the diet contrast with a reversed reproduction pattern with age $(\operatorname{lm}, p<0.001$, Figure $3 c)$. 
a. T-values FF1 - DR

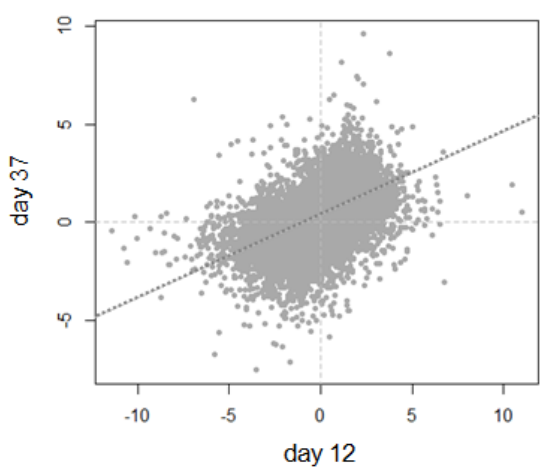

c. T-values FF1 - FF2

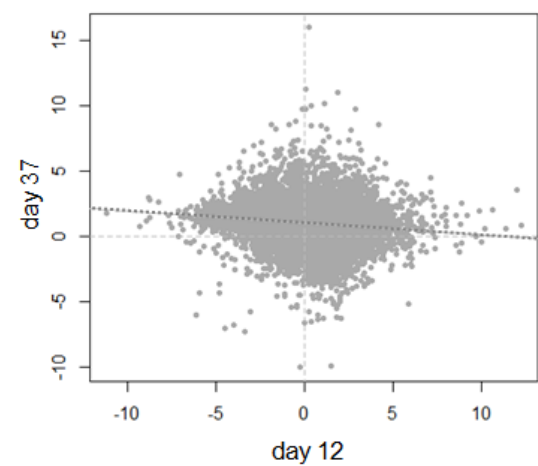

b. T-values FF2 - DR
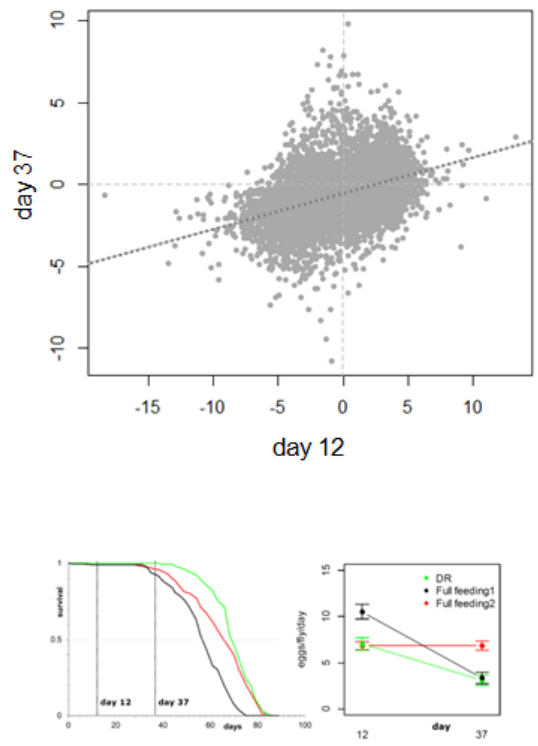

Figure 3: Effects of different dietary contrasts on whole-genome expression patterns. Graphs represent a comparison of $\mathrm{t}$-values for all genes up- and downregulated genes between the different dietary contrasts. Each dot represents the expression of one gene, with the $\mathrm{x}$-coordinate corresponding to the $\mathrm{t}$ value on day 12 and the $y$-coordinate of the same dietary contrast on day 37. (a) Comparison of t-values for DR by whole food dilution (FF1-DR); (b) comparison of t-values for a S:Y ratio change (FF2-DR); and (c) comparison of t-values for sugar dilution (FF1-FF2). For ease of interpretation, lower right panel: lifespan and reproduction effects for flies on the three diets (see also Figure 2).

\section{Significant genes}

A slightly different pattern was observed when we identified the genes significantly up- or downregulated per diet contrast for both ages ( T-test, $p<0.01)$. The largest and most consistent pattern with age was observed for the diet contrast between FF2 and DR. Here, all genes significantly up- or downregulated with diet on both ages $(n=53)$ showed the same direction of change (Table S2). For the diet contrasts FF1-DR however, seven out of the ten overlapping genes showed a consistent pattern, and, similarly, for FF1-FF2 - the diets for which reproduction reversed with age - ten out of thirteen significant genes had a consistent pattern with age (Table S2). Arguably, for this contrast, genes showing a different expression direction between the two 
ages, are consistent with the reproduction pattern, and the genes showing a similar direction with age, are consistent with the lifespan pattern observed. Following this reasoning, out of 13 significant genes between the ages, we identified ten genes up- or downregulated that were associated with the lifespan response and three with the reproduction response of this diet contrast (Table S2). Examples of significant genes associated with lifespan were Diptericin, Idgf4, and pathetic. The genes consistent with the age-dependent reproduction patterns were tango6, CG1513 and CG17239. For the list of all genes significantly up- or downregulated on both ages for each diet contrast, see supplementary Table S2.

\section{Gene ontology}

Each diet contrast was substantially enrichment for GO-terms, but not many significantly enriched GO-terms were shared between the ages (Table 2). Similar to the effects of whole-genome transcription (Figure 3), we observed substantial effects of the reversed reproduction pattern with age at the level of biological processes. The significantly enriched processes were more often reversed with age for flies with a reversed reproduction pattern with age, i.e. FF1-FF2. The other diet contrasts showed more consistent patterns (Table 2). For this diet contrast, the only process consistent with lifespan was 'alternative splicing', all other significant processes for both time points ('disulfide bond', 'peptidase S1', 'nucleotide-binding' and 'signal') were consistent with reproduction. Although for flies on the FF2-DR contrast reproduction did not switch, still we observed enriched processes reversed with age. Namely, the gene ontologies 'disulfide bond', important in protein folding/post-transcription (Sevier \& Kaiser 2002), and 'signal' were significant at both time points but in an inverted pattern (Table 2). Also for the FF1-DR contrast the process 'disulfide bond' was significantly enriched and consistent between the time points (Table 2). 
Table 2: Summary of the gene ontology analysis of most significant up- and downregulated genes (Tstatistics, $\mathrm{p}<0.01$ ) upon diet change for the different ages (day 12 and 37). The direction of each enriched GOterm is indicated in colours, namely green (upregulated) and red (downregulated).

\begin{tabular}{|c|c|c|}
\hline FF1-DR & day 12 & day 37 \\
\hline Cell junction & down & down \\
\hline Disulfide bond & down & down \\
\hline Synapse & down & down \\
\hline FF2-DR & Day 12 & Day 37 \\
\hline Disulfide bond & down & up \\
\hline Glycosidase & up & up \\
\hline $\begin{array}{l}\text { EGF-like } \\
\text {-conserved site; -like domain }\end{array}$ & down & down \\
\hline $\begin{array}{l}\text { Immunoglobulin } \\
\text {-subtype; -like domain,; -subtype 2; - like-fold; - I-set }\end{array}$ & down & down \\
\hline Signal & down & up \\
\hline FF1-FF2 & Day 12 & Day 37 \\
\hline Alternative splicing & up & up \\
\hline Disulfide bond & up & down \\
\hline $\begin{array}{l}\text { Peptidase } S 1 \\
\text {-A, chymotrypsin-type; -trypsin family, active site }\end{array}$ & up & down \\
\hline Nucleotide-binding & down & up \\
\hline Signal & up & down \\
\hline
\end{tabular}




\section{DISCUSSION}

In our meta-analysis we showed large transcriptomic variations between three highly similar studies that tested the effects of dietary restriction (DR) on whole-genome transcription. Strikingly, there were extensive correlations between gene expression effects of DR between studies, but the direction of such correlation was not consistent between studies. This may be interpreted as a low consistency in the fly's whole-genome transcription profile as a response to DR. One potential explanation for this consistent inconsistency could be correlated responses between lifespan and reproduction, and indeed we also showed that age-dependent reproduction patterns vary much between and within dietary regimes, which may very well explain inconsistencies in whole-genome transcription responses to diet, a hypothesis confirmed by the analysis of the transcriptomes of fruit flies that differ in diet induced differences in age-dependent reproduction. Although DR is often studied for its effects on lifespan, to our knowledge this is the first study that tested the extent and nature of a possible interference of reproduction in gene transcription responses to DR. Here, we identified that age-dependent reproduction is an important factor that needs to be taken into account when interpreting the fly's whole-genome transcription response to diet, especially with respect to lifespan and ageing. This underscores the importance of measuring multiple traits when assessing diets effects in fruit flies, especially for conditions when traits are known to co-vary (Van den Heuvel et al. submitted; chapter 6, this thesis)

\section{Meta-analysis studies}

Although the discrepancies between studies were observed not only on the single gene level (Figure 1) but also on the level of enriched processes (Table 1), the reversed transcription response between studies suggested an essential difference between the studies. Not only in our earlier study we observed a strong correlation between the transcriptional variation and the fly's egg laying response (Chapter 6, this thesis), but also in many other micro-array studies of diet or lifespan interventions, 'reproduction' is often enriched in gene ontology analyses of diet-inducedtranscription studies, including the ones in our meta-analysis (Pletcher et al. 2002; Gershman et al. 2007; Bauer et al. 2010; Doroszuk et al. 2012; May 2016, Dobson et al 2017; chapter 6, this thesis). Furthermore, as differential age-dependent reproduction patterns are observed upon DR and can differ substantially between studies (e.g. Chippindale et al. 1993; Bross et al. 2005, Van den Heuvel et al. 2014; Zandveld et al. 2017), we hypothesized that the (consistent) inconsistencies observed between studies could very well be explained by a different reproduction upon DR at the moment of sampling.

\section{Assessing the effect of age-dependent reproduction on whole-genome expression}

In our follow-up experiment we showed different age-dependent reproduction patterns for three dietary treatments (Figure 2). By sampling flies on two time points, this enabled us to test the effects of age-dependent reproduction on whole-genome transcription patterns. Indeed, we observed 
strong effects on whole-genome transcription signatures that were in accordance with the reproduction patterns observed (Figure 3). Ours is therefore the first study to directly show the large effects of age-dependent reproduction on the fly's whole-genome transcription pattern.

The effects of age-dependent reproduction were much reflected in the whole-genome transcription profiles (Figure 3), and also in the enriched GO processes (Table 2), but interestingly, this was not evidently the case for the overlapping genes between diet contrasts (Table S2). Namely, for the diet contrast that showed a reproduction switch with age (i.e. FF1-FF2), most were consistent between the two age classes (Table S2). Although this shows that on this level there may be more congruence, as there is still gene expression consistent with the changing reproduction pattern with age, the inconsistencies observed on this level can also be explained by the fly's reproduction pattern. Strong fluctuations of reproduction throughout life is in line with the observed dynamics with age on the transcriptomic level reported previously upon DR (Pletcher et al. 2002), but also with the reproduction dynamics of individual flies (Lu et al. 2016). Furthermore, DR effects on whole-genome transcription from fly heads and thoraces (Whitaker et al. 2014) showed much fewer significant transcripts with diet than when in a similar set up whole bodies were used (Gershman et al. 2005), which is in line with that abdominal tissues are key for reproduction and reproduction explaining a large proportion of the variation in whole-genome transcription.

The large inconsistencies observed between studies were stronger than the observed effects of agedependent reproduction in our follow-up. Although the largest transcriptomic variations between samples is most likely explained by the variations in reproduction patterns, additional traits may be contributing to the difference between studies. Important multigenic processes enriched between the studies, such as lipid metabolism and inter- and intracellular transport (Table 1), could have been differently be affected in the follow-up differently. Namely, the phenotypes strongly related to these processes also show age-dependent responses, such as locomotion (Bross et al. 2005), feeding behaviour (Wong et al. 2009), and body weight (Bross et al. 2005; Van den Heuvel et al. 2014). Despite the absence of such phenotypic data in this study, future whole-genome transcription studies, should incorporate assessing multiple phenotypes that might interfere with associating gene expression with the fly's lifespan and ageing phenotype.

\section{Decoupling lifespan and co-varying traits}

Our findings stress the importance to decouple lifespan from other traits. This can be achieved with small dietary interventions (e.g. Grandison et al. 2009; Dick et al. 2011; Chapter 4, this thesis), but has to date not been used in whole-genome transcription studies (but see: Chapter 6, this thesis). Therefore, using dietary methods that can decouple lifespan and reproduction (and other phenotypes) should improve the identification of genes truly associated with the lifespan extending effect of DR. We showed this can be achieved by dietary manipulations of sugar:yeast ratio and caloric content, that show comparable? effects on lifespan, but a different reproduction patterns with age. Namely, although some genes were significantly related with the reproduction changes, 
multiple genes were expressed consistent with lifespan, and are therefore more likely to be candidates for the lifespan changes between the two diets. Among the genes consistent with lifespan we identified genes downregulated with lifespan, e.g. Diptericin, a gene that previously has been associated with immune response (Gordon et al. 2008) and Imaginal disc growth factor 4 (Idgf4), which is involved in chitin formation and wound healing (Pesch et al. 2016). Interestingly, both the immune response and wound healing capacity are impaired upon DR in mice (Reed et al. 1996; Kristan et al. 2008), but it is not known whether these are causally related to the lifespan effects. Upregulated with lifespan was pathetic, a gene with a role in amino-acid transport and growth (Goberdhan et al. 2005), and seemingly also in neurogenesis (Lin et al. 2015). Genes that showed a consistent pattern with age-dependent reproduction were tango6, a gene with a role in protein secretion (Bard et al. 2006), CG1513, which is involved in oxysterol binding, and CG17239 that has a role in endopeptidase activity (Flybase).

We show it is possible to associate genes to the lifespan response by excluding the effects of reproduction. Although other methods can be applied to decouple lifespan and reproduction (Grandison et al. 2009; Antosh et al. 2011; Piper et al. 2017; Chapter 4 \& Chapter 5, this thesis), as the lifespan effects of diet are more consistent with age, we showed that also a differential agedependent reproduction pattern can be utilized to extract genes involved in the diet response of reproduction and ultimately lifespan.

\section{CONCLUSION}

By performing a meta-analysis among three highly similar studies, we observed consistent inconsistent effects of transcriptomic changes upon DR. Although the same lifespan extending effects were observed for each study, we hypothesized that other phenotypic changes may explain these inconsistencies and showed that age-dependent reproduction patterns can strongly affect the flies' whole-genome transcription response. This indicates that much care has to be taken in the interpretation of whole-genome transcription data when measurements of other relevant life history and physiological phenotypes are lacking. We argue that for dissecting specific groups of effector genes for the lifespan response to DR, it is crucial to measure multiple life history traits. This will improve the gene expression associations between different Drosophila studies, and is likely of strong value for further translational research from model systems to humans. 


\section{Acknowledgements}

We thank Bertha Koopmanschap for carrying out the RNA-extractions, Lidia Lopez-Gimenez for maintaining the fly populations. We thank all members of the department of Genetics, Wageningen University, and all members of the IDEAL-network (http://www.ideal-ageing.eu) for their useful discussions and comments.

\section{SUPPLEMENTAL MATERIAL}

\section{Figure S1}

Consistent transcription within studies

\section{Figure S2}

Follow-up - effects of diet within age and effect of age between different diets

\section{Figure S3}

Multi-dimensional scaling (MDS) plot for samples of follow-up experiment

\section{Table S1}

List of most significant genes (T-statistics) of meta-analysis studies

\section{Table S2}

Follow-up significant genes with same diet contrast on both ages 


\section{Figure S1}

\section{Consistent transcription within studies}
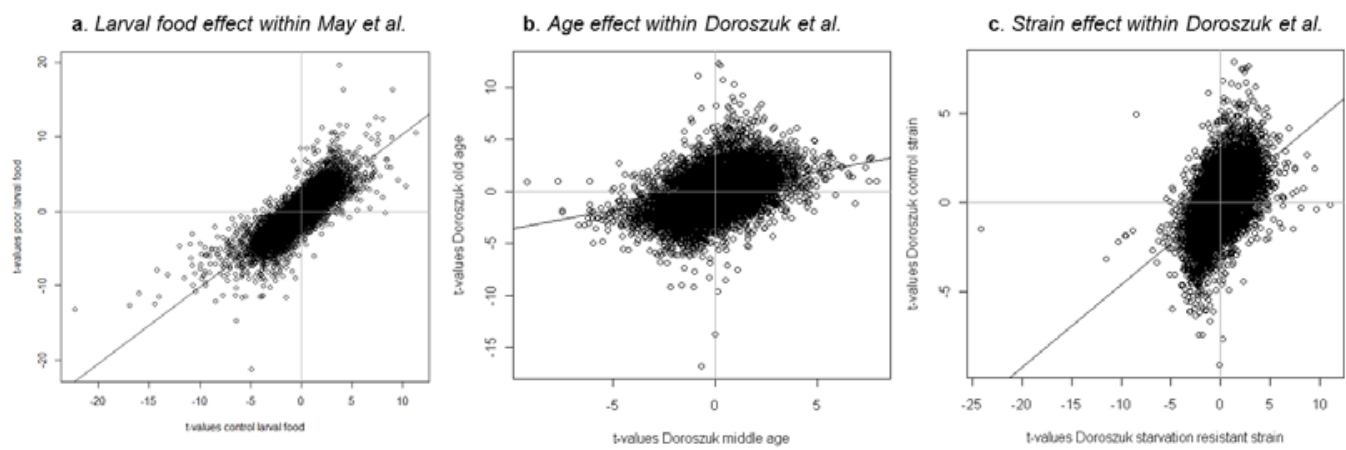

Figure S1: Comparison of t-values for all genes up- and downregulated genes between high food and DR within study shows that whole-genome transcription patterns are consistent within study. No inconsistencies observed for diet effects of flies with (a) different larval diets, i.e. control and poor larval diet (from: May et al. 2015; May et al. in prep.); (b) different ages, i.e. approx. 33d and 65d (from: Doroszuk et al. 2012); and (c) different strains, i.e. population selected for starvation resistance and a control population (from: Doroszuk et al. 2012). 


\section{Figure S2}

Follow-up - effects of diet within age and effect of age between different diets

a. $T$-values within day 12

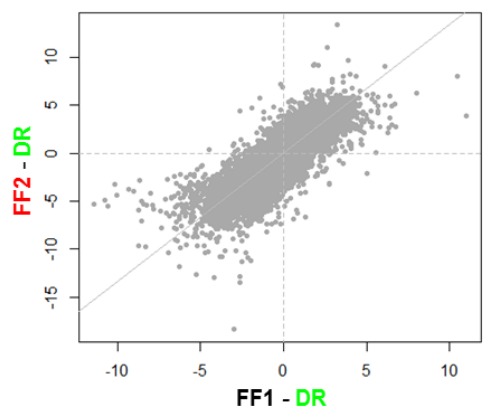

c. $T$-values within day 12

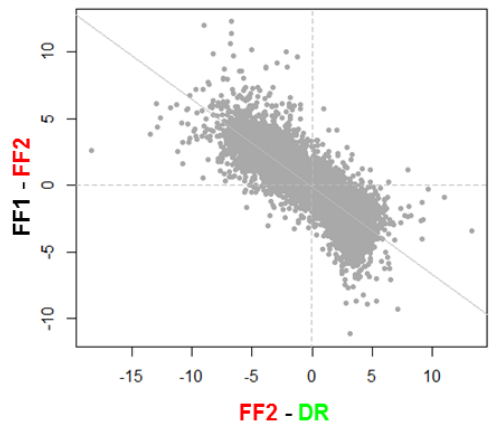

b. $T$-values within day 12
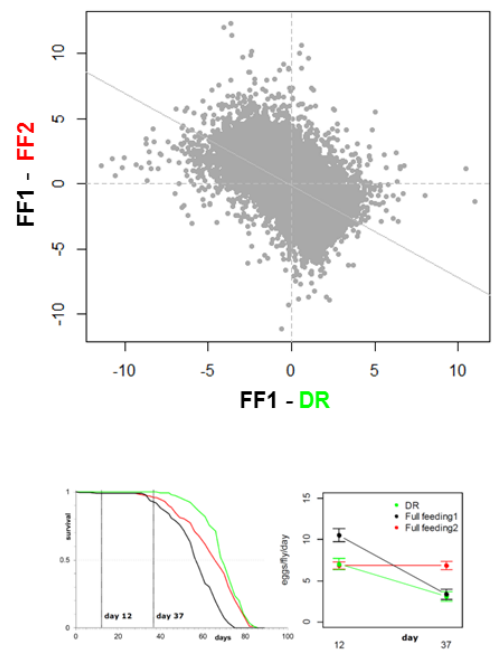

Figure S2.1: Comparison of t-values for all genes up- and downregulated genes between different diets. Whole-genome transcription patterns are not consistent with reproduction patterns within day 12 . 
a. $T$-values within day 37

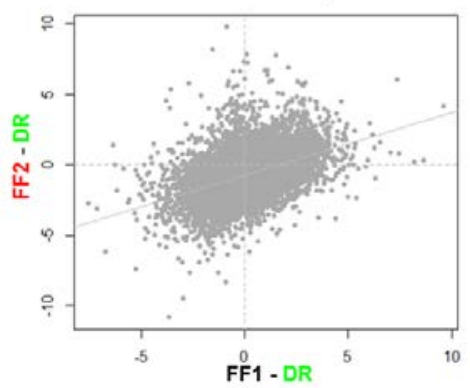

c. $T$-values within day 37

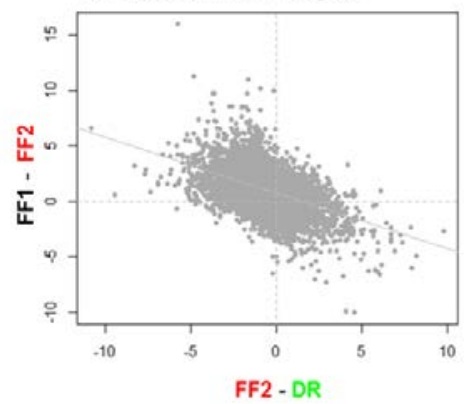

b. $T$-values within day 37
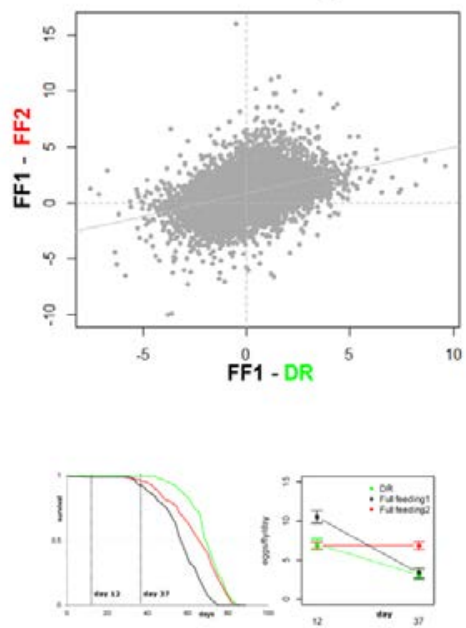

Figure S2.2: Comparison of $\mathrm{t}$-values for all genes up- and downregulated genes between different diets. Whole-genome transcription are consistent with reproduction patterns within day 37.

a. T-values between day 12 and 37

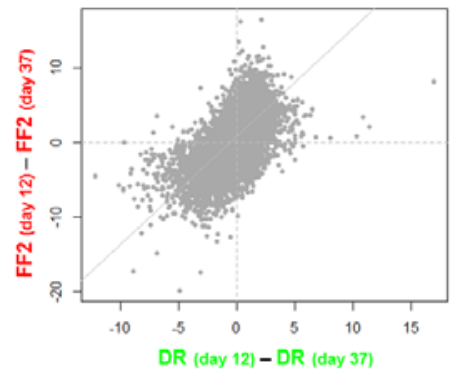

b. $T$-values between day 12 and 37

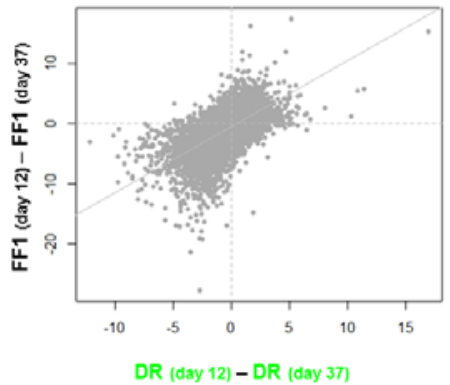

c. $T$-values between day 12 and 37

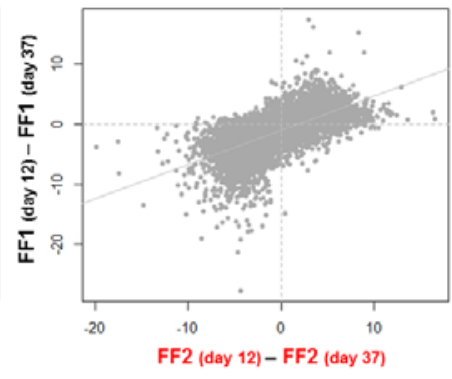

Figure S2.3: Whole-genome transcription with changing age between different diets. Age effects are consistent between diets. 

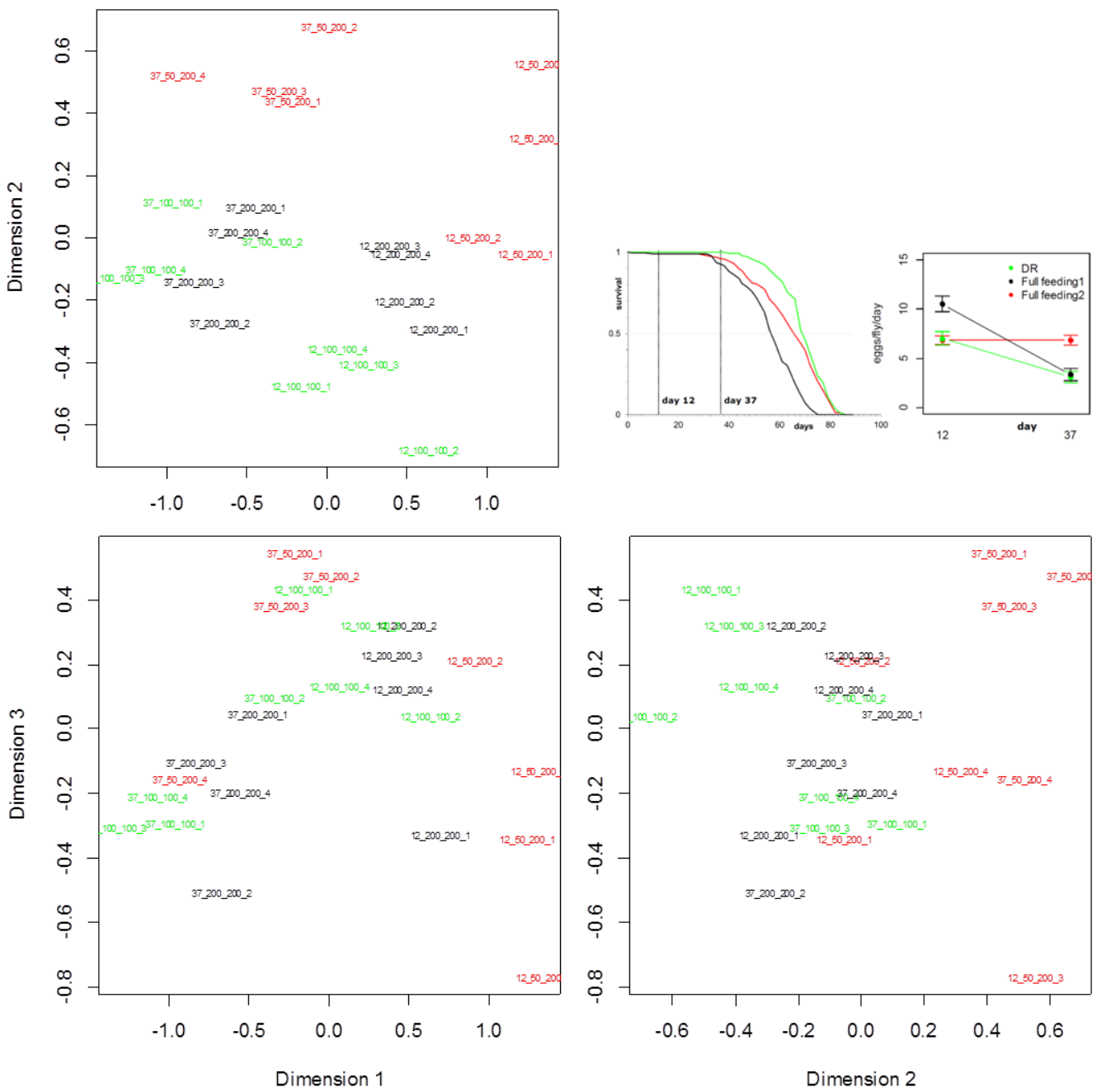

Figure S3: Multidimensional scaling plot (MDS). Distances on the plot represent coefficient of variation of expression between samples for the top genes that best distinguish the samples. Colours and coding corresponds to the day of sampling, diet treatment (presented in the top right panel) and replicate number. For instance, 12_50_200_2 corresponds to a sample taken on day 12, on the FF2 diet (50 g. $1^{-1}$ dietary sugar

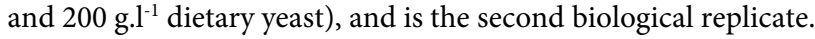




\section{Table S1}

\section{List of most significant genes (T-statistics) of meta-analysis studies}

Table S1: List of most significant genes (T-statistics) up- and down-regulated upon DR for each study in our meta-analysis. The overlapping genes are indicated with different colours and correspond to the circles surrounding the points in Figure 1.

\begin{tabular}{|c|c|c|}
\hline Up in Bauer & Up in May & Up in Doroszuk \\
\hline APP-like protein interacting protein 1(Aplip1) & CG7429 & CG17988 (Ance-3) \\
\hline CG3906 & CG32708 & CG33970 \\
\hline Sarcoplasmic calcium-binding protein $1(S c p 1)$ & CG3792 & CG11450 (net) \\
\hline Paramyosin(Prm) & CG4554 & CG12926 \\
\hline CG4827 (veil) & CG5367 & CG10911 \\
\hline Excitatory amino acid transporter 2(Eaat2) & CG15533 & $\begin{array}{l}\text { Vacuolar H[+]-ATPase } \\
\text { SFD subunit(VhaSFD) }\end{array}$ \\
\hline Ryanodine receptor(RyR) & $\begin{array}{l}\text { Something about } \\
\text { silencing } 10 \text { ortholog } \\
\text { (S. cerevisiae)(Sas10) }\end{array}$ & $\begin{array}{l}\text { Suppressor of cytokine } \\
\text { signaling at } \\
36 E(\operatorname{Socs} 36 E)\end{array}$ \\
\hline$n c R N A(C R 31781)$ & CG7968 & ETS-domain lacking(edl) \\
\hline Vacuolar H[+] ATPase 100kD subunit 5(Vha100-5) & spook(spo) & $\begin{array}{l}\text { alpha-Esterase-8(alpha- } \\
\text { Est8) }\end{array}$ \\
\hline CG3871 (Six4) & clueless(clu) & CG17119 \\
\hline Sec 23 ortholog (S. cerevisiae)(Sec23) & $\begin{array}{l}\text { Cadherin } \\
99 \text { C (Cad99C) }\end{array}$ & CG9702 \\
\hline CG8834 & $\begin{array}{l}\text { mitochondrial } \\
\text { ribosomal protein } \\
\text { S35(mRpS35) }\end{array}$ & $\begin{array}{l}\text { Down syndrome cell } \\
\text { adhesion molecule } \\
1(\text { Dscam } 1)\end{array}$ \\
\hline Actin $79 B(A c t 79 B)$ & CG12128 & $\begin{array}{l}\text { Vacuolar } H[+] \text { ATPase } \\
44 k D \text { subunit(Vha44) }\end{array}$ \\
\hline CG32984 & $\begin{array}{l}\text { Signal recognition } \\
\text { particle protein } \\
14(\text { Srp14) }\end{array}$ & CG31288 \\
\hline Jonah $66 \mathrm{Ci}($ Jon66Ci) & CG15481 (Ski6) & Myosin 28B1(Myo28B1) \\
\hline Organic cation transporter(Orct) & CG7381 & wishful thinking(wit) \\
\hline CG9889 (yellow-d) & CG6147 (Tsc1) & $\begin{array}{l}\text { Serine-arginine protein } \\
\text { kinase at } 79 D(S r p k 79 D)\end{array}$ \\
\hline beta-Mannosidase(beta-Man) & CG4825 & $\begin{array}{l}\text { ATP synthase, beta } \\
\text { subunit(ATPsynbeta) }\end{array}$ \\
\hline \multirow[t]{2}{*}{ inactivation no afterpotential D(inaD) } & $\begin{array}{l}\text { Niemann-Pick type C- } \\
2 e(N p c 2 e)\end{array}$ & CG8630 \\
\hline & CG30185 & CG15255 \\
\hline
\end{tabular}




\begin{tabular}{|c|c|c|}
\hline Down in Bauer & Down in May & Down in Doroszuk \\
\hline CG2218 & CG1942 & CG32708 \\
\hline$B E A F-32$ & Cytochrome b5(Cyt-b5) & $\begin{array}{l}\text { female sterile (1) } \\
\operatorname{Nasrat}\left(f_{s}(1) N\right)\end{array}$ \\
\hline Partner of Y14 and Mago(Pym) & CG9044 & CG4743 \\
\hline coiled(cold) & CG9360 & CG14823 \\
\hline CG12728 & $\begin{array}{l}\text { Glutathione S transferase } \\
\mathrm{O} 2(\mathrm{GstO} 2)\end{array}$ & $\begin{array}{l}\text { multiple ankyrin repeats single } \\
\text { KH domain(mask) }\end{array}$ \\
\hline Replication Protein A 70(RpA-70) & $\begin{array}{l}\text { NADH dehydrogenase } \\
\text { (ubiquinone) B22 } \\
\text { subunit(ND-B22) }\end{array}$ & abnormal wing $\operatorname{discs}(a w d)$ \\
\hline CG10222 & CG3962 (Keap1) & $\begin{array}{l}\text { Lysophosphatidylcholine } \\
\text { acyltransferase(LPCAT) }\end{array}$ \\
\hline CG1603 & $\begin{array}{l}\text { Ecdysone-induced protein } \\
28 / 29 k D(\text { Eip71CD) }\end{array}$ & Cullin 3(Cul3) \\
\hline CG17977 & CG17119 & $\begin{array}{l}\text { Iron regulatory protein } 1 B \text { (Irp- } \\
1 B)\end{array}$ \\
\hline CG34261 & $\begin{array}{l}\text { peroxisomal Multifunctional } \\
\text { enzyme type } 2(M f e 2)\end{array}$ & eIF2B-delta \\
\hline CG15435 & CG4572 & CG4849 \\
\hline spindle $B(s p n-B)$ & $\begin{array}{l}\text { Cytochrome P450- } \\
4 e 2(\text { Cyp } 4 e 2)\end{array}$ & $\begin{array}{l}\text { bicoid-interacting protein } \\
\text { 3(bin3) }\end{array}$ \\
\hline Mediator complex subunit 21 (MED21) & $\begin{array}{l}\text { alpha/beta hydrolase } \\
\text { 1(Hydr1) }\end{array}$ & CG1349 (dj-1beta) \\
\hline CG18809 & CG31743 & CG17726 \\
\hline Heat shock protein $26(H s p 26)$ & CG31955 & CG6005 \\
\hline Cyclin $C(C y c C)$ & CG7470 & Nucleoporin $153 k D(N u p 153)$ \\
\hline CG8950 & pumpless(ppl) & CG31457 \\
\hline CG6005 & CG13315 & $\begin{array}{l}\text { Threonyl-tRNA } \\
\text { synthetase(Aats-thr) }\end{array}$ \\
\hline CG12520 & CG18609 & CG31381 \\
\hline CG14667 & cabut(cbt) & CG10646 \\
\hline
\end{tabular}




\section{Table S2}

\section{Follow-up significant genes with same diet contrast on both ages}

Table S2: Genelist of significantly up- or downregulated genes $(p<0.01)$ for a specific diet contrast on both day 12 and day 37

\section{Diet} contrast
Flybase no

Gene name
T-value day

12
T-value day

37

FF1-DR

$\begin{array}{llll}\text { FBgn0000273 } & \begin{array}{l}\text { Protein kinase, cAMP-dependent, } \\ \text { catalytic subunit 1(Pka-C1) }\end{array} & -4.922 & 4.008 \\ \text { FBgn0002571 } & \begin{array}{l}\text { Maltase A3(Mal-A3) } \\ \text { Octopamine-Tyramine }\end{array} & 4.992 & 4.891 \\ \text { FBgn0004514 } & \begin{array}{l}\text { receptor(Oct-TyrR) } \\ \text { FBgn0004861 }\end{array} & -4.522 & 4.167 \\ \text { FBgn0024321 } & \text { CG8524(NK7.1) } & -4.200 & -5.208 \\ \text { FBgn0027525 } & \text { CG7686 } & -5.572 & -5.651 \\ \text { FBgn0030120 } & \text { CG17440) } & -5.787 & -6.736 \\ \text { FBgn0030589 } & \text { CG9519 } & 4.414 & 4.772 \\ \text { FBgn0038282 } & \text { defective proboscis extension } & -6.919 & 6.310 \\ \text { FBgn0259984 } & \text { response 9(dpr9) } & -3.866 & -5.266 \\ & \text { kuzbanian(kuz) } & 4.184 & 4.582\end{array}$

FF2 - DR

FBgn0000038
FBgn0000046
FBgn0003248
FBgn0004102
FBgn0004919
FBgn0005624
FBgn0005677
FBgn0013348

FBgn0019957

FBgn0020378

FBgn0027562

FBgn0030102

FBgn0030156

FBgn0031258

FBgn0031627 nicotinic Acetylcholine Receptor beta1(nAChRbeta1)

Actin 87E(Act87E)

$-6.435$

$-4.887$

Rhodopsin 2(Rh2)

$-7.576$

$-3.968$

ocelliless(oc)

goliath (gol)

Posterior sex combs(Psc)

dachshund(dac)

$-10.866$

$-4.199$

$-4.916$

$-6.748$

$-4.642$

$-4.199$

5.636

4.151

$-5.574$

$-4.535$

Troponin C at 41C(TpnC41C)

$-9.615$

$-5.835$

$\mathrm{NADH}$ dehydrogenase

(ubiquinone) $42 \mathrm{kDa}$

$-3.852$

$-4.714$

subunit(ND-42)

CG1343 (Sp1)

$-5.704$

$-5.177$

CG10345

$-5.175$

$-4.781$

CG12119

$-3.945$

$-4.067$

CG15247

$-7.118$

$-4.369$

CG4297

$-4.672$

$-3.949$

CG15630) 


\begin{tabular}{|c|c|c|c|}
\hline FBgn0031858 & CG17378 & -4.825 & -4.973 \\
\hline FBgn0032897 & CG9336 & -5.533 & -4.848 \\
\hline FBgn0032899 & CG9338 & -5.513 & -5.241 \\
\hline FBgn0033446 & CG1648 & -4.723 & -4.075 \\
\hline FBgn0033980 & CG10245 (Сyp6a20) & -4.575 & -4.626 \\
\hline FBgn0034070 & CG8403 (SP2353) & -7.751 & -4.233 \\
\hline FBgn0034199 & Growth-blocking peptide(Gbp) & -4.462 & -4.586 \\
\hline FBgn0034509 & $\begin{array}{l}\text { Odorant-binding protein } \\
57 c(\text { Obp57c) }\end{array}$ & -6.343 & -4.119 \\
\hline FBgn0034538 & CG16799 & -5.447 & -5.094 \\
\hline FBgn0035086 & CG12851 & -4.425 & -5.851 \\
\hline FBgn0035639 & CG5537 & 4.299 & 5.679 \\
\hline FBgn0036553 & CG17027 & -5.334 & -4.112 \\
\hline FBgn0036882 & CG9279 & -4.217 & -5.174 \\
\hline FBgn0037163 & lazaro(laza) & -4.341 & -4.433 \\
\hline FBgn0037989 & ATPase $8 B(A T P 8 B)$ & -4.648 & -3.742 \\
\hline FBgn0038181 & CG9297 & -5.292 & -4.693 \\
\hline FBgn0038282 & $\begin{array}{l}\text { defective proboscis extension } \\
\text { response } 9(d p r 9)\end{array}$ & -5.583 & -7.366 \\
\hline FBgn0039075 & CG4393 & -4.975 & -5.786 \\
\hline FBgn0039102 & Spatzle-Processing Enzyme(SPE) & -4.379 & -4.943 \\
\hline FBgn0039600 & CG1646 & -4.605 & -4.272 \\
\hline FBgn0050008 & CG30008 & -4.876 & -3.961 \\
\hline FBgn0051414 & CG31414 & -6.020 & -3.803 \\
\hline FBgn0051665 & weary $(w r y)$ & -3.785 & -4.926 \\
\hline FBgn0051675 & CG31675 & -13.464 & -4.834 \\
\hline FBgn0051676 & CG31676 & -4.564 & -5.128 \\
\hline FBgn0052311 & CG33484 (zormin) & -4.853 & -3.943 \\
\hline FBgn0052432 & CG32432 & -6.322 & -4.005 \\
\hline FBgn0053329 & Serine-peptidase 212(Sp212) & -4.502 & -3.784 \\
\hline FBgn0053493 & CG33493 & -10.157 & -5.069 \\
\hline FBgn0085237 & CG34208 & -6.666 & -4.350 \\
\hline FBgn0259150 & CG42265 & -4.601 & -4.978 \\
\hline FBgn0259219 & CG42319 & -4.451 & -4.381 \\
\hline FBgn0259748 & CG42397 & -4.414 & -4.959 \\
\hline FBgn0259994 & CG42492 & -9.585 & -4.878 \\
\hline FBgn0260660 & Multiplexin $(M p)$ & -5.604 & -5.003 \\
\hline FBgn0263219 & $\begin{array}{l}\text { Down syndrome cell adhesion } \\
\text { molecule } 4 \text { (Dscam4) }\end{array}$ & -5.182 & -4.474 \\
\hline FBgn0264503 & CG43902 & -4.823 & -4.249 \\
\hline FBgn0264606 & CG43955 (Fife) & -7.091 & -4.540 \\
\hline
\end{tabular}

FF1-FF2

$\begin{array}{llll}\text { FBgn0004240 Diptericin(Dpt) } & 3.917 & 5.257\end{array}$ 


\begin{tabular}{llll} 
FBgn0026415 & $\begin{array}{l}\text { Imaginal disc growth factor } 4 \\
\text { (Idgf4) }\end{array}$ & 4.220 & 8.496 \\
FBgn0032494 & $\begin{array}{l}\text { CG5945 } \\
\text { Transport and Golgi } \\
\text { FBgn0032728 }\end{array}$ & 4.654 & 4.348 \\
FBgn0033463 & CG1513 & -7.038 & 4.701 \\
FBgn0036007 & Pathetic(path) & -4.264 & 5.201 \\
FBgn0039611 & CG14528 & -6.132 & -5.993 \\
FBgn0042186 & CG17239 & 4.567 & 5.591 \\
FBgn0053110 & CG33110 & 5.873 & -5.225 \\
FBgn0053653 & Calcium-dependent secretion & 4.144 & 5.945 \\
& activator(Cadps) & 7.107 & 4.477 \\
FBgn0086443 & Asparaginyl-tRNA & & \\
FBgn0259994 & CG4thetase(Aats-asn) & -4.783 & -4.365 \\
FBgn0262717 & CG43161 (Skeletor) & 5.463 & 4.160 \\
& & 4.472 & 4.513 \\
\hline
\end{tabular}




\section{De ballade van de dood}

\author{
K. Mijnderts, $H$. Jekkers
}

Er was eens een koning machtig en groot Die had slechts één vijand en dat was de dood Waarom moest de dood toch zijn leven bederven Waarom was ie zo bang, zo bang om te sterven De koning ontbood toen al zijn geleerden Die te paard en per koets aan het hof arriveerden "Môge geleerden", zei de koning beleefd "Ik zit met een vraag waar niemand antwoord op heeft"

De jongste geleerde een ijdele snaak Riep: "Vraagt u maar Sire, vraagt u maar raak! Wilt u soms weten hoeveel sterren er zijn?" Of hoe zwaar al het zand weegt van de grote woestijn? Of hoe de belasting massaal wordt ontdoken? Of hoe..." maar toen werd hij abrupt onderbroken "Wel nee", zei de koning een tikje afwezig "Waarom gaan we dood? Kijk dat houdt mij bezig!" Niet één der geleerden had zo'n vraag verwacht "Al sla je me dood!" zei de jongste heel zacht De oudste geleerde nam toen het woord En zei: "Sire, sommige mensen worden vermoord, anderen komen per ongeluk om, maar de meesten sterven van ouderdom" De koning zei kribbig: "Ja dat wist ik allang, maar wat is de dood, waarom ben ik zo bang?" De knapste geleerde zei: "Mag ik soms even? Misschien moet $u$, Sire, met de dood leren leven." De koning sprong op, zijn woede was groot en hij schreeuwde: "Ik eis een antwoord! Wat is de dood?" Toen sprak een geleerde met veel fantasie:

"Zal ik eens vertellen Sire, hoe ik dat nu zie?"

"De dood komt je halen, de dood raakt je aan, dus de dood moet in levende lijve bestaan! We moeten hem vangen, dan zijn we eraf Leve het leven! Weg met het graf!"
Toen kreeg de koning een schitterend plan: Hij zei: "Ik ken een stokoude, doodzieke man. $\mathrm{Hij}$ heeft schat ik nog maar een uurtje te gaan, dus de dood komt hem halen, de dood komt eraan. We bouwen een glazen kooi om zijn bed en de deur wordt uitnodigend opengezet. Is de dood eenmaal binnen, op weg naar z'n prooi, dan sluiten we snel de deur van de kooi."

Aldus werd besloten, men ging aan de slag en de dood werd gevangen, nog diezelfde dag. Somber en treurig zat hij achter het glas alsof hij een levend museumstuk was. Nog nooit was het volk zo gelukkig geweest, jaren en jaren vierde men feest.

Maar op den duur ging het feesten vervelen en ging men gevaarlijke spelletjes spelen. Men sprong van torens, in diepe ravijnen, men stoeide met leeuwen en met wilde zwijnen. Men dronk liters en liters vergiftigde wijn en voerde wat oorlog gewoon voor de gein. En niemand ging dood, geen mens ging verloren, maar er werden wel steeds meer baby's geboren Het werd alsmaar drukker, men kreeg het benauwd, er werden zelfs mensen de zee ingedouwd En honderd jaar later was de lol er vanaf en ging men weer verlangen naar de rust van het graf

De koning dacht: goed ik ben niet meer bang, maar ik vind alles zo saai en ik regeer al zo lang. Opnieuw riep hij toen de geleerden bijeen en zei: "Wat een ellende! Waar moet dat heen?" De knapste geleerde, inmiddels zo'n drie eeuwen oud zei: "Bevrijd toch de dood! Want zo gaat het fout." Maar de jongste geleerde zei: "Jahaa, wie laat hem los? Wie de deur opendoet is als eerste de klos!" De koning stond op en zei theatraal:

"Laat mij het maar doen. Gegroet allemaal! Mijn angst voor de dood is nu wel genezen. Ik heb geloof ik meer van het eeuwige leven te vrezen!"

Hij schreed naar de kooi, machtig en groot En stierf in de armen van de gretige dood "Leve de dood!" riep het volk dolgelukkig En ze leefden nog lang en stierven gelukkig 


\section{CHAPTER VIII}

\section{SYNTHESIS}

On the genetic mechanisms of nutrient-dependent lifespan and reproduction

Jelle Zandveld 


\section{SYNTHESIS}

In this thesis I aimed to improve the understanding of the genetic mechanisms mediating nutrientdependent ageing. By using two model organisms for ageing research, namely the fungus Podospora anserina and the fruit fly Drosophila melanogaster, I investigated genetic effects on diet responses using a variety of experimental approaches. In Chapter 2 I tested the effects of a genetic intervention in the insulin signalling pathway (dilp2-3,5 knockout mutants) that extended the flies' lifespan on all nine different assay diets, but whether the knockout changed the lifespan response to diet was dependent on the diet regimen considered. In Chapter 3, I examined for the same range of diets the effects of the knockout on the gene expression dynamics of up- and downstream of the insulin signalling pathway and showed a diet-dependent upregulation of dilp6 in the mutants that could maintain the insulin-IGF signalling (IIS) responses to dietary yeast. Furthermore, natural genetic variation in strains of Podospora anserina was measured and analysed in Chapter 4 to explore the variation for the lifespan response to a gradient of seven glucose levels. Strains showed ample variation in different response parameters for the lifespan change, which allowed to decouple the diet effects on lifespan and reproduction, i.e. traits that typically co-vary with diet change. In Chapter 5, natural genetic contrasts were generated through experimental evolution of Drosophila populations. Here, I showed that adaptation to distinct nutritional conditions was not accompanied with a consistent change of the fly's lifespan diet response to these nutrients. In Chapter 6 I subjected flies to a fluctuating diet throughout life. Here I observed strong fluctuations of the whole-genome transcription response, and the treatments assessed made it again possible to separately associate genes to the reproduction or lifespan response to diet. In my last experimental chapter, Chapter 7, I further focussed on the fly's whole-genome transcription responses, by performing a meta-analysis on Drosophila studies that had investigated the association between the effects of diet on lifespan whole-genome and gene expression. Results between studies were 'consistently inconsistent", which I revealed to be potentially causes by the large role of reproduction variation in studies of whole-genome transcription in females.

In this final Chapter 8 I will synthesize the findings across this thesis and will further elaborate on how they may affect our understanding of genetic mechanisms of the lifespan response to diet. I will also discuss future direction for genetic research on lifespan and diet.

\section{Considering Nutrient Space In Drosophila And Podospora Lifespan}

I showed that upon a triple knockout of $d i l p 2,-3$ and -5 flies reproduced less, but lived much longer irrespective of diet. Because this effect was observed for all nine diets including the lifespan-bydiet optimum, I concluded that dilp2-3,5 knockout flies extend lifespan in a manner that is largely independent of diet. Without applying a broad diet range, such a strong conclusion would not have been possible as important genotypic effects and interactions would have been overlooked. The importance of using multiple diets was also demonstrated for the first fly mutant in the IIS pathway, chico, a gene encoding for the insulin receptor substrate. The original paper using a single 
diet was reported an increased lifespan upon chico knockout (Clancy et al. 2001), whereas this genetic intervention actually generated a shift of the flies' lifespan response to diet uncovered by using a range of diets (Clancy et al. 2002, Figure 1). My work and this example showed that dependent on diet concentration, different conclusions can be drawn whether or not a genetic intervention extends lifespan or changes the diet response. So, only by sampling a broad range of food types, the actual genotypic effects on lifespan will be revealed (reviewed by: Mair \& Dillin 2008; Flatt 2014)

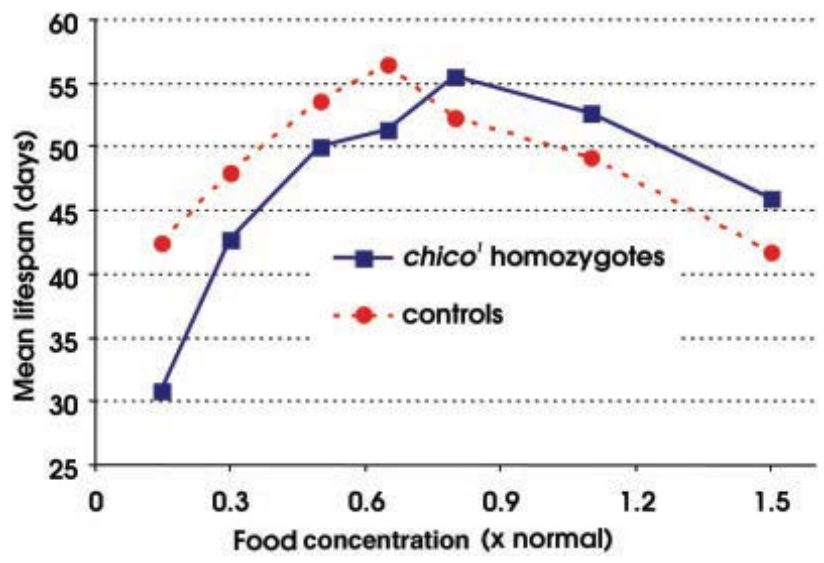

Figure 1: Figure from Clancy et al. (2002). Interaction between dietary restriction by food dilution and reduced IIS by chico deletion in the determination of mean lifespan. These effects of chico deletion would be perceived as a lifespan increase when only tested on high diets concentrations, but perceived as a lifespan decrease on the lower concentrations.

Moreover, the Geometric Framework (GF) builds on this notion by considering nutrition to be a multiple-dimensional space in which different dietary components are represented by separate axes and any phenotypic response can be plotted on another axis (Simpson and Raubenheimer 1993). This implementation of increased diet complexity is proposed to improve the understanding of how genotypes affect the lifespan response to certain diet dimensions (Piper et al. 2011). I verified this by showing diet-specific effects of the dilp2-3,5 knockout that would not have been measured when a smaller part of nutrient space was employed. The general effect of the triple knockout was a strong lifespan increase and a small shift of the optimal lifespan to higher sugar levels. For different nutrient dimensions this effect ranged from reducing the diet response, to an enhanced or even unchanged response upon diet change, similar to what was proposed by Piper et al. (2011; see also: Chapter 1, Figure 2). Different laboratories vary in the food concentration and composition applied when testing effects of DR (Piper \& Partridge 2007), and different interpretations have emerged about whether or not IIS is involved in Drosophila's lifespan 
response to diet (Clancy et al. 2002; Min et al. 2008; Grandison et al. 2009; Gronke et al. 2010; Naif Al-Saud et al. 2015; Dobson et al. 2017). I showed that the interactive effects between IIS and diet were highly dependent on the nutrient dimension under consideration, which provides an explanation of the observed inconsistencies between studies. When in future studies a broader range of nutrient space is sampled, this increases the nutritional overlap between studies, and should therefore reveal fewer contradictory outcomes. A starting point for this could be my observations that the lifespan change upon dietary sugar in the mutants suggests a mediating role of IIS for the lifespan response to changing sugar. As the response to changing yeast concentration has not been changed by the dilp2-3,5 knockout, this suggests no mediating role of IIS in yeast responses of lifespan.

In Chapter 3, I performed an expression analysis of remaining dilps upon knockout of dilp2-3,5 and observed a changed expression pattern upon changing yeast of the remaining head-dilp, dilp6, which may be in conflict with the interpretation from phenotypic data that the lifespan response to yeast is not mediated via IIS (Chapter 2). Consistent with reports on dilp upregulation upon single and multiple dilp knockout (Broughton et al. 2008; Gronke et al. 2010) and dilp downregulation upon dilp6 overexpression (Bai et al. 2012), this finding suggests a compensatory mechanism among dilps. Compensatory action and redundancy within broad gene families are more often observed and suggested to be evolved for maintenance of accurate signalling for important processes and cope with random fluctuations (Kafri et al. 2009). This is in line with dynamic environmental fluctuations that flies are likely to experience in the wild, and the role of dilps in fitness-related traits, such as development time, body composition, lifespan, and reproduction (Gronke et al. 2010; Okamoto et al. 2009; Slaidina et al. 2009, Chapter 2).

The compensatory effects of dilp6 were, however, only observed on the highest yeast level, which again shows that the broad range of diets covered was essential to acquire this insight. I showed that the maintained dilp activity upstream in the IIS pathway was consistent with the retained downstream diet effects on $4 e-B P$ expression in the fly bodies. However, this is no evidence that dilp6 alone fully compensates to maintain the lifespan response to yeast in dilp2-3,5 mutants. Much transcriptional change occurs upon knockout of master regulators of lifespan and reproduction, for example when ablating cells producing insulin (Buch et al. 2008), or when ablating cells producing juvenile hormone (Yamamoto et al. 2013). These large transcriptional changes suggest that a total new transcriptional homeostasis is reached through compensatory gene expression changes that occur throughout the whole-genome (e.g. Buch et al. 2008; Yamamoto et al. 2013).

Moreover, the multiple components dietary yeast consists of, i.e. fatty-acids, sugars, vitamins and importantly, amino-acids, would also strongly support that multiple pathways should be employed for the flies' to response to this combined variety of nutrients. The Target Of Rapamycin (TOR) pathway, an interconnected pathway with IIS, is also shown to be involved in dietary responses in flies (Kapahi et al. 2004; Bjedov et al. 2010) and other organisms (Kaeberlein et al. 2005; Wei et al. 
2008; Henderson et al. 2006). That rapamycin treatment reduced the effects of amino-acid supplementation to DR in flies (Emran et al. 2014), shows TOR has an important role in the diet response specific to amino-acids. Together, this suggests that compensatory mechanisms through IIS, but also through TOR and possibly other pathways could maintain the flies' lifespan response to yeast.

Recently, much effort was put in developing a fully synthetic diet for Drosophila (Piper et al. 2014), which would offer new and exciting opportunities in parsing out detailed aspects of diet complexity. Implementation of this synthetic diet revealed the significance of amino-acid balance for Drosophila lifespan and reproduction patterns (Soultoukis 2016; Piper et al. 2017). This demonstrates that for studying the subtle effects of diet, using a synthetic medium can be extremely helpful for further dissecting the exact genetic effects on the lifespan response to diet.

In Chapter 4 I used a synthetic diet to measure detailed effects on the lifespan response of changing the glucose concentration in small steps in a variety of wild-derived strains of the fungus Podospora anserina.. While all strains showed an increased lifespan upon glucose restriction, much variation was observed in the response of the different strains, an effect that would not have been revealed if lifespan was measured only for the four highest concentrations. Although the effects of glucose restriction on lifespan have been investigated previously in our laboratory (Maas et al. 2004; Van Diepeningen et al. 2008; Van Diepeningen et al. 2010a, Van Diepeningen et al. 2010b), to date no tests were performed in any system on natural strains using such a broad range of diets. Many of the studies on DR in natural strains previously performed in other species typically applied only two diets. The effects of DR across natural populations and across species suggests there is much variation whether DR extends lifespan (Harper et al. 2006; Sutphin \& Kaeberlein 2008; Terzibazi et al. 2009; Nagakawa et al. 2012; Stastna et al. 2015), but these effects were measured on just a few diets, a method shown to be potentially problematic for interpretation of DR effects by genotype (e.g. Figure 1). To my knowledge, only one study (on Drosophila) measured the lifespan response of multiple natural strains on a broad range of diets and showed clear differences across natural populations for the optimal diet for lifespan, but interestingly, for all tested populations a lifespan extension was achieved through DR (Metaxakis \& Partridge 2013).

Similarly, this may also be the case for genetic effects other than those shaped by evolutionary forces in natural populations. For example in the screening of single mutants in yeast (Schleit et al. 2013) but also for recombinant inbred lines in mice (Liao et al. 2010), DR responses ranged from a large extension to strong reductions in lifespan. Although these variable responses can provide valuable insights for the identification of genes involved in the lifespan response to DR, the experimental design could not depict more detailed effects, i.e. whether DR always extends lifespan, or whether some genotypes had a shifted nutrient response, while others may show a true differential response to nutrient restriction (i.e. Figure 1, Chapter 1). Exactly these effects were tackled as well in Chapter 4, by measuring lifespan on multiple glucose concentrations and fitting 
a non-linear model to the lifespan data. This allowed the estimation of two response parameters, i.e. the threshold and slope of the reaction norm. A different threshold of the lifespan response causes a shifted pattern along any diet-axis (e.g. demonstrated in Figure 1), possibly resulting from a changed sensitivity to diet. A different slope, however, causes a reduced ability to change to an altered dietary condition, and could possibly result from a blocked capacity for lifespan to respond to diet change. Strains varied strongly for both these parameters, which demonstrated ample natural genetic variation for the different components of the lifespan response to glucose.

Also, both parameters correlated with lifespan measured at $2 \%$ glucose levels, the commonly used control diet in effects on ageing in Podospora (Maas et al. 2004; Van Diepeningen et al. 2009). This correlation of lifespan was much weaker for the slope of the response than for the threshold. But despite the weak correlation between lifespan and the slope of the response, it does show at least partly an overlapping mechanism between general lifespan and the lifespan response to diet. Candidate mechanisms consistent with this link would be processes involved in mtDNA integrity. Much Podospora ageing research has been performed on mitochondrial effects (Osiewacz 2002; Lorin et al. 2006) and interestingly, also natural strains that contained the $p A L 2-1$ plasmid, a plasmid that seems to destabilize the mitochondrial genome, also showed a strongly reduced response to glucose and interestingly, a short lifespan (Van Diepeningen et al. 2008). So to investigating mitochondrial function in strains that differ in the slope of the response may reveal further insight in the mechanism through which Podospora can extend lifespan upon glucose restriction. For example, in reciprocal crosses between Podospora strains maternal effects between strains that differ in the response slope, could indicate a strong mitochondrial effects in mediating this response.

A changed DR threshold towards higher diets without changing of the pattern will be interpreted as a lifespan increase on higher diets, as illustrated in Figure 1. A lifespan shift is regarded to be less relevant to depict the exact mechanisms mediating lifespan extension through DR, as it does not change the response profile, but rather the sensitivity to diet (Mair \& Dillin 2008; Flatt 2014). Although different Podospora strains showed different thresholds of the lifespan response, interestingly, these differences were in some cases accompanied with a similar reproduction pattern. This in principle allows us to disentangle genes involved in lifespan from those in reproduction. As shown in Chapter 6 and Chapter 7, for gene expression associations, other processes than lifespan often interfere with lifespan upon diet change. When reproduction and lifespan can be decoupled, such as in this example for Podospora, will certainly improve the quality of efforts to associate genes to the lifespan effects. The broad range of diets applied in Podospora has thus proven not only to reveal detailed genetic effects on the diet response, it is also demonstrated to be a useful tool for disentangling lifespan and reproduction. 


\section{Diet Responses And Life-History Adaptations}

The previously mentioned study (Metaxakis \& Partridge 2013) observed interesting patterns in (natural) populations of Drosophila, namely that the only laboratory strain had an overall longer lifespan and higher reproduction, but also a shifted lifespan response with diet compared to the natural strains (Figure 2). Similarly, the longest-lived natural strain was also longer-lived and more fertile and showed its optimal lifespan on a relatively richer diet. These observations suggests that laboratory adaptations (or pre-adaptation) resulted in a longer lifespan, an enhanced reproduction, but also a changed the DR threshold on the diet conditions tested in the laboratory. This notion was explored in Chapter 5, where I experimentally evolved populations on three nutritionally distinct food types. I showed that the fly's reproduction response to diet has been changed through experimental evolution, but there were no consistent changes for the lifespan response to diet. The broad range of diets on which lifespan was measured, indicates this is not an artefact of diet choice, but rather that the connection between reproduction and lifespan upon diet change is decoupled through evolutionary change. This thus indicates that lifespan and reproduction are not necessarily (genetically) connected upon evolutionary adaptations to different diets. Rather, the strong lineage-specific effects suggest a neutrally evolving lifespan response. But as other life-history traits were also evolved in response to the experimental evolution conditions, the improved reproductive capacity is potentially achieved through a whole suit of life-history changes.
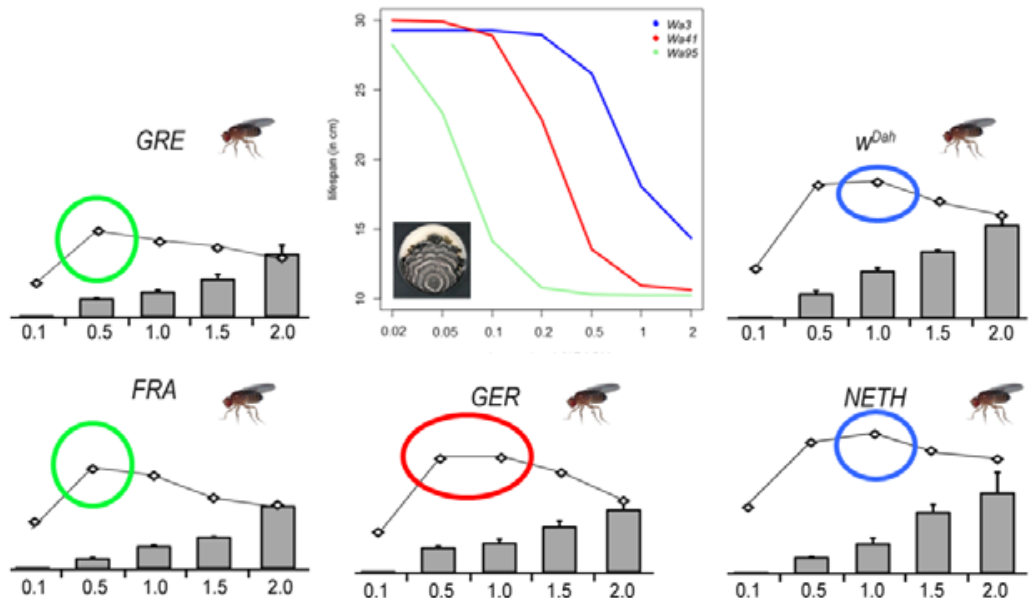

Figure 2: The lifespan response of Podospora and the lifespan and reproduction responses for Drosophila to a broad range of diets. The relationship between lifespan and DR threshold in natural strains of Podospora (top central) seem to have an equivalent in the response of natural strains in Drosophila, indicated by the similar colours. Drosophila short-lived strains seem to require a more severe restriction to increase lifespan upon DR. wDah, a lab-adapted strain, shows a longer lifespan and changed lifespan optimum to higher diet levels. The Drosophila lifespan and reproduction plots are adapted from Metaxakis \& Partridge (2013). 
In Chapters 2, 4, and 5 I have shown the importance of applying the insights of nutritional geometry to different cases of genetic change. Extending this approach of nutritional geometry to more genotypes and even to multiple species, may reveal genotype by diet responses previously not reported or unnoticed for assays on a limited range of nutrient conditions. For example, recent tests in individuals of long-lived families (and their partners) for their response to a diet and activity intervention, revealed no interactive effects of the long-lived background and the health response (Van de Rest et al. 2015). But the observed interaction between long life and diet response parameters reported in this thesis for Podospora, seems to be present in Drosophila as well (Metaxakis \& Partridge 2013, Figure 2), and the data indicates that to investigate subtle interactions - such as between familial lifespan and diet response - actually needs more diets to assess in any study system, thereby including humans as well. Depicting the detailed genotypic effects on the slope or threshold of the lifespan response to diet, in this thesis shown for diet alterations in Drosophila and Podospora, is a helpful tool for identifying key players in the lifespan response to nutrients and through the possibility of decoupling the lifespan and reproduction response.

\section{Co-Varying Traits}

Although it has been often reported that the lifespan change upon DR is accompanied by a reduction of reproduction, I showed in this thesis through measuring natural populations of Podospora or experimental evolution populations in Drosophila that the decoupling of these traits is possible. This can be extremely useful for linking the life history response to diet to genetic mechanisms for the individual traits, for example through gene expression analysis. In Chapters 6 and 7, I indeed focussed on the lifespan-reproduction interrelationship and found methods to decouple genes consistently associated with lifespan changes from those for reproduction. In Chapter 6 the importance of measuring reproduction was demonstrated, not only because the dynamic changes in reproduction observed in our previous study (Van den Heuvel et al. 2014) were shown to be accompanied by a complete switch of the flies' whole-genome transcription profile, but mainly by the strong correlation between the first principal component of variation and the fly's reproduction at the moment of sampling. This indicates the presence of a wellorchestrated reproduction response for flies that experience a fluctuating diet, and suggests an adaptive response to a strongly varying environment in nature. Importantly, it also shows that gene expression responses to diet in flies are chiefly reflecting the fly's reproduction status. The strong correlation to reproduction for many genes observed in Chapter $\mathbf{6}$ allowed me to isolate groups of genes independently associated to reproduction or lifespan. By ignoring the genes correlated to reproduction, it was possible to extract a core set of genes with a stronger association to the lifespan effects measured. Moreover, by performing a co-expression network analysis one network showed much overlap with this core set. The highly connected gene in this network ("hub") could therefore be an important mediator of the lifespan response to diet. These results showed that by measuring both lifespan and reproduction, it is possible to identify genes involved in either one of these processes. Furthermore, both the enriched sets of core reproduction and core lifespan genes 
showed a large overlap with significant gene sets of other whole-genome transcription studies on lifespan change (Pletcher et al. 2002; Yamamoto et al. 2013; Doroszuk et al. 2012). This implies that in these studies many genes previously associated with the lifespan effects, have also a role in the reproduction response.

A similar effect was demonstrated in Chapter 7, where clear inconsistent patterns were observed between three studies (selected for their similarity in experimental approach) that investigated whole-genome transcription responses upon DR. As the lifespan effects were consistent between the studies, one would expect a considerable level of similarity between the studies, but this was not observed on the whole-genome level, neither for the most significant genes, nor for the GO analysis. The large effects of reproduction observed in Chapter 6, but also the enrichment of reproduction in many other studies (e.g. Pletcher et al .2002; Bauer et al. 2010; Doroszuk et al. 2012, Dobson et al. 2017), and the non-random relationship between the inconsistent expression responses ("consistent inconsistencies"), suggested that a different reproduction pattern could account for this effects. I set out to verify this hypothesis by a follow-up experiment and showed that age-dependent reproduction strongly affected the whole-genome transcription response. In this experiment, the gene expression related to the reproduction patterns are unlikely to yield mediators of the lifespan effects between the treatments. But the genes that do not relate to reproduction but do with lifespan change, are more likely lifespan candidates. Again, this stresses the importance to measure effects of reproduction when performing whole-genome transcription, not only in dietary restriction, but also in any other intervention, especially when traits are known to co-vary. Strong sex-dependent effects have been observed upon diet change, not only for lifespan, but also reproduction (Magwere et al. 2004; Jensen et al. 2015), which is consistent with a strong altered whole-genome transcription profile upon DR between the sexes (May 2016, thesis). Males may show fewer physiological effects upon DR, such as the strong effect of reproduction in females, and could therefore be useful for the association of gene expression to lifespan change. Testing the consistency of whole-genome transcription response for male Drosophila, is then expected to reveal more consistent results between studies. However, whole genome transcription in males could reflect other large physiological effects that strongly co-vary with the lifespan effects, for example locomotion (Bross et al. 2005) and fat storage (Skorupa et al. 2009). So the experiments performed in Chapter 6 and 7 in this thesis indicate whole genome transcription largely reflects reproduction patterns in female flies, and showed that multiple phenotypes should be measured in order to decouple genes involved in lifespan of in other (correlated) processes.

In this thesis I showed several examples of how to decouple lifespan and reproduction responses. Namely, through, 1) combining small nutritional steps with natural genetic variation in Podospora (Chapter 4), 2) experimental evolution that affects nutrient-dependent reproduction without consistent lifespan effects (Chapter 5), 3) measuring reproduction in flies experiencing a fluctuating adult diet (Chapter 6), and 4) measuring age-dependent reproduction changes 
(Chapter 7). These methods have proved their significance for connecting (life-history) traits specific effects to genes, pathways, and processes using whole-genome transcription analyses as in this thesis (e.g. Chapters 6 and 7), in providing opportunities for future investigation by decoupling lifespan and reproduction (e.g. Chapters 4 and 5), and also encourages a re-evaluation of past performed research on whole-genome transcription in response to diet (Chapters 6 and 7).

\section{Genetic Mechanisms Of Nutrient-Dependent Lifespan}

For an overview of the insights into the genetic mechanisms of nutrient-dependent lifespan acquired in this thesis, I summarize my findings in Figure 3 by using the concept of the Y-model (De Jong \& Van Noordwijk 1992). The Y-model is a resource acquisition and allocation model in which the connection between diet, genetics, and life-history is conceptualized?. In short, increased nutrient acquisition will lead to more investment in both lifespan and reproduction, while the (genetic) allocation strategy can change the relative contribution of the nutrients to either of these. Crucially, the model predicts that (genetic) variation for resource acquisition results in positive correlations between traits, while such variation for resource allocation will result in a negative correlation between lifespan and reproduction (so called "trade-offs"). I have extended upon the model illustration by showing the regulatory level of mediating the lifespan or reproduction response to diet by positioning genes in Figure 3.

In Chapter 2 I showed that a dilp2-3,5 knockout resulted a strong lifespan increase that was irrespective of diet, but also a strong reduction of the flies fecundity. This shows a strong role of IIS in lifespan with pleiotropic effects on reproduction, so seemingly through an resource allocation shift (Figure 3a). For the involvement of IIS in diet responses, I showed the knockout reduced the lifespan decrease with increasing dietary sugar, and therefore IIS is at least partly involved in the lifespan response to sugar (Figure 3a). The effects of IIS on mediating the effects of yeast, have not totally been resolved, compensation could partly maintain this response within IIS. Insights in DR studies investigating the role of TOR signalling in the diet response, shows however much potential of TOR to have a mediating role in the lifespan response to yeast (Figure 3a).

Podospora natural strains showed much variation for the lifespan response to glucose, even on the lower food levels where no reproduction was observed. This suggests genetic mechanisms that are involved in lifespan variation, but not interconnected with the reproduction response (Figure 1b). Also, the observed correlation between the lifespan response and longevity suggests an overlapping mechanism between these two processes. A potential candidate discussed would be mitochondrial functioning, but has yet to be examined.

Experimental evolution in Drosophila resulted in local adaptation, i.e. increased reproduction on the flies' evolutionary food type, but had no consistent effect the lifespan response to diet (Figure $3 \mathrm{c}$ ). The large variations between replicates suggests the lifespan response is neutrally evolved, and not indirectly affected by the changed reproduction response. Similar to Podospora, this suggests that the adaptive genetic mechanisms that increased the fly's reproduction, are not necessarily interconnected with lifespan change. 
We have observed that whole-genome transcription response to diet are largely reflecting the flies' reproduction pattern, and identify therefore much fewer transcripts related to lifespan. But network analysis identified highly connected genes (hubs) for lifespan (network 3 ) and on different levels of regulation for reproduction (network 1, 2 and 4) (Figure 3d).

a

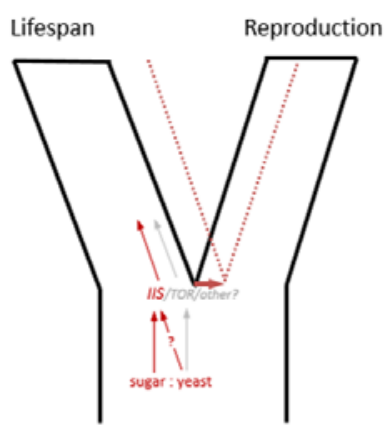

c

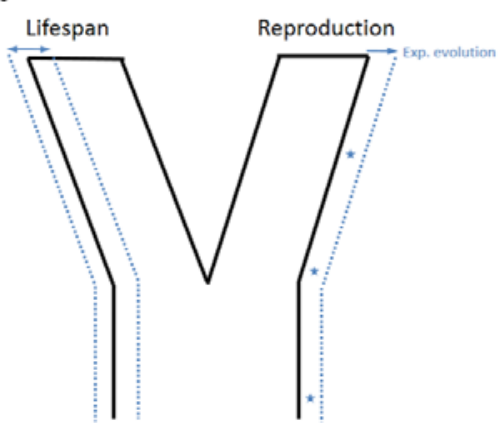

b

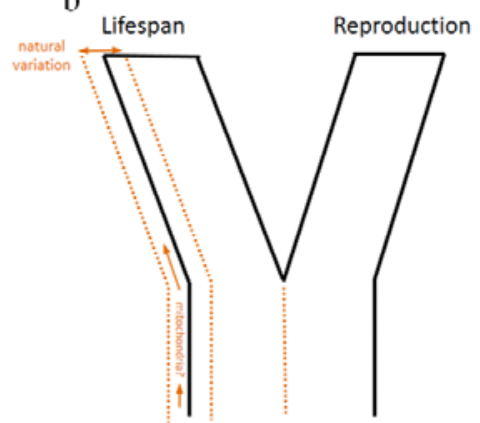

d

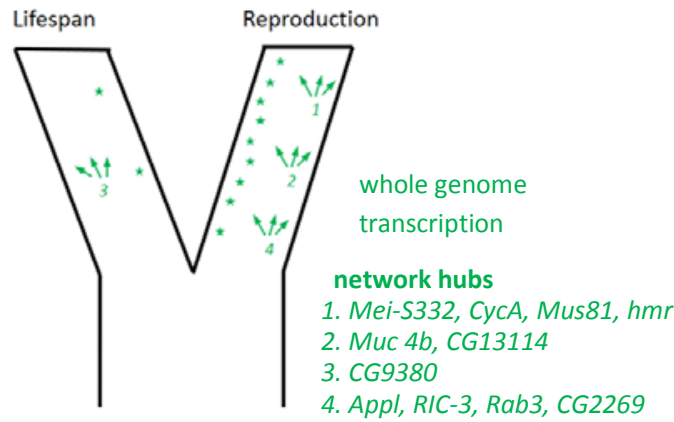

Figure 3: Overview of the findings in this thesis about the mediating genetic mechanisms of nutrientdependent lifespan. Diet intake can be allocated to reproduction or lifespan, increased diet acquisition (wider basis) can lead to an increase of both or either of the traits. Along the route from diet intake to trait, the genetic mechanisms can mediate the response of diet. (a) Reduced IIS extends lifespan and strongly reduces reproduction (thick red arrow, allocation shift). But IIS is also involved in the diet response to dietary sugar. The yeast response is potentially partly mediated by IIS (red arrow '?'), but more likely combined with other pathways such as TOR (grey arrows). (b) Podopsora natural variation showed much variation for the lifespan response to diet, even upon glucose restriction, when no reproduction was recorded (dotted orange lines). Mitochondrial functioning may partly affect the lifespan response to diet observed between natural strains (orange arrow). (c) Experimental evolution in Drosophila increased reproduction, without changing the lifespan response to diet, possibly by larger acquisition and not by an allocation shift (blue line). (d) Whole-genome transcription response to diet identifies mainly genes involved in reproduction and much fewer in lifespan (green stars). Network analysis identified highly connected genes (hubs) on different levels of regulation for both lifespan (network 3) and reproduction (network 1,2 and 4). 


\section{CONCLUDING REMARKS}

In my thesis I have shown that for a proper interpretation of genetic effects on the lifespan response to diet, a broad range of food types should be implemented in the study design. By applying the recent insights of nutritional geometry to a variety of test cases for genetic effects, i.e. a knockout mutant, natural genetic variation, and genetic change by experimental evolution, I have shown the potential of this framework for identifying important genotype-by-diet interactions and for obtaining more insight into how lifespan can be extended by diet manipulation. Furthermore, I showed that through different analysis tools and dietary methods it is possible to decouple lifespan and reproduction in two model systems. I have shown the value of this decoupling for the association of gene expression to lifespan, and also showed the potential of using natural genetic variation for decoupling lifespan and reproduction changes to diet.

Future work should focus on extending the approach of nutritional geometry to more genotypes and species, which may identify new genotypic effects on the lifespan response to diet that to date have not yet been considered. What I see especially as highly promising is to further exploiting systems in which decoupling between traits is possible, for example by choosing dietary conditions for which phenotypic responses between traits contrast. 


\section{REFERENCES}

- $\quad$ Ackermann, M., Bijlsma, R., James, A. C., Partridge, L., Zwaan, B. J., \& Stearns, S. C. (2001). Effects of assay conditions in life history experiments with Drosophila melanogaster. Journal of Evolutionary Biology, 14(2), 199-209.

- $\quad$ Al Saud, S. N., Summerfield, A. C., \& Alic, N. (2015). Ablation of insulin-producing cells prevents obesity but not premature mortality caused by a high-sugar diet in Drosophila. Proceedings of the Royal Society of London B: Biological Sciences, 282(1800), 20141720.

- $\quad$ Alic, N., Andrews, T. D., Giannakou, M. E., Papatheodorou, I., Slack, C., Hoddinott, M. P., ... \& Partridge, L. (2011). Genome-wide dFOXO targets and topology of the transcriptomic response to stress and insulin signalling. Molecular systems biology, 7(1), 502.

- Antosh, M., Whitaker, R., Kroll, A., Hosier, S., Chang, C., Bauer, J., ... \& Helfand, S. L. (2011). Comparative transcriptional pathway bioinformatic analysis of dietary restriction, Sir2, p53 and resveratrol life span extension inDrosophila. Cell cycle, 10(6), 904-911.

- Aruna, S., Flores, H. A., \& Barbash, D. A. (2009). Reduced fertility of Drosophila melanogaster Hybrid male rescue (Hmr) mutant females is partially complemented by $\mathrm{Hmr}$ orthologs from sibling species. Genetics, 181(4), 1437-1450.

- Austad, S. N. (1989). Life extension by dietary restriction in the bowl and doily spider, Frontinella pyramitela. Experimental gerontology, 24(1), 83-92.

- Baayen, R. H. (2008). Analyzing linguistic data: A practical introduction to statistics using R. Cambridge University Press.

- Bai, H., Kang, P., \& Tatar, M. (2012). Drosophila insulin-like peptide-6 (dilp6) expression from fat body extends lifespan and represses secretion of Drosophila insulin-like peptide-2 from the brain. Aging cell, 11(6), 978-985.

- Baldal, E. A., Brakefield, P. M., \& Zwaan, B. J. (2006). Multitrait evolution in lines of Drosophila melanogaster selected for increased starvation resistance: the role of metabolic rate and implications for the evolution of longevity. Evolution, 60(7), 1435-1444.

- $\quad$ Bard, F., Casano, L., Mallabiabarrena, A., Wallace, E., Saito, K., Kitayama, H., ... \& Perrimon, N. (2006). Functional genomics reveals genes involved in protein secretion and Golgi organization. Nature, 439(7076), 604-607.

- Bartke, A. (2005). Minireview: role of the growth hormone/insulin-like growth factor system in mammalian aging. Endocrinology, 146(9), 3718-3723.

- Bauer, J., Antosh, M., Chang, C., Schorl, C., Kolli, S., Neretti, N., \& Helfand, S. L. (2010). Comparative transcriptional profiling identifies takeout as a gene that regulates life span. Aging (Albany NY), 2(5), 298-310.

- Bento, G., Ogawa, A., \& Sommer, R. J. (2010). Co-option of the hormone-signalling module dafachronic acidDAF-12 in nematode evolution. Nature, 466(7305), 494-497.

- Berrar, D. P., Downes, C. S., \& Dubitzky, W. (2003). Multiclass cancer classification using gene expression profiling and probabilistic neural networks. In Proceedings of the Pacific symposium on biocomputing (Vol. 8, pp. 5-16).

- $\quad$ Bjedov, I., Toivonen, J. M., Kerr, F., Slack, C., Jacobson, J., Foley, A., \& Partridge, L. (2010). Mechanisms of life span extension by rapamycin in the fruit fly Drosophila melanogaster. Cell metabolism, 11(1), 35-46.

- Blüher, M., Kahn, B. B., \& Kahn, C. R. (2003). Extended longevity in mice lacking the insulin receptor in adipose tissue. Science, 299(5606), 572-574.

- Bolstad, B. M., Irizarry, R. A., Åstrand, M., \& Speed, T. P. (2003). A comparison of normalization methods for high density oligonucleotide array data based on variance and bias. Bioinformatics, 19(2), 185-193.

- $\quad$ Bonkowski, M. S., Rocha, J. S., Masternak, M. M., Al Regaiey, K. A., \& Bartke, A. (2006). Targeted disruption of growth hormone receptor interferes with the beneficial actions of calorie restriction. Proceedings of the National Academy of Sciences, 103(20), 7901-7905.

- Bross, T. G., Rogina, B., \& Helfand, S. L. (2005). Behavioral, physical, and demographic changes in Drosophila populations through dietary restriction. Aging cell, 4(6), 309-317. 
- $\quad$ Broughton, S. J., Piper, M. D., Ikeya, T., Bass, T. M., Jacobson, J., Driege, Y., ... \& Partridge, L. (2005). Longer lifespan, altered metabolism, and stress resistance in Drosophila from ablation of cells making insulin-like ligands. Proceedings of the National Academy of Sciences of the United States of America, 102(8), 3105-3110.

- $\quad$ Broughton, S., Alic, N., Slack, C., Bass, T., Ikeya, T., Vinti, G., ... \& Partridge, L. (2008). Reduction of DILP2 in Drosophila triages a metabolic phenotype from lifespan revealing redundancy and compensation among DILPs. PloS one, 3(11), e3721.

- $\quad$ Broughton, S. J., Slack, C., Alic, N., Metaxakis, A., Bass, T. M., Driege, Y., \& Partridge, L. (2010). DILPproducing median neurosecretory cells in the Drosophila brain mediate the response of lifespan to nutrition. Aging cell, 9(3), 336-346.

- Bruce, K. D., Hoxha, S., Carvalho, G. B., Yamada, R., Wang, H. D., Karayan, P., ... \& William, W. J. (2013). High carbohydrate-low protein consumption maximizes Drosophila lifespan. Experimental gerontology, 48(10), 1129-1135.

- $\quad$ Buch, S., Melcher, C., Bauer, M., Katzenberger, J., \& Pankratz, M. J. (2008). Opposing effects of dietary protein and sugar regulate a transcriptional target of Drosophila insulin-like peptide signaling. Cell metabolism, 7(4), 321-332.

- Burger, J., \& Promislow, D. E. (2004). Sex-specific effects of interventions that extend fly life span. Science's SAGE KE, 2004(28), pe30.

- Carey, J. R., Liedo, P., Harshman, L., Zhang, Y., Müller, H. G., Partridge, L., \& Wang, J. L. (2002). Life history response of Mediterranean fruit flies to dietary restriction. Aging cell, 1(2), 140-148.

- CBS Statline, 2017, http://statline.cbs.nl

- Chamseddin, K. H., Khan, S. Q., Nguyen, M. L., Antosh, M., Morris, S. N. S., Kolli, S., ... \& Bauer, J. H. (2012). takeout-dependent longevity is associated with altered Juvenile Hormone signaling. Mechanisms of ageing and development, 133(11), 637-646.

- Chapman, T., Trevitt, S., \& Partridge, L. (1994). Remating and male-derived nutrients in Drosophila melanogaster. Journal of Evolutionary Biology, 7(1), 51-69.

- Chippindale, A. K., Leroi, A. M., Kim, S. B., \& Rose, M. R. (1993). Phenotypic plasticity and selection in Drosophila life-history evolution. I. Nutrition and the cost of reproduction. Journal of Evolutionary Biology, 6(2), 171-193.

- Clancy, D. J., \& Kennington, W. J. (2001). A simple method to achieve consistent larval density in bottle cultures. Drosoph. Inf. Serv, 84, 168-169.

- Clancy, D. J., Gems, D., Hafen, E., Leevers, S. J., \& Partridge, L. (2002). Dietary restriction in long-lived dwarf flies. Science, 296(5566), 319-319.

- Clancy, D. J., Gems, D., Harshman, L. G., Oldham, S., Stocker, H., Hafen, E., ... \& Partridge, L. (2001). Extension of life-span by loss of CHICO, a Drosophila insulin receptor substrate protein. Science, 292(5514), $104-106$.

- Colman, R. J., Anderson, R. M., Johnson, S. C., Kastman, E. K., Kosmatka, K. J., Beasley, T. M., ... \& Weindruch, R. (2009). Caloric restriction delays disease onset and mortality in rhesus monkeys. Science, 325(5937), 201204.

- Colombani, J., Andersen, D. S., \& Léopold, P. (2012). Secreted peptide Dilp8 coordinates Drosophila tissue growth with developmental timing. Science, 336(6081), 582-585.

- Cooper, T. M., Mockett, R. J., Sohal, B. H., Sohal, R. S., \& Orr, W. C. (2004). Effect of caloric restriction on life span of the housefly, Musca domestica. The FASEB journal, 18(13), 1591-1593.

- Curtsinger, J. W. (2013). Late-life fecundity plateaus in Drosophila melanogaster can be explained by variation in reproductive life spans. Experimental gerontology, 48(11), 1338-1342.

- De Jong, G., \& Van Noordwijk, A. J. (1992). Acquisition and allocation of resources: genetic (co) variances, selection, and life histories. The American Naturalist, 139(4), 749-770.

- Dennis, G., Sherman, B. T., Hosack, D. A., Yang, J., Gao, W., Lane, H. C., \& Lempicki, R. A. (2003). DAVID: database for annotation, visualization, and integrated discovery. Genome biology, 4(9), R60. 
- Dick, K. B., Ross, C. R., \& Yampolsky, L. Y. (2011). Genetic variation of dietary restriction and the effects of nutrient-free water and amino acid supplements on lifespan and fecundity of Drosophila. Genetics research, 93(04), 265-273.

- Dillin, A., Crawford, D. K., \& Kenyon, C. (2002). Timing requirements for insulin/IGF-1 signaling in C. elegans. Science, 298(5594), 830-834.

- Dobson A.J., Ezcurra M., Flanagan C.E., Summerfield A.C., Piper M.D.W., Gems D., Alic N. (2017). Nutritional Programming of Lifespan by FOXO Inhibition on Sugar-Rich Diets, Cell Reports, 18, 299-306

- Doroszuk, A., Jonker, M. J., Pul, N., Breit, T. M., \& Zwaan, B. J. (2012). Transcriptome analysis of a long-lived natural Drosophila variant: a prominent role of stress-and reproduction-genes in lifespan extension. BMC genomics, 13(1), 167.

- Dussutour, A., Latty, T., Beekman, M., \& Simpson, S. J. (2010). Amoeboid organism solves complex nutritional challenges. Proceedings of the National Academy of Sciences, 107(10), 4607-4611.

- Emran, S., Yang, M., He, X., Zandveld, J., \& Piper, M. D. (2014). Target of rapamycin signalling mediates the lifespan-extending effects of dietary restriction by essential amino acid alteration. Aging (Albany NY), 6(5), 390-398.

- $\quad$ Esser, K. (1974). Podospora anserina. In Bacteria, Bacteriophages, and Fungi (pp. 531-551). Springer US.974

- $\quad$ Fabrizio, P., Pozza, F., Pletcher, S. D., Gendron, C. M., \& Longo, V. D. (2001). Regulation of longevity and stress resistance by Sch9 in yeast. Science, 292(5515), 288-290.

- Fanson, B. G., Fanson, K. V., \& Taylor, P. W. (2012). Cost of reproduction in the Queensland fruit fly: Y-model versus lethal protein hypothesis. Proceedings of the Royal Society of London B: Biological Sciences, rspb20122033.

- $\quad$ Farhadian, S. F., Suárez-Fariñas, M., Cho, C. E., Pellegrino, M., \& Vosshall, L. B. (2012). Post-fasting olfactory, transcriptional, and feeding responses in Drosophila. Physiology \& behavior, 105(2), 544-553.

- $\quad$ Flachsbart, F., Caliebe, A., Kleindorp, R., Blanché, H., von Eller-Eberstein, H., Nikolaus, S., ... \& Nebel, A. (2009). Association of FOXO3A variation with human longevity confirmed in German centenarians. Proceedings of the National Academy of Sciences, 106(8), 2700-2705.

- $\quad$ Flatt, T., Tu, M. P., \& Tatar, M. (2005). Hormonal pleiotropy and the juvenile hormone regulation of Drosophila development and life history. Bioessays, 27(10), 999-1010.

- Flatt, T., \& Kawecki, T. J. (2007). JUVENILE HORMONE AS A REGULATOR OF THE TRADE-OFF BETWEEN REPRODUCTION AND LIFE SPAN IN DROSOPHILA MELANOGASTER. Evolution, 61(8), 1980-1991.

- Flatt, T. (2011). Survival costs of reproduction in Drosophila. Experimental gerontology, 46(5), 369-375.

- $\quad$ Flatt, T., \& Heyland, A. (Eds.). (2011). Mechanisms of life history evolution: the genetics and physiology of life history traits and trade-offs. OUP Oxford.

- Flatt, T. (2014). Plasticity of lifespan: a reaction norm perspective. Proceedings of the Nutrition Society, 73(04), 532-542.

- Flyatlas: Chintapalli, V. R., Wang, J., \& Dow, J. A. (2007). Using FlyAtlas to identify better Drosophila melanogaster models of human disease. Nature genetics, 39(6), 715-720.

- Flybase: Gramates, L. Sian, et al. "FlyBase at 25: looking to the future." Nucleic acids research 45.D1 (2017): D663-D671.

- Fontana, L., Partridge, L., \& Longo, V. D. (2010). Extending healthy life span-from yeast to humans. science, 328(5976), 321-326.

- Fontana, L., \& Partridge, L. (2015). Promoting health and longevity through diet: from model organisms to humans. Cell, 161(1), 106-118.

- Friedman, D. B., \& Johnson, T. E. (1988). A mutation in the age-1 gene in Caenorhabditis elegans lengthens life and reduces hermaphrodite fertility. Genetics, $118(1)$, 75-86.

- $\quad$ Partridge, L., \& Gems, D. (2002). Mechanisms of aging: public or private?. Nature Reviews Genetics, 3(3), 165175. 
- Gentleman, R., Carey, V., Huber, W., Irizarry, R., \& Dudoit, S. (Eds.). (2006). Bioinformatics and computational biology solutions using R and Bioconductor. Springer Science \& Business Media.

- Gershman, B., Puig, O., Hang, L., Peitzsch, R. M., Tatar, M., \& Garofalo, R. S. (2007). High-resolution dynamics of the transcriptional response to nutrition in Drosophila: a key role for dFOXO. Physiological genomics, 29(1), 24-34.

- Ghalambor, C. K., McKay, J. K., Carroll, S. P., \& Reznick, D. N. (2007). Adaptive versus non-adaptive phenotypic plasticity and the potential for contemporary adaptation in new environments. Functional ecology, 21(3), 394-407.

- Goberdhan, D. C., Meredith, D., Boyd, C. R., \& Wilson, C. (2005). PAT-related amino acid transporters regulate growth via a novel mechanism that does not require bulk transport of amino acids. Development, 132(10), 23652375.

- Good, T. P., \& Tatar, M. (2001). Age-specific mortality and reproduction respond to adult dietary restriction in Drosophila melanogaster. Journal of insect physiology, 47(12), 1467-1473.

- Gordon, M. D., Ayres, J. S., Schneider, D. S., \& Nusse, R. (2008). Pathogenesis of listeria-infected Drosophila wntD mutants is associated with elevated levels of the novel immunity gene edin. PLoS Pathog, 4(7), e1000111.

- Grandison, R. C., Piper, M. D., \& Partridge, L. (2009). Amino-acid imbalance explains extension of lifespan by dietary restriction in Drosophila. Nature, 462(7276), 1061-1064.

- Greer, E. L., \& Brunet, A. (2009). Different dietary restriction regimens extend lifespan by both independent and overlapping genetic pathways in C. elegans. Aging cell, 8(2), 113-127.

- Grönke, S., Clarke, D. F., Broughton, S., Andrews, T. D., \& Partridge, L. (2010). Molecular evolution and functional characterization of Drosophila insulin-like peptides. PLoS genetics, 6(2), e1000857.

- Guarente, L. (2008). Mitochondria-a nexus for aging, calorie restriction, and sirtuins?. Cell, 132(2), $171-176$.

- Hahne, F., Huber, W., Gentleman, R., \& Falcon, S. (2010). Bioconductor case studies. Springer Science \& Business Media.

- Han, E. S., \& Hickey, M. (2005). Microarray evaluation of dietary restriction. The Journal of nutrition, 135(6), 1343-1346.

- Harper, J. M., Leathers, C. W., \& Austad, S. N. (2006). Does caloric restriction extend life in wild mice?. Aging cell, 5(6), 441-449.

- Harrison, D. E., \& Archer, J. R. (1988). Natural selection for extended longevity from food restriction.

- Heilbronn, L. K., de Jonge, L., Frisard, M. I., DeLany, J. P., Larson-Meyer, D. E., Rood, J., ... \& Greenway, F. L. (2006). Effect of 6-month calorie restriction on biomarkers of longevity, metabolic adaptation, and oxidative stress in overweight individuals: a randomized controlled trial. Jama, 295(13), 1539-1548.

- Henderson, S. T., Bonafè, M., \& Johnson, T. E. (2006). daf-16 protects the nematode Caenorhabditis elegans during food deprivation. The Journals of Gerontology Series A: Biological Sciences and Medical Sciences, 61(5), 444-460.

- Heuvel, J. V. D. (2014). The evolution and plasticity of life histories upon variation in nutrition: on aging focused integrative approach. Instituut Biologie Leiden (IBL), Faculty of Science, Leiden University.

- Heuvel, J., Zandveld, J., Mulder, M., Brakefield, P. M., Kirkwood, T. B. L., Shanley, D. P., \& Zwaan, B. J. (2014). The plastic fly: the effect of sustained fluctuations in adult food supply on life-history traits. Journal of evolutionary biology, 27(11), 2322-2333.

- Holliday, R. (1989). Food, reproduction and Longevity: Is the extended lifespan of calorie-restricted animals an evolutionary adaptation?. Bioessays, 10(4), 125-127.

- Holzenberger, M., Dupont, J., Ducos, B., Leneuve, P., Géloën, A., Even, P. C., ... \& Le Bouc, Y. (2003). IGF-1 receptor regulates lifespan and resistance to oxidative stress in mice. Nature, 421(6919), 182-187.

- Hou, L., Wang, D., Chen, D., Liu, Y., Zhang, Y., Cheng, H., ... \& Han, J. D. J. (2016). A Systems Approach to Reverse Engineer Lifespan Extension by Dietary Restriction. Cell metabolism, 23(3), 529-540.

- Huang, D. W., Sherman, B. T., \& Lempicki, R. A. (2008). Bioinformatics enrichment tools: paths toward the comprehensive functional analysis of large gene lists. Nucleic acids research, 37(1), 1-13. 
- Huang, C., Xiong, C., \& Kornfeld, K. (2004). Measurements of age-related changes of physiological processes that predict lifespan of Caenorhabditis elegans. Proceedings of the National Academy of Sciences of the United States of America, 101(21), 8084-8089.

- Ingram, D. K., Weindruch, R., Spangler, E. L., Freeman, J. R., \& Walford, R. L. (1987). Dietary restriction benefits learning and motor performance of aged mice. Journal of gerontology, 42(1), 78-81.

- Jensen, K., McClure, C., Priest, N. K., \& Hunt, J. (2015). Sex-specific effects of protein and carbohydrate intake on reproduction but not lifespan in Drosophila melanogaster. Aging cell, 14(4), 605-615.

- Jia, K., Chen, D., \& Riddle, D. L. (2004). The TOR pathway interacts with the insulin signaling pathway to regulate C. elegans larval development, metabolism and life span. Development, 131(16), 3897-3906.

- Jiang, J. C., Jaruga, E., Repnevskaya, M. V., \& Jazwinski, S. M. (2000). An intervention resembling caloric restriction prolongs life span and retards aging in yeast. The FASEB Journal, 14(14), 2135-2137.

- Johnson, T. E. (1990). Increased life-span of age-1 mutants in Caenorhabditis elegans and lower Gompertz rate of aging. Science, 249(4971), 908.

- Jones, O. R., Scheuerlein, A., Salguero-Gómez, R., Camarda, C. G., Schaible, R., Casper, B. B., ... \& QuintanaAscencio, P. F. (2014). Diversity of ageing across the tree of life. Nature, 505(7482), 169-173.

- $\quad$ Kaeberlein, M., Powers, R. W., Steffen, K. K., Westman, E. A., Hu, D., Dang, N., ... \& Kennedy, B. K. (2005). Regulation of yeast replicative life span by TOR and Sch9 in response to nutrients. Science, 310(5751), 11931196.

- $\quad$ Kafri, R., Springer, M., \& Pilpel, Y. (2009). Genetic redundancy: new tricks for old genes. Cell, 136(3), $389-392$.

- Kapahi, P., Zid, B. M., Harper, T., Koslover, D., Sapin, V., \& Benzer, S. (2004). Regulation of lifespan in Drosophila by modulation of genes in the TOR signaling pathway. Current Biology, 14(10), 885-890.

- Kayo, T., Allison, D. B., Weindruch, R., \& Prolla, T. A. (2001). Influences of aging and caloric restriction on the transcriptional profile of skeletal muscle from rhesus monkeys. Proceedings of the National Academy of Sciences, 98(9), 5093-5098.

- Kenyon, C., Chang, J., Gensch, E., Rudner, A., \& Tabtiang, R. (1993). A C. elegans mutant that lives twice as long as wild type. Nature, 366(6454), 461-464.

- Kirkwood, T. B. (1977). Evolution of ageing. Nature, 270(5635), 301-304.

- Kirkwood, T. B., \& Holliday, R. (1979). The evolution of ageing and longevity. Proceedings of the Royal Society of London B: Biological Sciences, 205(1161), 531-546.

- $\quad$ Kirkwood, T. B., \& Austad, S. N. (2000). Why do we age?. Nature, 408(6809), 233-238.

- Klass, M. R. (1977). Aging in the nematode Caenorhabditis elegans: major biological and environmental factors influencing life span. Mechanisms of ageing and development, 6, 413-429.

- Klass, M. R. (1983). A method for the isolation of longevity mutants in the nematode Caenorhabditis elegans and initial results. Mechanisms of ageing and development, 22(3), 279-286.

- Klepsatel, P., Gáliková, M., Huber, C. D., \& Flatt, T. (2014). Similarities and differences in altitudinal versus latitudinal variation for morphological traits in Drosophila melanogaster. Evolution, 68(5), 1385-1398.

- Knuppertz, L., Hamann, A., Pampaloni, F., Stelzer, E., \& Osiewacz, H. D. (2014). Identification of autophagy as a longevity-assurance mechanism in the aging model Podospora anserina. Autophagy, 10(5), 822-834.

- Kolesnikov, N., Hastings, E., Keays, M., Melnichuk, O., Tang, Y. A., Williams, E., ... \& Megy, K. (2014). ArrayExpress update-simplifying data submissions. Nucleic acids research, gku1057.

- $\quad$ Kristan, D. M. (2008). Calorie restriction and susceptibility to intact pathogens. Age, 30(2-3), 147.

- $\quad$ Lawler, D. F., Larson, B. T., Ballam, J. M., Smith, G. K., Biery, D. N., Evans, R. H., ... \& Kealy, R. D. (2008). Diet restriction and ageing in the dog: major observations over two decades. British Journal of Nutrition, 99(04), 793-805.

- Lee, C. K., Klopp, R. G., Weindruch, R., \& Prolla, T. A. (1999). Gene expression profile of aging and its retardation by caloric restriction. Science, 285(5432), 1390-1393.

- $\quad$ Lee, K. P., Simpson, S. J., Clissold, F. J., Brooks, R., Ballard, J. W. O., Taylor, P. W., ... \& Raubenheimer, D. (2008). Lifespan and reproduction in Drosophila: new insights from nutritional geometry. Proceedings of the National Academy of Sciences, 105(7), 2498-2503. 
- Libina, N., Berman, J. R., \& Kenyon, C. (2003). Tissue-specific activities of C. elegans DAF-16 in the regulation of lifespan. Cell, 115(4), 489-502.

- Liao, C. Y., Rikke, B. A., Johnson, T. E., Diaz, V., \& Nelson, J. F. (2010). Genetic variation in the murine lifespan response to dietary restriction: from life extension to life shortening. Aging cell, 9(1), 92-95.

- Lin, Y., Chen, Z. X., Oliver, B., \& Harbison, S. T. (2016). Microenvironmental Gene Expression Plasticity Among Individual Drosophila melanogaster. G3: Genes| Genomes| Genetics, 6(12), 4197-4210.

- Lorin, S., Dufour, E., \& Sainsard-Chanet, A. (2006). Mitochondrial metabolism and aging in the filamentous fungus Podospora anserina. Biochimica et Biophysica Acta (BBA)-Bioenergetics, 1757(5), 604-610.

- $\quad$ Luckinbill, L. S., Arking, R., Clare, M. J., Cirocco, W. C., \& Buck, S. A. (1984). Selection for delayed senescence in Drosophila melanogaster. Evolution, 996-1003.

- $\quad$ Maas, M. F., de Boer, H. J., Debets, A. J., \& Hoekstra, R. F. (2004). The mitochondrial plasmid pAL2-1 reduces calorie restriction mediated life span extension in the filamentous fungus Podospora anserina. Fungal Genetics and Biology, 41(9), 865-871.

- $\quad$ Maas, M. F. P. M., Sellem, C. H., Hoekstra, R. F., Debets, A. J. M., \& Sainsard-Chanet, A. (2007). Integration of a pAL2-1 homologous mitochondrial plasmid associated with life span extension in Podospora anserina. Fungal Genetics and Biology, 44(7), 659-671.

- Mackay, T. F. (2004). The genetic architecture of quantitative traits: lessons from Drosophila. Current opinion in genetics \& development, 14(3), 253-257.

- Magwere, T., Chapman, T., \& Partridge, L. (2004). Sex differences in the effect of dietary restriction on life span and mortality rates in female and male Drosophila melanogaster. The Journals of Gerontology Series A: Biological Sciences and Medical Sciences, 59(1), B3-B9.

- Mair, W., Goymer, P., Pletcher, S. D., \& Partridge, L. (2003). Demography of dietary restriction and death in Drosophila. Science, 301(5640), 1731-1733.

- Mair, W., Sgrò, C. M., Johnson, A. P., Chapman, T., \& Partridge, L. (2004). Lifespan extension by dietary restriction in female Drosophila melanogaster is not caused by a reduction in vitellogenesis or ovarian activity. Experimental gerontology, 39(7), 1011-1019.

- Mair, W., Piper, M. D., \& Partridge, L. (2005). Calories do not explain extension of life span by dietary restriction in Drosophila. PLoS Biol, 3(7), e223.

- Mair, W., \& Dillin, A. (2008). Aging and survival: the genetics of life span extension by dietary restriction. Annu. Rev. Biochem., 77, 727-754.

- Maklakov, A. A., Simpson, S. J., Zajitschek, F., Hall, M. D., Dessmann, J., Clissold, F., ... \& Brooks, R. C. (2008). Sex-specific fitness effects of nutrient intake on reproduction and lifespan. Current Biology, 18(14), 1062-1066.

- $\quad$ Mangel, M. (2001). Complex adaptive systems, aging and longevity. Journal of Theoretical Biology, 213(4), 559571.

- Maret, T. J., \& Collins, J. P. (1997). Ecological origin of morphological diversity: a study of alternative trophic phenotypes in larval salamanders. Evolution, 898-905.

- Marshall, K. E., \& Sinclair, B. J. (2009). Repeated stress exposure results in a survival-reproduction trade-off in Drosophila melanogaster. Proceedings of the Royal Society of London B: Biological Sciences, rspb20091807.

- $\quad$ Mattison, J. A., Roth, G. S., Beasley, T. M., Tilmont, E. M., Handy, A. M., Herbert, R. L., ... \& Barnard, D. (2012). Impact of caloric restriction on health and survival in rhesus monkeys from the NIA study. Nature, 489(7415), 318-321.

- May, C. M., Doroszuk, A., \& Zwaan, B. J. (2015). The effect of developmental nutrition on life span and fecundity depends on the adult reproductive environment in Drosophila melanogaster. Ecology and evolution, 5(6), 1156-1168.

- May, C. M. (2016). Linking growing up and getting old (Doctoral dissertation, Wageningen University).

- $\quad$ McCarroll, S. A., Murphy, C. T., Zou, S., Pletcher, S. D., Chin, C. S., Jan, Y. N., ... \& Li, H. (2004). Comparing genomic expression patterns across species identifies shared transcriptional profile in aging. Nature genetics, 36(2), 197-204. 
- $\quad$ McCay, C. M., Crowell, M. F., \& Maynard, L. A. (1935). The effect of retarded growth upon the length of life span and upon the ultimate body size one figure. The journal of Nutrition, 10(1), 63-79.

- Medawar, P. B. (1952). An unsolved problem of biology. College.

- Metaxakis, A., \& Partridge, L. (2013). Dietary restriction extends lifespan in wild-derived populations of Drosophila melanogaster. PloS one, 8(9), e74681.

- Min, K. J., Yamamoto, R., Buch, S., Pankratz, M., \& Tatar, M. (2008). Drosophila lifespan control by dietary restriction independent of insulin-like signaling. Aging cell, 7(2), 199-206.

- Minina, E. A., Sanchez-Vera, V., Moschou, P. N., Suarez, M. F., Sundberg, E., Weih, M., \& Bozhkov, P. V. (2013). Autophagy mediates caloric restriction-induced lifespan extension in Arabidopsis. Aging cell, 12(2), 327-329.

- Müller, I., Zimmermann, M., Becker, D., \& Flömer, M. (1980). Calendar life span versus budding lifespan of Saccharomyces cerevisiae. Mechanisms of ageing and development, 12(1), 47-52.

- Nakagawa, S., Lagisz, M., Hector, K. L., \& Spencer, H. G. (2012). Comparative and meta-analytic insights into life extension via dietary restriction. Aging cell, 11(3), 401-409.

- $\quad$ Okamoto, N., Yamanaka, N., Yagi, Y., Nishida, Y., Kataoka, H., O'Connor, M. B., \& Mizoguchi, A. (2009). A fat body-derived IGF-like peptide regulates postfeeding growth in Drosophila. Developmental cell, 17(6), 885-891.

- Oldham, S., Montagne, J., Radimerski, T., Thomas, G., \& Hafen, E. (2000). Genetic and biochemical characterization of dTOR, the Drosophila homolog of the target of rapamycin. Genes \& development, 14(21), 2689-2694.

- Oldham, S., \& Hafen, E. (2003). Insulin/IGF and target of rapamycin signaling: a TOR de force in growth control. Trends in cell biology, 13(2), 79-85.

- Osiewacz, H. D., Hermanns, J., Marcou, D., Triffi, M., \& Esser, K. (1989). Mitochondrial DNA rearrangements are correlated with a delayed amplification of the mobile intron (plDNA) in a long-lived mutant of Podospora anserina. Mutation Research/DNAging, 219(1), 9-15.

- Osiewacz, H. D. (2002). Genes, mitochondria and aging in filamentous fungi. Ageing research reviews, 1(3), 425-442.

- Osiewacz, H. D. (2011). Mitochondrial quality control in aging and lifespan control of the fungal aging model Podospora anserina.

- Overton, J. M., \& Williams, T. D. (2004). Behavioral and physiologic responses to caloric restriction in mice. Physiology \& behavior, 81(5), 749-754.

- Page, R. E., \& Peng, C. Y. S. (2001). Aging and development in social insects with emphasis on the honey bee, Apis mellifera L. Experimental gerontology, 36(4), 695-711.

- $\quad$ Partridge, L., Barrie, B., Barton, N. H., Fowler, K., \& French, V. (1995). Rapid laboratory evolution of adult lifehistory traits in Drosophila melanogaster in response to temperature. Evolution, 538-544.

- $\quad$ Partridge, L., Prowse, N., \& Pignatelli, P. (1999). Another set of responses and correlated responses to selection on age at reproduction in Drosophila melanogaster. Proceedings of the Royal Society of London B: Biological Sciences, 266(1416), 255-261.

- Partridge, L., \& Gems, D. (2002). Mechanisms of aging: public or private?. Nature Reviews Genetics, 3(3), 165175.

- Partridge, L., Alic, N., Bjedov, I., \& Piper, M. D. (2011). Ageing in Drosophila: the role of the insulin/Igf and TOR signalling network. Experimental gerontology, 46(5), 376-381.

- Pawlikowska, L., Hu, D., Huntsman, S., Sung, A., Chu, C., Chen, J., ... \& Psaty, B. M. (2009). Association of common genetic variation in the insulin/IGF1 signaling pathway with human longevity. Aging cell, 8(4), 460472.

- $\quad$ Perls, T. T., Alpert, L., \& Fretts, R. C. (1997). Middle-aged mothers live longer. Nature, 389(6647), 133-133.

- $\quad$ Pesch, Y. Y., Riedel, D., Patil, K. R., Loch, G., \& Behr, M. (2016). Chitinases and Imaginal disc growth factors organize the extracellular matrix formation at barrier tissues in insects. Scientific reports, 6 .

- Pfaffl, M. W. (2001). A new mathematical model for relative quantification in real-time RT-PCR. Nucleic acids research, 29(9), e45-e45. 
- Piersma, T., \& Drent, J. (2003). Phenotypic flexibility and the evolution of organismal design. Trends in Ecology \& Evolution, 18(5), 228-233.

- $\quad$ Piper, M. D., \& Partridge, L. (2007). Dietary restriction in Drosophila: delayed aging or experimental artefact?. PLoS Genet, 3(4), e57.

- $\quad$ Piper, M. D., Partridge, L., Raubenheimer, D., \& Simpson, S. J. (2011). Dietary restriction and aging: a unifying perspective. Cell metabolism, 14(2), 154-160.

- $\quad$ Piper, M. D., Blanc, E., Leitão-Gonçalves, R., Yang, M., He, X., Linford, N. J., ... \& Kerr, F. (2014). A holidic medium for Drosophila melanogaster. Nature methods, 11(1), 100-105.

- $\quad$ Piper M.D.W., Soultoukis G.A., Blanc E. ..., Simpson S.J, Ribeiro C., Partridge L. (2017), Matching Dietary Amino Acid Balance to the In Silico-Translated Exome Optimizes Growth and Reproduction without Cost to Lifespan. Cell Metabolism 25, 610-621

- Plank, M., Wuttke, D., van Dam, S., Clarke, S. A., \& de Magalhaes, J. P. (2012). A meta-analysis of caloric restriction gene expression profiles to infer common signatures and regulatory mechanisms. Molecular Biosystems, 8(4), 1339-1349.

- $\quad$ Pletcher, S. D., Macdonald, S. J., Marguerie, R., Certa, U., Stearns, S. C., Goldstein, D. B., \& Partridge, L. (2002). Genome-wide transcript profiles in aging and calorically restricted Drosophila melanogaster. Current Biology, 12(9), 712-723.

- Post, S., \& Tatar, M. (2016). Nutritional geometric profiles of insulin/IGF expression in Drosophila melanogaster. PloS one, 11(5), e0155628.

- Pradet-Balade, B., Boulmé, F., Beug, H., Müllner, E. W., \& Garcia-Sanz, J. A. (2001). Translation control: bridging the gap between genomics and proteomics?. Trends in biochemical sciences, 26(4), 225-229.

- $\quad$ Reed, M. J., Penn, P. E., Li, Y., Birnbaum, R., Vernon, R. B., Johnson, T. S., ... \& Wolf, N. S. (1996). Enhanced cell proliferation and biosynthesis mediate improved wound repair in refed, caloric-restricted mice. Mechanisms of ageing and development, 89(1), 21-43.

- Regan, J. C., Khericha, M., Dobson, A. J., Bolukbasi, E., Rattanavirotkul, N., \& Partridge, L. (2016). Sex difference in pathology of the ageing gut mediates the greater response of female lifespan to dietary restriction. Elife, 5, e10956.

- Roff, D. A., \& Fairbairn, D. J. (2001). The genetic basis of dispersal and migration, and its consequences for the evolution of correlated traits. Dispersal. Oxford University Press, Oxford, 191-202.

- $\quad$ Romero-Calderón, R., Shome, R. M., Simon, A. F., Daniels, R. W., DiAntonio, A., \& Krantz, D. E. (2007). A screen for neurotransmitter transporters expressed in the visual system of Drosophila melanogaster identifies three novel genes. Developmental neurobiology, 67(5), 550-569.

- $\quad$ Rose, M., \& Charlesworth, B. (1980). A test of evolutionary theories of senescence. Nature, 287(5778), $141-142$.

- Rose, M. R. (1984). Laboratory evolution of postponed senescence in Drosophila melanogaster. Evolution, 1004-1010.

- $\quad$ Rose, M. R., Vu, L. N., Park, S. U., \& Graves, J. L. (1992). Selection on stress resistance increases longevity in Drosophila melanogaster. Experimental gerontology, 27(2), 241-250.

- Rulifson, E. J., Kim, S. K., \& Nusse, R. (2002). Ablation of insulin-producing neurons in flies: growth and diabetic phenotypes. Science, 296(5570), 1118-1120.

- Sarov-Blat, L., So, W. V., Liu, L., \& Rosbash, M. (2000). The Drosophila takeout gene is a novel molecular link between circadian rhythms and feeding behavior. Cell, 101(6), 647-656.

- Schleifer, K. H., \& Kandler, O. (1972). Peptidoglycan types of bacterial cell walls and their taxonomic implications. Bacteriological reviews, 36(4), 407.

- Schleit, J., Wasko, B. M., \& Kaeberlein, M. (2012). Yeast as a model to understand the interaction between genotype and the response to calorie restriction. FEBS letters, 586(18), 2868-2873.

- $\quad$ Schleit, J., Johnson, S. C., Bennett, C. F., Simko, M., Trongtham, N., Castanza, A., ... \& Sutphin, G. L. (2013). Molecular mechanisms underlying genotype-dependent responses to dietary restriction. Aging cell, 12(6), 10501061. 
- Selman, C., Tullet, J. M., Wieser, D., Irvine, E., Lingard, S. J., Choudhury, A. I., ... \& Woods, A. (2009). Ribosomal protein S6 kinase 1 signaling regulates mammalian life span. Science, 326(5949), 140-144.

- Selman, C., Partridge, L., \& Withers, D. J. (2011). Replication of extended lifespan phenotype in mice with deletion of insulin receptor substrate 1 . PloS one, 6(1), e16144.

- $\quad$ Service, P. M., Hutchinson, E. W., MacKinley, M. D., \& Rose, M. R. (1985). Resistance to environmental stress in Drosophila melanogaster selected for postponed senescence. Physiological zoology, 58(4), 380-389.

- $\quad$ Sevier, C. S., \& Kaiser, C. A. (2002). Formation and transfer of disulphide bonds in living cells. Nature reviews Molecular cell biology, 3(11), 836-847.

- Shanley, D. P., \& Kirkwood, T. B. (2000). Calorie restriction and aging: a life-history analysis. Evolution, 54(3), 740-750.

- $\quad$ Sigrist, S. J., \& Lehner, C. F. (1997). Drosophila fizzy-related down-regulates mitotic cyclins and is required for cell proliferation arrest and entry into endocycles. Cell, 90(4), 671-681.

- Simpson, S. J., \& Raubenheimer, D. (1993). A multi-level analysis of feeding behaviour: the geometry of nutritional decisions. Philosophical Transactions of the Royal Society B: Biological Sciences, 342(1302), 381402.

- Simpson, S. J., \& Raubenheimer, D. (2007). Caloric restriction and aging revisited: the need for a geometric analysis of the nutritional bases of aging. The Journals of Gerontology Series A: Biological Sciences and Medical Sciences, 62(7), 707-713.

- Simpson, S. J., \& Raubenheimer, D. (2012). The nature of nutrition: a unifying framework from animal adaptation to human obesity. Princeton University Press.

- Skorupa, D. A., Dervisefendic, A., Zwiener, J., \& Pletcher, S. D. (2008). Dietary composition specifies consumption, obesity, and lifespan in Drosophila melanogaster. Aging cell, 7(4), 478-490.

- Slack, C., Giannakou, M. E., Foley, A., Goss, M., \& Partridge, L. (2011). dFOXO-independent effects of reduced insulin-like signaling in Drosophila. Aging cell, 10(5), 735-748.

- Slaidina, M., Delanoue, R., Gronke, S., Partridge, L., \& Léopold, P. (2009). A Drosophila insulin-like peptide promotes growth during nonfeeding states. Developmental cell, 17(6), 874-884.

- Soerensen, M., Dato, S., Christensen, K., McGue, M., Stevnsner, T., Bohr, V. A., \& Christiansen, L. (2010). Replication of an association of variation in the FOXO3A gene with human longevity using both case-control and longitudinal data. Aging cell, 9(6), 1010-1017.

- Solon-Biet, S. M., McMahon, A. C., Ballard, J. W. O., Ruohonen, K., Wu, L. E., Cogger, V. C., ... \& Gokarn, R. (2014). The ratio of macronutrients, not caloric intake, dictates cardiometabolic health, aging, and longevity in ad libitum-fed mice. Cell metabolism, 19(3), 418-430.

- Soultoukis, G. (2016). Exome-matching of dietary amino acids in flies and mice (Doctoral dissertation, Universität zu Köln).

- Stastna, J. J., Snoek, L. B., Kammenga, J. E., \& Harvey, S. C. (2015). Genotype-dependent lifespan effects in peptone deprived Caenorhabditis elegans. Scientific reports, 5.

- Stearns, S. C. (1992). The evolution of life histories (Vol. 249). Oxford: Oxford University Press.

- Stearns, S. C., Ackermann, M., Doebeli, M., \& Kaiser, M. (2000). Experimental evolution of aging, growth, and reproduction in fruitflies. Proceedings of the National Academy of Sciences, 97(7), 3309-3313.

- Sutphin, G. L., \& Kaeberlein, M. (2008). Dietary restriction by bacterial deprivation increases life span in wildderived nematodes. Experimental gerontology, 43(3), 130-135.

- Tatar, M., Kopelman, A., Epstein, D., Tu, M. P., Yin, C. M., \& Garofalo, R. S. (2001). A mutant Drosophila insulin receptor homolog that extends life-span and impairs neuroendocrine function. Science, 292(5514), $107-$ 110 .

- Tatar, M., Bartke, A., \& Antebi, A. (2003). The endocrine regulation of aging by insulin-like signals. Science, 299(5611), 1346-1351.

- Terzibasi, E., Lefrançois, C., Domenici, P., Hartmann, N., Graf, M., \& Cellerino, A. (2009). Effects of dietary restriction on mortality and age-related phenotypes in the short-lived fish Nothobranchius furzeri. Aging cell, $8(2), 88-99$. 
- $\quad$ van de Rest, O., Schutte, B. A., Deelen, J., Stassen, S. A., van den Akker, E. B., van Heemst, D., \& DibbetsSchneider, P. (2016). Metabolic effects of a 13-weeks lifestyle intervention in older adults: The Growing Old Together Study. Aging (Albany NY), 8(1), 111.

- Van den Heuvel, J., Zandveld, J., Mulder, M., Brakefield, P. M., Kirkwood, T. B. L., Shanley, D. P., \& Zwaan, B. J. (2014). The plastic fly: the effect of sustained fluctuations in adult food supply on life-history traits. Journal of evolutionary biology, 27(11), 2322-2333.

- Van den Heuvel, J. (2014). The evolution and plasticity of life histories upon variation in nutrition: on aging focused integrative approach. Instituut Biologie Leiden (IBL), Faculty of Science, Leiden University.

- $\quad$ van den Heuvel, J., Zandveld, J., Brakefield, P. M., Kirkwood, T. B., Shanley, D. P., \& Zwaan, B. J. (2017). Growing more positive with age: The relationship between reproduction and survival in aging flies. Experimental gerontology, 90, 34-42.

- $\quad$ van Diepeningen, A. D., Debets, A. J., Slakhorst, S. M., \& Hoekstra, R. F. (2008). Mitochondrial pAL2-1 plasmid homologs are senescence factors in Podospora anserina independent of intrinsic senescence. Biotechnology journal, 3(6), 791-802.

- $\quad$ van Diepeningen, A. D., Engelmoer, D. J., Sellem, C. H., Huberts, D. H., Slakhorst, S. M., Sainsard-Chanet, A., ... \& Debets, A. J. (2014). Does autophagy mediate age-dependent effect of dietary restriction responses in the filamentous fungus Podospora anserina?. Phil. Trans. R. Soc. B, 369(1646), 20130447.

- $\quad$ van Diepeningen, A. D., Maas, M. F., Huberts, D. H., Goedbloed, D. J., Engelmoer, D. J., Slakhorst, S. M., ... \& Sainsard-Chanet, A. (2010a). Calorie restriction causes healthy life span extension in the filamentous fungus Podospora anserina. Mechanisms of ageing and development, 131(1), 60-68.

- $\quad$ van Diepeningen, A. D., Slakhorst, S. M., Koopmanschap, A. B., Ikink, G. J., Debets, A. J., \& Hoekstra, R. F. (2010b). Calorie restriction in the filamentous fungus Podospora anserina. Experimental gerontology, 45(7), 516-524.

- Wei, M., Fabrizio, P., Hu, J., Ge, H., Cheng, C., Li, L., \& Longo, V. D. (2008). Life span extension by calorie restriction depends on Rim15 and transcription factors downstream of Ras/PKA, Tor, and Sch9. PLoS genetics, 4(1), e13.

- Weindruch, R., Walford, R. L., Fligiel, S., \& Guthrie, D. (1986). The retardation of aging in mice by dietary restriction: longevity, cancer, immunity and lifetime energy intake. J Nutr, 116(4), 641-54.

- Weindruch, R., \& Walford, R. L. (1988). Retardation of aging and disease by dietary restriction. CC Thomas.

- Weraarchakul, N., Strong, R., Wood, W. G., \& Richardson, A. (1989). The effect of aging and dietary restriction on DNA repair. Experimental cell research, 181(1), 197-204.

- West-Eberhard, M. J. (2003). Developmental plasticity and evolution. Oxford University Press.

- Westendorp, R. G., \& Kirkwood, T. B. (1998). Human longevity at the cost of reproductive success. Nature, 396(6713), 743-746.

- Whitaker, R., Gil, M. P., Ding, F., Tatar, M., Helfand, S. L., \& Neretti, N. (2014). Dietary switch reveals fast coordinated gene expression changes in Drosophila melanogaster. Aging (Albany NY), 6(5), 355-368.

- Willcox, B. J., Donlon, T. A., He, Q., Chen, R., Grove, J. S., Yano, K., ... \& Curb, J. D. (2008). FOXO3A genotype is strongly associated with human longevity. Proceedings of the National Academy of Sciences, 105(37), 1398713992.

- Williams, P. D., \& Day, T. (2003). Antagonistic pleiotropy, mortality source interactions, and the evolutionary theory of senescence. Evolution, 57(7), 1478-1488.

- Wolkow, C. A., Kimura, K. D., Lee, M. S., \& Ruvkun, G. (2000). Regulation of C. elegans life-span by insulinlike signaling in the nervous system. Science, 290(5489), 147-150.

- Wong, R., Piper, M. D., Wertheim, B., \& Partridge, L. (2009). Quantification of food intake in Drosophila. PloS one, 4(6), e6063.

- Wu, J. \& Gentry, R.I. (2017). gcrma: Background Adjustment Using Sequence Information. R package version 2.48.0.

- Yamamoto, R., Bai, H., Dolezal, A. G., Amdam, G., \& Tatar, M. (2013). Juvenile hormone regulation of Drosophila aging. BMC biology, 11(1), 85. 
- $\quad$ Yu, B. P., Masoro, E. J., Murata, I., Bertrand, H. A., \& Lynd, F. T. (1982). Life Span Study of SPF Fischer 344 Male Rats Fed AdLibitum or Restricted Diets: Longevity, Growth, Lean Body Mass and Disease. Journal of Gerontology, 37(2), 130-141.

- Zajitschek, F., Zajitschek, S. R., Friberg, U., \& Maklakov, A. A. (2013). Interactive effects of sex, social environment, dietary restriction, and methionine on survival and reproduction in fruit flies. Age, 35(4), 11931204.

- Zajitschek, F., Zajitschek, S. R., Canton, C., Georgolopoulos, G., Friberg, U., \& Maklakov, A. A. (2016, February). Evolution under dietary restriction increases male reproductive performance without survival cost. In Proc. R. Soc. B (Vol. 283, No. 1825, p. 20152726). The Royal Society.

- Zandveld, J., van den Heuvel, J., Zwaan, B. J., \& Piper, M. D. (2017). Both overlapping and independent mechanisms determine how diet and insulin-ligand knockouts extend lifespan of Drosophila melanogaster. npj Aging and Mechanisms of Disease, 3(1), 1.

- Zid, B. M., Rogers, A. N., Katewa, S. D., Vargas, M. A., Kolipinski, M. C., Lu, T. A., ... \& Kapahi, P. (2009). 4EBP extends lifespan upon dietary restriction by enhancing mitochondrial activity in Drosophila. Cell, 139(1), 149-160.

- Zoncu, R., Efeyan, A., \& Sabatini, D. M. (2011). mTOR: from growth signal integration to cancer, diabetes and ageing. Nature reviews Molecular cell biology, 12(1), 21-35.

- Zuur, A. F., Ieno, E. N., Walker, N. J., Saveliev, A. A., \& Smith, G. M. (2009). Zero-truncated and zero-inflated models for count data. In Mixed effects models and extensions in ecology with R (pp. 261-293). Springer New York.

- Zwaan, B., Bijlsma, R., \& Hoekstra, R. F. (1995). Direct selection on life span in Drosophila melanogaster. Evolution, 649-659. 


\section{SUMMARY}

Dietary restriction (DR), a moderate reduction in nutrient intake, improves health or extends lifespan across many species. Moreover, recent insights have shown that also the effects of specific nutrients are of importance for the beneficial effects of DR rather than intake alone. However, we still lack much insight through what mechanisms the lifespan increase through diet changes is exactly mediated.

To further increase our understanding of the genetic mechanisms of nutrient-dependent lifespan, in Chapter 2, 3, 4, and 5 I employed different methods of genetic interventions (i.e. a genetic knockout, natural genetic variation and experimental evolution) using the model species Drosophila melanogaster and Podospora anserina. To test whether the genetic interventions affected the diet response, a broad range of diets was applied, thereby taking the recent insights of nutritional geometry into account. Furthermore, the response of the fly's whole-genome transcription to different dietary treatments were assessed in Chapter 6 and 7 to identify and potentially disentangle genetic mechanisms for lifespan from those for reproduction.

Chapter 2 addressed the effects of a triple knockout in the insulin-IGF signalling (IIS) pathway, namely for three genes encoding insulin-like peptides in Drosophila (dilp2-3,5). The mutant showed a strong elevation of lifespan that was irrespective of food type, but also a strong reduction of the female fly fecundity. In addition, this assay also revealed that the same knockout can yield different interpretations for its function in the fly's diet response, which was strongly dependent per diet dimension under consideration (i.e. varying yeast, sugar, or its ratio in the diet). This observation set the stage for other experimental chapters in this thesis, where a broad range of diets was applied to depict the exact genotypic effects that are involved in the lifespan response to diet. For example, in Chapter 2, interactive effects were observed between dilp2-3,5 knockout and the lifespan response to dietary sugar, but however, not for the yeast component of the diet.

In Chapter 3, for the same experimental diets, gene expression responses in dilp2-3,5 knockout flies were measured to describe the general dynamics on the pathway level. Interestingly, expression of the remaining fly head-expressed dilp, dilp6, was elevated on higher yeast levels upon dilp2-3,5 knockout. Therefore, compensatory mechanisms within IIS might still partly mediate the lifespan response to yeast.

In Chapter 4 the natural genetic variation for the response to DR was explored in wild-derived strains of the fungus Podospora anserina. By applying a broad range of glucose concentrations in a synthetic medium, we constructed reaction norms for 62 natural occurring strains and showed considerable natural variation in the shape of the reaction norms, including the glucose concentration at which lifespan increased and how steeply the fungus' lifespan responds to diet (the slope $S$ ). Furthermore, I identified a significant correlation between a strain's general lifespan and both parameters, suggesting that the lifespan response to diet partly acts through a mechanism involved in the fungus' lifespan determination under high nutrient, growth and reproduction permissive, conditions. On moderate glucose restriction levels we showed that a reduced reproduction was not always associated with lifespan extension, which indicates that decoupling of these traits (that often trade-off) can be achieved.

An evolutionary perspective on diet response and the connection between reproduction and lifespan, two often interconnected traits in lifespan research, was provided in Chapter 5. Here, experimental evolution (EE) was performed in Drosophila melanogaster to test whether improved 
reproductive capacity (i.e. local adaptation) to three nutritionally distinct diets directly affected the lifespan response. Adaptation to the distinct nutritional conditions, had no consistent effect on the lifespan response to diet. Other life-history traits that I assessed could more consistently be associated with the evolutionary nutritional treatments, which together suggested that the adaptive genetic mechanisms increasing the fly's reproduction were not necessarily interconnected singly with a change of lifespan, but rather with a change in the whole life-history strategy.

By exploring the fly's whole-genome transcription response in a continuously changing environment, Chapter 6 continued on the evolutionary relevance of lifespan responses to diet. This type of fluctuations may better reflect the fly's natural ecological setting than the continuous diets typically applied in whole-genome transcription laboratory studies. This revealed that flies were able to respond quickly to diet fluctuations throughout lifespan by drastically changing their transcription pattern and, moreover, my results indicated that a large part of the whole-genome transcription response could be attributed to the female fly's reproduction. Because I measured the response of multiple life-history traits to the fluctuating diet changes, I was able to decouple groups of genes associated with lifespan from those associated with reproduction. This is an important step in the direction of unravelling the genetic architecture that specifically mediates the lifespan response to diet, which can be especially useful in whole-genome transcription studies.

In Chapter 7, the consistencies between studies for their whole-genome transcription responses upon DR were investigated. This revealed large transcriptomic variations on different regulatory levels, i.e. the level of whole-genome transcription, most significant genes, and also gene ontology. To test whether the observed inconsistent whole-genome transcription responses were primarily a reflection of the fly's reproduction, such as observed in Chapter 6, a new cohort of flies was subjected to different regimes that resulted in very different age-dependent reproduction patterns. By assessing whole-genome transcription in this cohort at two time points, the gene expression changes reflected the age-dependent reproduction patterns observed, rather than the lifespan phenotypes. Similar to Chapter 6, this again highlighted the importance of measuring multiple life-history traits for associating whole-genome transcription responses to lifespan effects of dietary restriction.

In Chapter 8 the acquired insights across the experimental chapters were synthesized, discussing the importance of assessing a broad range of nutrients for the interpretation of any genotypic effect, and in addition discussing the value of measuring multiple life-history traits for genetic associations. In this chapter I also suggested directions for future research in Drosophila and Podospora that may be valuable for further unravelling and understanding the mechanisms of diet responses in other organisms, including in humans. 


\section{ACKNOWLEDGEMENTS}

Doing $\mathrm{PhD}$ is quite bizarre. I think there are very few jobs out there that have the same level of freedom I experienced during my $\mathrm{PhD}$. The interesting projects I could pursue and follow-up and the opportunity to be involved in teaching made my $\mathrm{PhD}$ an unforgettable experience. Bas, thank you so much for providing me the opportunity having this ultimate free $\mathrm{PhD}$ in your lab, it was truly a great period. You were also very helpful at different stages of my $\mathrm{PhD}$. For example with the grant applications, but of course also your comments and discussions about my thesis, publications, and of course at the very end, your feedback was extremely useful and of very much value during the development and finalization of all thesis chapters.

Joost, ik werkte al vanaf het begin van jouw $\mathrm{PhD}$ met veel plezier samen. Het is ontzettend leuk om tot in de kleinste details met je praten over life-history in fruitvliegen. Je was een belangrijk klankbord tijdens mijn PhD, altijd zeer kritisch, overtuigd en vol van nieuwe ideeën. Dankjewel daarvoor. En natuurlijk bedankt dat je mijn paranymph wil zijn.

The first project of my PhD at the UCL in London was extremely important in my development of ideas about ageing and diet. Matt, it was very interesting to learn about your ideas. As you may witness reading my thesis, the ideas developed in your co-authored first chapter play an important role in many of the other thesis chapters. Also thanks for your relaxed way of supervising and for your useful comments on the different versions of manuscripts I have been sending. Also all other people at the IHA I am grateful for sharing their useful ideas on fruit fly and worm ageing.

Back in the Genetics lab of Wageningen I was surrounded by so many nice people, which made my stay great ('Ik voel me hier als een vis in het water'). Wytske, mother of Genetics, you are so important for all the new (and old) people in the department, thank you so much for making me feel at home at the very start, but also for all the funny chats, your interest in my life and $\mathrm{PhD}$, and thanks for the important administrative work you have been doing. Eric, you are such a relaxed and open guy. I like your sense of humour and it was very pleasant that I could share every thought with you. I missed you when you were away, and I'm glad you're back, hopefully 4evah. Florien, we started our $\mathrm{PhD}$ in the same period so it was great to share our experiences, future ideas and many other (mostly) science-related topics throughout our PhD's. I miss you opposite of me, especially the evening talks when we both were staying till late. Let's soon go for a beer in Zurich! Another evening worker, Ramon, I'm glad there is another person in the lab who had the same (Spanish?) routine/rhythm. Also it was a lot of fun to be both paranymph of Florien. Tina, I found it nice there was another fly person around, it was enjoyable to discuss new ideas, supervise students and think of experiments with you. Also thanks for sharing your transcriptome dataset, a crucial one for the important insights we obtained in Chapter 7. Alex, you crazy fella. Thanks for all the funny absurdist moments. In your presence I never felt too embarrassed to make stupid jokes, superfkoesnie! Another fellow-absurdist, Frank, had jij gezegd dat ik een lul was...? Always in for a laugh and a tear, it's great you were around from my start till now. Jianhua, you are a funny person. You have a talent to make people easily happy, thanks for bringing this vibe to Wageningen! In the same line, I think I hardly saw any moment a sad Valeria, thanks for that beautiful smile! Claudio, it was nice getting to know you, as being the same-generation-GeneticsPhD-student it's good to see there's some (near) scientific future for us in the Netherlands... Cris, it's interesting to know a person who is so much into die-hard running, I've observed the plasticity of your calves with great interest, growing immensely the last 4 years. Lennart, the other extremesportsman, I expect at least a world championship before your defence... 
Marjon, you have a unique amount of energy and interest in other people. It was great to have you around last years of my PhD and I wish you all the best in Groningen. Santos, as I now call you Diego, I think you changed more into one of my colleagues than an (ex-)neighbour. It was fun to see you at work now. Mark, all the best at the NIOO, you inspired me to produce the song 'veni vidi vici' (freely available on Youtube), being the example that also on the long term a career in science is possible. Andy, it's my goal to go for a beer before I move to Amsterdam, the $11^{\text {th }}$ of October I'll be in Wageningen... Lidia, thanks for transferring the flies so often for me and your interest in the fly work. I hope will can still continue the interesting experiments you started. Gabriella, also thanks a lot for the fly maintenance and making me aware to keep the fly room and the cooking room tidy. Marijke, thanks for showing and sharing your Podospora skills, and for your help in setting up course practicals. Many thanks to you too Bertha, you have been very helpful in the fly RNA extractions and the fly transfers.

Many work and insights I got from student projects. Tom, Berdien, Dennis, Remco and Suzette. All of your projects gave me more and very useful insights for my own PhD. Remco, ontzettend bedankt voor het ongelooflijk veel voedsel maken in een sneltreinvaart, hierdoor kon ik voor al mijn evolutielijnen tegelijk de levensduur meten. Zonder jouw hulp was hoofdstuk 6 veel minder goed van de grond gekomen. Suzette, hoofdstuk 4 had voor mij de werknaam 'PodSuzette', bedankt voor het creëren van zo'n mooie dataset. Ik hoop dat we in de (nabije) toekomst kunnen blijven samenwerken. Jij bent op en top geschikt voor een $\mathrm{PhD}$ en ik zal met heel veel plezier betrokken zijn bij je projecten! Erik van den Akker, bedankt voor de hulp bij de transcriptieanalyses die uiteindelijk hebben geleid tot hoofdstuk 7 .

All people involved in TGAC during my $\mathrm{PhD}$ : Sijmen, Frank, Evelien, Florien, Wytske, Anneloes, Margot, Sabine, Kim, Ramon, (Mathijs, Setareh, Sara), it was nice to think about activities and organise events together. Also in preparation of a defence performance or Christmas dinner there was always a big group of enthusiast people to join The Awesome Genetics Choir (TAGC), thanks for all the fun while singing. All other people that were or are still around in the lab who really helped maintaining a good atmosphere: Klaas, Robert, Erik, Roxanne, Vanesa, Corrie, Padriac, Roel, Tom, René, Bernard, Justin, Kriti.

Bas, Fons, Duur, Lars and Arjan, thanks for giving me the opportunity to have so much involvement in the courses you (co-)coordinate. I think the experimental evolution of drawings could not have been developed so easily without implementing it in your courses. With a lot of pleasure I was involved in the courses. Fons, co-promotor, helaas is mijn $\mathrm{PhD}$ toch wat meer richting werk aan Drosophila gegaan dan we van te voren misschien beoogden. Ik ben nog steeds van mening dat Podospora een geweldig systeem is, en heb genoeg ideeën (nu wel) voor de toekomst. Eén ervan wordt op dit moment van schrijven tot uitvoering gebracht door studenten van de healthy ageing cursus... Bart, het was leuk een ander insectenpersoon erbij te hebben. Het laatste jaar hebben we meer met elkaar gewerkt. Ik vond het echt superleuk en interessant om samen een (enorm) symposium op ESEB te organiseren, supernuttig en het zou leuk zijn als er een mooie review uit voortkomt.

But there were more Radix inhabitants that brought some distraction and relaxation during my working days... Alexandre, Otto, Samira, Kris, it was fun to play together at the Friday Radix jam sessions and at the Bongerd. I still listen sometimes to recordings of our hit 'poe-poe-poe-poe, heeeee'. Matilda, Robert, Herman, Wim, Roos, Kris allemaal dank voor de ontspannende wandelingen of gezellige koffie-momenten in het Radix. 
When I left the city of my heart (Leiden) to live and work in Wageningen I had my doubts whether I could feel at home here. For me, people have always been an important factor determining my happiness and during my PhD period I was surrounded by a bunch of fantastic people. Living in Droevendaal was an unique experience, it was funny, free, sometimes restless, but overall very gezellig en warm to have lived there. Droef 47, you truly felt like a family and I keep great memories of this important period during my stay in Wageningen. I'm mainly thinking of the cosy "family dinners", but also the numerous breakfast parties (e.g. the day of the pigeon lovers) or the 'Lekker Dansen in de Tuin' party. Roeland, Evelien, Veronica, Johan, Merel, Lukas, Michael, Giulia, Thibault, Monica, Lalo, Jan-Dirk, Anna, Bosvos, Gerard and Henk, thank you all, it was great!

Roeland, jij was de perfecte persoon om alle loze gedachten en gevoel voor humor en absurditeit mee te delen. Ik heb echt zo enorm veel met je beleefd en enorm veel samen gelachen, teveel om op te noemen, van de ellenlange opgenomen sessies van 'Doet dat een keer', Paradise walk de musical, tot de ka-BAM video's in Lyon. Het was fijn om een dag te beginnen en hele ochtenden muziek te maken, lekker koffie te drinken en loos te kletsen. Fijn dat je mijn paranymph wilt zijn!

De Rozen, jullie zijn zulke leuke mensen, en ben blij dat ik jullie ken! Roos, bij elke oefening of brandalarm van het Radix denk ik aan je, onze gemeenschappelijke "liefde" voor het Radix schept een band. Je bent een mooi persoon en ik hoop je nog lang te blijven zien! Rooz, jammer dat je bent vertrokken naar je mooie Frankrijk, ik wordt altijd blij als ik je zie, vind je een grappig mens en supergezellig. Merel, jammer dat ik nu pas zo enthousiast wordt om met je te tennissen... Ik zal onze gezellige gesprekken missen. Mart en Mira, ik raakte al snel met jullie bevriend toen ik hier kwam wonen, en ik ben blij dat we ook na jullie vertrek nog steeds goed contact hebben, ik heb nog steeds zin in de sauna... Louise, bedankt voor de vele gezellige, fijne, rustige wandelingen door het (soms nachtelijke) Paradijs, deed me altijd goed!

De voetballers van KYB, Arjen, Bas, Dion, Duur, Joep, Marcel, Marco, Rob, Thomas, het was heerlijk om eens in de week helemaal kapot te gaan, en nadat iedereen was bekomen van de grote uitslag, een lekker biertje te drinken. Bedankt voor het blijven vragen naar wanneer ik nou eens klaar was...

Maaike, Jan (en later ook Lysanne), we hebben mooie avonden beleefd, vanaf ons eerstejaarsweekend, naar kerstfeest op het NIOO, PE\&RC afterparties in de Zaaier, tot op het laatstejaarsweekend, het werd altijd weer laat en gezellig. Dank jullie voor deze mooie avonden!

Van de (oude) Wageningen groep (Amber, Deirdre, Eva, Jos, Maarten, Maartje, Marije, Marije, Marco, Marlinde, Matilda, Meike, Pieter, Wouter) was bijna iedereen weer weggetrokken toen ik eindelijk in Wageningen woonde... Wouter, het was gezellig in den beginne van mijn $\mathrm{PhD}$ nog even samen met je in Wageningen gewoond te hebben. Nu kom ik weer dichterbij, dus heb wel zin in mooie nachtvlinderavonden. Marco en Matilda, dank voor het blijven, en iedereen voor de leuke weekenden met spelletjes, wandelingen of andere gezelligheid. De Zoetermeerse boys, het was gezellig weekendjes weg te gaan. Dank aan alle mooie Leidse Biologen, vooral de Tatra trip was natuurlijk onvergetelijk. Jammer dat ik steeds minder in Leiden ben geweest laatste jaren maar ik heb goede hoop, ik won straks weer dichter bij de stad van mijn hart! Maarten, bedankt voor het tellen van al die eitjes (inmiddels meer dan 6 jaar geleden), je ziet je werk terug in hoofdstuk 6 ! Daniel, het was lekker en gezellig fietsen van de zomer! Uri, ik vind het leuk dat je altijd zoveel interesse toont in de wetenschappelijke wereld. Je stelt leuke en ongebruikelijke vragen die me stimuleren op een andere manier naar wetenschap en evolutie te kijken. Ditzelfde geldt voor het 
samen telkens doorontwikkelen en geven van 'Creëer Je Eigen Evolutie!', dankje! Iteke, alias transSiberische Olga, het was echt een geweldige ervaring om de trans-Mongolië-express met je te bereizen! Deze heerlijke 5 weken break van mijn PhD deed me ontzettend goed. Ik denk nog vaak aan deze reis, en we moeten maar gauw de laatste week in de reisagenda bijwerken... Daniel en Joost, onze jaarlijkse evaluatieavondjes waren altijd gezellig en supermotiverend, dit gaf me altijd hernieuwde energie om even wat goeie wetenschappelijke stappen te zetten die een jaar later toch weer te ambitieus bleken.

Tom, Franske, Marthe en Edje, ik vind het altijd gezellig met jullie, ik heb er een nieuwe familie bijgekregen. Ik zie jullie altijd met veel plezier. Dank jullie wel dat jullie mij op zo'n natuurlijke manier deel te laten zijn van de familie!

Floor en Kris, het was zo ontzettend fijn om een nieuw huisje met jullie te betrekken, beter dan met jullie had ik het volgens mij niet kunnen treffen! Ik ben blij dat we ruim anderhalf jaar geleden dit zijn gestart. Jullie hebben beiden veel aandacht voor anderen en de natuur, zijn superrelaxt, sociaal en creatief en vormden daardoor aan de Ooststeeg een enorm goede basis voor een fijn leven, ik zal jullie gaan missen! Floor, ik heb genoten van je openheid, je gevoel voor humor, je gezelligheid en je enorme aandacht voor anderen. Kris, het was ontzettend fijn om met iemand te wonen die ook een $\mathrm{PhD}$ doet en net als ik enthousiast is over educatie. Het was geweldig als je bij ons thuis oefende met Andoorn en Nubia.

Pappa, Mamma, Maarten, Mira en Nele. Ik hou van jullie. Jullie zijn heel belangrijk voor me, ik voel me altijd thuis met en bij jullie en kan altijd alles kwijt, jullie zijn er altijd voor me, zeker in het begin van mijn $\mathrm{PhD}$ heb ik dit soms nodig gehad. Maart, laten we gauw weer broertjesweekend hebben! Pap, bedankt voor het maken van de voorkant, ik ben er erg blij mee!

Lieve Mathilde, ik ben zo ontzettend gelukkig met je en hou ontzettend veel van je! Ik vind je zo fijn, een goed mens en oprecht. Je geeft me energie en ik kom tot rust bij je, echt superbelangrijk, vooral tijdens de drukkere (laatste) periode van mijn PhD. Ik heb ontzettend veel zin om samen te gaan wonen in Amsterdam en nog meer van elkaars leven te gaan uitmaken en te delen! 


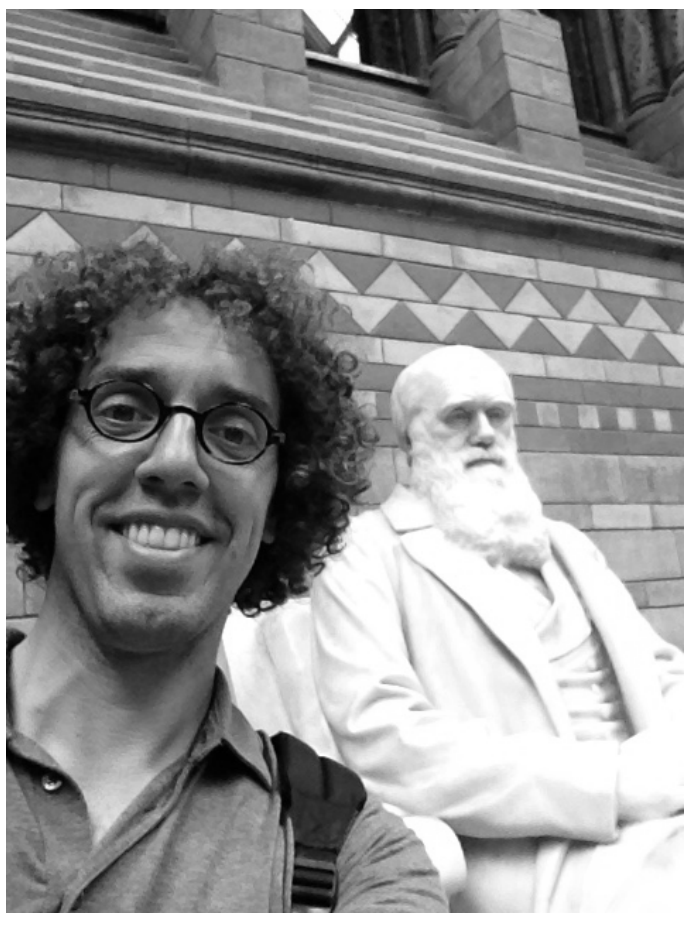




\section{BIOGRAPHY}

On July $21^{\text {st }} 1983$, Jelle Zandveld was born in the Antoniushove Hospital in Leidschendam as son of two loving parents, Bart and Thea Zandveld, and as a 3.5 years younger brother of Maarten Zandveld, an equally nice person. In the two following decades he experienced an enjoyable youth in Zoetermeer.

After the public primary school 'The Watersnip', he went to the 'Pallas College' and obtained a HAVO diploma in the year 2000. In the same year he began his first study, Technology of Human Movement (Bewegingstechnologie) at The Hague University of Applied Sciences. After five years he graduated on the subject of heavy weight training in endurance sports. The following year, he worked as a technical education assistant for Biology, Physics, Chemistry and other Beta courses at the Christian school community 'De Populier' in The Hague. This period provided the insight to start studying Biology at Leiden University. The time he studied Biology was an extremely interesting period in which he was very aware of what he wanted to learn. The nature trips with other Leiden biologists further opened his eyes to nature in general, and more specific to northern shovelers and moths. The nice residence in Leiden, a great study there, a pleasurable job at SportCity, and not the least the $2 / 3$ October festivals, opened a deep love for the city of Leiden.

During Jelle's study he was running projects with Dr. Joost van den Heuvel, which raised further interest in diet effects in fruit flies. For his MSc thesis Jelle continued studying fruit flies at the UCL in London in the lab with dr. Matthew Piper. The project on the role of 'Drosophila insulin-like peptides' in food-based aging deepened his interest in this area and made clear that he was determined to pursue a $\mathrm{PhD}$ position around this subject.

During his MSc, Jelle's Dutch supervisor, Dr. Bas Zwaan, was appointed in Wageningen as the new Professor of Genetics. At his department Jelle applied for a $\mathrm{PhD}$ position on the topic of genetics of food-related aging in the fruit fly Drosophila melanogaster and the ageing fungus Podospora anserina. In the first year of his $\mathrm{PhD}$, Jelle returned to the London lab to finalize the research he started during his MSc. The other years of his $\mathrm{PhD}$ Jelle spent in Wageningen, further investigating gene expression responses to diet, effects of natural variation and experimental evolution on food-related aging, but also analysed whole genome transcription in response to diet. During his PhD, Jelle organized symposia at the NERN and ESEB meeting, developed (together with visual artist Adam Uriel) the workshop 'Creëer Je Eigen Evolutie!' and was involved in different education courses. In 2016 he obtained a grant to further develop and implement an educational method to explain the conditions of evolution.

Before finishing his $\mathrm{PhD}$, Jelle already started working as a teacher at the Institute of Interdisciplinary Studies at the University of Amsterdam where he is involved in evolution education, but he is determined to continue working on running projects on ageing at the Genetics department in Wageningen. 


\section{LIST OF PUBLICATIONS}

Emran, S., Yang, M., He, X., Zandveld, J. and Piper, M.D., 2014. Target of rapamycin signalling mediates the lifespan-extending effects of dietary restriction by essential amino acid alteration. Aging (Albany NY), 6(5), p.390.

van den Heuvel, J., Zandveld, J., Mulder, M., Brakefield, P.M., Kirkwood, T.B.L., Shanley, D.P. and Zwaan, B.J., 2014. The plastic fly: the effect of sustained fluctuations in adult food supply on life-history traits. Journal of evolutionary biology, 27(11), pp.2322-2333.

Zandveld, J., van den Heuvel, J., Zwaan, B.J. and Piper, M.D., 2017. Both overlapping and independent mechanisms determine how diet and insulin-ligand knockouts extend lifespan of Drosophila melanogaster. npj Aging and Mechanisms of Disease, 3(1), p.1.

van den Heuvel, J., Zandveld, J., Brakefield, P.M., Kirkwood, T.B., Shanley, D.P. and Zwaan, B.J., 2017. Growing more positive with age: The relationship between reproduction and survival in aging flies. Experimental gerontology, 90, pp.34-42.

Zandveld, J., den Heuvel, J., Mulder, M., Brakefield, P.M., Kirkwood, T.B., Shanley, D.P. and Zwaan, B.J., 2017. Pervasive gene expression responses to a fluctuating diet in Drosophila melanogaster. Evolution. 


\section{PE\&RC Training and Education Statement}

With the training and education activities listed below the $\mathrm{PhD}$ candidate has complied with the requirements set by the C.T. de Wit Graduate School for Production Ecology and Resource Conservation (PE\&RC) which comprises of a minimum total of 32 ECTS (= 22 weeks of activities)

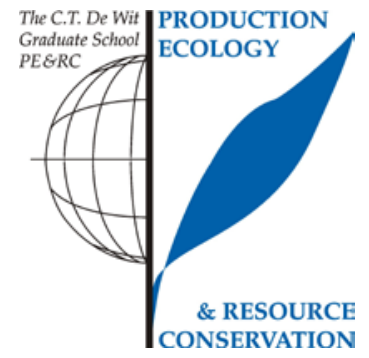

\section{Review of literature (4.5 ECTS)}

- Mechanisms of nutrient-dependent ageing in Podospora anserina and Drosophila melanogaster

\section{Writing of project proposal (4.5 ECTS)}

- Genetic mechanisms of nutrient-dependent ageing in Podospora anserina and Drosophila melanogaster

\section{Post-graduate courses (3 ECTS)}

- The power of RNA-seq; WGS (2016)

- EvoKE Workshops: the art of evolution (literature, art, theatre): using art to communicate evolution; evolution and public science literacy; attitudes and understanding of evolution; Porto, Portugal (2017)

\section{Laboratory training and working visits ( 3 ECTS)}

- The role of dilps in lifespan and DR response in Drosophila melanogaster; Institute of Healthy Ageing, UCL, UK (2012)

\section{Competence strengthening / skills courses ( 3 ECTS)}

- Techniques for writing and presenting a scientific paper; WGS (2012)

- $\quad$ Project and time management; WGS (2013)

- $\quad$ Ethics and philosophy in life sciences; WGS (2015)

PE\&RC Annual meetings, seminars and the PE\&RC weekend (2.4 ECTS)

- $\quad$ PE\&RC First years weekend (2012)

- $\quad$ PE\&RC Day (2012-2014)

- PE\&RC Last years weekend (2016)

\section{Discussion groups / local seminars / other scientific meetings (7 ECTS)}

- $\quad$ Evolution lunch meeting (2012-2016)

- Experimental evolution, Friday lunch meetings (2012-2016)

\section{International symposia, workshops and conferences (18.8 ECTS)}

- $\quad$ EMPSEB; Virrat, Finland (2012)

- NAEM; Lunteren, the Netherlands (2012-2015)

- ESEB; Lisbon, Portugal; ESEB; Lausanne, Swiss; ESEB; Groningen, the Netherlands $(2013,2015,2017)$

- $\quad$ DR Symposium, UK (2016)

\section{Lecturing / supervision of practicals / tutorials (27 ECTS)}

- MEE (2012-2013)

- Theories and concepts of healthy ageing (2013-2016)

- Insect ecology (2013-2016)

- $\quad$ Evolution \& systematics (2014-2017)

- $\quad$ GATC (2015-2017)

\section{Supervision of MSc students (6 ECTS)}

- Heritability of the DR response in Drosophila melanogaster

- Natural variation in the DR response of Podospora anserina 



\section{COLOFON}

The research of this thesis was carried out at the Laboratory of Genetics at Wageningen University and Research, Wageningen, the Netherlands and was financially supported by grants from the EU's 6th (Network of Excellence LifeSpan FP/036894) and 7th (IDEAL FP7/2007-2011/259679) Framework Programmes.

Cover design by: Bart Zandveld Thesis Layout by: Jelle Zandveld

Printed by: Proefschriftmaken 